\title{
Analysis and Modeling of Rutting for Long Life Asphalt Concrete Pavement
}

Berhanu Abesha Feyissa

Fachgebiet Straßenwesen

Technische Universität Darmstadt

Darmstadt 2009

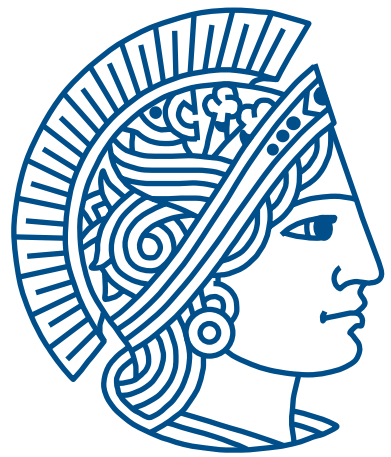

\section{TECHNISCHE UNIVERSITÄT DARMSTADT}




\section{Analysis and Modeling of Rutting for Long Life Asphalt Concrete Pavement}

By

Berhanu Abesha Feyissa

Department of Civil Engineering and Geodesy

Technische Universität Darmstadt 


\section{Analysis and Modeling of Rutting for Long Life Asphalt Concrete Pavement}

Department of Civil Engineering and Geodesy Technische Universität Darmstadt

Submitted by: Berhanu Abesha Feyissa

Supervisors: Prof. Dr. Ing. J. Stefan Bald

Prof. Dr. Ing. Michael Vormwald

Date of Submission: 01 September 2009

Date of Examination: 05 October 2009

Darmstadt 2009

D 17 
TECHNISCHE

UNIVERSITÄT

DARMSTADT

Fachbereich 13

Bauingenieurwesen und Geodäsie

Institute für Verkehr

Fachgebit Straßenwesen

\section{Analyse und Modellierung der Spurrinnenbiedung von Straßenbefestigung aus Asphaltbeton mit erhöhtes Lebensdauer}

Vom Fachbereich Bauingenieurwesen und Geodäsie der Technischen Universität Darmstadt zur Erlangung des akademischen Grades eines Doktor-Ingenieurs (Dr. Ing.) genehmigte Dissertation

Vorgelegt von

M.Tech. Berhanu Abesha Feyissa aus Bahir Dar, Äthiopien

D 17

Darmstadt 2009 


\section{Abstract}

Asphalt concrete (AC) pavement is one of the most important infrastructures that involve multiple layers of different materials subjected to non-uniform traffic loadings and varying environmental conditions. The repetitive traffic loadings that the road experiences during its service life, combined with temperature fluctuations, cause rutting, fatigue and other forms of deteriorations, which ultimately degrade the performance and durability of pavement structures. Both traffic volume and loads are increasing from year to year with rapid rate. The various categories of vehicles with different performance and operational characteristics contribute to pavement distresses. The dynamic load generated by heavy vehicles is considered as the primary cause of road structural damage and contribute the greater portion of the deterioration of AC pavements. Besides, the influence of temperature, which varies with time and along the pavement depth, makes an ever-changing temperature field. Extreme low daily temperature during winter and high temperature and solar radiation during summer cause thermal cracking and permanent deformation, respectively. The temperature gradient down to the depth has a direct influence on the properties of the AC structures, and hence, affects the response of a pavement while subjected to load.

Estimation and prediction of the maximum distresses likely to happen within the pavement layer is vital for proper design of the pavement. Constitutive modeling of the deformation behavior of AC enables to evaluate the deterioration progress. On the other hand, better understanding of materials behaviors and structural responses of asphalt concrete in combination with of loadstress-strain computation tools, allows for optimization of asphalt pavement thickness and material choice. Until recently, AC pavements have been designed to serve for about 20-25 years, however, it has been observed that some roads have been serving for more than 40 years without the need for structural rehabilitation. Such phenomenon has strengthened the issue of prolonged service life of road pavements to be remained as a key concern for road industries for the past several years.

In this research, the response of asphalt concrete has been evaluated by employing the ABAQUS finite element model. In order to take into account the effect of temperature on materials properties as well as to determine the deformation progress of a pavement under different temperature and loading conditions, a rut prediction model is developed. Various parameters critically influencing the accumulation of permanent deformation are evaluated by the Burgermaterial-based model developed by the Author. The FE analyses mainly have focused on the stress-strain state of AC pavement with varying loading conditions, structural geometry and material properties. The analysis showed keeping structural geometry, loading condition and other material parameters constant, an increase the E-moduli of the binder twice yields $4.9 \%$ and 14.3\% reduction in vertical stresses developed at the mid-depth of the binder and base layers, respectively and increasing the same by forth fold yields as well $11.1 \%$ and $27.6 \%$ in these layers. However, there is a slight increase in stress in the wearing layer, in both cases. This holds true as well in case of increasing the E-moduli of the base course, there are increase in stresses developed on both wearing and binder courses. The FE analyses show that critical shear stresses are developed within the binder layer. It has been observed that the magnitude of the vertical stress in the wearing course is independent of the wheel configuration, however, the overlapping of stresses is gradually pronounced in the binder course. The analyses reveal that the subbase 
and subgrade layers suffer greatly due to the influence of the overlapping of stresses developed by dual/multiple wheels. The vertical stress developed at the top layers of the asphalt concrete is one of the major contributors for rut formation on this layer. Of course the rate of rut formation is a function of the frequency of such load application, materials properties and other external factors such as temperature.

A Burger-based rutting model has been developed which can predict the accumulation of permanent deformation throughout the service life of a pavement with varying traffic load spectrum and annual and daily temperature variations. The model takes into account the hourly, daily and annual variations of the traffic, the expected traffic wheel loads and operating speed. At the same time, the time variation of temperature across the pavement depth are evaluated and associated with the material properties of the structure layers. It has been found that the higher the viscous component of the material the better the serviceability of the road without undergoing excessive deformation. Higher growth rate accelerates deformation. In addition, the structural geometries have an important contribution for the reduction of the over all permanent deformation of a pavement. It is recommended to work further on development of fatigue and other forms of damage prediction model under various loading spectrum and temperature ranges and integrate together for full scale analysis of AC pavements. 


\section{Zusammenfassung}

Straßen aus Asphaltbeton (AC) sind eine der wichtigsten Infrastrukturen. Sie bestehen aus mehreren Schichten unterschiedlicher Materialien. Sie sind ungleichförmigen Verkehrsbelastungen und unterschiedlicher Umgebungsbedingungen unterworfen. Die Verkehrsbelastungen, die die Straßen während ihrer Nutzungsdauer erleiden, führen zusammen mit dem ständigen Wechsel der Temperatur zu Spurrinnenbildung und Ermüdung, die schließlich die Benutzbarkeit und Haltbarkeit der Struktur massiv beeinträchtigen. Verkehrsaufkommen und Lasten erhöhen sich von Jahr zu Jahr immer schneller. Die verschiedenen Fahrzeugarten mit unterschiedlichen Eigenschaften erschweren Konstruktion und Analyse. Als Hauptursache für die Straßenschädigung wird die dynamische Belastung durch die schweren Fahrzeuge angesehen. Darüber hinaus führen die ständig wechselnden Temperaturen zu Einwirkungen, die über Zeit und die Tiefe variieren. Extreme niedrige tägliche Temperatur während des Winters verursachen thermische Risse, extrem hohe Temperaturen und Solarstrahlung während des Sommers verursachen dauerhafte Verformungen. Die Temperaturunterschiede über die Tiefe beeinflussen schließlich die Eigenschaften der Asphaltstraßenstrukturen, vor allem ihre Steifigkeit und Belastbarkeit.

Schätzung und Vorhersage der maximalen Belastungen, die eine Straßenschicht wahrscheinlich erleidet, ist wichtig für ihren angemessenen Entwurf. Die bewusste Modellierung des Verformungsverhaltens der Straße ermöglicht, den Zerstörungsprozess vorher zu sagen. Andererseits erlaubt ein besseres Verständnis des Materialverhaltens die Optimierung der Asphaltbetondicke und der Materialauswahl. Bis vor kurzem wurden Asphaltstraßen für eine Lebensdauer von 20-25 Jahren entworfen. Es wurden aber auch Straßen beobachtet, die mehr als 40 Jahre ohne die Notwendigkeit einer strukturellen Erneuerung benutzt werden konnten. Dieser Umstand verstärkt den Wunsch der Straßenbauer, die verlängerte Nutzungsdauer von Straßen auch zukünftig als Schlüsselfrage anzusehen.

In dieser Arbeit wird das FiniteElement (FE)-Programm ABAQUS benutzt, um das Verhalten des Asphaltbetons zu modellieren. Um den Effekt der Temperatur auf die Materialeigenschaften sowie die Verformungsentwicklung der Asphaltschichten unter verschiedenen Temperatur- und Lastbedingungen zu berücksichtigen, wird ein eigenes Spurrinnen-Vorhersagemodell entwickelt. Darin werden verschiedene Parameter, die die dauerhaften Verformung kritisch beeinflussen, mit Hilfe eines vom Autor entwickelten Burger-Material-Modells berücksichtigt. Die FE-Analysen werden dabei hauptsächlich verwendet, um den Spannung-Dehnungszustand des Oberbaus bei unterschiedlichen Lastzuständen, Abmessungen und Materialeigenschaften zu ermitteln. Die Analyse zeigt, dass bei sonst gleichen Abmessungen, Belastungen und Materialeigenschaften, alleine eine Verdopplung des E-Moduls der Binderschicht zu einer Verringerung der vertikalen Normalspannungen in der Mitte der Binder- und der Tragschicht um 4.9\% und 14.3\% führt, eine Vervierfachung des zu einer Verringerung von $11.1 \%$ und $27.6 \%$. Allerdings gibt es in beiden Fällen eine geringfügige Zunahme der Spannung in der Deckschicht. Entsprechend führt eine Erhöhung des E-Moduls der Tragschicht zu einer Erhöhung der vertikalen Normalspannungen in den Deck- und Binderschichten. Die FE Analysen zeigen außerdem, dass die kritischen Schubspannung innerhalb der Binderschicht zu liegen kommen. Außerdem konnte festgestellt werden, dass die Normalspannung in der Deckschicht unabhängig von der Radkonfiguration ist. Diese wirkt sich vor allem in der Binderschicht aus. Die Analysen zeigen, dass die ungebundene Tragschicht und Bodenschichten durch die überlappende Einwirkung von Doppel- / 
Mehrfachachsen sehr stark beansprucht werden. Hauptgrund für die Verformungen der oberen Asphaltschichten sind die vertikalen Spannungen. Die Geschwindigkeit der Verformung ist eine Funktion der Belastungshäufigkeit, der Materialeigenschaften und anderer externer Faktoren wie der Temperatur.

Im Rahmen der Arbeit wurde ein Verformungs-Modell entwickelt, das auf der Theorie von Burger beruht und die Entwicklung der Verformung während der Nutzungsdauer einer Straße für unterschiedliche Verkehrslastspektrum voraussagen kann. Das Modell berücksichtigt die stündlichen, täglichen und jährlichen Veränderungen des Verkehrs, die erwarteten Verkehrslasten und Geschwindigkeiten. Außerdem werden die über die Zeit unterschiedlichen Temperaturen in den verschiedenen Straßentiefe mit ihren Auswirkungen auf die Materialeigenschaften der Oberbauschichten berücksichtigt. Dabei zeigte sich, dass die Straße um so größeren Belastungen standhält, je ausgeprägter die viskosen Eigenschaften des Materials sind. Bestätigt wird, dass höhere Zunahmeraten der Belastung zu größeren Verformungen führen. Zusätzlich ergibt sich, dass die Geometrie des Oberbaus, insbesondere die Dicken der Schichten einen wichtigen Beitrag für die Beherrschung der gesamten dauerhaften Deformation einer Straße leistet. Es wird empfohlen, auch weiterhin an umfassenden Vorhersagemodellen für die Verformung und für andere Formen der Ermüdung / Schadensentwicklung von Asphaltoberbauten $\mathrm{zu}$ arbeiten, die die verschiedenen Lastspektren und Temperatureinwirkungen berücksichtigen können. 


\section{Dedication}

This work is dedicated to my late father Abesha Feyissa who had inspired and geared up my career. Your fatherhood, kindness, unfolded sacrifices . . . and memories of yours always remain deep in my heart!

God bless you! 


\section{Acknowledgement}

It is my pleasure to extend my sincere thanks and appreciations to those individuals who have spent their own contribution for successful completion of this research. Specially, I would like to extend sincere gratitude to my supervisors Prof. Dr. Ing. J. Stefan Bald and Prof. Dr. Ing. Michael Vormwald for their valuable guidance, encouragements and excellent comments as well as providing me all necessary facilities. I have no enough words to say except without your support this work would not have been possible.

I am indebted to thank DAAD (Deutscher Akademischer Austausch Dienst) for the financial support throughout the research period.

My thanks go to my friend Mr. Belete Kifle and his families for those unforgettable memories we have had together. Thanks to Dr. Ing. Girma Boled, our friendship has become warm and stronger than ever since then. I am indebted to acknowledge Mr. Henok Fikre for sharing valuable ideas and discussions in various issues. I never forget countrymen and women here and there for having fun around, thank you all!

I would like to thank my "Afro-Asian" office-colleagues Mr. Farag Hamed (Egypt) and Mrs. Le Thu Huyen (Vietnam) for sharing experiences, understanding and memorable friendship throughout the research period. Keep it up and remain in touch wherever you are!

I am also indebted to thank all academic and administrative staffs for Road and Pavement Engineering as well as for Material Mechanics for their efforts while making my stay memorable. Their unlimited cooperation in all matters as well as readiness and open mind for any enquiries have made me to extend my appreciations from the bottom of my heart. My sincere thanks go to Mrs. Svenja Glemser and Mrs. Theresia Glock for their valuable information and assistances which have been counted since the first day of my application.

My special gratefulness goes to my families for their support, encouragement, patience and understanding. I would like to express my appreciation to my mother Michuye Meseret for her limitless thoughts, care and love. I am also thankful to my sisters and brothers for their moral support and encouragement.

Last but not least, I would like to give my deep thanks to my wife, Tirusew Jerjebo, for her encouragement and understanding. 


\section{Table of Content}

Abstract

Zusammenfassung

Acknowledgement

xiii

Table of Content

1. Introduction

1.1. Background

1.2. Problem Statement

1.3. Research Objectives

1.4. Methodology

1.5. Organization of the Thesis

2. Review of Literatures

2.1. Overview

2.2. Traffic Characteristics 13

2.2.1. Traffic Volume and Forecasting 13

2.2.2. Traffic Classification and Distribution 15

$\begin{array}{ll}\text { 2.2.3. Vehicle Speed } & 16\end{array}$

2.2.4. Traffic Load 17

2.2.5. Contact Pressure 19

2.2.6. Effect of Heavy Vehicles and Trend of Growth Rate 20

2.2.7. Traffic Wander 23

2.3. Materials and Structural Properties $\quad 25$

2.3.1. Materials Characteristics 25

2.3.2. Structural Characteristics 28

2.3.3. Innovative Materials 28

3. Modes of Failure $\quad 31$

3.1. Rutting Failure $\quad 32$

3.2. Fatigue Failure 36

3.3. Low Temperature Cracking $\quad 37$

3.4. Asphalt Aging Failure $\quad 38$

3.5. Miscellaneous Failures 38

4. Modeling Approach $\quad 41$

4.1. The Phenomenological (S-N) Approach 41

4.2. Analytical Modeling Approach $\quad 42$

4.2.1. Continuum Mechanics 42

4.2.2. Continuum Damage Mechanics Approach 54

4.2.3. Fracture Mechanics and Cohesive-Zone Approach 57

4.2.4. Micromechanics-Based Approach $\quad 59$

4.2.5. Dissipated Energy Approach 59

4.2.6. Fatigue Endurance Limit Approach 62

4.3. Long Life Asphalt Design Concept $\quad 62$

4.4. Probabilistic Approach $\quad 69$ 
5. Thermal Behavior of Asphalt Concrete Pavement 71

$\begin{array}{ll}\text { 5.1. Thermal Response of Pavement Structure } & 71\end{array}$

$\begin{array}{ll}\text { 5.2. Thermal Properties of AC Materials } & 78\end{array}$

6. Asphalt Concrete Modeling $\quad 81$

6.1. Rut Modeling $\quad 81$

6.2. Fatigue Modeling 86

6.3. AC Finite Element Modeling 87

6.3.1. Modeling Pavement Structure with ABAQUS 91

7. FE Result Analysis and Rut Prediction Model 97

7.1. Finite Element Analysis 97

7.2. Rut Prediction Model 106

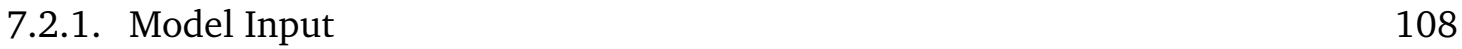

$\begin{array}{ll}\text { 7.2.2. } & \text { Program Structure } \\ & 116\end{array}$

$\begin{array}{ll}\text { 7.2.3. } \text { Model Output } & 118\end{array}$

$\begin{array}{ll}\text { 7.2.4. Verifications and Uncertainties } & 128\end{array}$

8. Conclusions and Recommendations 130

8.1. Conclusions 130

8.2. Recommendations 132

REFERENCES 134

Lists of Tables $\quad 142$

Lists of Figures $\quad 143$

$\begin{array}{ll}\text { Notations } & 146\end{array}$

Lists of Appendices $\quad 8-1$

Appendix A: Traffic and Vehicular Characteristics $\quad$ A - 1

$\begin{array}{ll}\text { Appendix B: Material Characteristics } & \text { B - } 6\end{array}$

$\begin{array}{ll}\text { Appendix C: Finite Element Analysis Results } & \text { C - } 13\end{array}$

Appendix D: Temperature Variables and Rutting Model Output $\quad$ D - 23

Appendix E: Existing Rut Prediction Models $\quad$ E - 26

Appendix F: Rut Modelling Program Flow Chart $\quad$ F - 28 


\section{Introduction}

\subsection{Background}

A road pavement is a structure of superimposed layers of selected and processed materials that are placed on the basement soil or subgrade. Flexible pavements constructed for heavy duty vehicles are composed of asphaltic layers (wearing, binder and base courses) and sound subbase layer which laid over a well compacted and strong subgrade foundation. The main structural functions of a pavement are to support the wheel loads applied to the carriageway and ultimately distribute them to the underlying subgrade layer. The pavement is designed and constructed based on the most economical combination of layers that guarantees adequate dispersion of the incident wheel stresses so that each layer in the pavement does not become overstressed during the design life of the highway.

Asphalt concrete (AC) pavement is one of the largest infrastructure components in civil construction works and complex system that involves multiple layers of different materials subjected to various combinations of irregular traffic loadings and varying environmental conditions. It provides the road-user a smooth, quiet and skid-resistant riding surface, maximizes tire contact by providing more traction, and saves wear and tear on vehicles. AC pavements are safe, economical and most long-lasting roads that can be built very quickly thereby reducing costs due to traffic delays and save the traveling time. In addition, asphalt pavements are purely recyclable that old AC pavements can be reprocessed, remixed with a portion of fresh materials and used again and again.

It has been universally witnessed that both traffic volume and loads on roads are going on increasing from year to year with alarming rate all over the world. Such heavy traffic growth demands better performance roads for efficient transport of commercial and industrial products without delay. The repetitive traffic loading that the road experiences during its service life combined with environmental factors causes deformation, fatigue cracking, instability and other forms of deterioration which ultimately degrade the serviceability and durability of pavement structures.

Estimation and prediction of the maximum distresses which are likely to happen within the pavement layer during the pavement service life is most vital and a significant factor for proper design of the pavement. Analytical models play here an important role to foresee the distressdeformation behavior of the pavement. Constitutive modeling of the deformation behavior of asphalt concrete enables better understanding of the deterioration mechanism in the pavement structures and aid to design most economical and sustainable roads. Many models have been developed so far for asphalt concrete to estimate the number of repetitions to failure in the rut/fatigue mode. A well constructed flexible pavement that is built above well defined threshold strength has a sustainable structural life provided that the distresses, in the form of fatigue, ruts and other modes of failures are kept at minimum value in such away that they do not affect structural integrity of the road.

On the other hand, better understanding of materials behaviors and structural responses of asphalt concrete in combination with load-stress-strain computation tools, allows for optimization of asphalt pavement thickness and material choice. The structural design of a road pavement is a balance of a number of requirements based on predictions of future traffic 
volumes (including axle loads, tire pressures), properties of constituent layers, quality of construction materials, climatic as well as environmental conditions and construction techniques.

For the past many years, many pavement structural design procedures use an empirical approach where the relationship between design inputs and pavement failures were determined using past experiences, experimentations or combination of both. The structural strength, which usually is expressed in terms of structural number, along with the predicted loading, reliability and serviceability of the pavement are the necessary inputs. This method is an iterative process which typically gives the output either in terms of equivalent single axle load or the structural number which is associated with pavement layer thickness. In this method of pavement design, the asphalt concrete mixtures, subgrade and other structural materials behaviors have been characterized using conventional laboratory test methods in order to determine their properties and study the effect of rate of loading, temperature and materials properties on mix behavior. This trend of material characterization and design procedure has been replaced by advanced mechanistic-empirical technique which describes and models the relationship between the pavement responses of stresses, strains and deflections with that of the physical causes of loads, material properties and the environmental conditions. The empirical part of the design uses the pavement response to predict the life of the pavement on the bases of the actual field performance.

Extensive research has been in progress worldwide including costly accelerated dynamic fieldtesting to replace and modernize the existing conventional design methods. Different analysis and design techniques are under progress based on some basic mathematical and material models. The primary methods of modeling and analysis reported in the literatures include elastic, viscoelasticity, elasto-plastic, visco-plasticity and finite element. Many researchers employed linear and nonlinear elastic and viscoelastic mechanical models to determine experimentally the constitutive parameters for asphalt concrete. Several factors, such as rate of loading, temperature and moisture are considered in many modeling techniques and applied to asphalt paving mixtures and flexible pavements when investigating permanent deformation, fatigue cracking and other forms of damages.

In reality, flexible pavements are subjected to dynamic loading caused by truck traffic. The mechanical damage to the pavements due to such loading cannot be estimated without the laboratory characterization of AC for a range of loading of various durations that pavements experience in service. In general, laboratory test results in conjunction with the theoretical predictions will permit comparison with actual pavement performance available from field measurements. The findings from such studies will ultimately lead to adoption and use of new or improved materials based on satisfactory performance.

Most economical and best performing pavement can be achieved when the supportive capacity of layers exceeds the distress transmitted from the carriageway. Major variables affecting the design of a pavement are traffic characteristics, the surrounding environmental conditions, material and structural behaviors of the pavement. Hence different forms of damages caused by various factors are required to be taken in to account in pavement design, so that associated damages are kept to the minimum possible to provide the pavement a sustainable and long service life.

Durable road pavement structure can be built by proper choice of construction materials, appropriate blending of each constituents and selection of mix properties with respect to stiffness 
modulus/strength, durability, flexibility as well as thickness of pavement layers which has the capability to perform well under adverse conditions without significant loss in stability during the intended service period. Longer design life for flexible pavements, which carry the anticipated heaviest volumes of traffic, will yield a lower whole life cost, less traffic delay as well as better economic growth for one nation. This requires strategies for design that decrease the need for regular maintenance and rehabilitation work thereby cause less disruption to the road user. It is beneficial for road sector to design and construct a reliable and durable road in order to save overall construction and operational costs. On this research, it is intended to identify critical parameters contributing to AC damage and develop a model for asphalt concrete road that predicts the damage accumulation and serves the highway community to design a pavement for extended period of time.

\subsection{Problem Statement}

Asphalt roads are designed and constructed to serve the upcoming traffic that reveal during the service life of the road. Different factors taken in to account in the design and construction of AC pavements include the characteristics of the traffic, climatic conditions, material as well as structural properties and other elements which have significant impact on the overall performance of the road.

Different design and construction techniques are developed and standardized by many agencies and nations. These standards present policies, detailed procedures and analysis parameters and criteria which enable designers to design the pavement structural layers, present findings and recommendations in a systematic manner based on sound engineering principles consistent with the expectations and practices of the country. According to the AASHTO Road Design Manual [38], for example, a pavement is designed based on the resilient modulus of the road bed soil and the structural number (SN) of the super structure which is considered as the strength measure of pavement that would carry the mixed vehicle loads over the roadbed soil, while providing satisfactory serviceability during the design period. The German design standard specification [RSTO 1] recommends design methods according to the road category, traffic intensity, characteristics of the asphalt layers, resilient modulus of the subgrade soil, the properties of the materials to be used for construction and other environmental condition such as the possible formation of ice within the soil layer and the temperature of the region where construction is being done. The Ethiopian Road Authority (ERA) manual adopted [36] a California Bearing ratio (CBR) method for the design of a flexible pavement. Likewise the Indian Road Congress (IRC) manual follows the same trend.

The pavement can no longer absorb and transmit the wheel loading through the road structure when the layers have failed for various reasons, as through aging, inadequate design, poor construction and maintenance practices, low bearing capacity of the materials or the gradual degradation of the strength of the road due to increased traffic flow and load and/or due severe climatic conditions, decreasing subgrade strength in conjunction with inadequate surface/subsurface drainage facilities and so on.

As well known, asphalt mixtures are multi-phased composites consisting of irregularly shaped and randomly oriented aggregate particles embedded in an inelastic matrix that is known to 
degrade with time and under mechanical, thermal, and environmental loads. The asphalt mixtures generally exhibit complicated mechanical behavior with numerous damage modes.

When the load is removed the pavement layers rebound, however, with very small amount of deformation which could stay permanently and accumulate over many load repetitions causing rutting in the wheel path. Permanent deformation (rutting) of asphalt pavements is one of the well-recognized road surface distresses in asphalt concrete pavements that reduces the useful service life the pavement, affects vehicle handling characteristics and creates serious hazards for highway users. In many parts of the world, the major concern is the excessive deformation in heavy duty asphalt-concrete pavements resulting from frequent repetitions of heavy axle loads, many of which are operating with high tires pressures. Roads that develop longitudinal ruts can lead to accumulation of water along the wheel track which accelerates the deterioration further as well as causes loss of control of vehicle maneuvering during lane changes and threaten the safety of road users.

Pavement distress resulting from fatigue of asphalt concrete pavements has been acknowledged as the most costly form of distress to correct through rehabilitation. Studies indicated that environmental conditions, tire-pavement interactions, mixture characteristics, aging of binder and construction practices are among the factors for the occurrence of the damage. The cumulative fatigue damage due to loading causes the elastic stiffness modulus to decrease with the number of load repetitions.

Various tests conducted revealed that asphalt concrete materials display different behaviors at different testing conditions. In addition, experimental observations indicated the existence of a time lag between the response of the applied load and the resulting deflection implying that the material has time-dependant behavior. It has been clearly stated that asphaltic materials are dependent on rate of loading and temperature. AC behavior varies from elastic and linear viscoelastic at low temperatures and/or fast loading rates to nonlinear visco-elastic, viscoplastic, and plastic at high temperatures and/or slow loading rate. Many roads experience extreme daily low temperatures during the winter and high temperatures and solar radiation during the summer which causes thermal cracking and permanent deformation. At high temperature the viscous and the plastic characteristics of the AC material control the deformation behavior of the asphalt layer which leads accelerated accumulation of rutting along the wheel path.

A reliable mechanistic pavement design procedure has to predict pavement response and the development of pavement distress (such as rutting and fatigue cracking) under various traffic and environmental conditions. Over the course of the past several years, various methods have been developed to determine the necessary thickness of an asphalt pavement, each having its advantages and disadvantages, with each design method being correct when applied within its select variables or criteria. It is hard to say that specific design technique is absolutely addressing and solving the real problem of the road that designers, constructors and road users are currently facing. Handful problems have been mentioned in various workshops, seminars, literatures and other media and at the same time possible remedies have been suggested to alleviate the existing problems. So far many rut, fatigue and thermal cracking models development are under progress. But the current trend developing a model by singling out specific parameter by itself is not a solution for the real challenge the design and construction industries are facing as it 
requires to implement and simulate it for the actual pavement structure that experience not only traffic load but also thermal and other environmental load combinations at different time frame.

Pavement design is continually evolving from remarkable empirical processes developed 40 to 60 years ago. These involved observations of how pavements interacted with soils, climates and levels of traffic and then determining the pavement thickness on bases of these observations and experiences. While empirical methods are still widely used, the design and construction industry have further moved towards better scientific approach of mechanics-empirical based methods to overcome the challenges and the short comings of the previous method. Although such advancement is appreciable and welcomed in many standards, at the same time it is underlined that a lot of work remains undone to resolve the difficulties and challenges that the design industries are still facing to design and suitably enhance the serviceability of a road for an extended period of time under multitude loading and environmental conditions.

Many mathematical models have been developed to assess and simulate the reactions of the asphalt concrete to different loads under specific conditions. However the reality shows that a pavement is subjected to a wide range of load intensity and characteristics as well as temperature and other environmental fluctuations. On the other hand past experience indicated that some pavements constructed for a design period of 20 to 25 years have been observed serving for more than the predicted service life. It is such discrepancies that motivate further investigation and evaluation of the behavior of a pavement under different situations. Therefore finding a solution to these issues is a major step towards improving the quality of pavement analysis and design both for the researcher and the practitioner.

In this information age as problems get easily manipulated and solved, it is required to work out possible mode of failures and corresponding remedies in one umbrella with the actual loading and environmental conditions. Therefore, a realistic prediction of the performance of asphalt pavements does not remain as one of the most challenging tasks for pavement engineers. Rather the service life of asphalt concrete pavements can be predicted and further enhanced in such a way that the pavement will remain for extended period of time and last longer than the traditional design period. This is possible when the response of the pavement can be properly evaluated under the prevailing circumstances of loading and environmental conditions.

\subsection{Research Objectives}

The main objectives of this research work are:

- Evaluate the performance of the AC pavement when subjected to different expected axle load magnitudes and estimate various distress forms associated with them. Assess the repetitive action of load and its rate of application on the performance as well as the durability of the pavement.

- Review theoretical models as well as existing models for permanent deformation and fatigue damage of asphalt concrete mixtures.

- Analyze and formulate the climatic (daily, monthly and annual change) and environmental impact on the deterioration of AC pavement performance, elaborate the 
degree of deterioration with respect to temperature and evaluate the cyclic effect of climate during the service period of the AC pavement.

- Estimate the combined effect of the above conditions, evaluate pavement performance under such combinations and identify dominant parameters greatly influencing such performances.

- Develop a promising model that analyzes and predicts long lasting pavement performance under the given and expected traffic and environmental conditions.

- Propose methodologies and design approaches for design of sustainable and long lasting asphalt concrete road.

\subsection{Methodology}

Accomplishment of the research goal required the review of applicable practices, research findings, information on hot mix asphalt (HMA) mixtures and pavement performance models. Relevant literatures on current design approaches, various modes of failure and damage propagations have been reviewed. The methodologies adopted to achieve the objectives are outlined as follows.

1. Theoretical material modeling approaches and several load-damage models developed so far based on continuum damage mechanics, cohesive-zone, Micromechanics-based approaches and other possible methods to simulate failure behavior of asphalt concrete mixes are studied. In this research, currently available models, developed based on the basic modeling approach to describe the performance and distress characteristics asphalt concrete, are studied. The dominant factors (asphalt concrete properties, climate, traffic loading) that are likely to influence the structural deformation of asphalt concrete are also reviewed. Degrees of influence of several variables, which have been taken into account in various models are evaluated.

2. The traffic characteristics, distribution and density of vehicles across lane/road, speed and time space behaviors are studied and analyzed, their influence on pavement response is evaluated and critical distress forms and corresponding locations are identified.

3. Current modeling approaches and shortcomings to simulate the response of asphalt concrete pavement when subjected to traffic, thermal loading and other environmental effects are evaluated.

4. The time, load and strength dependent response of the stress-strain behavior of the different structural layers are analyzed using ABAQUS finite element software and the stress pavement responses are used as an input for a newly developed prediction model.

5. The impact of temperature on the material properties as well as on pavement performance is assessed, and the response of the pavement and the modes of failure with change in structural characteristics and alteration of materials' behaviors under different traffic loading and/or thermal conditions are studied.

6. A rut prediction model is developed and programmed while taking into account the combination of loading and thermal fluctuation. Critical parameters dominantly 
accelerating and contributing to rutting damage are identified and remedial solutions are forwarded.

\subsection{Organization of the Thesis}

The thesis report is composed of eight chapters. In the introductory chapter, the background of the thesis and the objectives of the research are explained. The methodology followed to accomplish the aim of the research is also explained in this chapter.

The second chapter focuses on the literature review and the possible modes of damage in flexible pavements with highlights on the rutting and fatigue caused by traffic and thermal loading in asphalt layers. Various vehicle types, characteristics and corresponding contribution for the deterioration of flexible pavement are also explained. In this chapter, a due concern has been given to the traffic composition of various categories of vehicular traffic with different performance and operational characteristics. This chapter also elaborates the roles of the materials and structural properties in pavement performance.

The third chapter deals with the different modes of failure in asphalt concrete pavement. Rutting and fatigue failure and associated causes are explained. In addition, other modes of failures including low temperature cracking, aging and other miscellaneous failures are discussed.

The forth chapter explains the theoretical modeling approaches commonly adopted for many engineering applications. The phenomenological tools as well as analytical ones such as elastic, viscoelastic, viscoplastic, continuum damage mechanics and other applicable asphalt concrete models are discussed in this chapter. The concept of long life asphalt concrete pavement is also introduced in this chapter.

The fifth chapter has given a due concern on the thermal behavior of asphalt concrete pavement and its contribution to damage and associated modes of failures. The daily and annual temperature variations, temperature gradient across the depth and applicable models to predict the thermal response of the road structure have been assessed.

The sixth chapter particularly focuses on the rutting and finite element (FE) modeling of asphalt concrete pavement. Mechanistic-empirical rutting model and other rutting models adopted for evaluating permanent deformation in flexible pavements are explained. The progress of development and Finite Element implementation of a constitutive model with damage in AC pavements are also elaborated in this chapter.

The seventh chapter explains the finite element analysis of asphalt concrete model and the program developed to predict the performance of asphalt concrete pavement. FE stress-strain outputs with varying loading conditions, structural geometry and material properties are discussed in this chapter. In addition, the model developed to predict the rutting progress for the prevailing traffic loading, temperature changes of the surrounding and results of the model output are discussed.

The last chapter outlines the conclusion and the recommendation for further works. 


\section{Review of Literatures}

\subsection{Overview}

A road pavement is a structure of superimposed layers of selected and processed materials that are placed over the basement soil. The main structural function of a pavement is to support the wheel loads applied to the carriageway, distribute them to the underlying subgrade and to provide a uniform skid-resistant running surface with adequate service life. Pavement design is concerned with developing the most economical combination of pavement layers that ensure the stresses and strains transmitted from the carriageway do not exceed the supportive capacity of each layer during the design life of the road. Selection of layer materials and thicknesses requires evaluation of various design strategies developed from consideration of a wide range of factors. Major variables affecting the design of a pavement are the volume and composition of traffic, the environment, the materials strength characteristics within the pavement layers and the thickness of each layer. These variables are the inputs used to determine the thickness and characteristics of the structural layers of a pavement either empirically or analytically.

The estimation of traffic levels is the most important parameter for the structural design of any road pavement. A particular importance for asphalt concrete pavement is the estimation of commercial vehicle volumes. The dynamic loads generated by heavy vehicles are considered as the primary cause for road structural damages. When such loadings are excited by roughness of the road surface they result in additional dynamic stresses and strains in pavements [Cebon 1993] which accelerate road surface deteriorations. Uneven axle load sharing can also result in unnecessarily high average tire forces with consequently high stresses-strains in the road surface and additional road damage which increases fatigue damage since the power-law damage relationships accentuate the effects of more heavily loaded axles. Each traffic load application contributes to some extent to pavement distresses. Heavy vehicles particularly contribute the greater portion of the deterioration of flexible pavements. The vertical and horizontal forces that the truck imposes on the pavement surface play a role in many different deterioration modes such as:

- Structural, surface and reflexive cracking

- Rutting in bituminous layers and the subgrade

- Longitudinal unevenness

- Raveling and loss of skid resistance etc.

When one or more of these distresses reach a certain unacceptable level, the pavement is considered as failed. Better understanding these problems would possibly reduce the road damage caused by various factors and associated costs. This may be achieved by highway authorities, through improved design and construction of roads; or by government agencies, through regulations intended to encourage the use of more 'road-friendly' vehicles, better performing road materials and construction techniques.

In addition to loading characteristics, operational behaviors of vehicles have great impact on the performance of a pavement. Road materials and structural responses are sensitive to vehicle speed [Cebon 1993] and to the frequency content of the applied loads. As the speed increases, the peak strain under a constant moving load diminishes in amplitude and occurs behind the point of application of the load. This 'speed effect' is important when considering the road damage caused by dynamic tire forces, as rutting and fatigue damages incurred by road materials are very sensitive to strain amplitude.

In addition, road construction materials and road surface responses are extremely sensitive to environmental [Cebon 1993] factors particularly moisture and temperature. Environmental variations can have a significant impact on pavement materials and the underlying subgrade, which in turn can drastically affect pavement performance. Frost heaving of soil caused by crystallization of ice within the soil voids usually in the form of continuous ice lenses, layers or 
other ice masses changes the physical properties of the subgrade soil and brings about heaving on the overlying soil and pavement layer which ultimately result in crack, roughness on the pavement. On the other hand as the ice melts and turns to liquid it cannot drain out of the soil fast enough and thus the subgrade becomes substantially weaker (less stiff) and tends to lose bearing capacity.

Further, extreme temperature variations can cause severe pavement damage due to expansion and contraction of the pavement. It is one of the most dominant factors that impact the variation in modulus of asphalt concrete layers [Shen and Kirkner 2001]. It also affects the rheology of asphalt binder, mixture characteristics and the performance of asphalt concrete pavement throughout the design life.

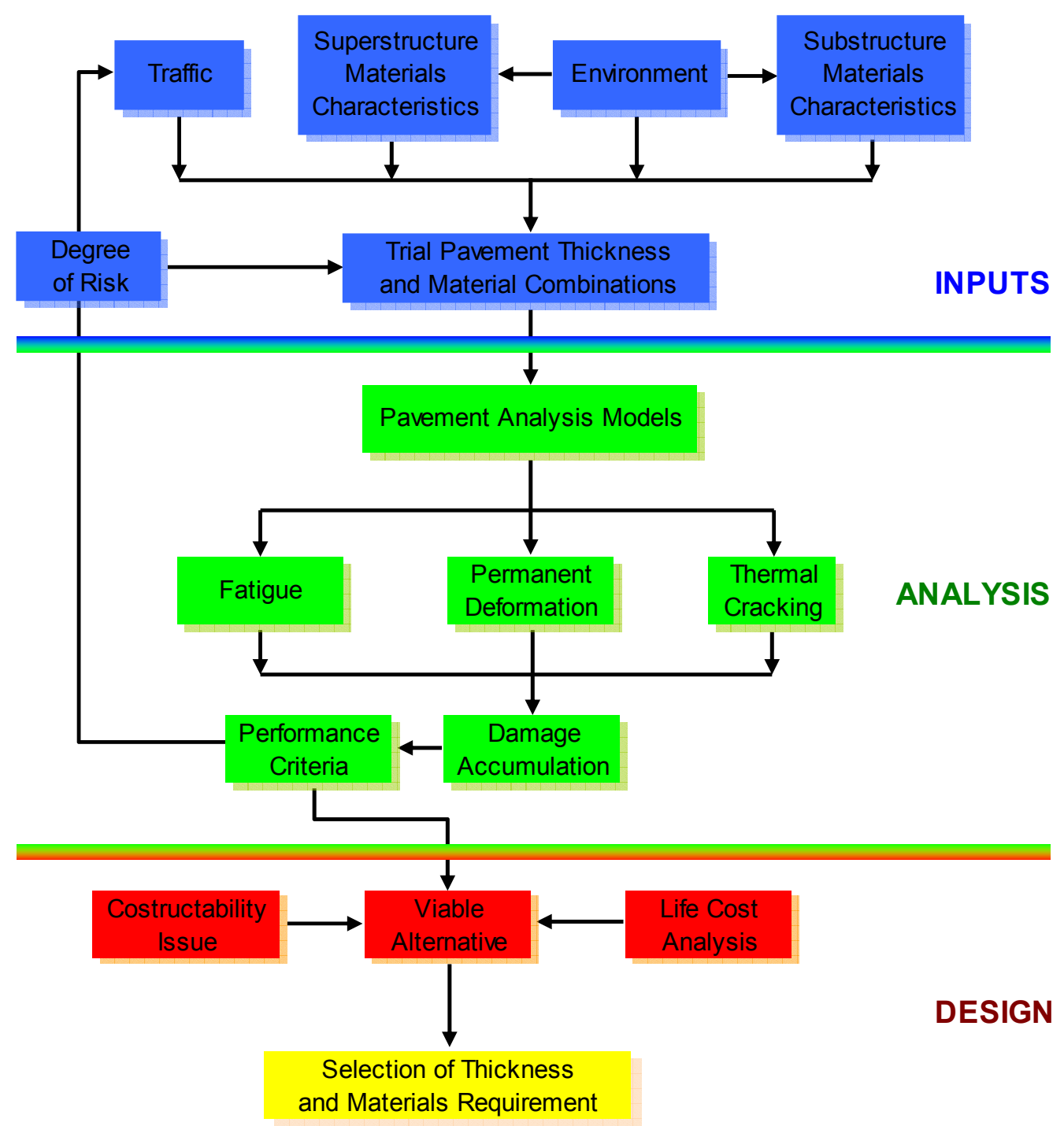

Figure 2.1 Simplified AC pavement analysis and design strategy [modified, Ref. 38]

Pavement material properties are constantly changing over time due to load, influence of climate, as well as the onset of fracture or deformation. It is quite important to consider these changes in any pavement analysis and design. Materials that are subjected to load-related fatigue distress may experience a severe degradation of properties with time and load repetitions which gradually develop micro-cracks that lead to a reduction of elastic modulus. This reduction in turn, leads to an increase in stress states within the pavement and a greater possibility of permanent deformation. 
An asphalt pavement, when designed and constructed properly, can provide years of service but at the same time continually undergo various types of distresses that induce minor defects into the pavement due to loading, temperature, moisture and other factors which eventually lead to the pavement's failure. The basic requirement for asphalt concrete pavement design is that it should be of sufficient thickness to spread the surface loading to stress-strain intensity that the underlying layer is able to withstand and prevent damage due to various reasons.

Road design methods have been empirical based, with the performance of pavements being analyzed and design charts being compiled based on the information obtained from the on-site observations and specially constructed test sections. Such structural design approach is being somehow outdated from engineering principles and hardly copes with variables or factors beyond those included in the trial section on which the design method is based. These methods are being replaced by analytical techniques that involve

- Characterization of the pavement layers and subgrade to meet the requirements of a theoretical model

- Computation of parameters considered to have a primary influence on selected aspects of pavement performance and

- Utilization of these parameters in performance models to evaluate the structural adequacy of the pavement under consideration.

In more recent years analytical or 'mechanistic' road design procedures have been advocated and used in many countries. These methods are based on determining the actual loading stress and strain relationships, the engineering properties of the asphalt material and some specific climatic conditions. A mechanistic design method computes the stresses and strains in pavement in response to vehicle loads. The stresses and strains are used to estimate the amount of damage done to the pavement by vehicle loads which eventually determines how long a pavement can last. These procedures provide the background needed to analyze the road damaging potential of heavy vehicles.

Ultimate pavement performance is related to durability, strength, stability, stiffness, flexibility and resistance to fatigue and rutting. This requires a thorough understanding of materials and structural properties that affect pavement stability and durability which needs the use of mechanical test methods to measure particular properties in order to meet desired performancerelated specifications. Laboratory test results in conjunction with the theoretical predictions will permit comparison with actual pavement performance available from field measurements. The findings from such studies can ultimately lead to adoption and use of new or improved materials based on satisfactory performance.

Performance models attempt to relate material parameters associated with a particular mode of deterioration to the number of repetitions that can be sustained before a terminal condition is reached. Several factors, such as rate of loading, temperature and moisture are considered in modeling techniques applied to asphalt paving mixtures and flexible pavements when investigating pavement distresses. The two most important distress forms governing the asphalt design process are permanent deformation and fatigue cracking. Current mechanistic pavement design practices are attempting to optimize resistance to fatigue and rutting. Designers often attempt to minimize deformation of the granular layers by limiting the stresses or strains at critical pavement sections. In some design procedures, the elastic deflections of the surface or the sum of the permanent deformations of each layer are used as design criteria. Experimental fatigue and permanent deformation characteristics of the road materials are combined with the calculated primary responses to estimate the service life. Suitable pavement layer thicknesses and material property specifications are then determined by iteration.

The subgrade and asphalt concrete layers rutting are two basic types of rutting. The permanent strains of each layer are accumulated over a period of time leading consequently the pavement to structurally and functionally unsuitable state. Most failure criteria have been attributed mostly to 
the weak pavement structure (subgrade) in terms of the vertical compressive strain to the number of cycles to failure. At elevated temperatures, AC mixes exhibit remarkably different volume-change and shape-distortion deformation modes in terms of their sensitivity to temperature, rate of loading, and residual permanent deformation. Mechanistic methodologies have recently been developed for permanent deformation characterization of asphalt paving materials on the bases of elastic or rheological properties of hot mix asphalt and included in the design techniques.

Fatigue in AC layer is one of the criteria for flexible pavement design. Fatigue failure of the AC layer is related to the magnitude of tensile strains at the bottom of the layer. The magnitude of the flexural strains in the $\mathrm{AC}$ is dependent on the wheel loading conditions, thickness of the paving material layers, the properties of the various paving layers and the subgrade soil. Fatigue transfer functions relate the number of load repetitions to reach certain pavement cracking failure conditions (i.e., crack initiation, 10 percent cracking area, etc.) to the maximum tensile strain in the AC layer. It is therefore important to measure AC strains so that pavement response and cracking performance can be properly evaluated.

Most of the design procedures that are used in road engineering are based on linear elastic multilayer analysis and laboratory tests. Relatively few of the primary response models have been validated by comparison with field measurements. It is necessary to have qualitative agreement between measured and computed strains in a layered elastic system subjected to vertical and longitudinal static surface loads. Unfortunately, design life and material degradation, which are essential issues in estimating the life time of a pavement, can not be addressed only by linear elasticity. In the damage accumulation process the complex nature of asphalt concrete is essential. Accurate performance predictions are possible only when that nature of the material is properly modeled. The temperature and strain rate dependency as well as the state of stress dependency of the material response must also be taken into account. When it comes to failure predictions, this aspect of the material behavior can no longer be neglected.

AC can be characterized by a certain level of rigidity of an elastic solid, but at the same time, flows and dissipates energy by frictional losses as a viscous fluid. When a vehicle travels on top of an asphalt concrete pavement layer, three different types of strain develop in the material: elastic recoverable strain, viscous recoverable and irrecoverable strain. Elastic strain is associated with the ability of AC to respond to the applied load and to recover instantaneously the energy released during deformation. On the other hand, viscous strain involves the time-dependent deformation of AC. Only part of the viscous strain is recoverable and time-dependent. To avoid premature failure of pavement systems, an analysis procedure that can accurately predict pavement response to different vehicular loading and material properties is preferably be used.

In recent years there has been a proliferation of computerized pavement analysis systems [Cebon 1993] which use elastic layer theory for flexible pavement design and life prediction. Comprehensive review of computer packages for calculating static and/or dynamic asphalt concrete pavement responses and predicting distresses (damages) have been presented and under progress by many researchers [Ali et al. 2009, Blab and Harvey 2002, Park et al. 2005, Saad et al. 2006, Siddharthan et al. 2002].

The finite element method (FEM) has also been used extensively and is particularly useful for analysis of pavements which can be prone to thermal loading as well as being subject to vehicle loads. In the FEM analysis of AC pavement, the layers are meshed in such a way that the region of interest is divided into small discrete elements, material properties are assigned and the model is submitted for analysis with the application of load and imposed boundary conditions. The intensity of internally distributed stress within the pavement structure, strains and deflection at various points are the expected output. Of particular interest, the critical pavement response variables required include:

- Tensile horizontal strain at the bottom or top of the HMA layer (for HMA fatigue cracking) 
- Compressive vertical stresses and strains within the HMA layer (for HMA rutting)

- Compressive vertical stresses and strains within the base and subbase layers (for rutting of unbound base layers)

- Compressive vertical stresses and strains at the top of the subgrade (for subgrade rutting)

Estimation of the likely loading types and environmental conditions play an important role for design and construction of sustainable road. In general, well-constructed pavements built above a minimum strength are not likely to exhibit structural damage when subjected to very wide range of commercial traffic for a long period time, provided that deterioration originating in the asphalt concrete such as either rutting or surface initiated cracking are detected and remedied before they have serious impacts on the structural integrity of the road. There are a number of case studies which support such conclusion indicating the presence of extended life a pavement structure [Soltani et al. 2006]. It is highly regarded very useful and economical to consider the design of pavements on the bases of long service life which can be achieved by thoroughly identifying the complex response of a pavement developed due to a combination of different load and environmental actors.

\subsection{Traffic Characteristics}

The design of a highway and its features are based upon explicit consideration of the traffic volumes and their characteristics. Traffic data for a road or section of road are generally available or can be obtained from field studies which include traffic volumes for days of the year and time of the day and the distribution of vehicles by type and weight. The data also include information on trends from which the designer may estimate the traffic to be expected in the future.

The road traffic is composed of various categories of vehicles with different performance and operational characteristics. A given flow of traffic through streets and highways of defined characteristics varies by location and time. Study of traffic characteristics is the most essential prerequisite for any improvement of traffic facilities. It is required by the pavement designer to associate the damaging effects of the applications of an axle of any load applied to the pavement. The volume and distribution of vehicles types are also important to estimate the pavements anticipated life. A full characterization of the traffic loading on pavements would require information about parameters that include

- Composition of vehicles and proportions of heavy trucks

- Dynamic load spectrum, single, tandem and triple axle load combinations in this spectrum

- Load distribution over each axle, within tandem or tridem axle combinations

- Tire type, contact area, tire inflation and contact pressures

- Distribution of vertical, horizontal tire/pavement contact stress

- Lateral distribution of the wheel loads with in the traffic lane

Highway system accommodates a wide variety of sizes and types of vehicles, from the smallest subcompact passenger car to the largest double and triple tractor trailer combinations. The operating and performance characteristics of these vehicles vary almost as much as their size and weight. Most of these characteristics have been considered in highway design.

\subsubsection{Traffic Volume and Forecasting}

Traffic volume is the number of vehicles crossing a section of road per unit time at any selected period. It is a parameter most often used as a quantity measure of flow, commonly expressed in terms of vehicles per day and vehicles per hour. Traffic volume is generally accepted as a true measure of the relative importance of roads in deciding the priority for improvement and expansion. A complete traffic volume study may include the classified volume study by recording the volume of various types and classes of traffic, the distribution by direction and turning 
movements and the distribution on different lanes per unit time. Classified volume study is useful in structural design of pavements, in geometric design and in computing roadway capacity.

When designing a new road pavement or rehabilitating an existing one, the measurement of current traffic levels is an important step in the process followed by estimating the amount of future traffic that the pavement can carry over its design life. Traffic predictions are based on historic records of traffic volumes on roads/facilities and the percentage of the traffic. It has been universally witnessed that both traffic volume and loads on roads are going on increasing from year to year with alarming rate all over the world. In most cases, highways classified as principal arterial or interstate will have exponential growth. The first parameter needed to estimate the traffic effect is the total volume of the average daily traffic (ADT), which can be measured by using pneumatic tube over a number of days or estimated from a traffic map for a similar road. ADT is the most fundamental traffic variable used for highway planning, design and maintenance. For design purposes the measured values are converted to annual average daily traffic (AADT) using daily and seasonal factors specific to that area. Daily volumes generally are not differentiated by direction or lane but are totals for the entire facility at the specified location.

Likely traffic growth over the lifetime of a road project is an important consideration in pavement design. The estimation of traffic volume throughout design period is based on the growth rate. It is a vital component of life cycle analysis as it has a high influence on the assessment of the damages attributable to road.

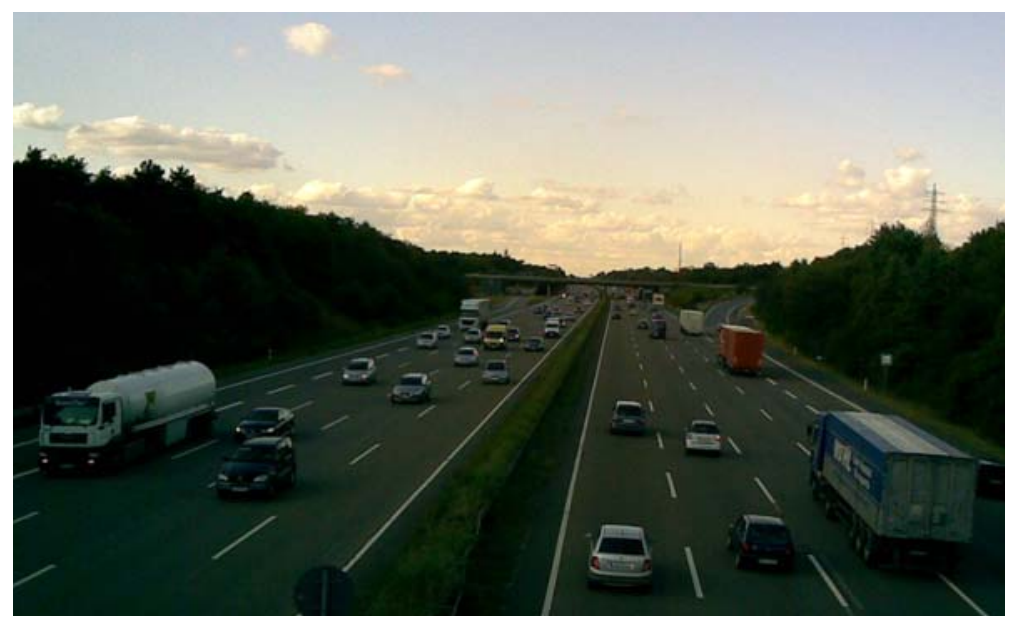

Figure 2.2 Traffic flow characteristics

The current ADT volume for a highway can be readily determined when continuous traffic counts are available. When only periodic counts are taken, the ADT volume can be estimated by adjusting the periodic counts according to such factors as the season, month, or day of week. A growth rate estimate is required to convert a single year traffic count into the total traffic which will be experienced over the pavement design life. If an AADT and growth rate is provided, then a future AADT can be calculated using the following commonly used equation.

$$
A A D T_{F}=A A D T_{C}(1+r)^{N}
$$

Where $\mathrm{AADT}_{\mathrm{F}}$ - the annual average daily traffic for the future year

$\mathrm{AADT}_{\mathrm{C}^{-}}$the annual average daily traffic for the initial year

$\mathrm{r}$ - annual traffic growth rate 


$$
\mathrm{N} \text { - design life of the pavement }
$$

The total commercial vehicles in a lane for a given time span are estimated using the following equation.

$$
T_{n}=365 F_{0}\left[\frac{\left((1+r)^{n}-1\right)}{r}\right] P
$$

Where: $\mathrm{T}_{\mathrm{n}}$ - Total number of commercial vehicles over the $n$ years design life

$\mathrm{F}_{0}$ - Initial daily flow (base year)

$\mathrm{r}$ - Commercial vehicle growth rate

$\mathrm{n}$ - Design life

$\mathrm{P}$ - Proportion of commercial vehicles in a lane

\subsubsection{Traffic Classification and Distribution}

Vehicle classification is an essential aspect of traffic volume evaluation (as well as evaluation of equivalent axle loads). Typical classification of volume by components of traffic stream include directional and lane distribution, vehicle types and axle loads, turning movements and vehicle occupancy which are important in the planning, design and operation of traffic facilities.

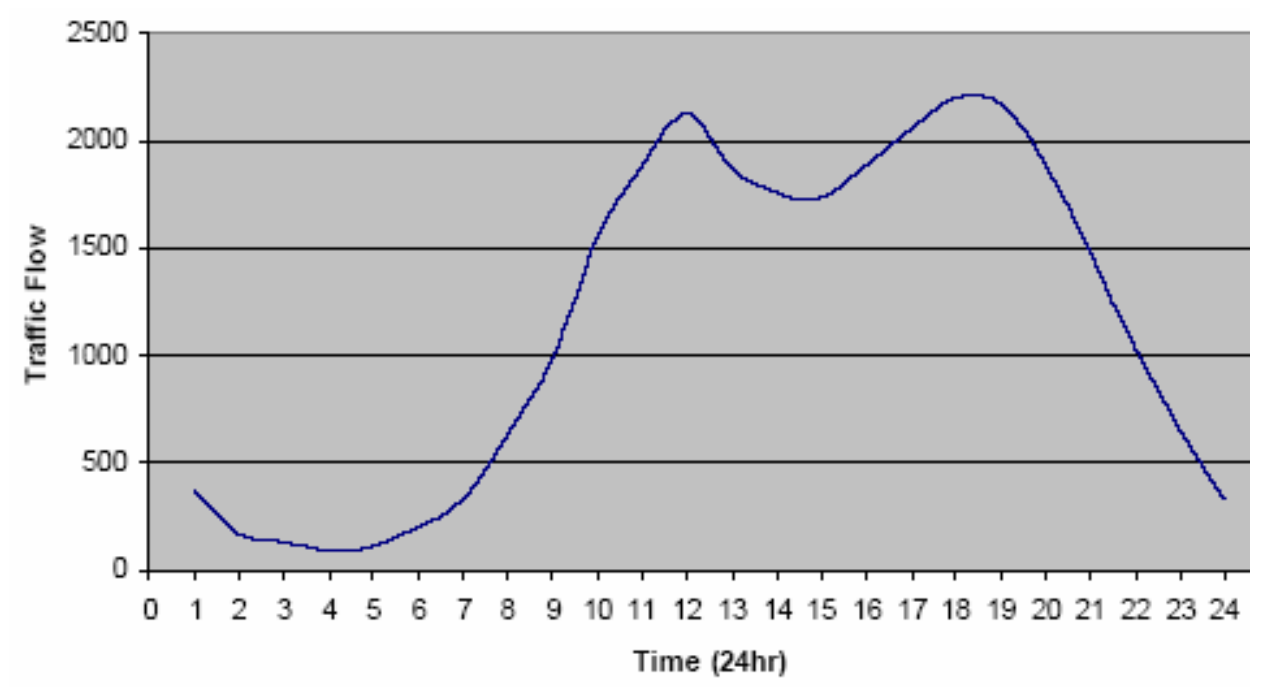

Figure 2.3 Traffic hourly variations [54]

Along with load type and repetitions, the load distributions across a particular pavement can also be estimated. The total number of loads is probably not distributed exactly equal in both directions. Often one direction carries more loads than the other. Furthermore, within a direction, the outer most lanes carry mostly the trucks as well as slow moving vehicles and therefore subjected to the heaviest loading. So, pavement structural design should account for these types of unequal load distribution. The loads expected in the design lane are either directly counted or calculated from the cumulative two-direction loads by applying factors for directional distribution and lane distribution.

Traffic flows often have large hourly, daily, weekly, monthly and seasonal variations. Daily volumes are frequently used as a basis for highway planning and for general observation trends but they haven't been used alone for design or operational analysis purposes. Volumes vary considerably during the course of a 24-hr. The hourly volume is in general utilized for many forms of traffic design and analysis purposes but it would have been very important if it could be used for a pavement structural design. The majority of the traffic travels between approximately 05:00 and 21:00 hours. 


\subsubsection{Vehicle Speed}

The actual speed of vehicles over a particular route may fluctuate widely depending on several factors such as geometric features, terrain, traffic conditions, time of travel, place, environment and driver behavior. Congestion during peak time periods may significantly reduce the overall vehicular speed of a facility. Further, truck speed and travel-time reliability are affected by infrastructure design and capacity, incidents and work zones. Heavy trucks generally require a longer time to accelerate and decelerate than do passenger vehicles.
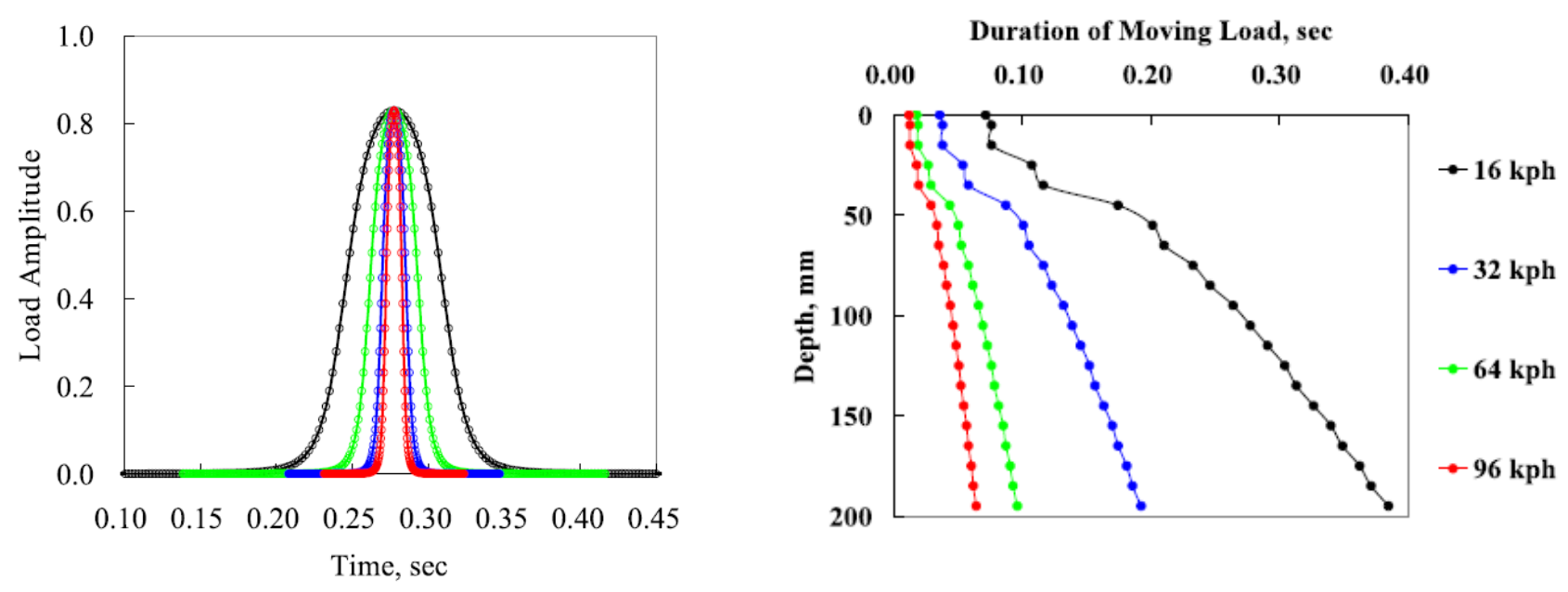

Figure 2.4 Dependency of the duration of moving load on vehicle speed and depth [Yin et al. 2008]

There is a decrease in pavement deflections with increasing vehicle speed. This is most evidenced in pavements carrying vehicles at very low speeds. High speeds result in a reduction in the time that a moving wheel load 'rests' on the road pavement, and this short-time exposure can reduce fatigue and rutting of the viscoelastic materials in a flexible pavement. The strength of pavement materials and subgrade depend upon the rate at which they are loaded, with the measured modulus increasing with increased rates of loading. In general, slower speeds and stop-and-go movement allow a particular load to be applied to a given pavement area for a longer period of time resulting in greater damage [Yin et al. 2008]. For HMA pavements this behavior is sometimes evident at bus stops, intersection approaches and road sections where there are more traffic congestions. Greater thicknesses and/or higher qualities of pavement materials are therefore required for roads in urban areas and on uphill gradients because of relatively lower traveling speed.

\begin{tabular}{|l|c|c|c|}
\hline \multicolumn{4}{|c|}{ Speed limit (km/hr) } \\
\hline Country & Built up areas & Outside built up areas & Motor ways \\
\hline Belgium & 50 & $90-120$ & 120 \\
\hline Czech Republic & 50 & $90-130$ & 130 \\
\hline Germany & 50 & 100 & 130 \\
\hline Denmark & 50 & 80 & $110-130$ \\
\hline Italy & 50 & $90-110$ & $130-150$ \\
\hline Sweden & $30-50$ & $70-110$ & $90-110$ \\
\hline United Kingdom & 48 & $90-112$ & 112 \\
\hline
\end{tabular}

Table 2.1 Speed limit in some European countries [Capros et al. 2008]

Many of the existing mechanistic pavement response models do not include vehicle speed as an important factor; however, evidences showed that the magnitude of the calculated pavement strain response decreased with increased vehicle speed [Siddharthan et al. 2002]. Table 2.1 
above shows the speed limit in some European countries. However heavy vehicles operate below the specified speed limit and incur high stress-strain state in the pavement. The maximum compressive strain increases from $150 \times 10^{-6}$ to $300 \times 10^{-6}$ when the speed of the vehicle decreases from 90 to $20 \mathrm{kph}$ [Chen et al. 2004], implying that high compressive strain would result more rutting to occur when the traffic traveled at a lower speed. With increasing traffic speed, the portion of positive strain increases, thus sometimes moving to the tension region. Lower speeds primarily result in compressive strains, whereas higher speeds cause tensile and compressive strains alternately.

Current freeway designs have nearly reached the goal of allowing drivers to operate at high speeds in comfort and safety [1]. Nevertheless, precaution is required in case of rough surface, the dynamic impact that the pavement experience would be higher at high traveling speed.
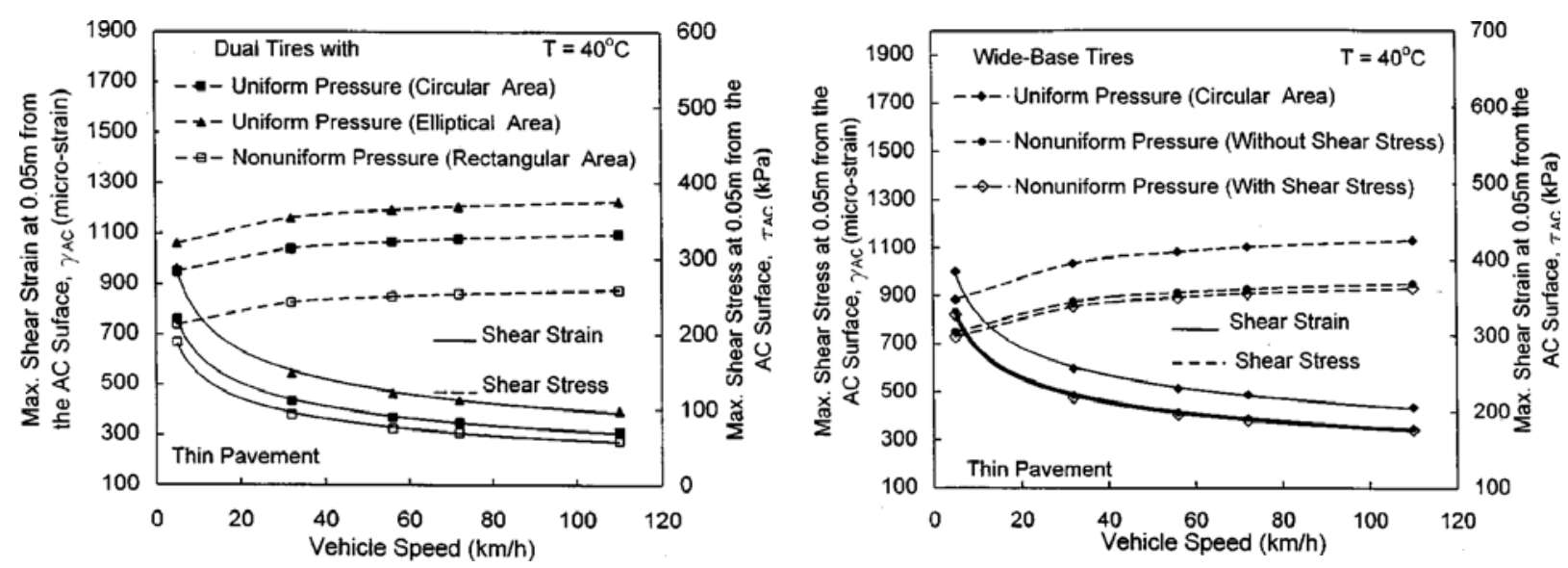

Figure 2.5 Variation of shear strain and shear stress with vehicle speed for dual and wide-base tires on thick pavement [Siddharthan et al. 2002]

\subsubsection{Traffic Load}

The primary function of the pavement is to transmit and distribute the wheel loads to the supporting subgrade. The total effect of traffic is related to the number and the size of the wheel loads. The simplest pavement structural model asserts that each individual load inflicts a certain amount of unrecoverable damage. This damage is cumulative over the service life of the pavement. When it reaches some maximum value the pavement is considered to have reached the end of its useful service life.

Traffic loads vary from light to heavy for passenger cars and loaded trucks, respectively. Small cars do not contribute significantly to the structural damage, particularly for paved roads even though their count is included in any regular traffic count survey. The design and evaluation of AC pavements are based on the number heavy vehicles and their loading characteristics.

Since pavement materials have non-linear response, doubling the load magnitude does not result in doubling the stress or strain. More importantly, doubling the load magnitude does not result in doubling the rate of pavement deterioration. In fact, increasing the load magnitude exponentially increases the rate of pavement deterioration.

Most pavement structural design standards require quantification of all expected loads a pavement encounters over its design life. This quantification is usually done in one of two ways: Equivalent single axle loads (ESALs) or Load spectra.

\section{Equivalent single axle loads (ESALs)}

Different pavement sections were subjected to various load levels and different load configurations (single or tandem axles). This is even more complex for actual pavement sections 
because each section is subjected to a distribution of loads and configurations. A possible solution to this is to use the cumulative number of equivalent single axle loads (ESALs). This approach converts wheel loads of various magnitudes and repetitions of "mixed traffic" to an equivalent number of "standard" or "equivalent" loads. It is used in pavement design methodologies to describe the relative amount of damage made to the pavement. The most common expression of pavement damage is the $80 \mathrm{kN}$ equivalent single axle load. Load equivalency factors (pavement damage factors) are used to describe the relative amount of damage for a specific axle loading and axle configuration in terms of the amount of damage on the pavement by some number of equivalent $80 \mathrm{kN}$ axle loads. Load equivalency factors are calibrated to specific pavement design procedures. They differ with different pavement design procedure, pavement type and other criteria, therefore, there are various approaches which can be used to convert a mixed stream of different classifications of vehicles, axle loads and configurations into an equivalent number of $80 \mathrm{kN}$ (or 10 ton axle load in case of Germany standards) single axle loads (ESAL's) and to sum these over the design period. The equivalency factor may take a wide range of values.

$$
E F=\left[\frac{L}{L s}\right]^{n}
$$

Where $E F$ - equivalency factor

$L s$ - Standard axle load (usually $80 \mathrm{kN}$ )

$L-$ any axle load

$n$ - power factor

For flexible pavements, values $n$ of 2-6 [Cebon 1993] have been cited in the literature. The 'fourth power law' is usually used to convert the estimated traffic during the service life into an equivalent number of standard wheel loads. The validity of the 'fourth power law' is, however, questionable, particularly for current axle loads and axle group configurations, tire sizes and pressures, road construction and traffic volumes: all of which are significantly different from the conditions of the road test.

The variety of wheel loads in the traffic stream and their cumulative applications are converted into a single number of ESAL applications that produce the same damage as the actual traffic loads using a load equivalency factor (LEF) concept [Archilla and Madanat, 2000]. This methodology still has some validity; however, it has several weaknesses when it comes to considering the impacts of higher tire pressures and new tire types and axle configurations.

\section{Load spectra}

This approach characterizes loads directly by number of axles, configuration and weight. It does not involve conversion to equivalent values. In essence, the load spectra approach uses the same traffic data that the ESAL approach uses only it does not convert the loads into ESALs - it maintains the data by axle configuration and weight [49]. This information can then be used with a series of mechanistic-empirical equations to develop a pavement structural design. Structural design calculations using load spectra are generally more complex than those using ESALs. Some key advantages of the load spectra approach are: it offers a hierarchical approach to traffic data input depending upon the users needs and resources and it already includes information on traffic distribution including directional, lane and temporal distribution (if needed) as well as traffic growth rates.

Weigh-in-motion (WIM) devices are designed to capture and record truck axle weights and gross vehicle weights as they drive over a sensor [49]. Gross vehicle and axle weight monitoring is useful in an array of applications including pavement design, monitoring and research, size and weight enforcement, and legislation and regulation works. The system uses Automated Vehicle 
Identification (AVI) to identify the vehicles; employs technologies that measure the dynamic tire forces of the moving vehicle, and then estimates the corresponding tire loads for a static vehicle.

\subsubsection{Contact Pressure}

Tire loads are the fundamental loads at the actual tire-pavement contact points. Most pavement analysts have assumed that the normal component of the contact pressure between tire and road surface is uniform, acts over a circular area and nominally equal to the inflation pressure.

$$
\text { Contact } \text { pressure }=\frac{\text { Load on wheel }}{\text { Contact area }}
$$

The interaction between vehicle and pavement is complex since pavement roughness excites the dynamic forces generated by vehicles, while these dynamic forces simultaneously increase the pavement roughness. Researches have been conducted to have better understanding of how the vehicle dynamic loads affect contact pressure within the tire imprint for different pavement roughness levels, vehicle types and speeds, tire types, and tire [Blab and Harvey 2002, Cebon 1993, Hua and White 2002, Park et al. 2005, Siddharthan et al. 2002, Simon 2007].

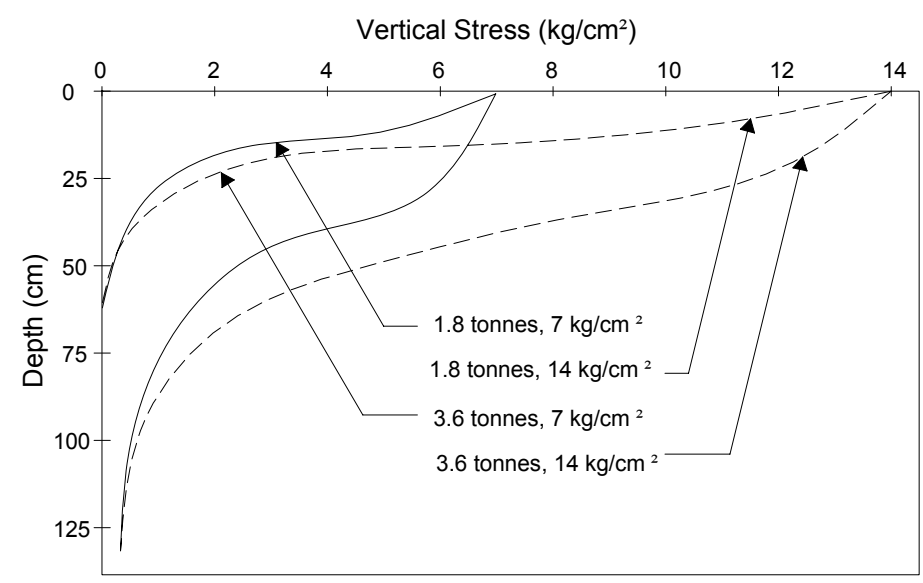

Figure 2.6 Vertical stress distribution with different magnitudes of contact pressure and wheel load

High tire inflation pressures, especially over-inflated wide-base single tires, greatly increase the fatigue damage to AC pavements. The effects of high contact pressures are most pronounced in the upper layers of a flexible pavement and influence the quality of material to be used. High magnitudes of tire pressure demands high quality of materials in the upper layer. The variation of the vertical stress with depth is given in Figure 2.6 above. At greater depth the effect of tire pressure diminishes and the total load exhibits a considerable influence on the magnitude of the vertical stress. With constant tire pressure, the total load governs the stress on the top of the subgrade layer.

It has been also noted that the contact pressures under each tire in a pair can be quite different due to a number of factors, including differential inflation pressures or temperatures, tread wear, axle bending or transverse road roughness. It is indicated that a wide-base single tire is only 1.5 times more damaging than an unevenly inflated dual pair with $500 \mathrm{kpa}$ in one tire and $1000 \mathrm{kpa}$ in the other [Cebon 1993].

Considerable evidences [Cebon 1993, Park et al. 2005, Siddharthan et al. 2002] suggested that the pressure have been observed to increase around the edges of the contact area, particularly in the 'shoulder' areas at either side, due to the stiffness of the side walls and bending of the tread band. Under normal inflation and loading conditions, the maximum shoulder pressure has been typically observed to be twice [Cebon 1993] the inflation pressure, although the contact pressure 
distribution has been found to be more uniform for higher inflation pressures and/or lower vertical loads.

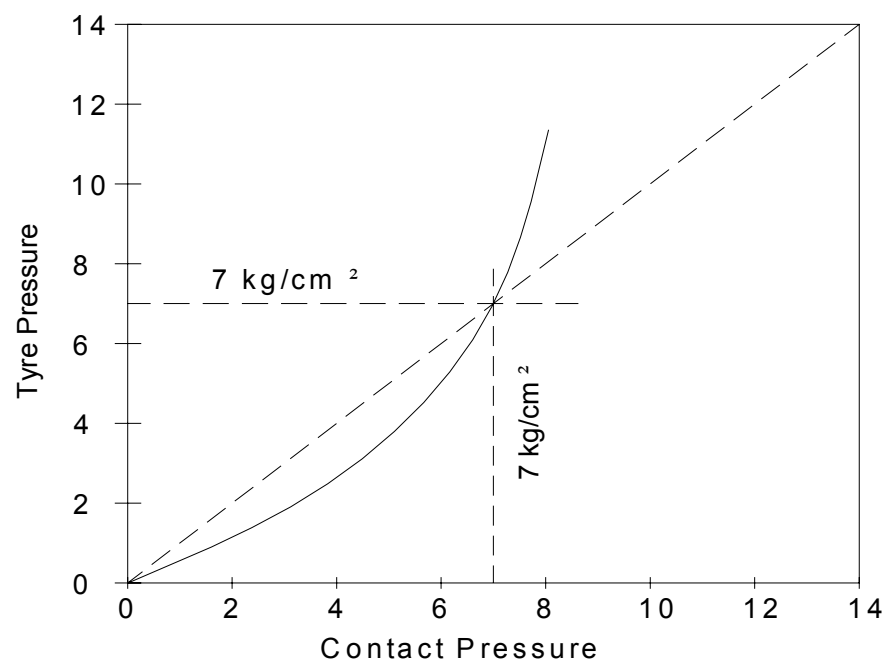

Figure 2.7 Relationship between tire and contact pressure

The contact area is also found to decrease with increased inflation pressure and to increase with total load. It is important to note that even in straight-line-steady-speed motion; the contact pressure distribution between tires and road surfaces contains significant lateral and longitudinal shear tractions as well as the vertical pressure due to the differences in the stiffness of the sidewalls, tread-band and tread. On the basis of asphalt pavement strain measurements, it has been reported in literature [Cebon 1993] that an increase in tire pressure would increase fatigue damage. Elevated tire inflation pressure greatly increases the fatigue damage of flexible pavements. Over inflation of conventional dual tires by $170 \mathrm{kpa}$ nearly doubles flexible pavement fatigue. Similarly over-inflation of wide-base single tires is especially critical, increasing fatigue (damage) by a factor of four. Currently there is a trend that average inflation pressures have increased from $550 \mathrm{kpa}$ to $690-760 \mathrm{kpa}$ [Cebon 1993]. There is concern in the pavement engineering community that these changes in operating patterns may increase pavement damage, particularly fatigue and rutting.

\subsubsection{Effect of Heavy Vehicles and Trend of Growth Rate}

Pavement design is basically concerned with protecting the subgrade and the various courses within the pavement structure, from excessive stresses and strains imposed by commercial vehicles and that it is the wheel loads of these heavy vehicles that are the primary contributors to pavement distress [Gillespie et al. 1993]. Deflections due to truck loads produce stresses and strains that lead to permanent deformation in the surface and subsequent layers of the pavement system. As truck volume increases, cyclic strain at the bottom of the asphalt layer also leads to fatigue cracking.

Besides being heavier, trucks are generally slower and occupy more roadway space. Consequently, trucks have a greater effect on highway traffic design than do passenger vehicles. Apart from various environmental effects, road deterioration is predominantly caused by forces applied by repeated truck loads. The amount of damage applied depends on gross and axle weights, number and location of axles, dynamic impact of loads, tire properties, etc.

The growth of truck traffic has resulted in an increase in the number of loads applied, while at the same time axle loads and tire pressures have also increased. New configurations, suspensions, tires and higher tire pressures have changed the characteristics of the loads applied to the pavement surface over the past several years. 
Heavy vehicles cause a disproportionately high percentage of the damage to the road infrastructure, as there is an exponential relationship between axle load and road damage. Pavements are subjected to a cumulative load (expressed as the equivalent number of standard axles) related to heavy vehicles axle loadings (HV), usually $80 \mathrm{kN}, 100 \mathrm{kN}$ or $130 \mathrm{kN}$, depending on the country.

An increase in global economy and countries' economy expansion, along with transport reliance on trucks to move freight lead to an increasing number of existing and new types of trucks used to move more goods on existing roads. Road transport services account for $1.6 \%$ of the GDP and give jobs to 4.5 million people in the EU [48].

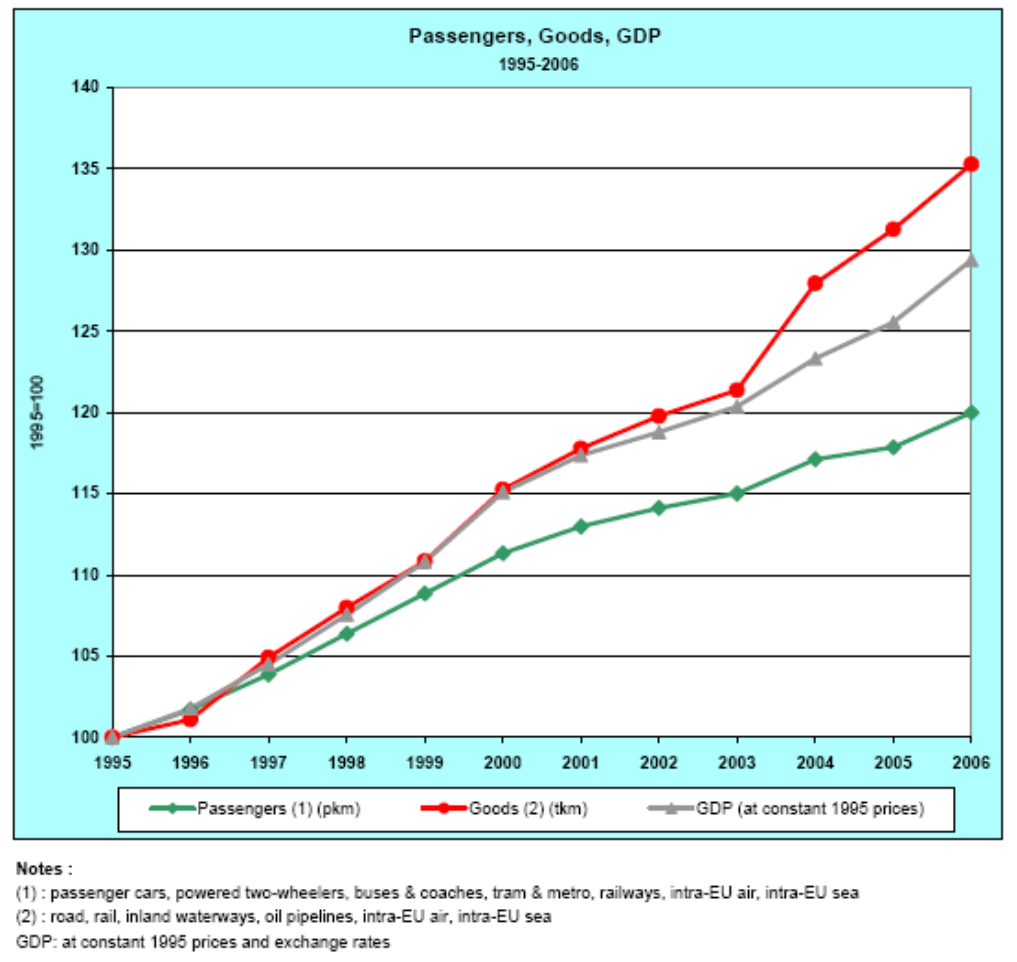

Figure 2.8 Traffic growth in EU27 [Capros et al. 2008]

The whole economy and society depends heavily on efficient road transport, $44 \%$ of the goods are moved by trucks and $85 \%$ of the persons by cars, buses or coaches. Growth rate of trucks

\begin{tabular}{|l|c|c|c|c|}
\hline $\begin{array}{l}\text { GDP at } 1995 \text { prices and } \\
\text { exchange rates }\end{array}$ & $\begin{array}{c}1995-2006 \\
\text { p.a. }\end{array}$ & $2.4 \%$ & $\mathbf{2 0 0 5 - 2 0 0 6}$ & $3.0 \%$ \\
\hline $\begin{array}{l}\text { Passenger transport } \\
\text { pkm }\end{array}$ & $\begin{array}{c}1995-2006 \\
\text { p.a. }\end{array}$ & $1.7 \%$ & $\mathbf{2 0 0 5 - 2 0 0 6}$ & $1.8 \%$ \\
\hline $\begin{array}{l}\text { Freight transport } \\
\text { tkm }\end{array}$ & $\begin{array}{c}1995-2006 \\
\text { p.a. }\end{array}$ & $2.8 \%$ & $\mathbf{2 0 0 5 - 2 0 0 6}$ & $3.1 \%$ \\
\hline
\end{tabular}

Table 2.2 Annual growth rate in EU-27 [Capros et al. 2008]

varies depending on the country and the economic development of the area. By the year 2000 the total number of vehicles on European roads [33] were estimated to increase by $20 \%$ to over 27 million with heavy vehicles (HV) growing by 15\% over the same period. Approximately 90\% of all the UK's inland freight was moved by road. In France and Germany the number of HV has grown by $25 \%$ in 1999-2000 and it was anticipated that growth would continue into the next 
century. The demand [Capros et al. 2008] for the land transport modes road, rail, inland waterways and pipelines in the EU-27 added up to 2376 billion ton-km in 2005 and 2595 billion ton-km in 2006. Road accounted for $72.6 \%$ of this total, rail for $16.5 \%$, inland waterways for $5.4 \%$ and pipelines for the remaining 5.5\%. The growth rate of traffic in Ethiopia has been estimated to be $4.8-6 \%$ based on the review of various traffic studies conducted in Ethiopia over the past several years [36].

\section{Axle and Tire Configurations}

Highway traffic consists of an array of vehicles with various weights and axle configurations. Although it is not too difficult to determine the wheel and axle loads for an individual vehicle, it becomes quite complicated to determine the number and types of wheel/axle loads that a particular pavement will be subject to over its entire design life. But the wheel load configurations are important to know the way in which the loads of a given vehicle are applied on the pavement surface.

\begin{tabular}{|c|c|c|c|}
\hline 4 & 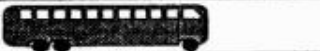 & BUSES & $2 \& 3$ \\
\hline 5 & & 2-AXLE, SINGLE UNIT & 2 \\
\hline 6 & & 3-AXLE, SINGLE UNIT & 3 \\
\hline 7 & & A-AXLE, SINGLE UNIT & 4 \\
\hline 8 . & & $\begin{array}{l}\text { 2-AXLE TRACTOR, } \\
\text { 1-AXLE TRLR(2S1) } \\
\text { 2-AXLE TRACTOR, } \\
\text { 2-AXLE TRLR(2S2) } \\
\text { 3-AXLE TRACTOR, } \\
\text { 1-AXLE TRLR(3S1) }\end{array}$ & $\begin{array}{l}3 \\
4 \\
4\end{array}$ \\
\hline 9 & & $\begin{array}{l}\text { 3-AXLE TRACTOR, } \\
\text { 2-AXLE TRLR(3S2) } \\
\text { 3-AXLE TRUCK. } \\
\text { W/2-AXLE TRLR }\end{array}$ & 5 \\
\hline 10 & & $\begin{array}{l}\text { TRACTOR W/ SINGLE } \\
\text { TRLR }\end{array}$ & $6 \& 7$ \\
\hline 11 & & $\begin{array}{l}\text { S-AXLE MULTI- } \\
\text { TRLR }\end{array}$ & 5 \\
\hline 12 & & $\begin{array}{l}\text { 6-AXLE MULTI- } \\
\text { TRLR }\end{array}$ & 6 \\
\hline 13 & ANY 7 OR MORE AXLE & & 7 or more \\
\hline
\end{tabular}

Figure 2.9 Typical heavy vehicle type and axle configuration [53]

The number and spacing of axles are important factors for effectively transmitting the load onto the pavement surface. An increase in the number of axles provides additional contact points, and thus reduces the load at each point. Axle spacing does affect pavement responses, such as deflections, stresses, and strains. Axle configurations used today are single, tandem tridem axles or under some circumstances multiple axles.

Analysis of many pavement structures has revealed that pavement rutting and fatigue damage are not only a function of gross vehicle weight but also a function of axle weight. The axle weight and configuration govern the magnitude of surface deflections, stresses and strains in asphalt concrete pavements. Most researchers agree that damage is mostly influenced by the load, number, type, and spacing of a vehicle's axles. 
To maintain the maximum wheel load with in the specified limit and to carry greater load manufacturers are providing wide-base-tire for road users offering a more economical and effective transport of goods, but such vehicles exert on the other hand a more aggressive action on the pavement structure. Wide-base single tires ('super singles') are replacing dual tires in Europe, particularly on triaxle trailer group suspensions. A tandem axle with wide-base tires is clearly more damaging than a tandem axle with standard dual tires. In addition, tandem axles are superior to single axles based on the vertical stresses in both the subbase and subgrade. On the basis of asphalt strain measurements, it is reported [Gillespie et al. 1993] that wide-base single tires are likely to cause shallower rate depth than conventional single tire for the same loading, however cause a wider rut because of it greater contact width. When rutting is considered as a criteria under an individual tire, single and wide-base single tire have been worse than dual tires.

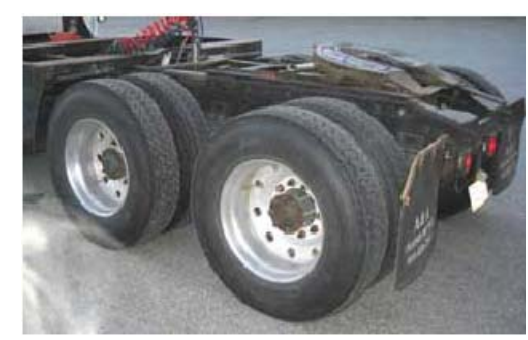

a. Tandem wheel assembly

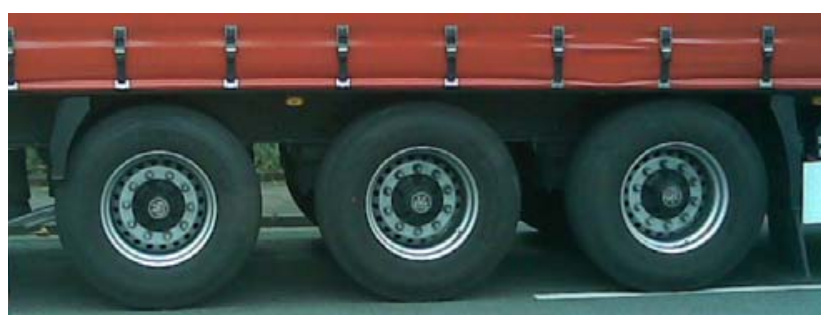

b. Tridem wheel assembly with wide-base tire

Figure 2.10 Heavy vehicle wheel configuration

While the tire contact pressure and area is of vital concern in pavement performance, the number of contact points per vehicle and their spacing is also critical. As tire loads get closer together their influence areas on the pavement begin to overlap, at which point the design characteristic of concern is no longer the single isolated tire load but rather the combined effect of all the interacting tire loads. Therefore, axle and tire arrangements are quite important to address the various responses.

\subsubsection{Traffic Wander}

On the other hand the distribution of vehicles traveling path in the transverse direction plays significant role in pavement performance. Current models relating traffic level to pavement distresses hardly incorporate the effect of traffic wander, even though pavements are subjected to loads whose repetitions are distributed over an area several times the width of a vehicle tire.

At the beginning of the service life the evenness of the pavement may give the drivers some sort of psychological freedom to maneuver their vehicles with in the available lane space. Here the wander effect plays a constructive role in minimizing the rut formation. After some period of time the gradual appearance of rutting along the wheel track would rather compel drivers to adhere the wheel track which ultimately accelerate the rut formation. 

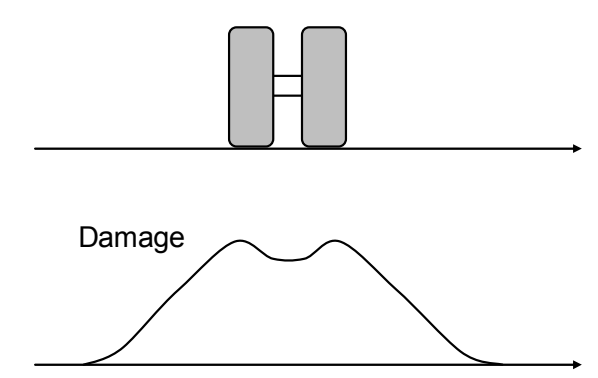

Wander Normal Distribution

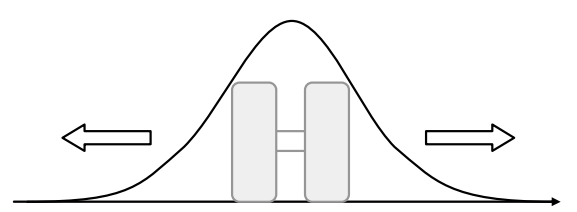

Figure 2.11 Normally distributed lateral wander of traffic wheel

The wheel path distribution in the transverse direction of a pavement has its own contribution to the performance of the pavement [White et al. 2002, Hua and White 2002]. Several factors affect wheel path distribution, including roadway geometry, lateral clearances, traffic conditions, roadway characteristics, weather conditions and vehicle type.

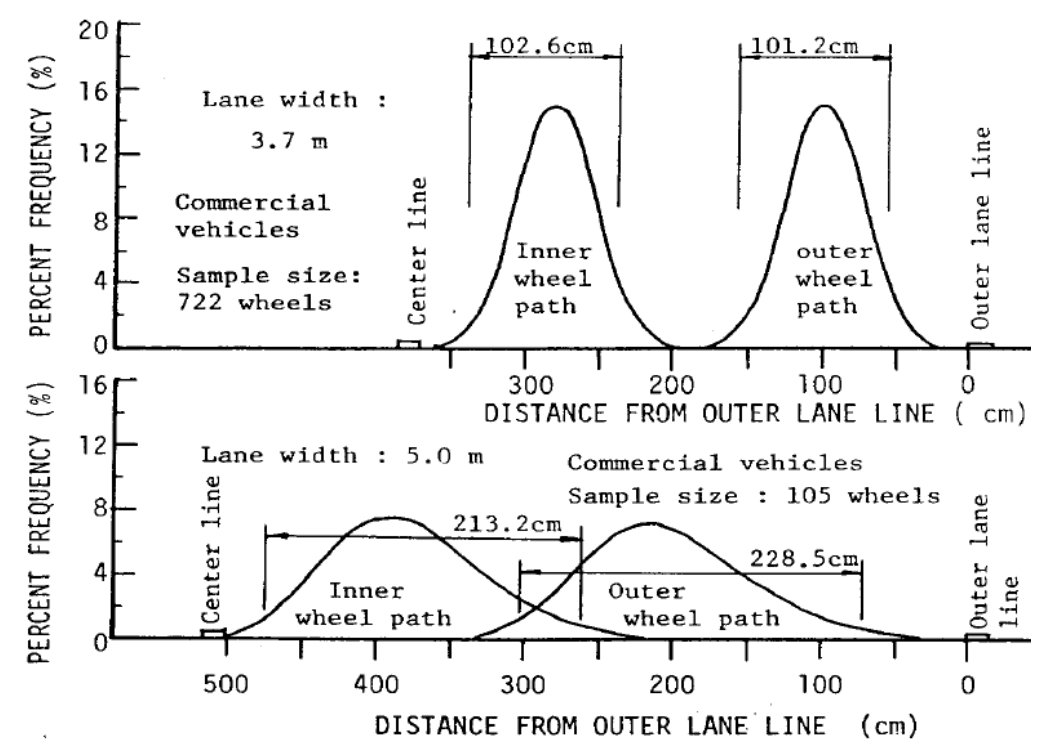

Figure 2.12 Influence of lane width on wheel path distribution [White et al. 2002]

It has been indicated that the width of the traffic lane was the most significant parameter in determining the extent to which the wheel paths were distributed over the road surface, with wider distributions observed for wider lanes. They also observed that the wheel path distributions on four-lane pavements were slightly wider than on two-lane pavements.

Lateral wander of traffic influences the number of axle load applications over a point for predicting distress and performance. This parameter affects prediction of fatigue and permanent deformation within the pavement system. An increase in wander will result in more fatigue life and less permanent deformation within the pavement system. It is usually not practical to assess the exact distribution of wander; however, a good approximation is to assume that the wander is normally distributed. 


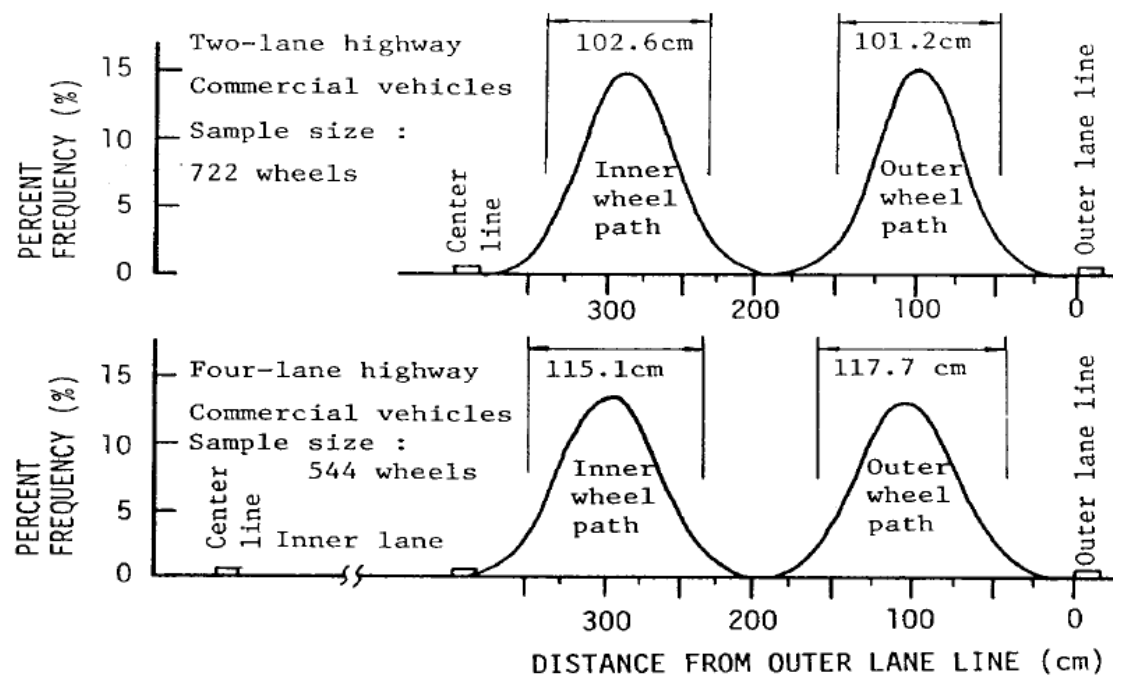

Figure 2.13 Influence of number of lanes on wheel path distribution [White et al. 2002]

\subsection{Materials and Structural Properties}

Better understanding of materials behaviors and structural responses of asphalt concrete in combination with load-stress-strain computation tools, allows for optimization of asphalt pavement thickness and material choice. The structural design of a road pavement is a balance of a number of requirements based on quality of construction materials, stability and durability of component layers and construction techniques. Materials are the integral part of a pavement structure which requires a thorough investigation and assessment for their suitability and durability for construction. Failure of a material and structural properties to fulfill strength and other quality requirement set by design standards would have serious impact on pavement performance, therefore, a due consideration and proper evaluation is unquestionable.

\subsubsection{Materials Characteristics}

The selection of pavement materials is a critical element in the design, construction and maintenance of pavements if performance is to be optimized. Construction materials have their own characteristics suited to a specific design and construction use. The two basic materials in AC pavement are the bitumen and the aggregate which have their own distinct behaviors.

\section{Binder}

Binder (sometimes termed as bitumen/asphalt) is a general description for the adhesive or glue used in asphalt pavements, either petroleum derived or naturally occurring material [Lavin 2003]. The asphalt binder is what gives an asphalt pavement its flexibility, binds the aggregate together, and gives waterproofing properties to the pavement.

Even though the binder content is a key mixture design parameter, the binder grade plays a significant roll on the performance of pavement. Selecting a binder grade is essential in insuring that the asphalt will not experience significant levels of distress at the prevailing climatic condition. Asphalt binders are visco-elastic materials in nature whose resistances to deformation under load are sensitive to loading time and temperature. Less viscous asphalts make the mixture less stiff and therefore more susceptible to irrecoverable deformations, i.e., rutting. On the other hand, if asphalt is too hard, it would be brittle at low temperatures ultimately leading to cracking under loading. 


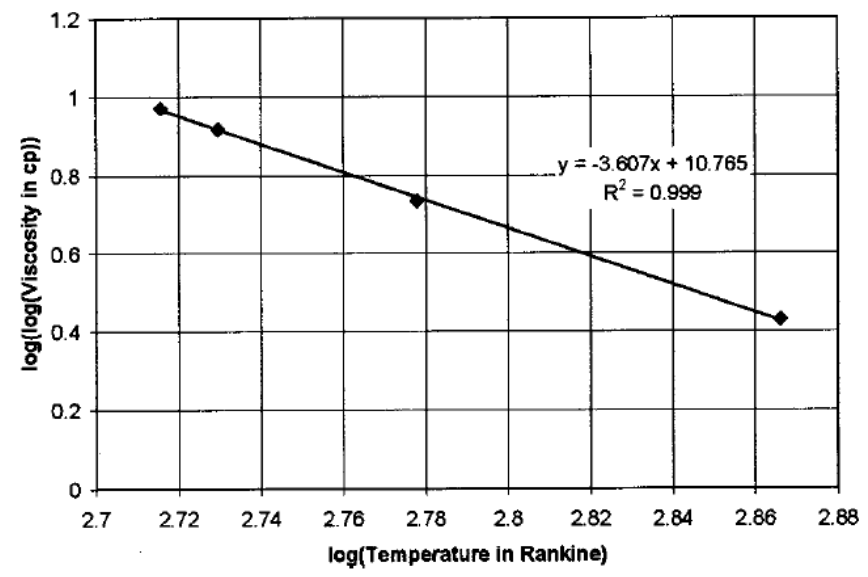

Figure 2.14 Plot and least-squares fit for example binder [Rasmussen et al. 2002]

Recent studies have shown that a binder grade is selected based on the pavement temperature expected in the AC layers [Shenoy 2001, Yavuzturk et al. 2005]. The demand for asphalt binder with reduced temperature susceptibility is increased. Modification of the binder with polymer and other modifying agents are most commonly used mechanisms employed for improving its thermal susceptibility [Bahia et al. 2001]. For example the high modulus asphalt binder (HAMB) [Lee et al. 2007] was developed for high modulus asphalt mix (HMAM) applicable to the asphalt base layer for long life asphalt pavements. HMAB was developed using the high boiling point petroleum and polymer. The high boiling point petroleum added to the conventional asphalt binder increased the binder stiffness.

\section{Aggregates}

Aggregates are the key materials used in the construction sector and the largest portion of an asphalt pavement. Aggregates are generally derived from stone minerals and sometimes further mechanically processed to suite for specific applications. Synthetic aggregates, most commonly blast furnace slag from the steel industry, slate wastes and ashes, are also used in the construction of asphalt pavements [O'Flaherty 2007].

Mineral aggregate (predominantly of coarse aggregate) constitutes approximately 90-95\% of hot-mix asphalt (HMA) by weight. Research has shown that aggregate characteristics such as particle size, shape, and texture influence the performance and serviceability of hot-mix asphalt pavement [Chen et al. 2005]. Aggregate shape is one of the important properties that are considered in the mix design of asphalt pavements to avoid premature pavement failure. Flat and elongated particles tend to break during mixing, compaction and under traffic loading. It has been found [Prowell et al. 2005, Chen et al. 2005] a direct correlation between the rutting potential of HMA mixtures and the shape and texture of coarse aggregate particles. Some mixes with flaky aggregates have been found to exhibit higher fatigue life than mixes with non flaky aggregates. Mixtures made from angular aggregates (obtained by crushing) deform to a minor extent and are more stable than mixtures having the same composition and grading but made from rounded aggregates (river gravel).

The percentage of crushed coarse particles has a significant effect on laboratory permanent deformation properties. As the percentage of crushed coarse particles decreased, the rutting potential of the HMA mixtures increased. It is emphasized in literature [Chen et al. 2005] that cubical, rough-textured aggregates have better interlocking mechanisms; reduce the potential for rutting and more resistant to the shearing action of traffic than rounded and smooth-textured aggregates.

Some researchers [Sousa et al. 1991] indicated that dense aggregate properties and gradations are desirable to mitigate the potential effects of rutting of asphalt concrete pavement. When properly compacted, mixtures with dense or continuous aggregate gradations have fewer voids 
and more contact points between particles than open or gap-graded mixtures. For example, a gap-graded mixture exhibits more deformation than a continuously graded mixture due to less aggregate interlock in the gap-graded mixture. Aggregate interlock becomes more important at higher temperatures; gap-graded mixtures may be even more susceptible to rutting at higher temperatures. As well as, the use of larger maximum aggregate size (about two-thirds of layer thickness) would be beneficial in reducing the rutting propensity of mixtures subjected to high tire pressures.

For better rutting resistance the surface texture of the aggregate plays an extremely important role. Particularly in thicker asphalt-bound layers and hot climates, a rough surface texture is required.

\section{Asphalt Mixtures}

A vital component in the process of constructing an asphalt pavement is the design of the asphalt mixture. "Asphalt mixtures are different from most engineering materials in that the highest strength mixture design is not necessarily mean the best choice for the particular asphalt pavement application" [Lavin 2003]. The loading, desired surface texture, environmental conditions and other factors are all considered in the designing of an asphalt mixture.

For the past many years, asphalt concrete mixtures have been characterized using conventional laboratory test methods in order to determine their properties and study the effect of rate of loading, temperature and materials properties on mix behavior.

Regardless of the mixture type, mix design process involves determining the type and proportion of materials. The stability and durability of the asphalt mixture are the two primary characteristics that are determined at the mix design stage.

The response and behaviors of asphalt mixtures are heavily influenced by temperature, time rate of load, method of mixture, and the mixing process. Dense graded asphalt mixtures or hot mix asphalt (HMA) is the most common material used for asphalt pavements. A dense graded mixture with greater aggregate size and large amount of coarse aggregate are typically used as a base course, where significant strength might be required while being protected from the effects of the environment.

Open graded mixtures/open graded asphalt friction courses/ are increasingly being specified as high-speed wearing courses due to their ability to drain water quickly and provide skid resistance for vehicles.

Stone mastic asphalt (SMA) was developed in Germany (known as "Splittmastixasphalt.") in the late 1960s as an effort to resist the detrimental effects of tires equipped with winter driving studs [Lavin 2003, Lane et al. 2008]. However, SMA pavements proved to be very durable, rut resistant and the most common road surfacing material in use in Germany today. SMA mixture consists of coarse aggregates and mastic of fine aggregate, mineral filler, and asphalt binder. It has very high strength due to its stone skeleton that can provide a very stable and durable rutresistant wearing course for highway applications. The increased asphalt cement content makes it less susceptible to low temperature cracking, provides crack healing properties, fatigue resistance, good aging properties and improved durability. The macrotexture of the SMA mixes has been found to provide improved surface drainage in comparison with dense-graded mixes. SMA mixes have low air voids that can protect the underlying mixes from water infiltration.

The binder content and the air void affect the mixture's ability to resist permanent deformation. The increase in asphalt content is equivalent to the introduction of lubricants between aggregate particles otherwise separated by a very tight network of air voids. This phenomenon causes the mixture with the higher asphalt content to be more susceptible to permanent deformation. To preclude problems of instability and, therefore, permanent deformation, it is recommended an absolute minimum (of 3-4 percent) air voids in the mix. 


\subsubsection{Structural Characteristics}

The thickness of the pavement layer is the most significant part of the structural integrity of the asphalt pavement. Variables evaluated when determining thickness of asphalt pavement are subgrade, subbase and AC material properties, traffic loading and environmental factors. Thickness determination of a pavement is a structural evaluation process, ensuring that the traffic loads are so distributed that the stresses and strains developed at all levels in the pavement structure and in the subgrade are within the capabilities of the materials used at those levels. It involves the selection of materials for the different pavement layers and the calculation of the required thickness. The traffic or load carrying ability of an asphalt pavement is a function of both the thickness of the material and its stiffness.

The structural failure of a pavement is associated with the strength characteristics of the constituent materials and the thickness of each layer. Major failure types are surface rutting and crack formation either at the top surface or bottom layers. The required thickness of each layer of the flexible pavement varies widely depending on the materials used, magnitude and number of repetitions traffic load, environmental conditions, and the desired service life of the pavement. These factors are generally considered in the design process so that the pavement would last for the required designed life without excessive distresses.

It can be seen that the load is distributed on a small area at the surface. As the depth increases, with same load, the stress developed is distributed over larger area; therefore, the highest stress occurs at the surface and it decreases as the depth increases. Thus, highest quality materials are required at the top of the pavement and as the depth increases lower quality materials can be used. A typical flexible (or asphalt) pavement consists of surface (wearing and binder), base and subbase courses built over compacted subgrade (natural soil).

The primary function of surface course is to provide a safe, smooth, stable riding carriageway for traffic and to contribute to the structural stability of the pavement and protect it from the natural elements.

The base and binder courses are the main structural component of an asphalt pavement. Both courses transmit and distribute the traffic loading so that the strength capacities of the weaker sub-base and subgrade are not overstressed. They are designed to be dense and highly stable to resist permanent deformation and fatigue cracking caused by repeated traffic loading and distresses induced by temperature fluctuations throughout the structure during the service life.

A subbase layer further distributes the induced stress to the weaker subgrade layer. Whilst the subbase material is of a lesser quality than the road base material, it has to be able to resist the stresses transmitted from the superstructure and at the same time be stronger than the subgrade soil. When the subgrade soil is weak or if there is frost action, a capping or frost protection layer may be provided above the subgrade for better bearing capacity.

\subsubsection{Innovative Materials}

A rapid growth in heavy traffic demands better performance road and hence requires better construction materials. The utilization of new materials type and modification of materials characteristics are under steady state to improve the performance and durability of asphalt concrete pavement.

Reports indicated that geomaterials has been laid between the base, subbase or subgrade material increased strength and enhance the bearing capacity of the substructure. Various research works [Ling and Liu 2001, Saad et al. 2006] are under progress over the utilization of geosynthetic materials that can be laid between structural layers to intercept the formation and propagation of cracks. The primary purpose of incorporating the use of geosynthetics in the pavement design process is to reduce reflective cracking in HMA overlays and to resist moisture intrusion into the underlying pavement structure. Some results [Ling and Liu 2001] indicated 
that the geosynthetic reinforcement contributed to an improvement in the stiffness and strength of asphalt pavement. The settlement over the loading area of reinforced pavement was reduced when compared with unreinforced pavement. Analysis of the effects of geosynthetic reinforcement revealed [Saad et al. 2006] the highest reduction of the fatigue strain when the material is placed at the bottom of $\mathrm{AC}$ and the maximum reduction in the value of rutting strain with in the base course. A geogrid reinforcement placed at the surface course/base course interface increases the rutting resistance of an asphalt pavement hence would be beneficial in increasing the service life of the asphalt pavement. Geogrid reinforcement provided a more uniform load distribution and a deduction in the rut depth both at the surface and base courses.
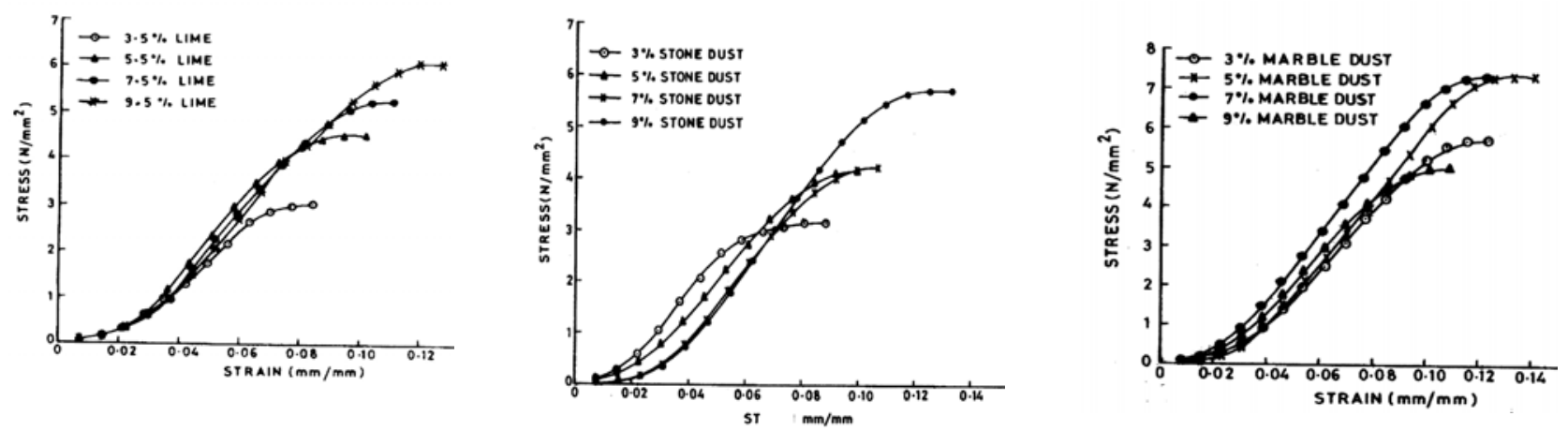

Figure 2.15 Effect of filler on compressive strength of asphalt concrete mixtures [Chandra et al. 2002]

Fillers are added to paving mixtures to impart greater stability and strength. At higher field temperatures, where the deformation behavior of asphalt pavement becomes critical, the highest possible viscosity of the filler-bitumen mastic is desirable as this will have a favorable influence up on the deformation resistance of the pavement. Different types and quantities of fillers are recommended to bear strength and influence the air void content and flow values of the hot mix asphalt [Chandra et al. 2002].

To improve the service life of pavements, innovative materials have been used in recent days to produce asphalt concrete layers that can better withstand distress phenomena. Following the development of hard grade paving asphalts (i.e. having penetration of less than 25/10 mm at 25 0C), high modulus asphalt concrete (HMAC) began to be used in France [Corté 2001]. These materials had a better mechanical behavior than traditional asphalt concretes relative to the distress mechanisms often considered in pavement design (cracking and permanent deformation). HMAC was first used to reinforce old pavements. It was subsequently used in the base layers of new pavements to obtain economic benefits by reducing thickness, especially where local aggregates had a low crushing index, or when traffic was intense, slow or channeled. HMAC were introduced into Portugal by the road administration who laid the mixture at a very busy access to a major bridge in Lisbon. It was the first attempt to use a stronger asphalt concrete to solve support capacity problems. The technique was repeated in 1996 and 1997 with similar objectives, to gain experience in HMAC production, paving operations and service behaviors.

Different modifiers are added to bitumen prior to mixing with aggregates to improve its inherent properties [Bahia et al. 2001, Centeno et al. 2008, Lee et al. 2007, Polacco et al. 2006]. The development of modified asphalt materials to improve the overall performance of pavements has been the focus of several research efforts made over the past few decades. The use of polymer modified asphalts in road pavement has been continually increasing worldwide. Several researches have been under progress to improve rutting performance by using modifiers which increase the viscosity of the asphalt binder at high temperatures without adverse effect at low temperatures. Relatively small quantity of polymer can significantly enhance performance and durability. Besides reducing deterioration and maintenance cost, polymer modified asphalt concrete improve resistance to abrasion as well as oxidation and aging [Polacco et al. 2006]. 
The performance improvement is directly associated with the influence of the polymer overall rheological properties.

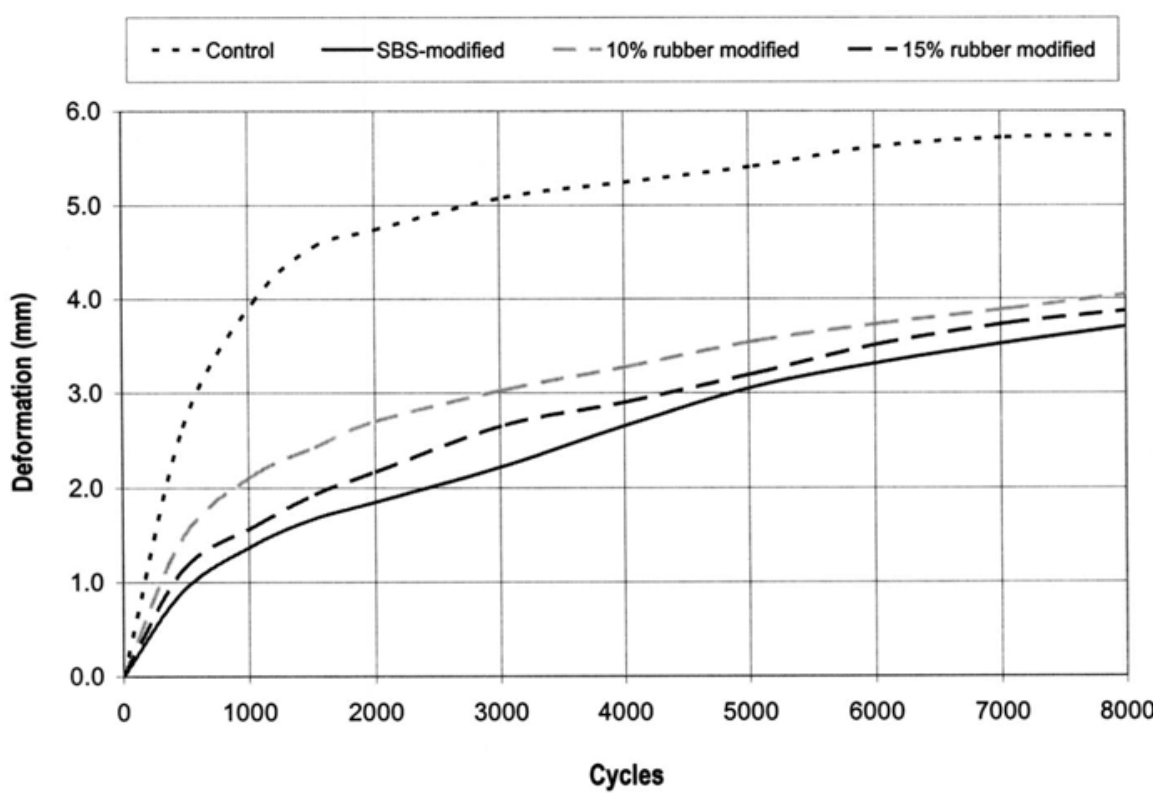

Figure 2.16 APA test results for four mixtures with $4 \pm 0.5 \%$ air void contents [Lee et al. 2007]

Epoxy Asphalt has been used for many years as a road surface on stiff bridge decking. It is a kind of high strength and flexible material by adding thermosetting epoxide resin and solidified agent into asphalt. Epoxy asphalt mix was found to be less susceptible to moisture damage, lower potential for permanent deformation and improved fatigue behavior, performed excellently at low temperature. Use of this material in ordinary pavement construction has been getting more attention found a premium material that outperforms conventional binders. It is a material with high stiffness that can be applied in thin surface layers. Acid-based Epoxy Asphalt [Wei et al. 2003] mixtures were found to have greatly improved performance compared to conventional mixtures most heavily trafficked road situations. The extensive testing undertaken had indicated that Epoxy Asphalt should have produced a durable, long lasting material suitable for use on heavily trafficked roads [73]. It was confirmed that epoxy asphalt was a premium material that outperformed conventional binders on the important indicators of potential long service life.

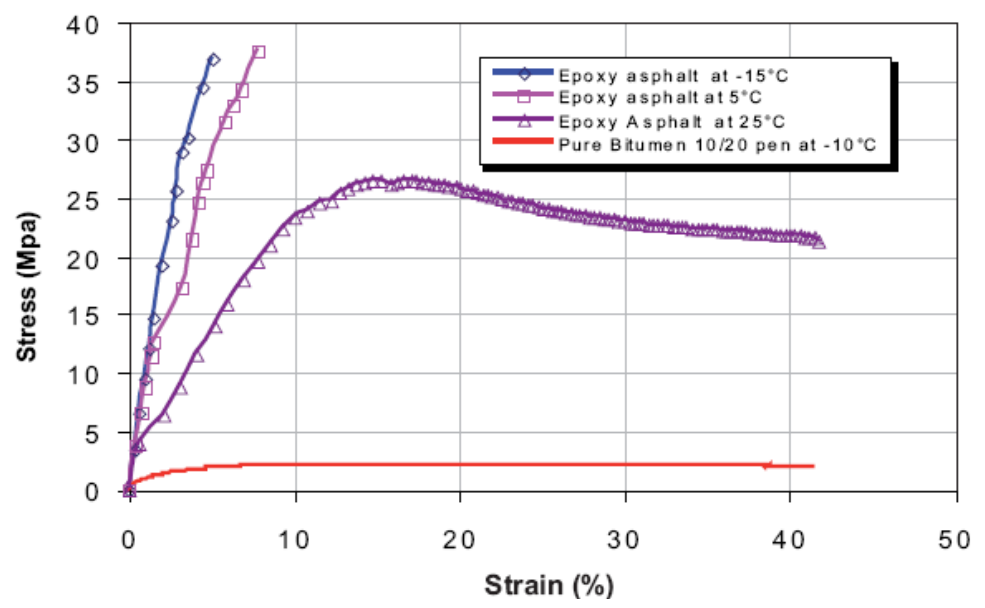

Figure 2.17 Stress strain curves for epoxy asphalt and hard bitumen at different temperatures [73] 


\section{Modes of Failure}

Degradations of road are caused by several factors and revealed as longitudinal and transverse unevenness of the road surface and the occurrence of different types of cracks caused by traffic loading, temperature cycling and frost heave at low temperature. If sufficiently high, the loading results in a loss of rigidity of the material and can, by accumulation in the long term, lead to failure. Therefore the repetitive traffic loading that the road experiences during its service life combined with environmental factors causes deformation, instability and other forms of deterioration which ultimately degrade the serviceability and durability of pavement structures.

Modes of deformation and the degree of susceptibility of the pavement to various deteriorating factors also depend on the type of pavement and materials characteristics. Temperature and seasonal climatic variations have a great impact on the different moduli of the pavement structures which gradually degrade with time.

The environment and the type of service the asphalt pavement is in, usually determines the type of distresses it may exhibit. High traffic, expressway, or motorway type asphalt pavements can undergo a type of distress known as rutting. A pavement can exhibit one or more distresses in various severity levels. Distresses can range from surface imperfections to distresses that lead to structural failure of the pavement. Most pavement distresses, if identified early, can be repaired and not have any significant effect on the service life of the pavement.

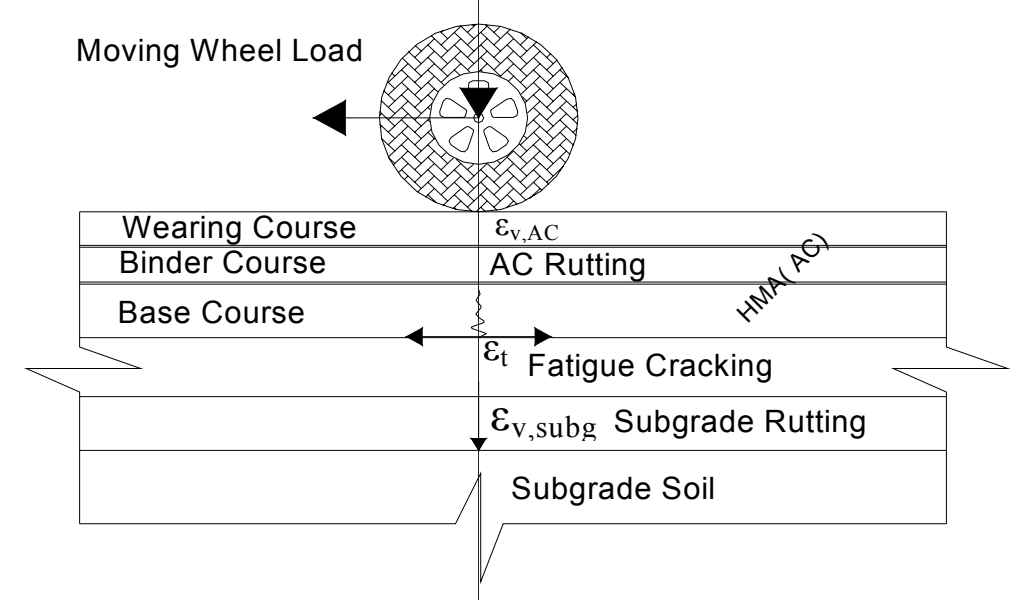

Figure 3.1 Mechanism of pavement structural failures

The identification of the various types of pavement distresses allows the pavement maintenance personnel to determine what type of remedial action is necessary. Therefore early detection and repair of defects in the pavement will prevent minor distresses from developing into a pavement failure. The most important road damage mechanisms are generally categorized as:

(i) Permanent deformation (longitudinal rutting);

(ii) Fatigue cracking;

(iii) Reduced skid resistance;

(iv) Low temperature cracking;

(v) Reflection cracking;

These distresses are ultimately accountable to the reduction of remaining life of pavements and increase of the rehabilitation and maintenance cost. Each failure mechanism is affected by many factors including the roadway design and construction methods, the material properties of each constituent layer, the traffic loading and the environmental conditions throughout the service life. 


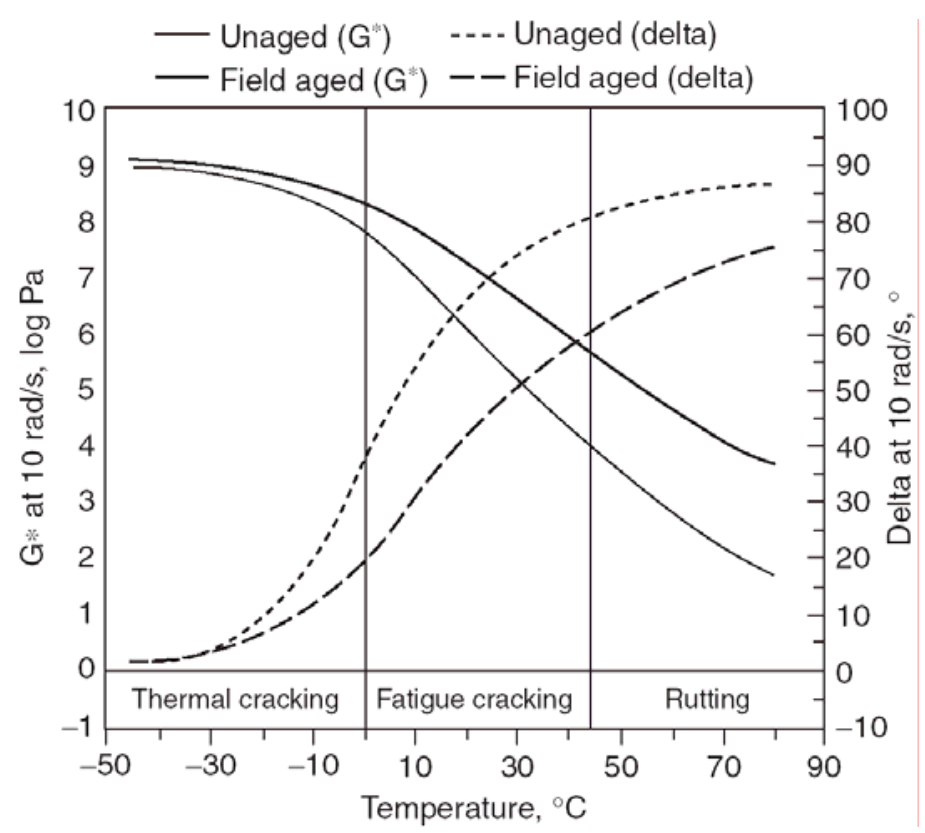

Figure 3.2 Effect of temperature on shear parameters [Bahia 2009]

\subsection{Rutting Failure}

It is widely agreed that rutting, a term commonly used to describe permanent deformation gradually develops along the wheel path as the number of load repetitions increases and accompanied by upheavals to the side. It represents an accumulation of small amounts of unrecoverable deformation that occurs each time a vehicle load is applied. The accumulation of permanent strain is considered as the most severe distressing mechanism with respect to pavement damage. It can be initiated in any layer of the structure, making it more difficult to predict it. Total unrecoverable deformation at the surface is the accumulation of the permanent deformation in all of the asphalt concrete and other structural layers in the pavement system. It principally occurs due to repetitive shear deformation under a variety of traffic loading. Loading type and magnitude, materials and structural characteristics, traffic flow and their behaviors and temperature are among the major contributors to rutting.

Rutting can be caused basically by mechanical deformation, consolidation of asphalt layer or displacement of the bituminous mixture due to lose of mixture stability. The stability strongly depends on grading and aggregate characteristics, type and content of asphalt, type of traffic, temperature and quality of construction. A thorough understanding of rutting phenomenon is required in order to improve pavement design and performance. The first apparent mode of rutting in the asphalt concrete layer is the densification (compaction) of the pavement resulting from the compressive load applied by the wheels. Although mixture densification (volume change) has some effect, rutting is principally caused by repetitive shear deformations under traffic loading. In the initial stage of trafficking, the increase of irreversible deformation [Sousa et al. 1991] below the tires is distinctly greater than the increase in the upheaval zones. In this mode, there is no significant lateral movement of material. Physically, this mode can be seen as reduction the voids in the asphalt concrete layer and reorientation of constituent particles as the wheels apply a compressive force. In this initial phase, therefore, traffic compaction has an important influence on rutting. After the initial stage, the volume decrement beneath the tires is approximately equal to the volume increment in the adjacent upheaval zones. This is an indication that compaction under traffic is completed for the most part and that further rutting is caused essentially by displacement with constancy of volume.

It is a shear type of deformation of the pavement which causes the material to move laterally from the wheels track and accumulate in between and to the side of the wheel paths. 
This phenomenon is clearly observed as the rising of the pavement material adjacent to the rut. In well-compacted pavements, shear deformation becomes the primary rutting mechanism since

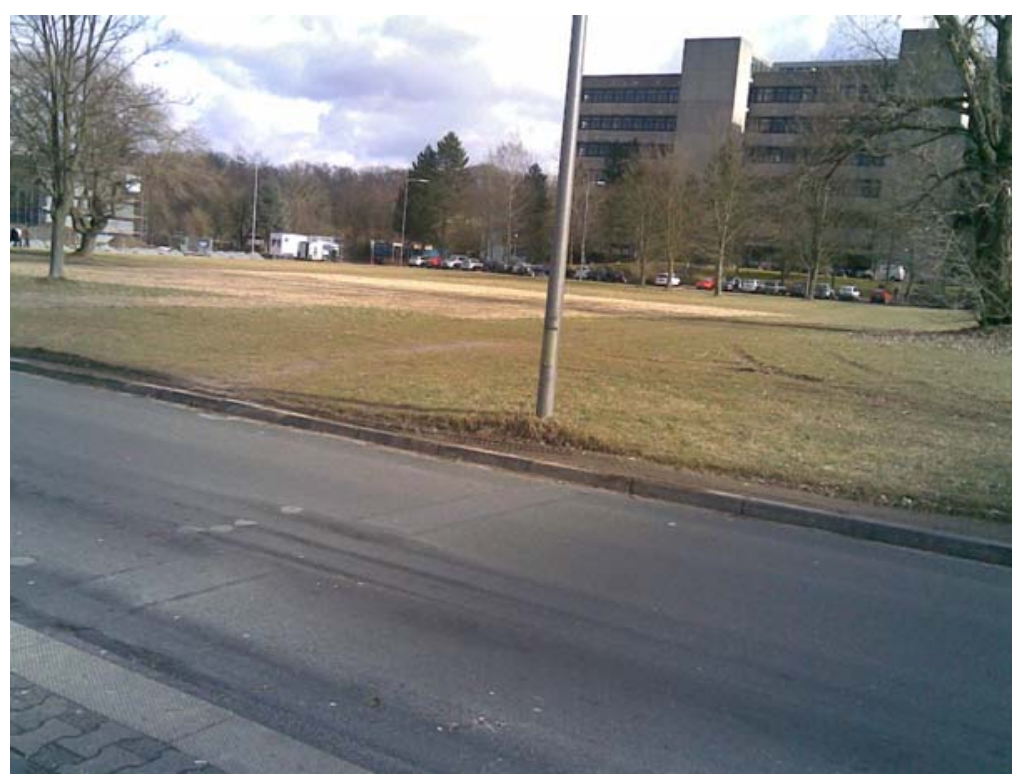

Figure 3.3 Permanent deformation at bus stop (TU-Lichtwiese, Feb. 2009)

the densification under the wheel path might not be more significant. This phase is considered to be representative of the deformation behavior for the greater part of the lifetime of a pavement.

Studies [Harvey et al. 2009, White et al. 2002] have shown that the shear strength of asphalt concrete pavements is a fundamental property in resisting rutting. Laboratory investigation indicated that improving the shear strength of the asphalt concrete mix can reduce surface rutting and some design agencies acknowledged the importance of the shear resistance of asphalt mixes as a fundamental property in resisting deformation of the pavement.
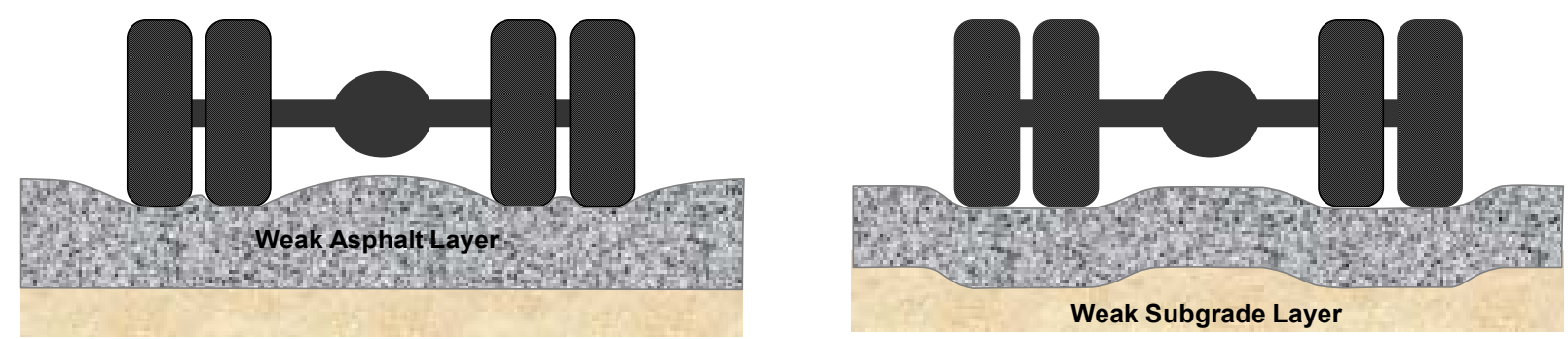

Figure 3.4 Modes of rut failure

Permanent deformation (rutting) of asphalt pavements has a major impact on pavement performance. It doesn't influence immediately the structural capacity of the pavement, but it can shorten significantly the useful service life of the pavement, besides causing an important loss in the riding quality of the road, by affecting vehicle handling characteristics, creates serious hazards for highway users. The deformation pattern creates functional problems such as loss of stability and durability, unevenness, worst riding comfort and drainage incapability resulting in moisture damage. It has an adverse effect on pavements by influencing the drainage properties 
leading to reduced frictional properties. Serious safety considerations also arise as a result of the accumulation of water in the ruts.

It has been [Freitas et al. 2005] described that a flexible pavement distress caused by a number of factors, including high contact stresses from truck tires, mix design characteristics (binder type and aggregate gradation), and poor construction quality (such as segregation and compaction methods). They concluded that rutted surface appears to contribute significantly the initiation of top down cracking.

It was found that the deformation [Sousa et al. 1991] through the asphalt-concrete layer was greatest near the loaded surface and gradually decreased at lower levels. Since rutting is caused by viscous/plastic flow, such a distribution of rutting with depth is reasonable: more resistance to viscous/plastic flow is encountered at greater depths and shear stresses are smaller at lower depths as well. It had been also reported by Sousa et al. that the deformation within an asphalt layer no longer increased with increasing layer thickness beyond a certain threshold. Measurements indicated that the surface rut depth reached a limiting value for certain asphaltconcrete thicknesses.

Rutting is a cumulative effect of several mechanisms. Among the factors influencing the amount of rutting are the magnitudes and pressures of tire loads, the volume of traffic, the thermal environment, and various mixture properties. Mixture properties, aggregate characteristics (specifically rough surface texture, angularity, and dense gradation) are particularly important contributors to permanent-deformation resistance. The asphalt materials tend to dilate under shear loading and at the same time the dilation is prevented (to some degree) by the surrounding material, leading to the development of normal stresses in the mix. The locking of the aggregates in place provides the primary stabilizing mechanism against rutting in asphalt concrete mixes. The amount and stiffness of the asphalt or modified asphalt binder are also important contributions against rut formation, with lower asphalt contents and stiffer binders providing improved resistance to permanent deformation.

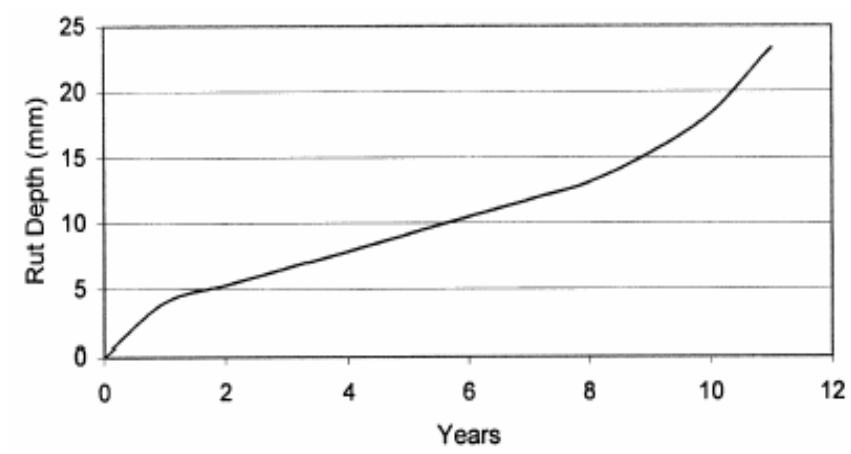

a. Classical rutting over time

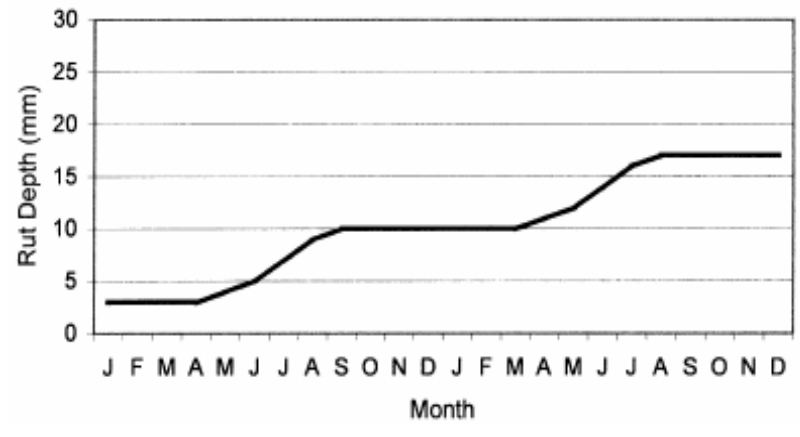

b. Potential seasonal effect on rutting

Figure 3.5 Rutting behavior with time

With increased tire pressures, axle loads, and load repetitions, there has been a resurgence of interest in the use of "large-stone" mixtures. It has been reported that some asphalt pavements constructed with soft asphalts, high volume concentrations of aggregate, low air-void contents, and large maximum aggregate size ( $1 \frac{1 / 2}{2}$ in. or larger) exhibited good rutting resistance. Based on observations, the use of larger maximum aggregate size (about two-thirds of layer thickness) would be beneficial in reducing the rutting propensity of mixtures subjected to high tire pressures.

When thicker asphaltic layers are constructed over stronger subbase and subgrade layers, the rutting has been seen confining within these layers. This phenomenon is observed in many European roads. For example in Germany, the substructure is constructed over a subgrade layer 
of minimum 45 Mpa modulus of elasticity for heavy traffic roads [RStO 1] while the superstructure is usually made from thicker asphalt layers and investigation [Werkmeister et al. 2003] particularly showed the formation of permanent deformation in the superstructures.

Several researchers have tried to improve rutting performance by using modifiers (polymers, micro fillers, etc.) intended to increase the viscosity of the asphalt binder at high temperatures without adverse effect at low temperatures. The binder content also affects the mixture's ability to resist permanent deformation. The Marshall or Hveem method is generally selected as a preliminary design tool in the determination of adequate asphalt content. It has been recommended that the mixtures have asphalt content such that the air-void content would be approximately 4 percent in order to preclude problems of instability and rutting. These criteria must necessarily be associated with mixtures of "adequate" stability resulting from the use of high quality aggregates. It has been indicated that larger asphalt contents producing lower air voids and increased rutting potential since the void space is becoming filled with asphalt as a result of increased asphalt content. The increase in asphalt content is equivalent to the introduction of lubricants between aggregate particles otherwise separated by a very tight network of air voids. This phenomenon causes the mixture with the higher asphalt content to be more susceptible to permanent deformation. Further more good resistance to permanent deformation requires low voids in the mineral aggregate (VMA). As indicated earlier, reducing air voids (up to 3\%) increases the resistance of the mixture to rutting.

\begin{tabular}{lll}
\hline & Conventional & HMAM \\
\hline Rut depth (mm) & 7.28 & 2.79 \\
Number of load application (cycles) & 20,000 & 20,000 \\
Load level (N) & 1372 & 1372 \\
Dynamic stability (N/mm) & 2747 & 7168 \\
\hline
\end{tabular}

Table 3.1 Results of wheel tacking test on hard grade asphalt concrete mix [Lee et al. 2007]

It has been found that relative displacements of mineral particles occurring when an asphalt mixture is handled at high temperature (during laying or compaction) or at moderate

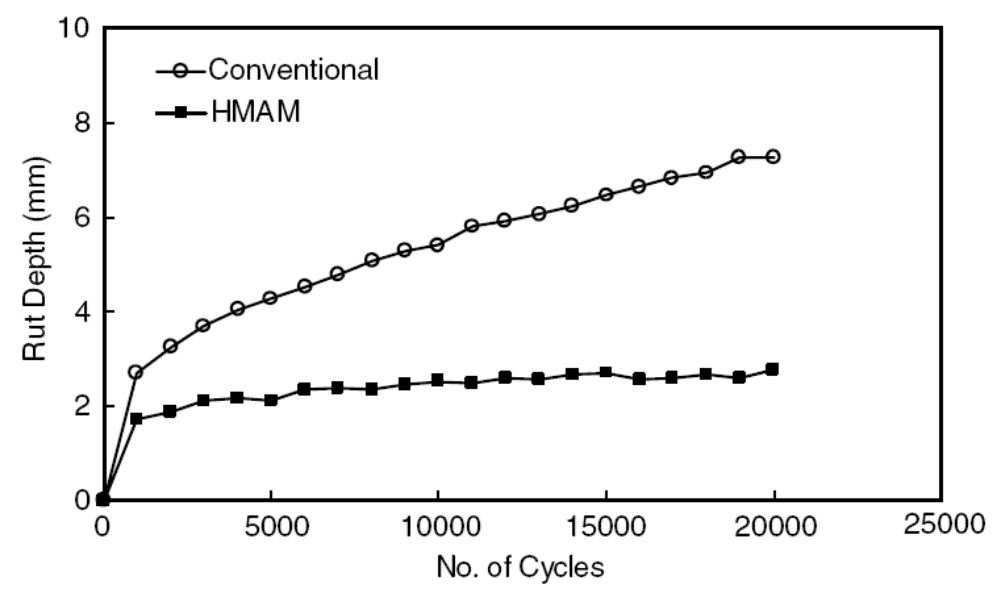

Figure 3.6 Cumulative rut depths with number of load applications for conventional mix and HMAM [Lee et al. 2007]

temperature but under prolonged loading. Therefore, to minimize rutting propensity, it is recommended the use of harsh mixtures (those of comparatively poor workability) and heavy 
rollers. Such a combination should result in an improved arrangement of the mineral skeleton and thereby an increase in internal friction. The well-designed, well produced mixture performs better (better durability and mechanical properties) when it is well-compacted.

Less viscous asphalts make the mixture less stiff and therefore more susceptible to irrecoverable deformations, therefore it is recommended harder (more viscous) asphalt cements in thicker pavements and hotter climates. It has been [Corté 2001] indicated that hard asphalts, produced in France for nearly 20 years, had offered for the French climatic context very interesting technical solutions for rutting mitigation of asphalt pavements and for construction of stiff asphaltic base layers.

\subsection{Fatigue Failure}

Pavement distress resulting from fatigue of asphalt concrete pavements has been a well recognized problem and the most costly form of distress to correct through rehabilitation. Fatigue failure occurs in areas subjected to repeated traffic loadings as well as due to climate fluctuation. It reveals as a series of interconnected or scattered cracks over/across surface layer. In order to address fatigue distress in mixture and pavement design procedures, it is necessary to describe the behavior of asphalt concrete mixtures under repeated stressing of the type encountered in situ. The failure criterion relates the allowable number of load repetitions to the

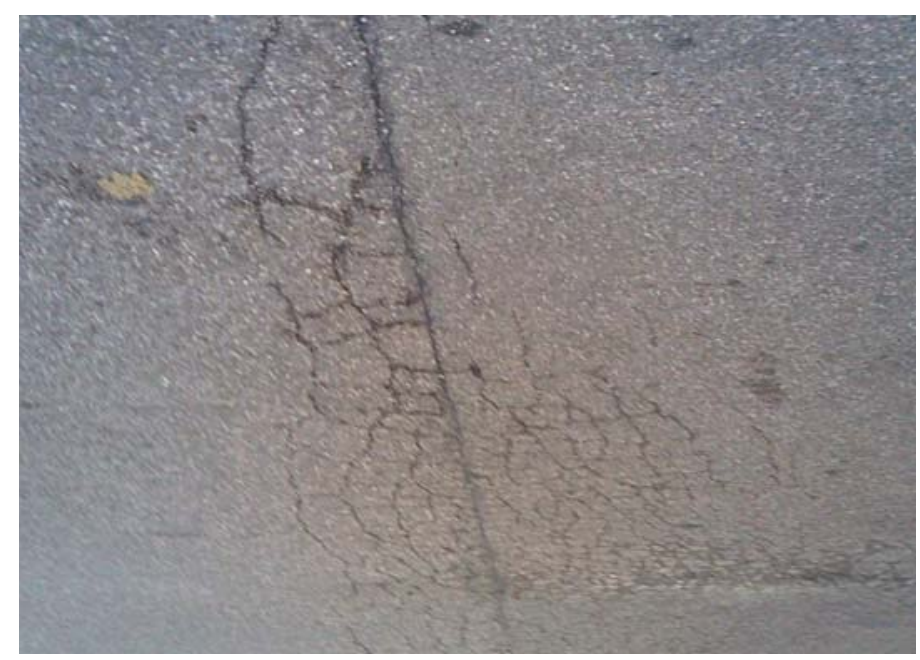

Figure 3.7 Fatigue cracking

tensile strain. Studies indicated that environmental conditions, tire-pavement interaction, mixture characteristics, aging of binder and construction practices are among the factors for the occurrence of the damage.

Cracks may start as microcracks that later coalesce to form macrocracks that propagate due to tensile and/or shear stress. As these cracks propagate, the pavement serviceability starts to reduce strongly. Until recently, load-associated fatigue cracking of hot-mix asphalt (HMA) concrete-surfaced pavements has been thought to occur when repeated wheel loads impose tensile strains of sufficient magnitude at the bottom of the asphalt concrete layer to initiate cracking that eventually propagates as one or more longitudinal parallel cracks up to the surface. However, Von Quintus [2001] indicated that load related HMA fatigue cracks can also be initiated at the surface of the pavement and propagate downward through the HMA layer. Such damages are caused by high tensile stresses in the top of the pavement structure. The penetration of water and other foreign debris into these cracks can further accelerate the propagation of the crack through the HMA surface layer.

During fatigue cracking, the first phase corresponds to degradation resulting from damage that is uniformly spread in the material. Hence, this phase is manifested by the initiation and 
propagation of a micro-crack network (in a diffuse way), which results in a decrease in modulus characteristics. In the next phase, from the coalescence of micro-cracks, a macro-crack appears which propagates within the material. The coalescence appears at a certain value of micro-cracking (or level of damage, or level of fatigue). The physical evolution law governing each of the two phases may be quite different and hence, the two phases are usually modeled within different theoretical frameworks. Damage and fracture mechanics respectively, can be used as frameworks for the deterioration phases. During experimental fatigue testing of bituminous mixes, the two phases can be considered as appearing successively, or for some test geometries, parallel in time.

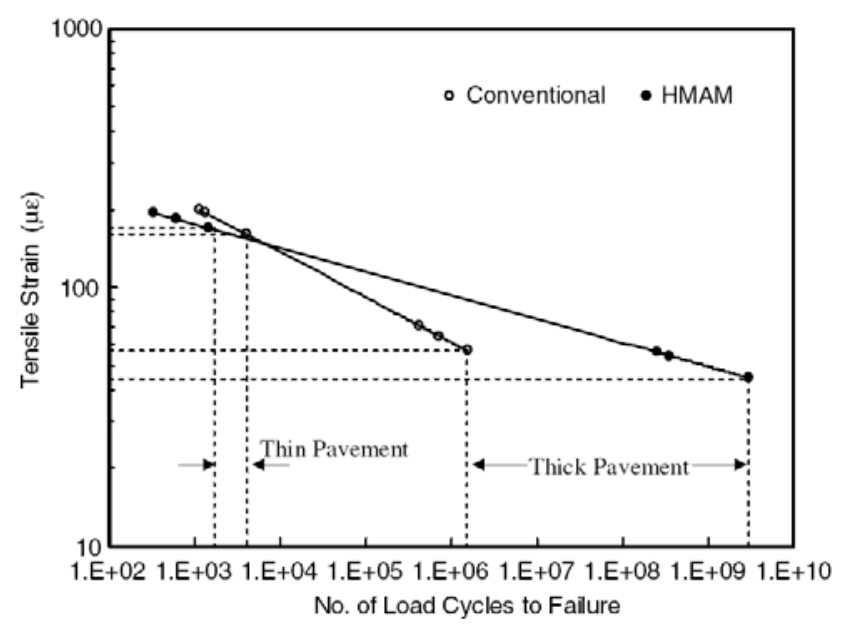

Figure 3.8 Results of fatigue test obtained from the full-scale performance test [Lee et al. 2007]

The fatigue deterioration is of great importance in pavement design and must be correctly predicted in order to ensure adequate structural stability. A precise understanding of the fatigue behavior of asphalt mixtures is required in order to improve asphalt mixture design and hot mix performance.

\subsection{Low Temperature Cracking}

Low temperature cracking is attributed to tensile stresses induced in the asphalt pavement

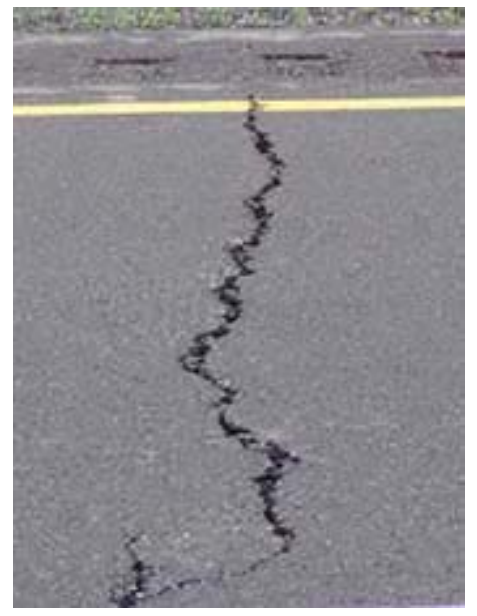

a. Transverse thermal cracking [50]

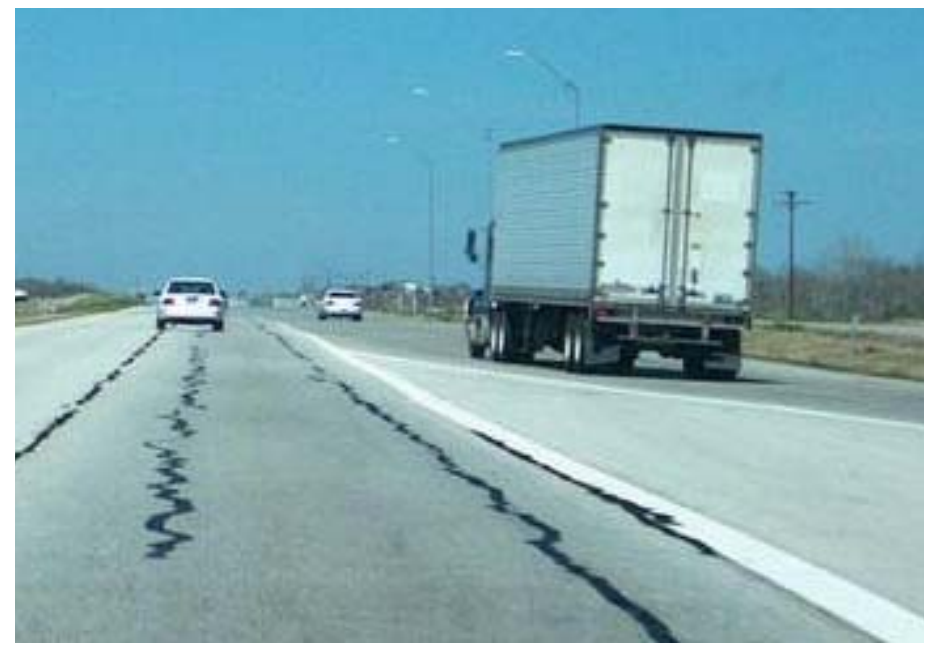

b. Top down cracking [49]

Figure 3.9 Asphalt concrete thermal associated cracking

that develop when the pavement is subjected to a cold temperature. As the pavement is cooled thermal stresses are induced as a result of the asphalt mixture's tendency to contract and 
deformation. The work done by the stress induces energy accumulation. If this energy accumulated in the pavement is equal to the critical energy of the asphalt mixture at that temperature, a low temperature cracking results.

Top down cracking is a common mode of HMA pavement distress in many asphalt concrete roads. Although not fully understood, there are three basic views on the of top-down cracking mechanism [49]:

- High surface horizontal tensile stresses due to truck tires (wide-based tires and high inflation pressures are cited as causing the highest tensile stresses).

- Age hardening of the asphalt binder resulting in high thermal stresses in the HMA (most likely a cause of the observed transverse cracks).

- A low stiffness upper layer caused by high surface temperatures.

\subsection{Asphalt Aging Failure}

Aging is one of the problems in asphalt pavement associated with the binder. It is either reversible or non-reversible. Irreversible aging is generally associated with oxidation at the molecular level. This oxidation increases an asphalt's viscosity with age up until a point when the asphalt is able to quench (or halt) oxidation through immobilization of the most chemically reactive elements. Reversible aging is generally associated with the effects of molecular organization. Over time, the molecules within asphalt will slowly reorient themselves into a better packed, more bound system. This results in a stiffer, more rigid material. This thixotropic aging can be reversed by heating and agitation.

Oxidative aging of asphalt is a primary cause of binder hardening in pavements [Chen and Huang 2000], thus contributing to various forms of pavement failures. An essential element of predicting long-term pavement performance is to understand binder oxidative aging and its effect on engineering properties. Material testing and characterization are always required to capture the resulting long term performance of asphalt concrete mixtures under aging hardening behaviors. This information can enable proper formulation of model used for asphalt pavement life time prediction and design and provide guidelines for material development.

\subsection{Miscellaneous Failures}

\section{Raveling}

Raveling or fretting is the progressive disintegration of the asphalt mixture from the pavement surface downward caused by the loss of the asphalt binder and/or dislodged aggregate particles. Small movements of individual aggregate particles, caused by traffic or water develop sufficient tensile stresses and strains which exceed the breaking strength of the asphalt binder. Slight to moderate raveling has a loss of the fine aggregate, while severe raveling includes the loss of the coarse aggregate. Raveling is also caused by moisture or solvent-induced stripping of the asphalt binder film from the aggregate, oxidation of the asphalt binder, and poor or low compaction during construction or insufficient asphalt binder content in the mixture. It usually occurs in cold or wet weather. Raveling caused by traffic is usually during cold weather, when the stiffness of the asphalt binder is high. It may also be caused by the abrasive action of tires. Moisture-induced damage or stripping is the most common cause of raveling. Pavements with high in-place air voids and low asphalt binder contents are likely candidates to experience moisture induced damage and raveling.

At low severity level, the fine aggregate or asphalt binder starts to wear away, exposing the tops of large aggregate. It is this condition that the surface starts to pit. The severity level become worse when the fine aggregate and asphalt binder has been considerably worn away and large losses of the coarse aggregate have occurred which ultimately lead to rough surface texture. The asphalt binder looses its binding effect and the aggregate becomes loose. 


\section{Flushing/Bleeding}

Flushing is the presence of excess asphalt binder on the pavement surface. It appears as a film of asphalt binder on the pavement surface, shiny and will become sticky when hot. Excessive asphalt binder content in the mixture or a very low air void content can cause flushing. Soft asphalt binders used in hot climates will also contribute to the problem. Flushing on roadways is a potential safety hazard, since it creates low skid resistance.

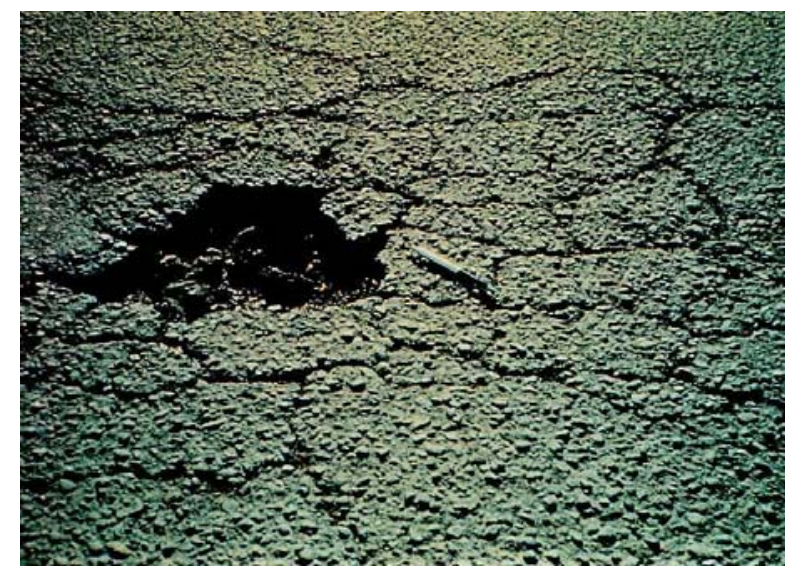

a. Pothole

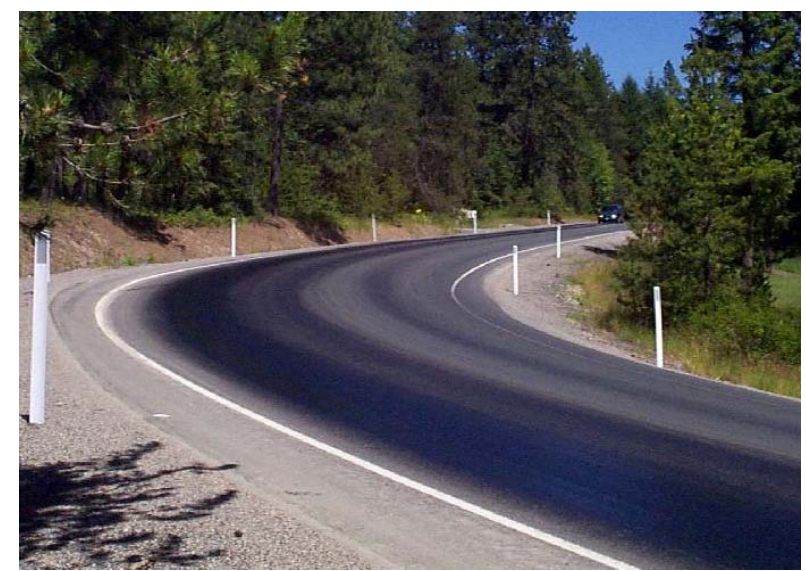

b. AC bleeding due to high asphalt content

Figure 3.10 Some forms of AC pavement failures [49]

\section{Polishing}

Polishing is caused by repeated wear on the pavement surface. It is a smooth slippery surface created by traffic polishing off the sharp edges of the coarse aggregate. Some aggregate has a greater tendency to polish than other types of aggregate. The tendency to polish is influenced by the geologic source of the aggregate. Limestone and chert have a greater tendency to polish than most other types of aggregates. Polished aggregate would have the asphalt binder film removed and be smooth to the touch. It will cause the pavement's skid resistance to be reduced. Polished aggregate, as a form of surface distress appears when the skid resistance values are low or have dropped significantly from previous ratings. It can be a serious surface distress for high-speed pavements. The elimination of the use of aggregates that easily polish from high-speed applications is the best preventive measure for polished aggregate distresses.

\section{Formation of Pothole}

Another form of pavement deformation is the formation of pothole which is caused when moisture enters a crack in the pavement, then freezes and expands in cold temperatures. The expansion puts pressure on the crack, causing the asphalt to break away, resulting in a pothole. Vehicle traffic over the pothole can cause the edges to crumble, which increases the size.

\section{Moisture Sensitivity of Asphalt Pavements}

The presence of water (or moisture) often results in premature failure of asphalt pavements in the form of isolated distress caused by debonding of the asphalt film from the aggregate surface or early rutting/fatigue cracking due to reduced mix strength. Moisture sensitivity has long been recognized as an important mix design consideration. Water can enter the pavement externally from the surface, laterally from poorly drained areas, underlying layers due to high ground water sources or can also be present internally in a newly constructed pavement as a 
result of inadequately dried aggregate. The presence of moisture, combined with the repeated action of traffic, accelerates damage to the asphalt pavement.

Moisture damage can come in many forms. Adhesive failure between the asphalt film and aggregate surface results in debonding which, in an advanced state, is identified as "stripping" which converts high strength asphalt treated pavement layer to a much weaker untreated aggregate section. When it occurs in isolated spots throughout the pavement, it can rapidly develop into potholes. Over more extensive areas, premature fatigue cracking or rutting may develop due to the reduced support strength of the overall pavement structure.

The most damaging and often hidden effect of moisture damage is associated with reduced pavement strength. The higher vertical compressive stress in the moisture damaged pavement can result in overstressing the underlying pavement layers and ultimately can create excessive permanent deformation or rutting in the wheel paths on the pavement surface.

Several theories have been proposed to describe the mechanisms of moisture damage. In reality, many of these mechanisms may occur during varying stages of moisture damage to the pavement structure. Some of the mechanisms suggested include detachment, displacement, emulsification etc.

Detachment of the asphalt film from the aggregate surface takes place when water rather than asphalt becomes the preferred coating of the aggregate. Displacement or debonding occurs when water actually displaces the asphalt that was originally bonded to the aggregate. The chemistry of the aggregate surface in the form of surface charges and the nature of the asphalt binder play major roles in the displacement process. Emulsification of the asphalt film can occur in a pavement due to the presence of emulsifying agents in the aggregate such as clay particles or agents in the asphalt binder itself. Traffic provides the action needed to promote emulsification. The resulting emulsion may migrate to the pavement surface and produce localized fat spots. The process of emulsification is normally reversible once the water is removed. 


\section{Modeling Approach}

Theoretical modeling has become a very important research tool and the fastest growing part of materials science. It analytically represents the realty of materials and is most often used in collaboration with laboratory experiment in order to predict materials response. The response of materials to external forces is simulated and the effect of load and the materials' response are evaluated.

Estimation and prediction of the maximum distresses likely to happen within the pavement layer during the pavement service life is most vital and a significant factor for proper design of the pavement. A realistic prediction of the long-term service life of asphalt pavements is one of the most challenging tasks for pavement engineers. It is performance models of asphalt concrete that provide the links among various processes involved in asphalt mixture design, pavement design, construction, and rehabilitation.

Several factors, such as rate of loading, temperature and moisture are considered in modeling techniques applied to asphalt paving mixtures and flexible pavements when investigating permanent deformation and fatigue cracking. Besides the commonly used phenomenological (S$\mathrm{N}$ ) approach recent works are based on elastic, viscoelasticity, elastoplastic, viscoplasticity and finite element modeling and simulation approach. Many researchers employed linear and nonlinear elastic and viscoelastic mechanical models to determine experimentally the constitutive parameters for asphalt concrete.

About $60 \%$ of the European countries use analytical based design models [111]. Empirical or unspecified models are used by $40 \%$ of European countries. The most favored analytical model for asphalt pavement has been the multi layer, linear elastic analysis. This model requires, among other input, stiffness modulus for bituminous materials, which depends on the temperature and frequency of loading.

\subsection{The Phenomenological (S-N) Approach}

The 'mechanistic' design method involves calculating pavement damage from critical strains using empirical equations called 'failure criteria' or 'performance relationships'. The most commonly used predictive equation [Archilla and Madanat 2000, Garba 2002] for the development of permanent deformation for the past several years was the power law described as:

$$
\varepsilon_{p}=C N^{B}
$$

Where $\varepsilon_{\mathrm{p}}$ - permanent strain

$\mathrm{N}$ - number of load repetition

C, B - material constants

In some literatures rutting is computed using the following equation [Archilla and Madanat 2000, Sousa et al. 1991]

$$
Y=a+b N^{0.5}
$$

Where $\mathrm{Y}$ - the rut depth

$\mathrm{N}$ - number of load applications

a, b - laboratory-determined parameters 
Most of the empirical based rutting models have been concerned on permanent deformation within the subgrade layer. Data from various reports, however, indicated that the subgrade contributed only parts of the total rutting, and the surface, base, and sub base layers contributed the major portion of the total rutting. In addition, these subgrade strain- based rutting methods did not account characteristics of paving materials during pavement service time.

On the other hand, the fatigue life of a pavement is empirically computed with the traditional power law. Fatigue models are divided into two main types, the strain-based models and the strain modulus based models. These models use a relation between the radial strain at the bottom of the asphalt concrete layer and the number of load applications to crack appearance in the pavement. This relation can be expressed in the following form [Ghuzlan and Carpenter 2003]:

$$
N_{f}=K_{1}\left(1 / \varepsilon_{o}\right)^{K_{2}}
$$

Where $\mathrm{N}_{\mathrm{f}}$ number of cycle to failure, $\varepsilon_{0}$-initial strain and $\mathrm{K}_{1}, \mathrm{~K}_{2}$ - are material constants found from laboratory investigation. Findings of the study show that there has been a high correlation between $K_{1}$ and $K_{2}$. The $K_{1}-K_{2}$ relation was found to be significantly affected by mode of loading, testing temperature and asphalt content in the mixture. The fatigue coefficients $K_{1}$ and $K_{2}$ vary from one model to another.

In some models the strain is replaced by the stress. It has been found that fatigue behavior is affected not only by strain but also by the modulus of the HMA and the following formula [Ghuzlan and Carpenter 2003] had been proposed:

$$
N_{f}=K\left(\varepsilon^{a}\left(E^{*}\right)^{b}\right)
$$

Where $\mathrm{E}^{*}$ is the dynamic stiffness modulus of the HMA, $\mathrm{K}$, $\mathrm{a}$ and $\mathrm{b}$ are material constants calibrated from laboratory investigation.

\subsection{Analytical Modeling Approach}

Analytical models play here an important role to foresee the distress-deformation behavior of the pavement. Constitutive modeling of the deformation behavior of asphalt concrete enables better understanding of the deterioration mechanisms taking place within the pavement structures and aid to design most economical and long lasting road. Many models have been developed so far for asphalt concrete to estimate the number of repetitions to failure in the rut/fatigue mode. A well constructed flexible pavement that is built above well defined threshold strength has a sustainable structural life provided that the distresses, in the form of fatigue, ruts and other modes of failures are kept at minimum value in such away that they don't affect structural integrity of the road.

\subsubsection{Continuum Mechanics}

Considering the weakness of the traditional approach, efforts have been undertaken to improve actual standards. There are some distinct theories that emerge from the host of possibilities for modeling material properties, namely those of elasticity, viscoelasticity, plasticity and viscoplasticity. Constitutive equations relate the strain to the stresses. Models involving elastic, viscous and plastic component has the potential to predict a realistic pavement response under a rolling wheel load.

Continuum mechanics assumes that the highly discontinuous structure of real materials can be replaced by a smoothed hypothetical continuum [56] and every portion of the continuum, however small, exhibits the macroscopic physical properties of the bulk material. In any branch of continuum mechanics, the field variables (such as density, displacement, velocity) are conceptual constructs. They are taken to be defined at all points of the imagined continuum and their values are calculated via axiomatic rules of procedure. 
The specification of the stress and strain states of a body is insufficient to describe its full behavior; we need in addition to link these two fields. This is achieved by introducing a constitutive relation, which prescribes the response of the continuum to arbitrary loading and thus defines the connection between the stress and strain tensor for the particular material. Classical continuum mechanics rests upon equations expressing the balance of mass, linear and angular momentums and energy of a body in motion.

Even though asphalt concrete is essentially a multi component, discrete material, in describing its response to external loads or temperature change the concept of a continuum mechanics approach has been overwhelmingly accepted. The stresses and strains, or their appropriate derivatives with respect to time, are then linked together in a constitutive equation which, supplemented with the equilibrium or motion equations and the boundary conditions, permits the evaluation of the pavement structure response either analytically or numerically. Among the various ways of relating the stresses and strains in the constitutive equation, an approach which first identifies three fundamental material properties or basic mechanical models has proven to be successful; i.e., elastic, viscous, and plastic [Chehab 2002, Drescher et al. 1993].

The strains due to elasticity are fully recoverable and independent of time. This means that in a loading/unloading cycle, no permanent strains are generated, and this is independent of the rate of loading and unloading [Drescher et al. 1993]. The strains due to viscosity are irrecoverable (permanent) and they depend on time. In other words, their magnitude depends on the load duration and the rate of loading and unloading. The strains due to plasticity are permanent but time independent. No matter what the rate of loading and unloading, the same magnitude of permanent strains is obtained for the same loading history. In real materials, two or all three properties may be expected, and the partition of the total strains into the various components is difficult, if not impossible, in some loading histories. This is because both the viscous and the plastic properties lead to permanent strains. Furthermore, materials show aging, i.e., change in properties with time, and temperature-dependent properties, both not being included in the simple models. The importance of identifying properties-related strains stems from the fact that the constitutive equations for each model are differently formulated. For example, neither elasticity nor viscosity, nor a combination of both, imposes any restrictions on the magnitude of stress. This, in turn, implies that the stresses and strains, or their time derivatives, are related through one continuous operator. On the other hand, plasticity implies a different operator for the loading and for unloading; these operators are separated by a function of stress-strain-yield condition.

\section{Elasticity Model}

An elastic material is distinguished [Haupt 1999] by the property that the current state of stress depends only on the current state of deformation, so that the memory part that goes back to the past deformation processes doesn't exist. The deformation of such materials is essentially of reversible. More over, an elastic structure is expected to give back the entire work done by forces and absorbed during a loading process, upon unloading. The most general constitutive equation in the theory of elasticity is derived from the strain energy and stress-strain relationship as follow:

$$
\sigma_{i j}=\frac{\partial W}{\partial \varepsilon_{i j}}
$$

Where $\mathrm{W}, \sigma_{\mathrm{ij}}$ and $\varepsilon_{\mathrm{ij}}$ are strain energy, stress and strain tensor, respectively. The stress strain relationship for linear homogenous elastic solid is derived from the above equation.

$$
\sigma_{i j}=E_{i j k l} \varepsilon_{k l}
$$


Where $\sigma, \mathrm{E}$ and $\varepsilon$ are stress, elastic modulus and strain, respectively

The simplest materials are ones in which the properties do not vary with direction, or linear elastic isotropic materials. An isotropic body has the same properties in every direction at any given point in the body. The normal stress causes extension in the direction of the applied stress and contraction in the perpendicular direction where as the shear stress causes only shearing deformation. Linear elastic isotropic materials are characterized by Young's modulus, Poisson's ratio, and the shear modulus. The following are relationships between the stress tensor and the strain.

$$
\left[\begin{array}{l}
\varepsilon_{11} \\
\varepsilon_{22} \\
\varepsilon_{33} \\
\varepsilon_{23} \\
\varepsilon_{13} \\
\varepsilon_{12}
\end{array}\right]=\left[\begin{array}{cccccc}
\frac{1}{\mathrm{E}} & \frac{-v}{\mathrm{E}} & \frac{-v}{\mathrm{E}} & 0 & 0 & 0 \\
\frac{-v}{\mathrm{E}} & \frac{1}{\mathrm{E}} & \frac{-\nu}{\mathrm{E}} & 0 & 0 & 0 \\
\frac{-v}{\mathrm{E}} & \frac{-v}{\mathrm{E}} & \frac{1}{\mathrm{E}} & 0 & 0 & 0 \\
0 & 0 & 0 & \frac{1}{\mathrm{G}} & 0 & 0 \\
0 & 0 & 0 & 0 & \frac{1}{\mathrm{G}} & 0 \\
0 & 0 & 0 & 0 & 0 & \frac{1}{\mathrm{G}}
\end{array}\right]\left[\begin{array}{c}
\sigma_{11} \\
\sigma_{22} \\
\sigma_{33} \\
\sigma_{23} \\
\sigma_{13} \\
\sigma_{12}
\end{array}\right]
$$

Where $\varepsilon_{\mathrm{ij}}, \sigma_{\mathrm{ij}}$ - are strains and stresses, respectively,

E, G - Elastic and shear modulus of materials, respectively and

ív - Poisson's ratio of the materials.

The three material constants are related as:

$$
\mathrm{G}=\frac{\mathrm{E}}{2(1+v)}
$$

\section{The Layer-Strain Method}

It consists of predicting rut depths using permanent-deformation characteristics determined from laboratory tests together with an analysis procedure for the pavement structure using either linear or nonlinear elastic theory [Sousa et al. 1991]. To predict the amount of permanent deformation that would occur after a given number of wheel load applications, each layer of the pavement structure is divided into several sublayers, and the stress state is calculated at the center of each sublayer directly beneath the wheel load using elastic analysis. With the average stress state at the center of each sublayer, the corresponding axial plastic strain can be ascertained from the results of laboratory tests. The total rut depth for a given number of load repetitions is obtained by summing the products of the average permanent strain occurring at the center of each sublayer and the corresponding sublayer thickness using equation 4.9.

$$
\Delta \mathrm{P}=\sum_{\mathrm{i}=1}^{\mathrm{n}}\left[\left(\varepsilon_{\mathrm{i}}^{\mathrm{p}}\right)\left(\Delta \mathrm{Z}_{\mathrm{i}}\right)\right]
$$

Where $\Delta \mathrm{P}$ - total rut depth,

$\varepsilon_{i}^{\mathrm{p}}$ - average permanent (plastic) strain in the $\mathrm{i}^{\text {th }}$ sublayer,

$\Delta \mathrm{Z}_{\mathrm{i}}$ - thickness of the $\mathrm{i}^{\text {th }}$ sublayer, and

$\mathrm{n}$ - total number of sublayers.

This approach has been adopted in various forms by many researchers and is considered a simplified engineering approach for predicting rut depth which permits the flexibility of using either linear or nonlinear elastic analysis.

Asphalt is a visco-elasto or viscoelastoplastic material with behavior depending on temperature and rate of loading. However, in numerous pavement analysis programs, hot-mix asphalt is 
assumed to be linearly elastic. This assumption is considered to be oversimplified for more detailed analysis. Most of the design procedures used in road engineering are also based on linear elastic multi-layer analysis and simple laboratory tests. Unfortunately, design life and material degradation, which are essential issues in estimating the lifetime of a pavement, cannot be addressed by linear elasticity. In the damage accumulation process the complex nature of asphalt concrete is essential and accurate performance predictions are possible only when that nature is modeled. This means that, besides the temperature and strain rate dependency, the state of stress dependency of the material response must be also taken into account. So far, this is often neglected although it is well known that the strength of asphalt concrete increases with increasing confinement. When it comes to failure predictions, this aspect of the material behavior can no longer be neglected. Therefore recent researches have [Abas et al. 2004, Elseif et al. 2006, Chehab 2002, Chehab and Kim 2005, Kim and Little 2004, Lee and Kim 2002] been focusing on simulating the behavior of asphalt concrete materials using viscoelastic, viscoplastic or visco-elasto-plastic models.

\section{Viscoelasticity Model}

The theory of viscoelasticity can be used to deal with the reversible evolutions of viscoelastic solids as functions of time [Lermaitre and Chaboche 1990]. It is a rate-dependant material behavior without equilibrium hysteresis.

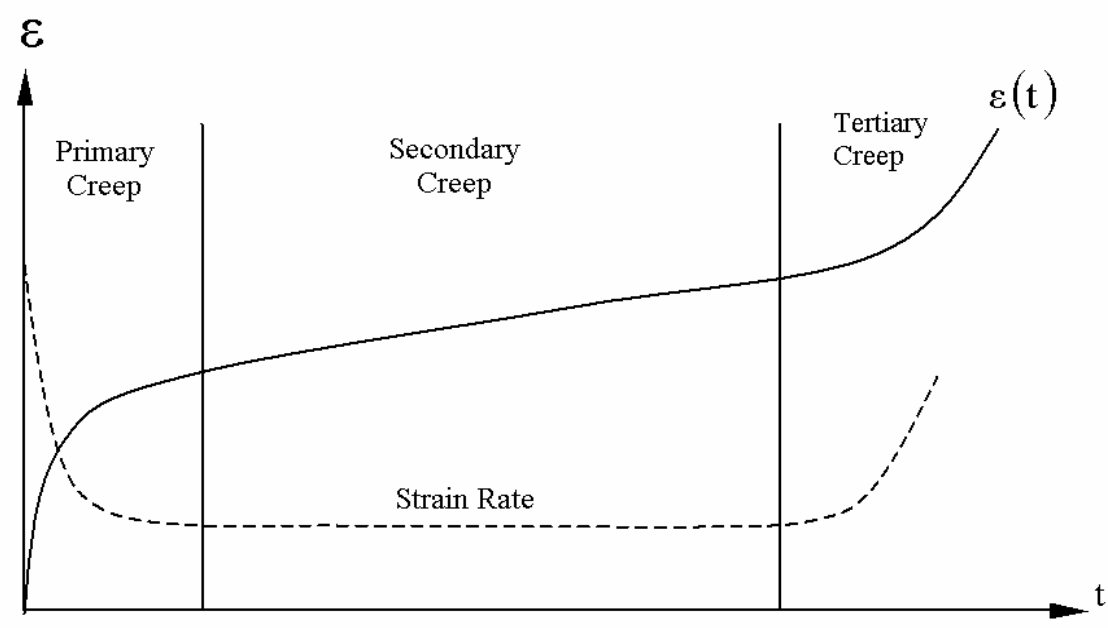

Figure 4.1 Creep curve

A systematic development of special material models of viscoelasticity can be based on the general constitutive equations for simple materials, in which the total stress response is split into an elastic part and a memory part, respectively, as shown in Fig. 4.2 [Vormwald 2006].

$$
\sigma_{\mathrm{E}}=\mathrm{E} \varepsilon \quad \text { and } \quad \sigma_{\mathrm{D}}=\eta \frac{\mathrm{d} \varepsilon}{\mathrm{dt}}
$$

Where the notations $\mathrm{E}$ and $\eta$ are elastic and viscous constants, respectively

Linear viscoelastic deformation is represented by the constitutive equations combining spring and dashpot elements. The Maxwell model can be represented by a purely viscous damper and a purely elastic spring connected consecutively, as shown in the diagram. In a Maxwell material, stress $\sigma$, strain $\varepsilon$ and their rates of change with respect to time $t$ are governed by equations of the form. 


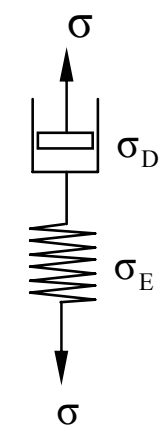

a. Maxwell model

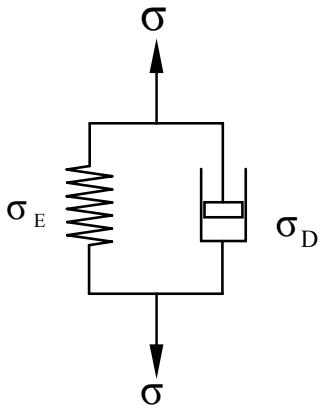

b. Kelvin-Voigt model

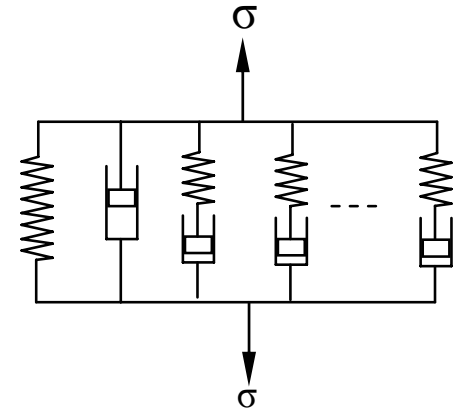

c. General Kelvin-Voigt model

Figure 4.2 Simplified material models

$$
\begin{aligned}
& \varepsilon=\varepsilon_{\mathrm{D}}+\varepsilon_{\mathrm{E}} \Rightarrow \frac{\mathrm{d} \varepsilon}{\mathrm{dt}}=\frac{\mathrm{d} \varepsilon_{\mathrm{D}}}{\mathrm{dt}}+\frac{\mathrm{d} \varepsilon_{\mathrm{E}}}{\mathrm{dt}} \text {, and } \sigma=\sigma_{\mathrm{D}}=\sigma_{\mathrm{E}} \\
& \sigma+\frac{\eta}{\mathrm{E}} \frac{\mathrm{d} \sigma}{\mathrm{dt}}=\eta \frac{\mathrm{d} \varepsilon}{\mathrm{dt}} \Rightarrow \sigma(\mathrm{t})=\mathrm{e}^{-\frac{\mathrm{E}}{\eta}}\left[\int\left(\mathrm{Ee}^{\frac{\mathrm{E}^{\mathrm{t}}}{\eta}} \frac{\mathrm{d} \varepsilon}{\mathrm{dt}}\right) \mathrm{dt}+\mathrm{C}\right]
\end{aligned}
$$

The constitutive equation of the parallel model of spring and dashpot elements (Kelvin-Voigt model) stress $\sigma$, strain $\varepsilon$ and their rates of change with respect to time $t$ are governed by equations of the form:

$$
\begin{aligned}
& \sigma=\sigma_{\mathrm{D}}+\sigma_{\mathrm{E}} \text {, and } \varepsilon=\varepsilon_{\mathrm{D}}=\varepsilon_{\mathrm{E}} \\
& \sigma=\mathrm{E} \varepsilon+\eta \frac{\mathrm{d} \varepsilon}{\mathrm{dt}} \Rightarrow \varepsilon(\mathrm{t})=\mathrm{e}^{-\frac{\mathrm{E}}{\eta}}\left[\int\left(\frac{\sigma}{\eta} \mathrm{e}^{\frac{\mathrm{E}}{\eta}}\right) \mathrm{dt}+\mathrm{C}\right]
\end{aligned}
$$

The above conceptual model serves to introduce the second or higher order derivatives of stress and strain into the constitutive equation. In general, the linear viscoelastic material has a linear stress-strain relation of the form,

$$
\sum_{j=0}^{n} P_{j} \frac{d^{j} \sigma}{(d t)^{j}}=\sum_{k=0}^{n} P_{k} \frac{d^{k} \varepsilon}{(d t)^{k}}
$$

In fact, different recoverable and creep strains, both instantaneous and time dependent, can be described by a generalized viscoelastic superposition principle [Drescher et al. 1993], whose mathematical form is :

$$
\varepsilon(t, \sigma)=\int_{-\infty}^{t} J_{1}\left(t-\tau_{1}\right) \frac{d \sigma\left(\tau_{1}\right)}{d \tau_{1}} d \tau_{1}+\int_{-\infty}^{t} \int_{-\infty}^{t} J_{2}\left(t-\tau_{1}, t-\tau_{2}\right) \frac{d \sigma\left(\tau_{1}\right)}{d \tau_{1}} \frac{d \sigma\left(\tau_{2}\right)}{d \tau_{2}} d \tau_{1} d \tau_{2}+\ldots
$$

The first term represents a linear contribution of stress increments. The remaining terms represent the combined effect of the subsequent stress increments during loading and unloading. Taking the appropriate number of terms in above equation, any departure from the linear response can be accurately described. All non-linear viscoelastic descriptions predict the difference between the recoverable and creep strains to diminish with the decrease of time to unloading. 

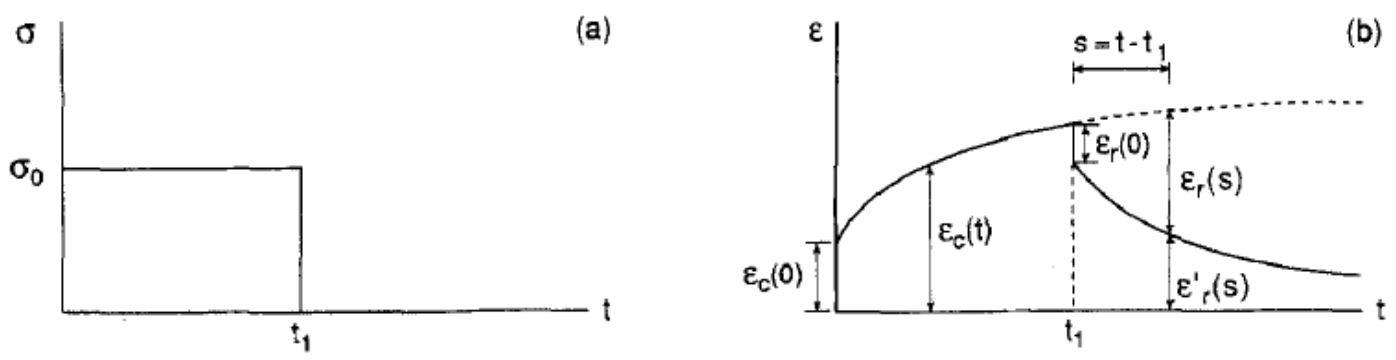

Figure 4.3 Single-step creep test: (a) Loading/unloading history; (b) Strain-time response [Drescher et al. 1993]

It is also known that when a material is in linear viscoelastic range, the effects of time or frequency and temperature can be expressed through one joint parameter. As such, the same material property values can be obtained either at low temperatures and long times or at high testing temperatures but short times.

Finite element programs used for simulations usually require as input the temperature dependent shear relaxation modulus, $\mathrm{G}(\mathrm{T}, \mathrm{t})$ and the bulk relaxation modulus, $\mathrm{K}(\mathrm{T}, \mathrm{t})$. In ABAQUS [2], the basic hereditary integral formulation for linear isotropic viscoelasticity is given as:

$$
\sigma(\mathrm{t})=\int_{0}^{\mathrm{t}} 2 \mathrm{G}\left(\tau-\tau^{\prime}\right) \dot{\mathrm{e}} \mathrm{dt} \mathrm{t}^{\prime}+\mathrm{I} \int_{0}^{\mathrm{t}} \mathrm{K}\left(\tau-\tau^{\prime}\right) \phi^{\prime} \mathrm{dt}^{\prime}
$$

Where e and $\phi$ are the mechanical deviatoric and volumetric strains; $K$ and $G$ are the bulk and shear modulus, respectively; $\tau$ the reduced time; and 'denotes differentiation with respect to t'.

The reduced time is related to the actual time through the integral differential equation.

$$
\tau=\int_{0}^{\mathrm{t}} \frac{\mathrm{dt}^{\prime}}{\mathrm{A}_{\theta}\left(\theta\left(\mathrm{t}^{\prime}\right)\right)}, \quad \frac{\mathrm{d} \tau}{\mathrm{dt}}=\frac{1}{\mathrm{~A}_{\theta}(\theta(\mathrm{t}))}
$$

The relaxation functions $K(t)$ and $G(t)$ can be defined individually in terms of a series of exponentials known as the Prony series:

$$
K(t)=K_{\infty}+\sum_{i=1}^{n_{K}} K_{i} e^{-t / \tau_{i}^{K}} \quad G(t)=G_{\infty}+\sum_{i=1}^{n_{G}} G_{i} e^{-t / \tau_{i}^{G}}
$$

Where $K$ and $G$ represent the long-term bulk and shear moduli.

Temperature [Nazarian and Alvarado 2006] is one of the most dominant factors that impact the variation in modulus of asphalt concrete (AC) layers. An accurate relationship between temperature and modulus is necessary to successfully characterize asphalt concrete pavements. The variation in modulus is typically related to the average temperature of a layer. However, the temperature gradient within a layer also plays a role in that relationship.

Typical 'retarded elastic' response is illustrated below, by plotting creep compliance as a function of time for various temperatures 


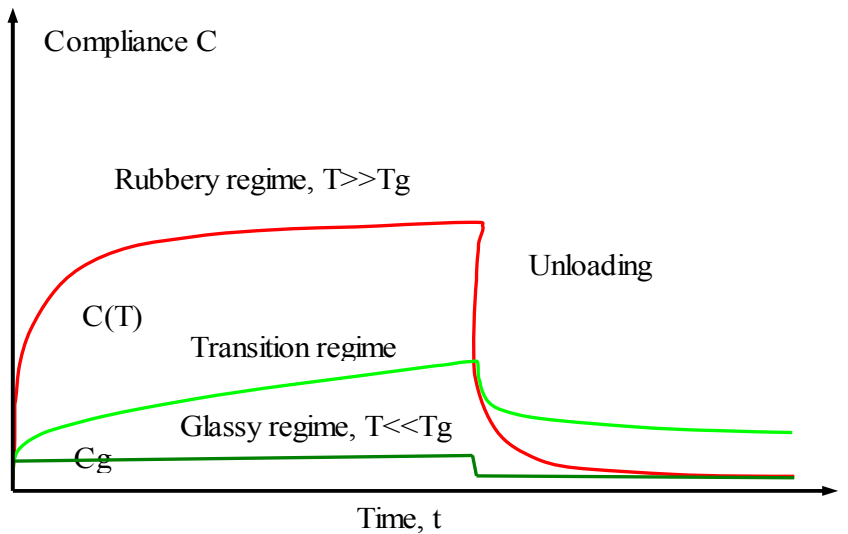

Figure 4.4 Effect temperature on viscoelastic response of a materials

One can plot the modulus as a function of temperature or inverse frequency, with almost identical results - the typical trend is illustrated below.

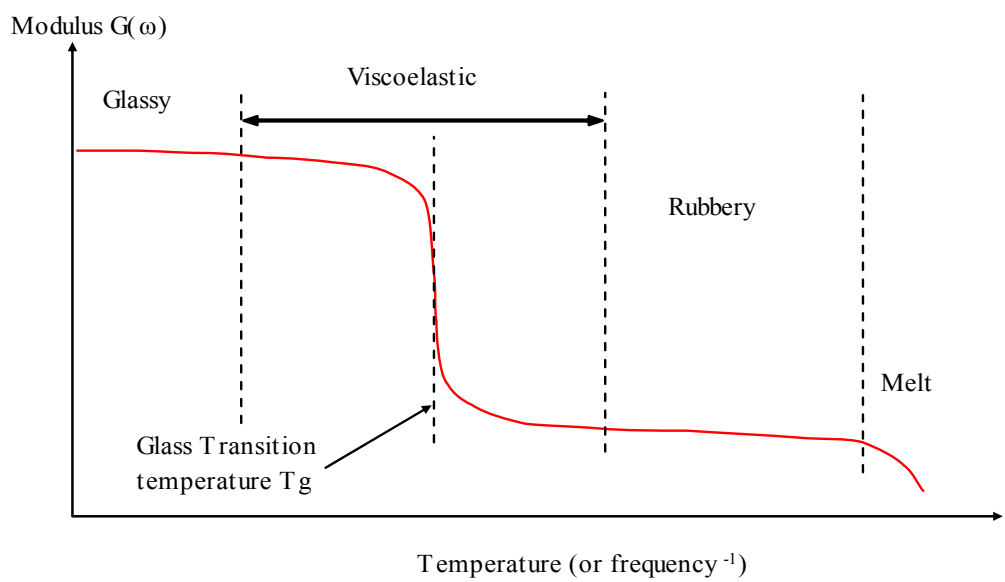

Figure 4.5 Glass transition with respect to temperature

The relationship between temperature and loading-response can be demonstrated by measuring the time dependent relaxation modulus for the material at different temperatures which later can be collapsed onto a single master-curve. The viscoelastic material property (e.g., relaxation modulus and creep compliance) as a function of time (or frequency), at various temperatures can be shifted along the horizontal time axis (log scale) to form a single characteristic master curve as a function of reduced time at a desired reference temperature. Theoretically, the time-temperature shift factors, which are a function of the material itself, are evaluated as follows.

For the relaxation modulus at a certain time and temperature,

$$
E(t, T)=E(\xi)
$$

Where $\xi=\frac{t}{a_{T}}$

T-time before shifting for a given temperature, $\mathrm{T}$

$\xi$ - reduced time at reference temperature $\mathrm{T}_{0}$ and

$\mathrm{a}_{\mathrm{T}}$ - shift factor for temperature $\mathrm{T}$ 
The well-known WLF equation developed by William, Landal and Ferry estimates the shift factor as,

$$
\log a_{T}=\log \frac{t_{T}}{t_{T_{0}}}=\frac{C_{1}\left(T-T_{0}\right)}{C_{2}+\left(T-T_{0}\right)}
$$

Where $\mathrm{C}_{1}$ and $\mathrm{C}_{2}$ are constants dependant on the reference temperature $\mathrm{T}_{0}$ expressed in degree Kelvin. The WLF equation can only be applied to temperatures above the glass transition temperature, which is around $-30{ }^{\circ} \mathrm{C}$ for asphalt.

The shift factors can also be determined experimentally through graphical shifting of storage modulus curves and later refined through error minimization using fitting techniques. The following is another way finding the shift factor [Chehab 2002].

$$
\log \left(f_{R}\right)=\log \left(f \cdot a_{T}\right)
$$

Where $f_{R}$ - reduced frequency at the reference temperature

$\mathrm{f}$ - Frequency at a given temperature $\mathrm{T}$ before shifting

$a_{T}-$ shift factor for temperature $T$

$$
E^{\prime}=a_{1}+\frac{a_{2}}{\left\{a_{3}+\frac{a_{4}}{\exp \left[a_{5}+a_{6} \log _{10}^{\left(f_{R}\right)}\right]}\right\}}
$$

Where $f_{R}$ - the reduced frequency

$\mathrm{a}_{1}$ through $\mathrm{a}_{6}-$ regression coefficients

$\mathrm{E}^{\prime}$ - the storage modulus

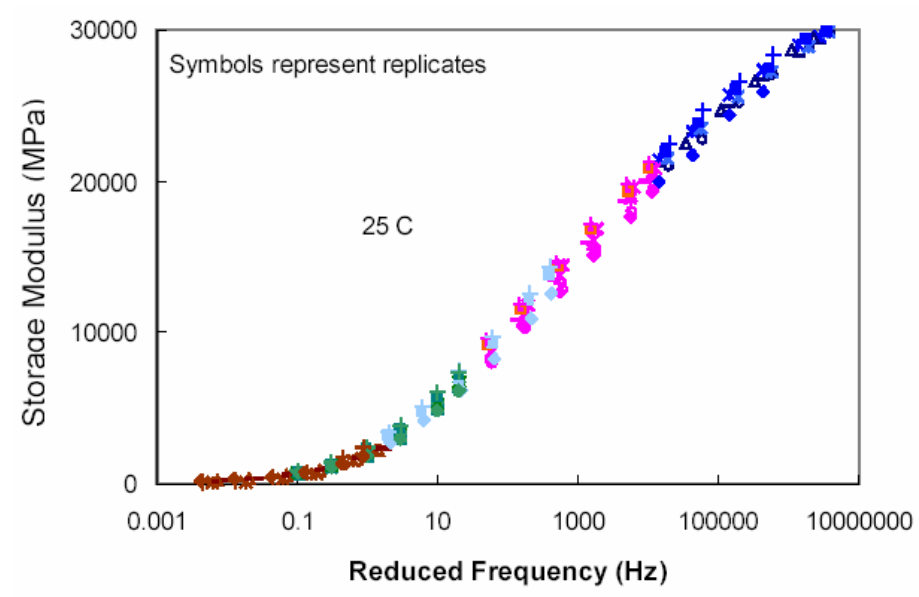

Figure 4.6 Storage modulus as a function of reduced frequency [Chehab 2002] 


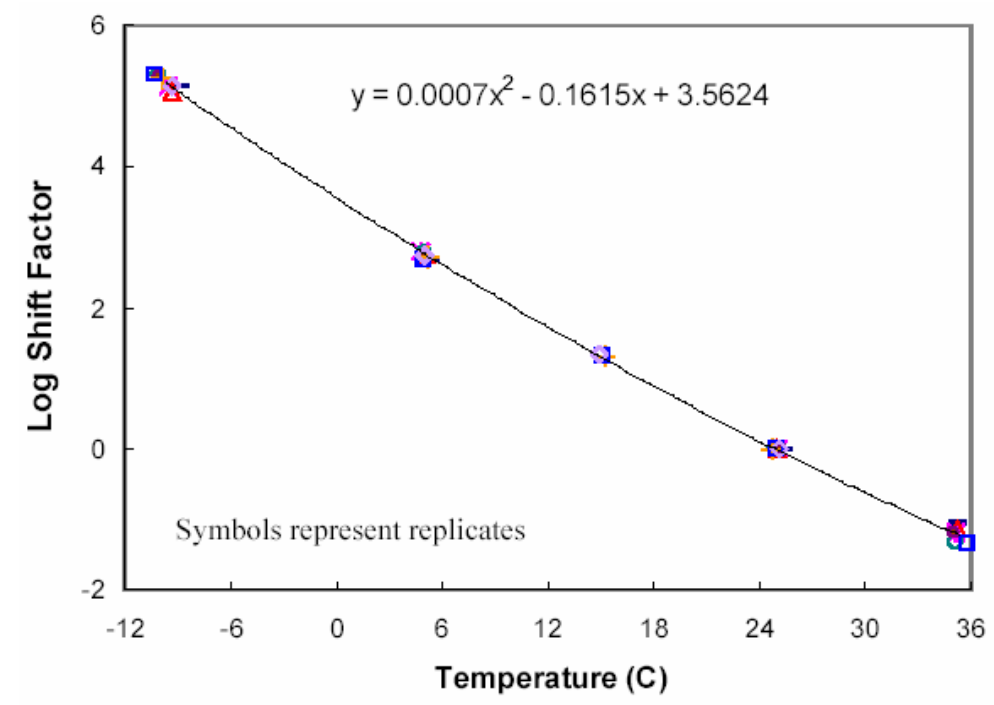

Figure 4.7 Log shift factor as a function of temperature obtained by constructing the storage modulus master curve at $25^{\circ} \mathrm{C}\left(-10,5,15,25,35^{\circ} \mathrm{C}\right)[$ Chehab 2002].

\section{Burgers Model}

The rheological parameters were obtained by approximating the creep curve with theoretical curves determined for the viscoelastic Burgers model (Figure 4.8a). Equations 4.25 through 4.28 describe the relation between time and the Burgers model's rheological parameters [Szydlo and Mackiewicz 2005, Liu and You 2008]:

- For load $\mathrm{t}<\mathrm{t}_{0}$

$\varepsilon(t)=\sigma_{0}\left[\frac{1}{E_{1}}+\frac{t}{\eta_{1}}+\frac{1}{E_{2}}\left(1-e^{-t E_{2} / \eta_{2}}\right)\right]$

- For load $\mathrm{t}>\mathrm{t}_{0}$

$\varepsilon(t)=\sigma_{0}\left[\frac{t_{0}}{\eta_{1}}-\frac{1}{E_{2}} e^{-t E_{2} / \eta_{2}}\left(1-e^{-t_{0} E_{2} / \eta_{2}}\right)\right]$

Where $t_{0}$ and $t$ are the maximum loading period and any time during loading or unloading period

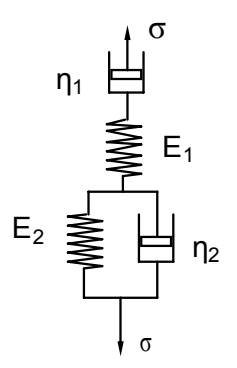

a. Burger model

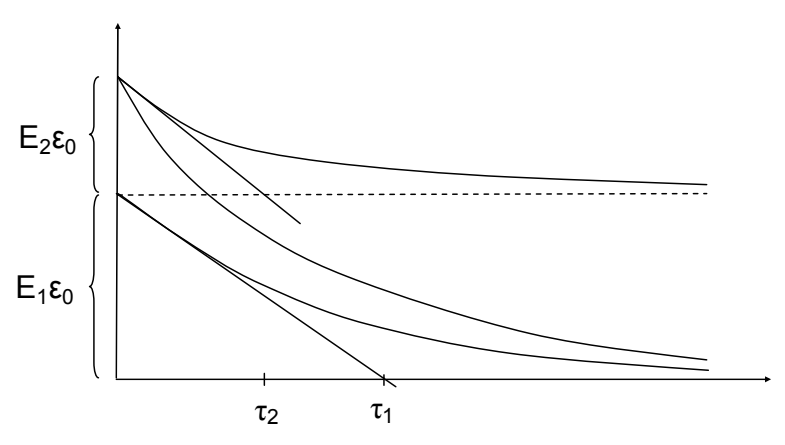

b. Relationship of Burgers parameters

Figure 4.8 Burger model and materials parameter 
In case of relaxation test where a constant strain is applied on a Burger model which consists of two Maxwell models arranged in parallel, the time dependent modulus of elasticity (as shown in Figure 4.8b) is expressed as [Vormwald 2006]:

$$
E(t)=E_{1} e^{\left(\frac{-t}{\tau_{1}}\right)}+E_{2} e^{\left(\frac{-t}{\tau_{2}}\right)}
$$

Or,

$$
E(t)=E_{1} e^{\left(\frac{-E_{1} t}{\eta_{1}}\right)}+E_{2} e^{\left(\frac{-E_{2} t}{\eta_{2}}\right)}
$$

Where $E(t)$-time dependent elasticity modulus

$\eta_{1}, \eta_{2}$ - Burgers viscoelastic parameters

$\mathrm{E}_{1}, \mathrm{E}_{2}$ - elastic modulus parameters in Burgers model

t-Time

\section{Plasticity Model}

Plastic materials are those materials which reveal instantaneously stable permanent deformations after the application of a load and in equilibrium with the load. The theory of plasticity [Haupt 1999, Lermaitre and Chaboche 1990] model is rate-independent material behavior which undergoes irreversible shape changes in response to applied force. Plasticity expands on the theory of elasticity by taking internal dissipation into consideration. However, internal friction is represented from a completely different viewpoint, compared with viscoelasticity: where as viscoelastic material behavior is characterized by fading memory properties, the theory of plasticity tends to express a perfect memory of material body. Plastic behavior of a material is characterized by a non-unique stress-strain relation with the presence of irreversible strains on load removal. This is obtained by the usual decomposition of the strain vector $\varepsilon$ in an elastic, reversible part, $\varepsilon^{\mathrm{e}}$ and a plastic, irreversible part, $\varepsilon^{\mathrm{p}}$.

$$
\varepsilon=\varepsilon^{e}+\varepsilon^{p}
$$

Where the elastic strain rate is related to the stress rate by the elastic (constitutive) stiffness matrix D as,

$$
\sigma=D \varepsilon^{e}
$$
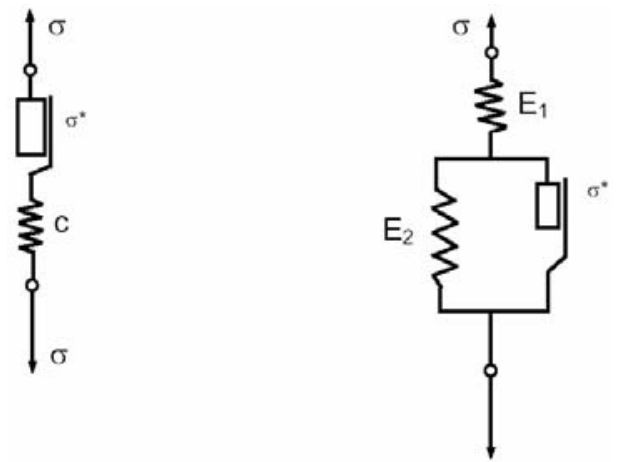

Figure 4.9 Elastic-plastic Model [Vormvald 2006]

The onset of plastic flow is governed by a yield criterion which defines a complete state of stress at which a point in a solid body is either elastic below, or plastic above, a limit stress. A yield criterion, often expressed as yield surface, is a hypothesis concerning the limit of elasticity under 
any combination of stresses at which a material begins to deform plastically. The stress state corresponding to an elastic behavior of the material characterizes the elastic domain which in turn defines the yield criterion in the stress space. If the yield function is isotropic, then the yield function depends on the stress only through the principal stresses $\sigma_{1}, \sigma_{2}, \sigma_{3}$ or equivalently its variance. One of the most widely used yield criteria is the von Misses which expresses as [Valoroso 2006]:

$$
\phi(\sigma)=\sqrt{\frac{\left(\sigma_{1}-\sigma_{2}\right)^{2}+\left(\sigma_{2}-\sigma_{3}\right)^{2}+\left(\sigma_{1}-\sigma_{3}\right)^{2}}{3}}-\sqrt{\frac{2}{3}} Y_{0}=0
$$

Or, the yield is also defined as:

$$
\phi(\sigma)=\sqrt{2 J_{2}}-\sqrt{\frac{2}{3}} Y_{0}=\|S\|-\sqrt{\frac{2}{3}} Y_{0}=0
$$

Where $\phi(\sigma)$-Yield function

S-represent the stress deviator

$\mathrm{Y}_{0}$ - the uniaxial yield limit

$\mathrm{I}_{1}, \mathrm{~J}_{2}, \mathrm{~J}_{3}$ - invariants defined as follows

$$
\begin{aligned}
& I_{1}=\operatorname{tr}(\sigma)=\sigma_{11}+\sigma_{22}+\sigma_{33} \\
& J_{2}=\frac{1}{2} S: S=\frac{1}{2} S_{i j} S_{i j} J_{3}=\frac{1}{3} \operatorname{tr}\left(S^{3}\right)=\frac{1}{3}(S \cdot S): S=\frac{1}{3} S_{i j} S_{j k} S_{k i} \\
& S_{i j}=\sigma_{i j}-\frac{1}{3} \operatorname{tr}(\sigma)=\sigma_{i j}-\frac{1}{3} I_{1}
\end{aligned}
$$

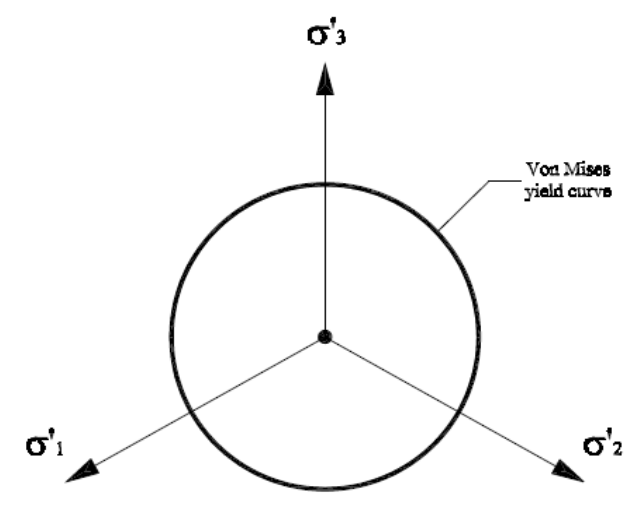

Figure 4.10 The von Mises yield function in the deviatoric space

\section{Visco-Elasto-Plasticity Model}

The pavement response under traffic loading consists of both recovery and irrecovery components. If the recoverable and creep strains do not satisfy the above equation, the only conclusion that can be drawn is that the material is not linearly viscoelastic. It is possible, for instance, to regard it as nonlinearly viscoelastic or as plastic-viscoelastic. An important 
advantage of this approach is that moving wheel loads can be considered directly. This results in the correct time-rate of loading to be applied to each material element and permits estimates to be made of the lateral plastic flow of material from beneath the moving wheel. Nonlinear viscoelastic response characteristics may provide a more realistic estimate of pavement response. Moving wheel loads can be considered in conjunction with time-dependent material properties to define the states of stress and strain at particular points in the pavement structure. Material properties can be defined either in terms of model consisting of finite numbers of Maxwell and/or Kelvin elements in various arrangements or in terms of generalized compliance relationships.

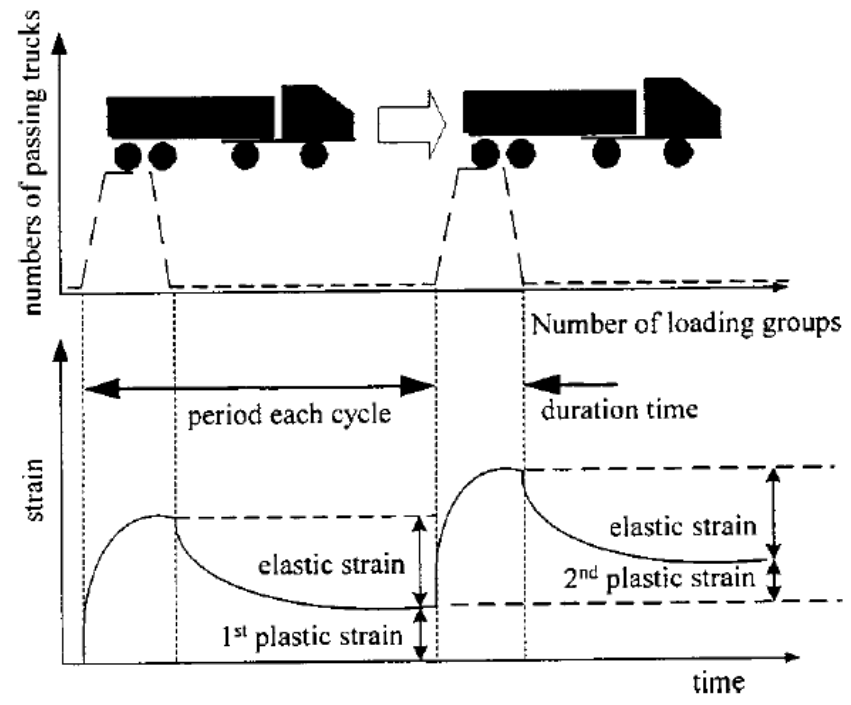

Figure 4.11 Accumulated plastic strain on pavement surface under continuous traffic loading [Chen et al. 2004]

The theory of viscoplasticity [Haupt 1999] depicts rate-dependent material behavior with equilibrium hysteresis. It exhibits [Lermaitre and Chaboche 1990] permanent deformations after the application of loads (like plastic solids) but it continues to undergo a creep flows as a function of time under the influence of the applied load. The framework of viscoplastic constitutive model is constructed based on the overstress theory on the premise that the sharp yield point and the subsequent abrupt yield drop result from rapid dislocation, multiplication and the stress dependence of dislocation velocity. The yield surface kinematically moves within the bounding surface which has isotropic/kinematic hardenings.

In visco-elasto-plastic case there are two time dependent and time independent deformation components, each of these components can further be divided into irreversible and reversible components [Chehab and Kim 2005, Chehab 2002, Drescher et al. 1993, Tashman 2003]. According to their report, the total deformation can be divided into four components: elastic, plastic, viscoplastic and viscoelastic. Among these, the plastic and viscoplastic components are irrecoverable while the elastic and viscoelastic components are recoverable. Further, the elastic and plastic components are time independent while viscoelastic and viscoplastic components are time dependent. The plastic and viscoplastic components generally are used to describe the rutting phenomenon in pavements.

It has been reported [Tashman 2003] that the total strain of HMA has been separated into four components as shown in eq. 4.36 .

$$
\varepsilon=\varepsilon^{\mathrm{el}}+\varepsilon^{\mathrm{pl}}+\varepsilon^{\mathrm{ve}}+\varepsilon^{\mathrm{vp}}
$$

Where $\varepsilon$ is the total strains, $\varepsilon^{\text {el }}$ is the elastic strain, which is recoverable and time and rateindependent, meaning that the material exhibits no permanent strains in a loading / unloading 
cycle and this state is independent of the rate of loading and unloading. $\varepsilon^{\mathrm{ve}}$ is the visco-elastic strain, which is recoverable and time-dependent. $\varepsilon^{\mathrm{pl}}$ is the plastic strain, which is irrecoverable and time independent. $\varepsilon^{\mathrm{vp}}$ is the visco-plastic strain, which is irrecoverable and time-dependent.
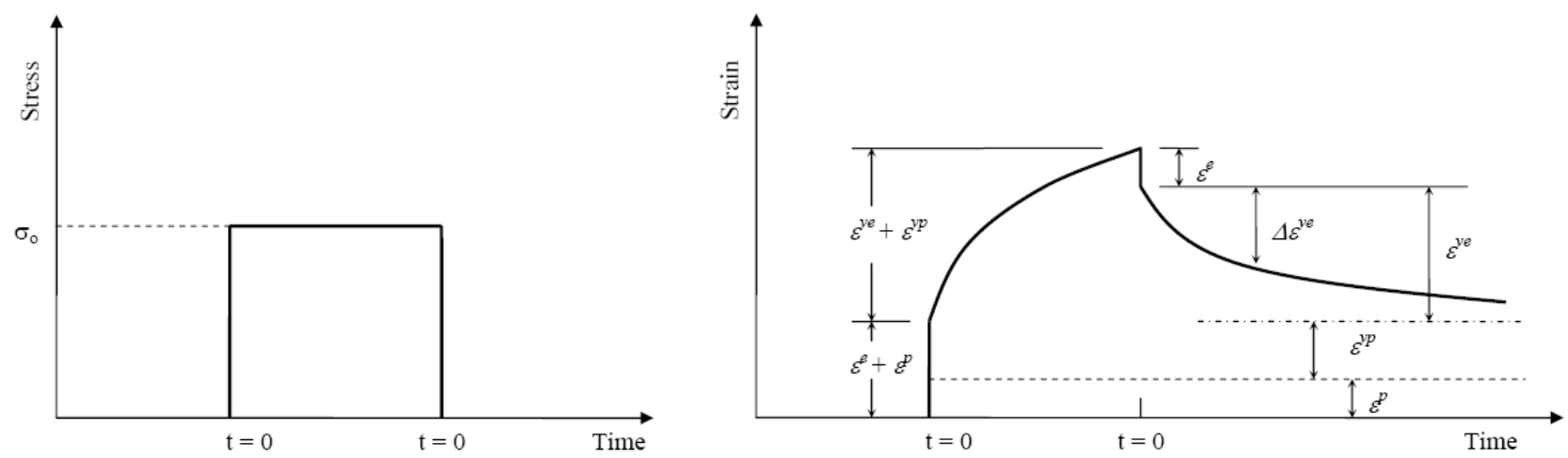

Figure 4.12 Schematic representation of strain components in an elasto-visco-plastic material [Tashman 2003]

On the other hand the viscoplasticity material model is expressed in equation 4.37 [Valoroso 2002]. As indicated in Figure 4.13, the model consists of a linear spring connected with a friction block in parallel with a dashpot whose presence introduces dependence of the response of from time.

$\varepsilon=\varepsilon^{\mathrm{el}}+\varepsilon^{\mathrm{vp}}$

Where $\varepsilon$ is the total strain, $\varepsilon^{\mathrm{el}}$ the elastic strain component and $\varepsilon^{\mathrm{vp}}$ is the visco-plastic strain component.

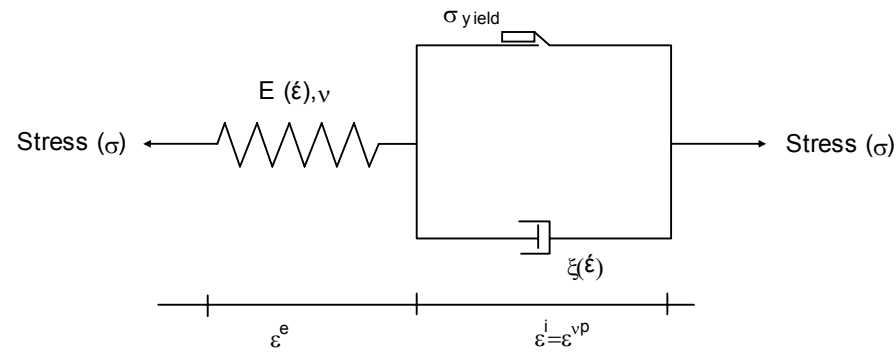

Figure 4.13 Material viscoelastoplastic model

In general, the viscous components depend on the load duration and the rate of loading/unloading. The instantaneous response reflects the time-independent component, whereas the viscous response reflects the time-dependent component. The relative contribution of each component depends on temperature and loading condition.

\subsubsection{Continuum Damage Mechanics Approach}

The Continuum Damage Mechanics is a branch of applied mechanics, which accounts for material deterioration caused by load application and environmental effects, prior to macro crack formation. Damage, in its mechanical sense in solid materials is the creation and growth of micro voids or microcracks which are discontinuous in a medium considered as continuous at a larger scale. 
During the past two decades damage mechanics has gradually become a systematic theory based on a general framework of thermodynamic and continuum mechanics. It has been applied to model creep, ductile plastic, brittle, fatigue and other damages [McShane and Roess 1990, Xiao et al. 1998]. CDM is characterized by the introduction of continuous damage variables that represents the local distribution of micro defects. The introduction of the damage variables requires the establishment of an additional equation, the so-called (damage) evolution equation, that expresses how the damage changes. The mechanical properties of materials depend on the damage state, which can be defined by the existing density, distribution and type of defects. Depending on their sizes and orientations, some of the micro defects will start growing under certain loading conditions. The practical utility of the selected damage variable depends on the description of the macroscopic effects. Once a suitable damage variable is chosen, the bases of scalar invariants of the independent variables, reflecting the symmetries characteristic of material can be determined.

For infinitesimal small deformation, it is convenient to adopt a micromechanical approach, in which the underlying physics of the failure mechanism is reflected. The damage state is characterized with the effective field theories, which determine the effects of micro damage systems on the materials behavior. Damage by the creation of free surfaces of discontinuities reduces the value of many properties:

- It decreases the elasticity modulus

- It decreases the yield stress before or after hardening

- It decreases the hardness

- It increases the creep strain rate

- It decreases the density

Some of these effects are used to evaluate the damage by inverse methods. Furthermore, the effects on mechanical strength and stiffness are different in tension and in compression due to micro cracks opening under tension and their closure under compression.

The continuum-damage model provides methods to develop constitutive equations within the concept of continuum mechanics to deal with the gradual degradation of materials during loading [Stigh 2006]. The premise of continuum damage mechanics is that by modeling the kinetics of damage accumulation, one can predict the duration of the tertiary creep stage and thus estimate the creep life [Rodin 2000]. It is also considered as an umbrella for many phenomenological and micromechanical models concerned with distributed damage. Continuum-damage mechanics is one of theories that has been used to model damage-induced behavior of asphalt mixtures [Kim et al. 2005]. It is hypothesized that phenomenologically determined internal state variables, which are determined from testing results, can be used to represent the overall locally averaged amount of damage in the sample. The continuum-damage models successfully predict structural degradation attributable to damage, but the models have limitations. Since the modeling parameters are determined from testing an asphalt mixture and the testing is based on an assumption that the sample is a statistically homogeneous continuum, each sample fabricated from different mixes of constituents requires its own constitutive tests and performance tests. The continuum-damage mechanics modeling is fairly mixture-specific and requires a new set of experiments to obtain the model parameters for each new mix. The asphalt mixtures generally exhibit complicated mechanical behavior and numerous damage modes. Precise identification of the damage-induced behavior of the asphalt mixtures is an appropriate way to meet design needs. Continuum-damage mechanics is one of theories that has been used to model damage-induced behavior of asphalt mixtures.

From the physical point of view, damage growth is a progressive internal deterioration of materials (voids, microcracks, deformations, etc.), which induces a loss of effective crosssectional area that carries load. According to the hypothesis of isotropic damage, the damage variable D [Pass 1990, Xiao et al. 1998] is defined by Kachanov equation as follows: 


$$
\mathrm{D}=\frac{\mathrm{A}-\tilde{\mathrm{A}}}{\mathrm{A}}
$$

And with the following values:

$\mathrm{D}=0$, corresponds to the undamaged state;

$\mathrm{D}=\mathrm{D}_{\mathrm{C}}<1$, corresponds to the failure state

Where D - damage variable

A - the overall cross-sectional area

$\widetilde{A}$ - the effective cross-sectional area which effectively bears the load.

$\mathrm{D}_{\mathrm{C}}$ - is called the critical value of damage variable.

The concept of effective stress is introduced to describe the effect of damage on strain behavior. Stress $\widetilde{\sigma}$ is written as the mean density of forces P acting on the effective cross-section:

$$
\widetilde{\sigma}=\frac{\mathrm{P}}{\widetilde{\mathrm{A}}}=\frac{\sigma}{1-\mathrm{D}}, \quad \text { where } \sigma=\frac{\mathrm{P}}{\mathrm{A}}
$$

On the other hand another interpretation of effective stress is given by introducing the concept of strain equivalence. The strain associated with a damaged state under applied stress is equivalent to the strain associated with an undamaged state under effective stress. Then, Hooke's law of a damaged material in the one-dimensional case can be written as

$$
\varepsilon=\frac{\widetilde{\sigma}}{\mathrm{E}_{0}}=\frac{\sigma}{\mathrm{E}_{0}(1-\mathrm{D})}
$$

Where $\varepsilon$ is the elastic strain and $\mathrm{E}_{0}$ the Young's modulus of undamaged material.

Let

$$
\mathrm{E}_{\mathrm{D}}=\mathrm{E}_{\mathrm{O}}(1-\mathrm{D})
$$

Where $E_{D}$ is the Young's modulus of damaged material; therefore [Pass 1990, Xiao et al. 1998] the damage is expressed as:

$$
\mathrm{D}=1-\frac{\mathrm{E}_{\mathrm{D}}}{\mathrm{E}_{\mathrm{O}}}
$$

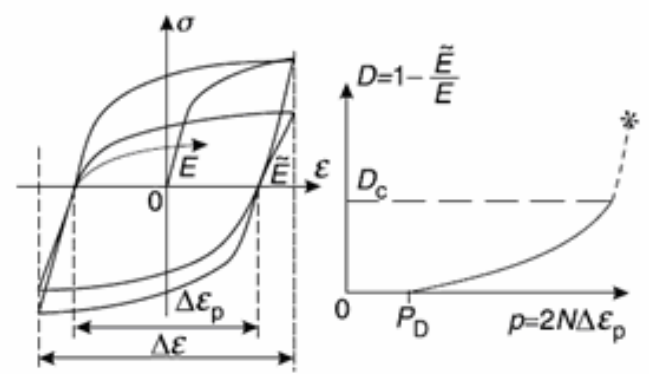

Figure 4.14 Measurement of damage by means of elasticity change [Lemaitre and Desmorat, 2005]

Damage is often non-isotropic due to micro cracking more or less perpendicular to the largest positive principal stress [Lemaitre and Desmorat, 2005]. Then, the surface density of micro defects in a plane with normal $\vec{n}$ acts through an operator which transforms the surface $A$ and $\overrightarrow{\mathrm{n}}$ of Fig. 4.15 into a smaller but continuous area, $\widetilde{\mathrm{A}}=\mathrm{A}-\mathrm{A}_{\mathrm{D}}$ and into another normal $\widetilde{\overrightarrow{\mathrm{n}}}$. To keep the same physical meaning as above the damage acts through the operator $(1-D)$ and

$$
\left(\delta_{\mathrm{ij}}-\mathrm{D}_{\mathrm{ij}}\right) \mathrm{n}_{\mathrm{j}} \mathrm{A}=\widetilde{\mathrm{n}}_{\mathrm{i}} \widetilde{\mathrm{A}},
$$

Where $\delta_{i j}$ is the Kronecker delta and D is a second order tensor. 
The damage $D$ is the operator which transforms the second order tensor $v_{i} n_{j} A$, of the reference configuration into the tensor of the effective configuration $v_{v_{i}} \widetilde{n}_{j} \widetilde{A}$. This is a fourth order tensor, where

$$
\left(I_{i j k l}-D_{i j k l}\right) v_{k} n_{j} A=v_{i} \widetilde{n}_{j} \widetilde{A}
$$

With the following symmetries:

$$
\mathrm{D}_{\mathrm{ijkl}}=\mathrm{D}_{\mathrm{ijlk}}=\mathrm{D}_{\mathrm{jikl}}=\mathrm{D}_{\mathrm{klij}}
$$

Another way to define a fourth order tensor D is to consider the operator which changes the initial elasticity tensor $\mathrm{E}_{\mathrm{ijkl}}$ into the actual elasticity tensor $\widetilde{\mathrm{E}}_{\mathrm{i} \mathrm{kl}}$ softened by damage:

$$
\left(\mathrm{I}_{\mathrm{ijrs}}-\mathrm{D}_{\mathrm{ijrs}}\right) \mathrm{E}_{\mathrm{rskl}}=\widetilde{\mathrm{E}}_{\mathrm{klij}}
$$

From a purely theoretical point of view, this definition does not yield a real state variable because it requires the knowledge of a particular behavior.
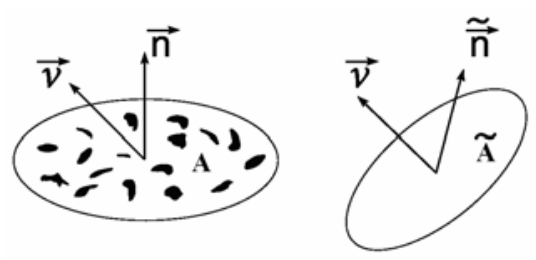

Figure 4.15 Schematic representation damage [Lemaitre and Desmorat, 2005]

\subsubsection{Fracture Mechanics and Cohesive-Zone Approach}

Fracture mechanics deals with the study of how a crack or flaw in a structure propagates under applied loads. It involves correlating analytical predictions of crack propagation and failure with experimental results. Cracks and flaws occur in many structures and components, sometimes leading to disastrous results. The engineering field of fracture mechanics was established to develop a basic understanding of such crack propagation problems. The analytical predictions are made by calculating fracture parameters such as stress intensity factors in the crack region, which can be used to estimate crack growth rate. Typically, the crack length increases with each application of some cyclic load, further, environmental conditions such as temperature or other climatic conditions can affect the fracture propensity of a given material.

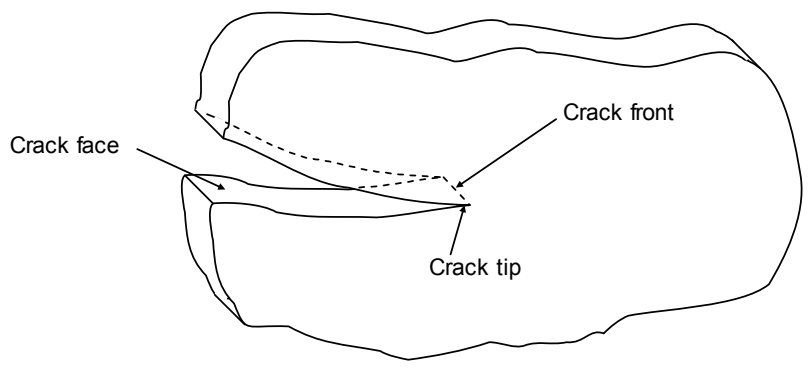

Figure 4.16 Representation of crack initiation and propagation

Fracture properties are considered as an essential requirement for asphalt pavements for which the prevailing failure mode is cracking due to low-temperature shrinkage stresses [Li and Marasteanu 2005]. Cracking can occur as a result of a single severe temperature drop (single event) or of multiple cycles of less severe temperature change (thermal fatigue). Low- 
temperature cracking is manifested as a set of surface-initiated transverse cracks of various lengths and widths. Although low temperature cracking has received attention for many years, only recently fracture mechanics has been applied to study the fracture properties of asphalt mixtures. The indirect tension test and semi circular bend (SCB) test are mainly used to determine the fracture properties as well as crack growth rate parameters to describe the fatigue cracking of asphalt mixtures of asphalt mixtures.
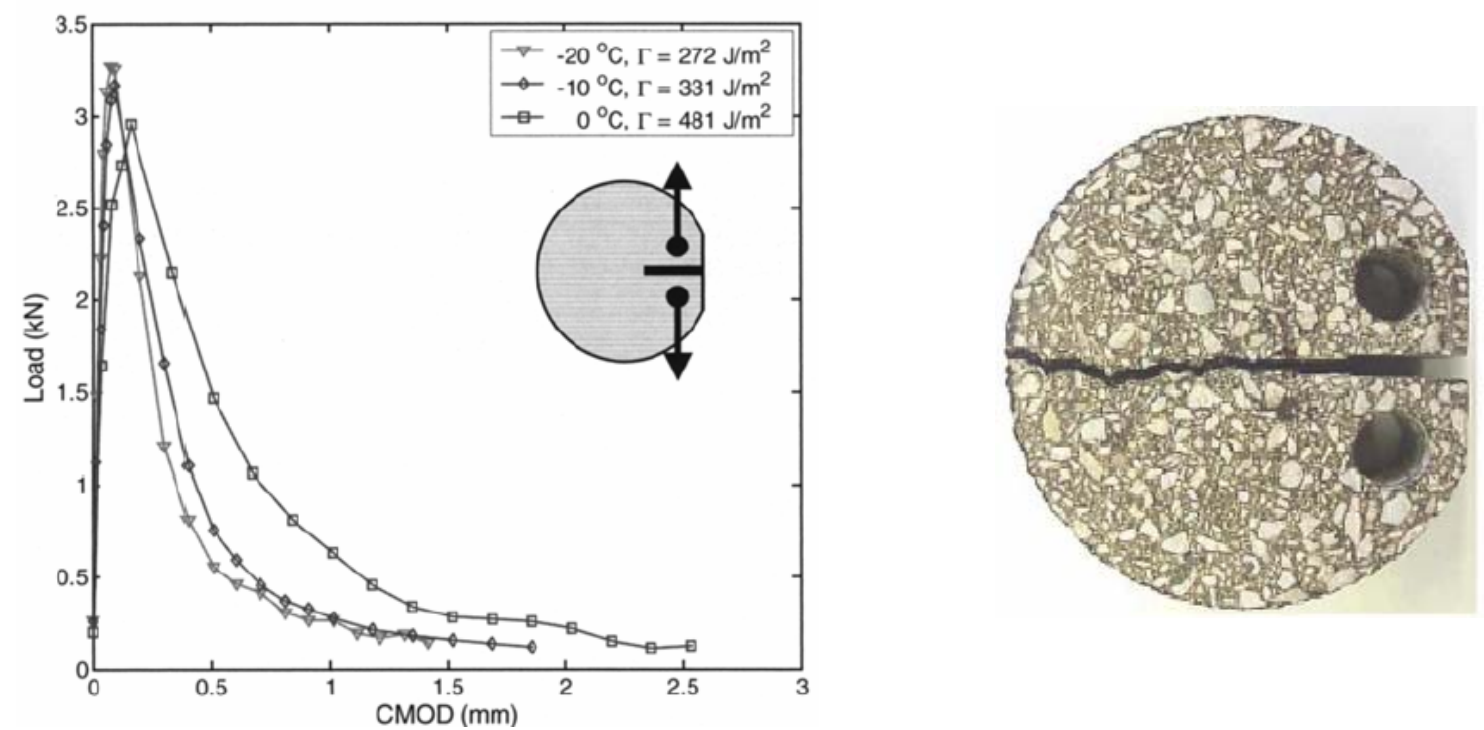

Figure 4.17 Fracture modelling and testing of asphalt concrete specimen [Wagoner et al. 2005]

\begin{tabular}{|c|c|c|}
\hline $\begin{array}{l}\text { Types of approaches } \\
\text { (1) }\end{array}$ & $\begin{array}{c}\text { Fracture mechanics } \\
\text { (2) }\end{array}$ & $\begin{array}{c}\text { Continuum damage mechanics } \\
\text { (3) }\end{array}$ \\
\hline Crack/damage driving force & $G=-\frac{\partial W}{\partial a}=$ Energy Release Rate & $f=-\frac{\partial W}{\partial S}=$ Thermodynamic Force \\
\hline Crack/damage resistance & $G_{c}=-\frac{\partial W_{c}}{\partial a}$ & $f_{e}=-\frac{\partial W_{s}}{\partial S}$ \\
\hline Damage evolution law for elastic body & $G=G_{c}$ & $f=f_{c}$ \\
\hline Damage evolution law for viscoelastic body & $\begin{array}{l}\dot{a}=C(G)^{n} \\
\dot{a}=A\left(J_{v}\right)^{k}\end{array}$ & $\dot{S}=A(f)^{a}$ \\
\hline
\end{tabular}

Table 4.1 Fracture mechanics versus continuum damage mechanics [Lee et al. 2000]

The cohesive-zone [Borst et al. 2004, Kregting 2005] approach has been adopted in various ways to incorporate the methodology in material's failure analysis. Failure in most engineering materials is preceded by the emergence of narrow zones of intense straining where the zone of deformation is highly strained and ultimately fractured and failed. A cohesive-zone is modeled by employing interface elements to predict the growth of the new boundary surface both internal and external to the body and the propagation of damage by incorporating the damage evolution law. When damage growth occurs these cohesive zone elements open in order to simulate damage initiation or growth. Since the damage path can only follow these elements, the direction of propagation strongly depends on the presence (or absence) of cohesive zone elements. The cohesive-zone constitutive model contains an internal state variable representing damage evolution within the cohesive zone. 


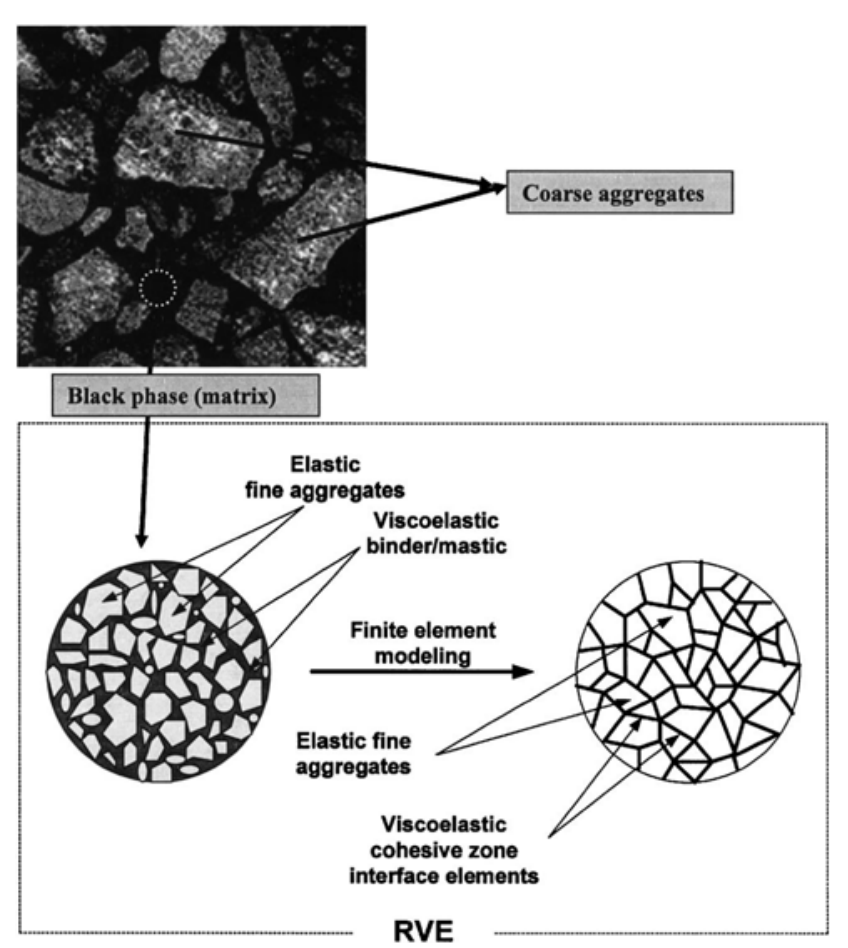

Figure 4.18 Representation of volume element in micro modeling [Kim et al. 2005]

\subsubsection{Micromechanics-Based Approach}

It is well known that asphalt concrete is a heterogeneous composite material. The distinct properties of aggregates and asphalt binder and their interface make the asphalt concrete a composite material with complicated stress-strain behavior. Individual movement of particles has been observed during loading of asphalt concrete. There are normal and shear forces between the aggregates and displacements (translation and rotation) of the aggregates. For this reason some researchers have questioned the validity of the continuum mechanics approach to represent the behavior of asphalt concrete and have argued in favor of micromechanical approach [Deshpande and Cebon 2004], which considers the properties of aggregates, the binder and the aggregate- binder interface separately. The aim of micromechanical approach is to find macrolevel state variables from micro- variables such as contact forces, grain displacements and local geometrical characteristics.

Therefore the micromechanics-based model can be considered as an alternative modeling approach. It predicts damage-induced mechanical response of asphalt mixtures. Heterogeneous geometric characteristics and inelastic mechanical behavior are taken into account by introducing finite element modeling techniques and a viscoelastic material model. The modeling also includes interface fracture to represent crack growth and damage evolution. The interface fracture is modeled by using a micromechanical nonlinear viscoelastic cohesive-zone constitutive relation. Fundamental material properties and fracture characteristics can be measured from laboratory tests and then incorporated into the model to predict rate-dependent viscoelastic damage behavior of the asphalt mixture.

\subsubsection{Dissipated Energy Approach}

The dissipated energy procedure provides an easy mechanistic means of examining the energy handling capability of a mixture as it relates to fatigue behavior in a manner not possible with traditional methods. In bituminous road pavement, a certain amount of work is done in deforming the surface layer during each cycle of traffic loading [Widyatmoko et al. 1999] in which part of this work is recovered in the elastic recovery of the surface layer, while the 
remaining work is dissipated. The dissipated work is exhibited by one or more damage mechanisms: fatigue crack initiation and propagation, permanent deformation (plastic flow) and heat.

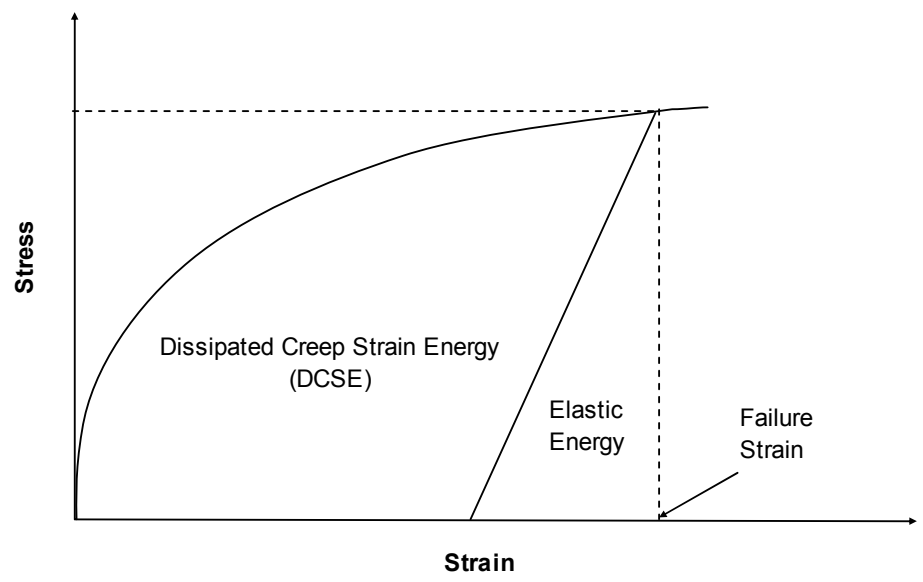

Figure 4.19 Dissipated creep strain energy determination

\section{Dissipated Creep Strain Energy (DCSE)}

The Dissipated Creep Strain Energy (DCSE) limit [Birgisson et al. 2007, Shen and Carpenter 2007, Widyatmoko et al. 1999] is one of the most important factors that control crack performance and hence durability in asphalt concrete mixtures. The dissipated creep strain energy threshold represents the energy that the mixture can tolerate before it fractures. DCSE is defined as the fracture energy, FE, minus the elastic energy, EE (Figure 4.19). The fracture energy is defined as the area under the stress-strain curve up to the point where the specimen begins to fracture. The elastic energy is the energy recovered after unloading the specimen. The failure strain $\left(\varepsilon_{\mathrm{f}}\right)$, tensile strength $\left(\mathrm{S}_{\mathrm{t}}\right)$ and fracture energy are determined from the indirect tensile (IT) strength test. From the resilient modulus test, the resilient modulus (MR) is obtained. The calculation of the DCSE was then determined as follows:

$\varepsilon_{0}=\left(\mathrm{M}_{\mathrm{R}} * \varepsilon_{\mathrm{f}}-\mathrm{S}_{\mathrm{t}}\right) / \mathrm{M}_{\mathrm{R}}$

$\mathrm{EE}=1 / 2 \mathrm{~S}_{\mathrm{t}}\left(\varepsilon_{\mathrm{f}}-\varepsilon_{0}\right)$

$\mathrm{DCSE}=\mathrm{FE}-\mathrm{EE}$

\section{Ratio of Dissipated Energy Change Approach}

Because of the nature of the traditional fatigue analysis and other existing fatigue analysis approaches, it is very difficult to study the hot mix asphalt (HMA) fatigue behavior at low damage levels. When using the traditional strain-fatigue life analysis approach, the design must rely on a large amount of laboratory fatigue testing, which is time consuming. At the low damage level, the fatigue life of tested samples will never reach failure (defined as the $50 \%$ initial flexural stiffness modulus reduction cycle) within a reasonable time frame, and the fatigue life has to be extrapolated based on long term fatigue tests, which is not convenient for either practical usage or research purposes. Recently the concept of ratio of dissipated energy change (RDEC) approach has been introduced for hot mix asphalt (HMA) material. Shen and Carpenter [2007] applied a new energy based approach to develop a simple procedure in order to predict the fatigue life of hot mix asphalt (HMA) airport pavements under low damage condition.

Damage only comes from the relative amount of energy dissipation due to each additional load cycle, while the energy dissipated through passive behaviors such as plastic dissipated energy and thermal energy is not considered [Shen and Carpenter 2007]. The incremental value of 
RDEC which can be calculated based on Equation 4.50, has a direct relation to damage accumulation. Definition of RDEC provides a true indication of the damage being done to the mixture from one cycle to another by comparing the previous cycle's energy level and determining how much of it contributed to damage [Shen and Carpenter 2007].

$$
R D E C_{a}=\frac{D E_{a}-D E_{b}}{D E_{a} \times(b-a)}
$$

Where $\mathrm{a}, \mathrm{b}$ - loading cycles a and $\mathrm{b}$, respectively

$$
\begin{aligned}
& \mathrm{RDEC}_{\mathrm{a}} \text { - the average ratio of dissipated energy change at cycle a, compared to cycle } \mathrm{b} \\
& \mathrm{DE}_{\mathrm{a}}, \mathrm{DE}_{\mathrm{b}} \text { - dissipated energy cycles a and } \mathrm{b} \text {, respectively, which are calculated directly by } \\
& \text { fatigue testing program, kpa }
\end{aligned}
$$

The typical RDEC vs. loading cycles curve can be divided into three stages. As shown in Figure 4.20, it develops a plateau (stage II) after the initial period (stage I). This plateau period, an indication of a period where there is a constant percentage of input energy being turned into damage, will extend throughout the main service life until a dramatic increase in RDEC occurs, which gives a sign of true fatigue failure (stage III). Here, the true fatigue failure represents final fracture with unstable crack propagation. It is a state where the material can no longer sustain further external loading due to internal fracture damage, and shows signs of macroscopically visible structural failure.

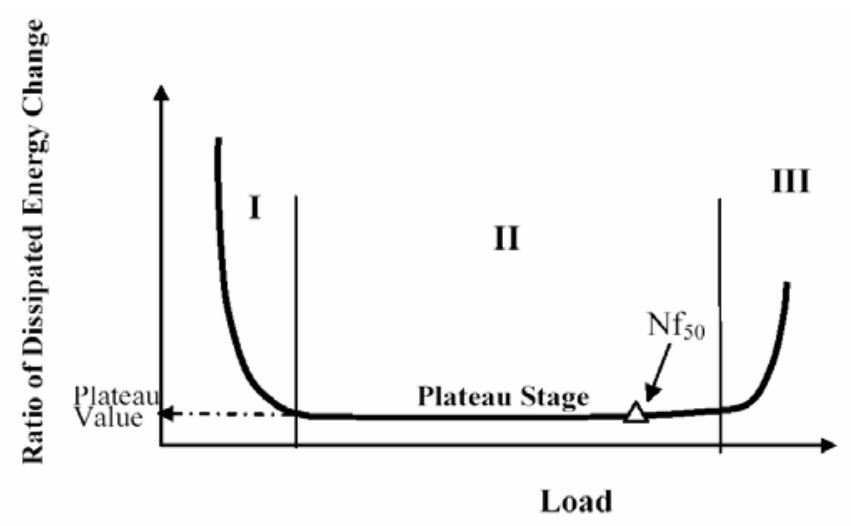

Figure 4.20 Typical RDEC vs. loading cycles plot and indication of PV

The RDEC value at the 50\% stiffness reduction point is defined as the plateau value, PV. This definition of PV is useful because the Nf50 value has been shown to relate precisely to the true fatigue failure (stage III). Accordingly there is a unique relationship between PV and Nf50 (fatigue life at $50 \%$ stiffness reduction point) for different mixtures, loading modes, loading levels, and testing conditions (frequency, rest periods, etc.). The established PV-Nf50 relationship is presented[Shen and Carpenter 2007] in Equation 4.51.

$$
P V=0.4428 N f_{50}^{-1.1102}
$$

The PV is a comprehensive damage index that contains the effect of both material property and loading effect, hence can be a fundamental energy parameter to represent HMA fatigue behavior. A low PV value can be found either in high fatigue resistant materials, low external loading amplitude, or both.

The RDEC approach defines a unique energy level for the energy based fatigue endurance limit PVL, which is the onset of the fundamental change in HMA fatigue behavior. If the energy level of a HMA mixture is below the PVL due to the combination effect of material resistance and external load, the mixture is expected to have extended long fatigue life. 


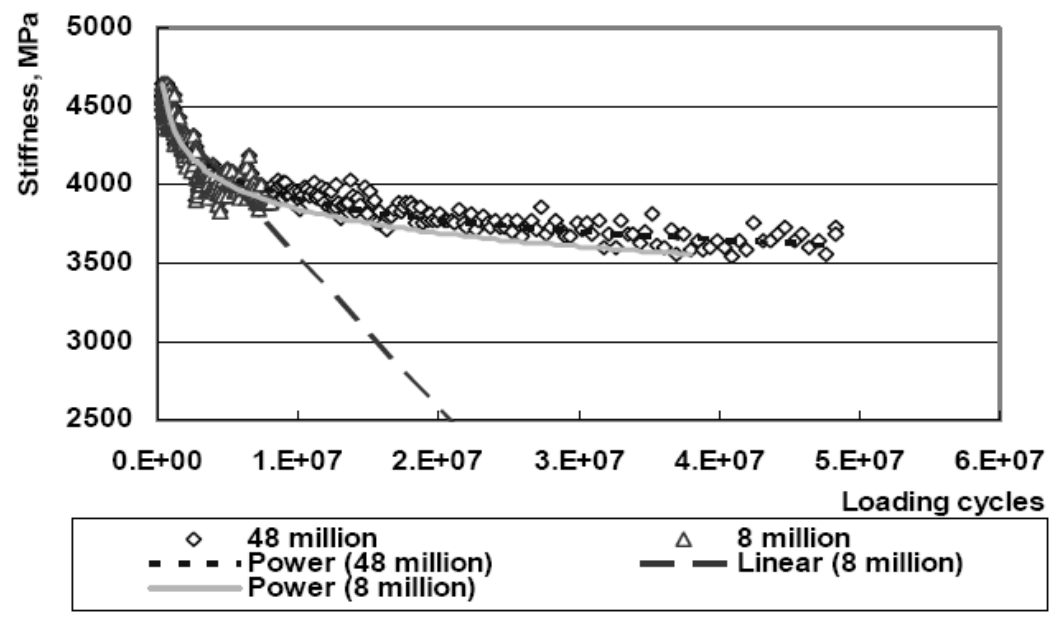

Figure 4.21 Stiffness vs. loading cycles curve [Shen and Carpenter 2007]

The use of energy dissipation has been extended for assessing the resistance to permanent deformation of bituminous mixtures [Widyatmok et al. 1998]. They explained that the energy dissipated by viscous flow created an accumulation of damage which could generate the creation of a plastic zone.

\subsubsection{Fatigue Endurance Limit Approach}

The "Fatigue Endurance Limit" (FEL) concept can also be used to limit the tensile strain or load damage at the bottom of asphalt layer below to reach extended fatigue life [Shen and Carpenter 2003 and 2007, Soltani et al. 2006]. These low tensile strain levels, typically well below 100 micro strains are used in the structural design through a linear extrapolation of the traditional fatigue data. It has long been felt that fatigue behavior at low strain levels does not follow the same relationship as the material subjected to strains at the normal levels. Indeed, it has even been postulated that there is a strain limit below which there is no fatigue damage. At such low strain/damage level, the damage accumulation in HMA mixtures is very slow and the load repetitions required to reach fatigue failure are extremely long. It is typical to design a flexible airport [Shen and Carpenter 2007] pavement with thick asphalt layer and low load response (tensile strain) at the bottom of asphalt layer. Detail description of the endurance limit and its application for asphalt concrete pavement is given under subtopic 4.3.

\subsection{Long Life Asphalt Design Concept}

Pavements are designed to carry the heaviest anticipated traffic both in terms of the total number of vehicles and, more importantly, the extremely high axle loadings applied by heavy vehicle (HV) under adverse climatic conditions without excessive distress and deformation. Frequent closures of roadways for the purpose of repairs and repaving constitute a growing problem for road administrations and road users, due to their costs, their limitations on road lane availability, the congestion and disruption they cause to traffic flows and the related delays and costs to road users. Long life pavements using advanced material and structural properties potentially have a great deal to offer high quality performance. In response to these challenges, many asphalt pavement industries are trying to develop a mechanism for heavy duty asphalt pavements that can serve for an extended period of time.

The primary purpose of the pavement is to reduce the stresses on the subgrade to such a level that the subgrade does not deform under the action of traffic. This can be achieved by providing strong and thicker asphalt concrete layers above the underground layers [Werkmeister et al. 
2003]. At the same time, the pavement layers themselves need to be strong enough to tolerate the stresses and strains to which each layer is exposed. Durable road pavement structure can be built by proper choice of construction materials, appropriate blending of each constituents and selection of mix properties with respect to stiffness modulus/strength, durability, flexibility as well as thickness of pavement layers which has the capability to perform well under adverse conditions without significant loss in stability during the intended service period. Longer design life for flexible pavements, which carry the heaviest volumes of traffic, will yield a lower whole life cost, less traffic delay as well as better economic growth for one nation. This requires strategies for design that decrease the need for maintenance and thereby cause less disruption to the road user. It will be beneficial for road sector to design and construct a reliable and durable road in order to save overall construction and operational costs. On this research, it is intended to develop a mechanism for asphalt concrete road that serves the highway community for extended period of time without the need for frequent maintenance and rehabilitation.

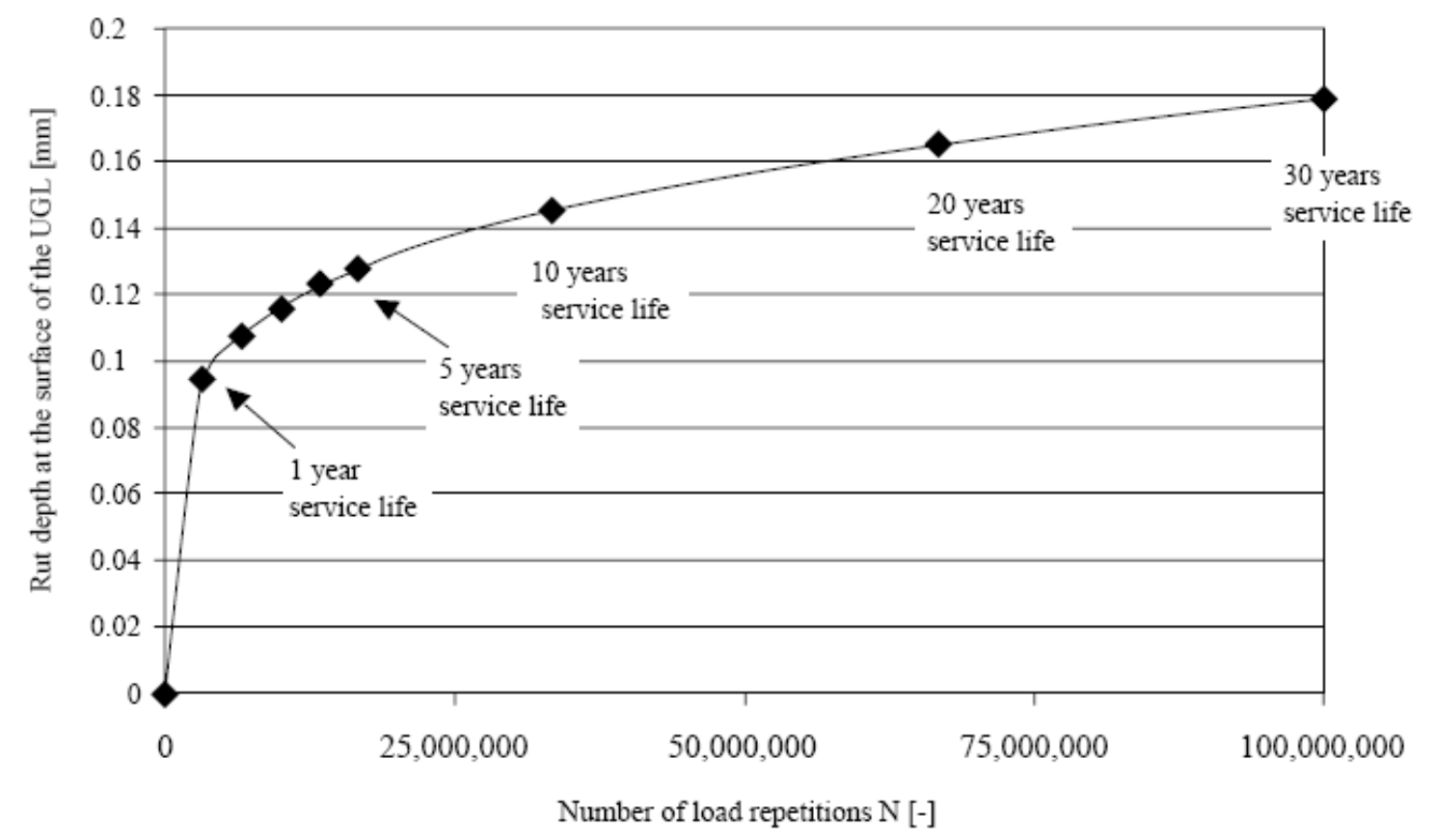

Figure 4.22 Rut depth at surface of the UGL as function of $\mathrm{N}$ (11.5 t-axle load); asphalt pavement, $340 \mathrm{~mm}$ [Werkmeister et al. 2003]

Until recently, it has been assumed that asphalt pavements should be designed for 20 years. In fact, some roads which had been constructed with the same design life have been serving for more than 40 years without the need for structural rehabilitation [Park et al. 2005]. In the near future the challenge for road engineers will be to design roads that require only minimal maintenance for a wide variety of conditions. In view of the rapid changes in materials, number and level of loads that currently occur; the presently used empirical strategies are not up to this challenge. Instead, a sound engineering approach of material response is needed.

The road is most vulnerable to damage from traffic when it is first laid, before its structural properties have had time to improve. Provided that the road is built strong enough initially, so that its main structural layers are not weakened by traffic loading, curing will progressively improve the load-spreading ability of these layers and make the road progressively less vulnerable to traffic-induced structural damage. This type of behavior implies that there is a minimum threshold strength above which the pavement should have a very long but 
indeterminate structural life. It is therefore reasonable to assume that the initial strength of the road should be a major factor in determining its future life.

The issue of prolonged service life of road pavements has been a key concern for road industries for the last several years. Long life pavements are seen as particularly desirable on heavily trafficked roads to avoid the costs of road maintenance works, including the delays they inflict on road users, particularly in congested traffic conditions. The long-life flexible pavement structures required to be designed for fatigue cracking originating at the bottom of the asphalt concrete layer and rutting of the asphalt and unbound layers. Good bonding between asphalt concrete layers has to be ensured to prevent critical tensile strains from occurring between lifts. They consist of but not limited to impermeable, durable, and wear resistant top layers; a stiff thick rut-resistant intermediate layer for structural strength; and a flexible fatigue-resistant bottom layer resting on a stable and high-strength foundation [Walubita et al. 2008]. The layer thicknesses vary depending on the traffic loading, environmental location, and materials/mixdesigns. However, the rut-resistant layer is often the thickest layer so as to provide sufficient load carrying capability.

Today, recent efforts in materials selection, mixture design, performance testing, and pavement design offer a methodology to obtain performances exceeding 50 years from asphalt pavement structures, while periodically replacing the pavement surface and recycling the old pavement material. Roads constructed to meet the demands of present-day traffic levels will need to be initially stronger to avoid excessive deterioration in their early life. The long life pavement design begins with a good foundation upon which to construct the thick asphalt layers. The asphalt layer has to be thick enough to resist bending so that cracks do not form at the bottom of the asphalt section. This layer can be made even more resistant to cracking either through the use of a little more asphalt cement in the mix, creating lower voids, or through the use of engineered binders in that layer, to preclude the cracks from starting. The intermediate layer of the structure has the quality of rut resistance. This layer can be designed to resist rutting, again through the use of high-quality aggregate and engineered binders. The top surface layer is a renewable surface that can be designed for specific applications. In some instances, the use of a conventional dense-graded mixture is adequate. In very high-traffic areas, the use of stone matrix asphalt (SMA) may be attractive, provided that the materials are available to construct it [Lane et al. 2008]. And in some places, engineers may want to use an open graded friction course (OGFC) on the surface, to reduce splash and spray and to provide better skid resistance during rainstorms. Both OGFC and SMA also have the advantage of absorbing road noise in addition to their strength.

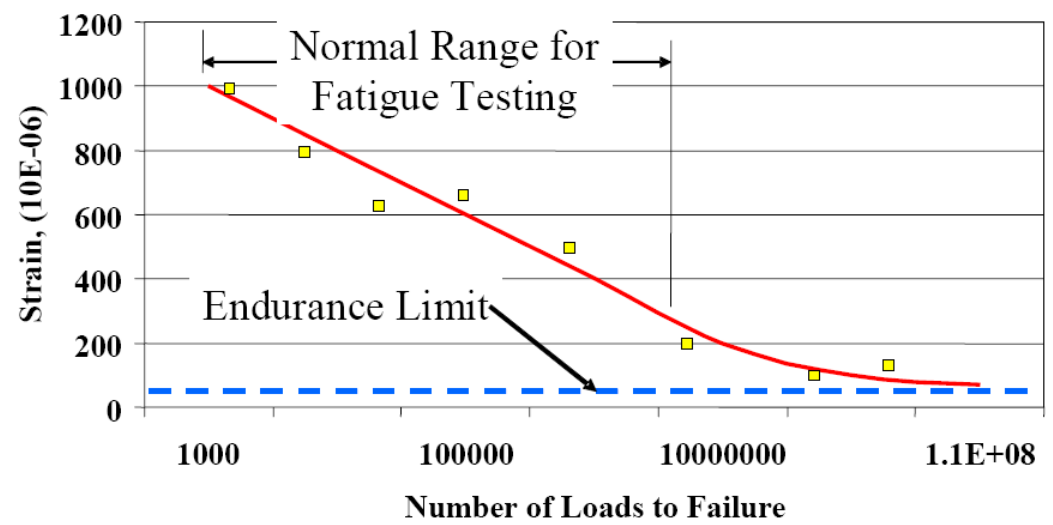

Figure 4.23 Normal fatigue testing results versus endurance limit testing [51]

It is a well-established concept in pavement design that decreasing the magnitude of the tensile strain [Soltani et al. 2006] at the bottom of the HMA results in an increase in pavement fatigue 
life. It is also believed by some asphalt and pavement design experts that an endurance limit exists for HMA, i.e. if the tensile strain is maintained at levels below the endurance limit, the pavement would have infinite fatigue life. The existence of a fatigue endurance limit has been postulated for a considerable time. With the increasing emphasis on extended-life hot-mix asphalt pavement (perpetual pavement) the verification of the existence of this endurance limit, a strain level below which none or very little fatigue damage develop, has become a substantial consideration in the design of these new multilayered full-depth pavements.

It has been found that high rates of rutting were associated with thin asphalt pavements [Nunn and Ferne 2001]. Sections of more than $200 \mathrm{~mm}$ tended to show a relatively slow rate of rutting. In pavements thicker than $200 \mathrm{~mm}$, the rutting was confined to the top layer of the pavement. As a result, only surface work was required to correct the problem. In thicker asphalt pavements, they also found that cracking did not start at the bottom and worked its way upward. Instead, it occurred mostly in the surface course and went down. They strengthened their argument that the elastic stiffness, and hence the load-spreading ability of asphalt road base, in thick, well-constructed roads increases steadily over time was an indication that traffic-associated deterioration of the road base did not occur. This improvement in load-spreading ability should have manifested as a reduction in deflection over the life of the road which implied that the overall stiffness of the pavements were increasing over time and that any traffic-related deterioration was more than offset by curing of the road base or strengthening of the foundation. They indicated (from their review of information on the performance of flexible roads in the United Kingdom) that, above a threshold strength, the road would remain structurally serviceable for a considerable period of time provided that nonstructural deterioration, in the form of surface-initiated cracks and deformation, has been detected and remedied before it could have a serious impact on the integrity of the road. To achieve a long life, they recommended that it would be also necessary for the road to be well constructed with good quality asphalt and a good foundation so that deterioration does not result from construction or material inadequacies.

The existence of long fatigue life was investigated under low-damaged condition [Shen and Carpenter 2005]. They concluded that there existed a threshold of the fatigue endurance limit, producing extremely long fatigue life, which could be applied as a mechanism to extended life pavement design. It was suggested [33] that the ability of heavy duty asphalt pavements to carry heavier axle loads was achieved by improving the stiffness of the asphalt structure and adjusting the thickness.

Park et al. [2005] attempted to develop a design procedure for determining the layer thickness for long-life pavement. It was emphasized that the performance of a pavement structure was strongly dependent on the pavement responses and strengths of the pavement layers. The critical pavement responses considered were tensile strain at the bottom of asphalt concrete layer for fatigue cracking $\left(\varepsilon_{\mathrm{t}}\right)$ and compressive strain on top of the subgrade for rutting $\left(\varepsilon_{\mathrm{c}}\right)$. The investigation indicated that the endurance limit for fatigue damaging at the bottom of AC layer was equal to or less than 65 ì strain. The compressive strain on top of the subgrade equal to or less than 200 ì strain was the endurance limit for permanent deformation. It was indicated that the strain levels, material stiffness and thickness of the layers influenced the endurance limit of the pavement. It was further suggested that by repairing and rehabilitating surface distresses periodically, the long-life pavement could capable of maintaining the pavement performance more than 40 years without any significant structural deficiencies or failures. In different parts of the world an effort is undergoing in this era to stretch the life of the pavement through mitigation [Epps 2000] of the source of distress or the use of more resistant materials.

A conservative calculation indicated [Nunn and Ferne 2001] that a road constructed with a thickness of more than $260 \mathrm{~mm}$ of asphalt would have a long but indeterminate life for traffic of up to $5 \mathrm{msa}$ per year and that $270 \mathrm{~mm}$ would be sufficient for any traffic loading. This higher thickness would give long life, even if curing did not take place, provided that the effective thickness of the asphalt layer was not reduced by deterioration due to cracking and concluded that the great majority of the thick pavements examined have maintained their strength or 
become stronger over time, rather than gradually weakening with trafficking as assumed in the current pavement assessment method based on deflection measurements. If the traffic growth rate is substantially greater, there may be economic advantage in designing the structural life of the road to last much longer. Nun and Ferne further indicated that pavements with less than about $180 \mathrm{~mm}$ of asphalt material deform at a high rate, but thicker pavements deform at a rate about two orders of magnitude less; the sudden transition suggested a threshold effect. Their results suggested that for these thicker pavements nearly all the rutting were due to deformation within the upper layers and that the traffic-induced strains in the subgrade are too low to cause structural deformation. It was apparent that below thicknesses of about $180 \mathrm{~mm}$, the much higher traffic-induced subgrade strains had a much greater effect.

A research study was conducted by Soltani et al. [2006] on hot-mix asphalt concrete using a new uniaxial fatigue testing protocol indicated the existence of an endurance limit. The study included testing of four different mixtures. In the study, cylindrical specimens of $120 \mathrm{~mm}$ in height and $80 \mathrm{~mm}$ in diameter were tested by applying a tension-compression loading at $10{ }^{\circ} \mathrm{C}$ and $10 \mathrm{~Hz}$. Each test included millions of cycles of sinusoidal loading with strain amplitudes of 20 and $30 \mu$ strain. They proposed a new fatigue testing protocol in which three stages of continuous loading without any rest period were considered. The same strain level, not exceeding the endurance limit of the HMA and consequently at a level that did not produce fatigue damage, was applied during Stages I and III. During Stage II, a strain with a magnitude exceeding the endurance limit and consequently causing fatigue damage was applied.

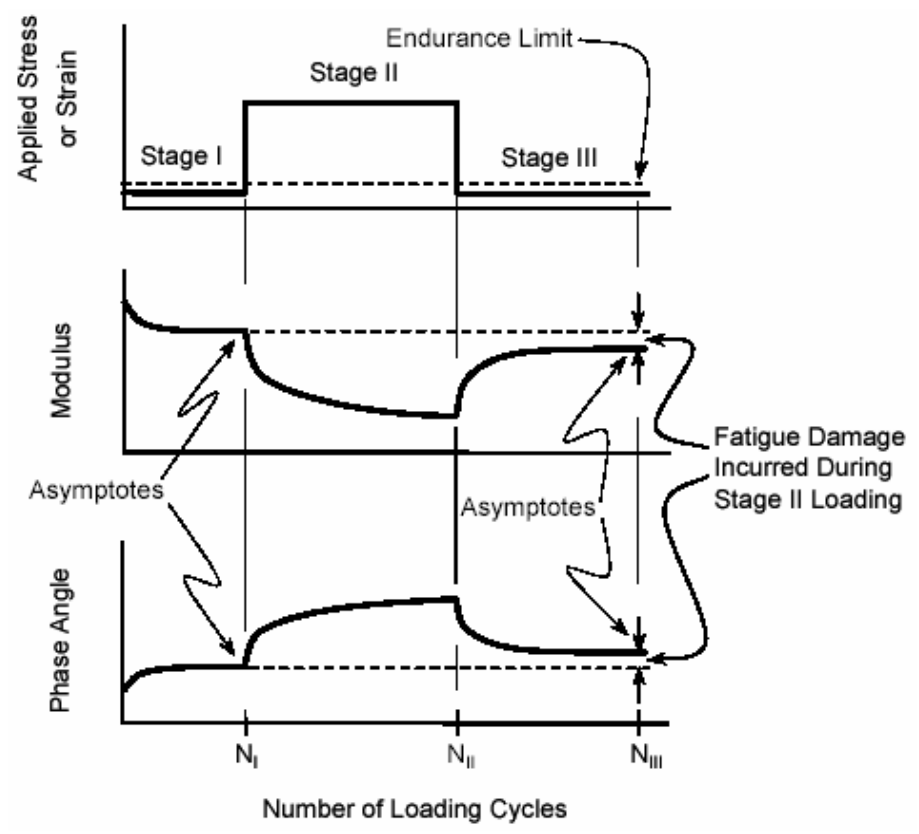

Figure 4.24 Schematic of loading in Stages I, II, and III [Soltani et al. 2006]

Under the testing procedure followed during their research some specimens had shown no fatigue damage after millions of cycles of loading when subjected to strain amplitudes of 30 $\mu$ strain. These results suggested that an endurance limit existed for at least some mixtures and it was at least $30 \mu$ strain at $10{ }^{\circ} \mathrm{C}$. The primary criterion in deciding whether such a limit exists was the difference between the magnitude of modulus at the end of Stage I and at the end of Stage III.

The test results had indicated that three of the mixtures tested exhibited an endurance limit below which no fatigue damage was expected regardless of the number of loading cycles applied. Fatigue data are presented that were collected on a surface mix and a binder mixture tested for an extended period from 5 million to 48 million load repetitions at strain levels down to 70 microstrain [Soltani et al. 2006]. The fatigue results are analyzed in the traditional manner and 
using the dissipated energy ratio. This analysis shows that there is a difference in the data at normal strain levels recommended for fatigue testing and at the low strain levels. This difference cannot substantiate an endurance limit using traditional analysis procedures, but the dissipated energy approach clearly shows a distinct change in material behavior at low flexural strain levels, which supports the fact that at low strain levels the damage accumulated from each load cycle is disproportionately less than what is predicted from extrapolations of fatigue testing at normal strain levels. This reduced damage may be attributed to the healing process. The conclusion of the study was that laboratory testing could verify the existence of a fatigue endurance limit in the range of 90 to 70 microstrain below which the fatigue life of the mixture was significantly extended relative to normal design considerations.

Laboratory and full scale performance tests were conducted by Lee et al [2007] for a high modulus asphalt binder (HMAB) and mixes (HMAM) for long life asphalt pavements. Various laboratory tests had been conducted on HMAB and HMAM to evaluate the performance characteristics such as fatigue cracking and permanent deformation. In addition, a full scale performance testing had been performed to evaluate the structural capacity and pavement performance of long life asphalt pavements. In their study, a HMAB was developed using the high boiling point petroleum and polymer. The high boiling point petroleum was first added to the conventional asphalt binder to increase the binder stiffness. As a result, the high boiling point petroleum successfully affected the increase of the binder stiffness but made the binder more brittle. To minimize the cracking potential due to the brittleness and increase rutting resistance of the binder, a $4 \%$ of styrene-butadiene-styrene (SBS) polymer was added. A liquid type of anti stripping agent has also been added to binder to minimize the moisture induced damage.

\begin{tabular}{llllll}
\hline Property & PG 64-22 & PMA & HMAB & $\begin{array}{l}\text { French hard grade } \\
\text { asphalt }\end{array}$ \\
\cline { 5 - 6 } & & & & $35 / 50^{\mathrm{a}}$ & $10 / 20^{\mathrm{b}}$ \\
\hline $\begin{array}{l}\text { Penetration, } \\
25^{\circ} \mathrm{C}(\mathrm{dmm})\end{array}$ & 70 & 63 & 28 & $35 / 50$ & $10 / 20$ \\
$\begin{array}{l}\text { Softening } \\
\text { point }\left({ }^{\circ} \mathrm{C}\right)\end{array}$ & 48 & 100.3 & 89 & & $62-70$ \\
$\begin{array}{l}\text { Fraass temperature } \\
\left({ }^{\circ} \mathrm{C}\right)\end{array}$ & -11 & -18 & -8 & & +3 to -8 \\
$G^{\mathrm{a}}, 15{ }^{\circ} \mathrm{C}(\mathrm{MPa})$ & 7.1 & 7 & 21 & 34.5 & $40-90$ \\
\hline $\begin{array}{l}\mathrm{a} \\
\mathrm{b} 35-50 \mathrm{dmm} \text { of penetration. }\end{array}$ & & & & \\
$\mathrm{b} \quad 10-20 \mathrm{dmm}$ of penetration.
\end{tabular}

Table 4.2 Comparison of asphalt binder properties [Lee et al. 2007]

It had been indicated that the dynamic modulus of the HMAM was higher than the conventional mix especially at low frequencies (According to the time temperature superposition principle, the low frequency corresponds to the high temperature), which might have been due to the effects of high boiling point petroleum and polymer in the mix. Dynamic modulus test results showed that the modulus of the HMAM was 50\% more than those of the conventional mix at high temperatures. The increase in the modulus of the HMAM was mainly due to the effects of the high boiling point petroleum and polymer.

The wheel tracking test was conducted on the specimens $60{ }^{\circ} \mathrm{C}$ to evaluate the permanent deformation characteristics of asphalt mixtures. Comparisons showed that the conventional mix has a maximum rut depth of about $8 \mathrm{~mm}$ at 20,000 cycles. On the other hand, the maximum rut depth of the HMAM is about $2.5 \mathrm{~mm}$ and there was no increase in the rut depth after 5000 of load applications, which showed that the HMAM had a great potential to reduce the permanent deformation. The wheel tracking test results indicated that the resistance to permanent deformation of the HMAM was two times higher than the conventional mix. It was also observed 
that the fatigue lives of the both mixes had increased with the application of rest period. The increase in fatigue lives could be due to micro-damage healing occurred during the rest period. They presumed that the more increased in fatigue lives of the HMAM compared to the conventional mix were observed with rest period representing a better healing potential of the HMAM. The SBS polymer mixed in the HMAB could be the main source of better healing potential. For the fatigue test results with rest period, the resistance to fatigue cracking of the HMAM seemed to be better than that of conventional mix where the tensile strain value was lower than $150 \mu$ strains. For typically thick asphalt pavements, the tensile strain values at the bottom of asphalt layer were lower than $150 \mu$ strains. Thus, it could be said that the fatigue resistance of the HMAM was better than that of the conventional mix for the thick asphalt pavements. On the other hand, the fatigue resistance of the HMAM was worse than that of the conventional mix for thin asphalt pavements because of the increased stiffness of the HMAM compared to the conventional mix.

Accelerated Pavement testing (APT) was conducted [Lee et al. 2007] at room temperature of $25^{\circ} \mathrm{C}$ showed that HMAM pavement had smaller tensile strain values at the bottom of the asphalt layer when compared with the conventional mix. It was also found that the HMAM had about 510 times better resistance to fatigue cracking than the conventional mix where the tensile strain level was less than 150 micro strain. Further to compare the rut resistance of the conventional and HMAM pavements, the accelerated pavement testing with 6.2 ton of load level has been performed on the thick pavement sections. The temperature at a depth of $5 \mathrm{~cm}$ below the surface was maintained at $50{ }^{\circ} \mathrm{C}$ during the testing and plotted as shown below. The results from APT tests indicated that the rut depth of the conventional mix and the HMAM section were 5.3 and $3.0 \mathrm{~mm}$, respectively after 90000 of load application.

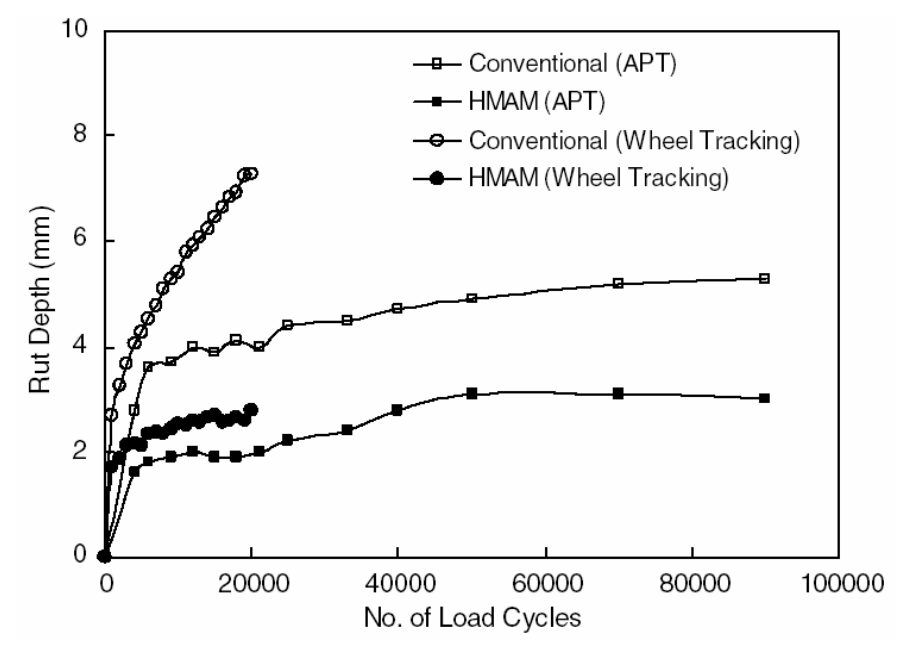

Figure 4.25 Comparison of rut depths for conventional mixtures and HMAM pavement [Lee et al. 2007]

It had been suggested to use the high modulus [Lee et al. 2007] asphalt (hard grade asphalt binder) mixes in base layer rather than increasing the base thickness. The results of performance test indicated that the resistances of the HMAM against moisture, rutting, and fatigue damage were better than those of the conventional mix. The low voids content and high stiffness of HMAC provides protection to the base course and great resistance to rutting [Corté 2001].

A fatigue endurance limit has also been postulated [Shen and Carpenter 2005] to exist in hotmix asphalt pavement performance using dissipated energy concept. The ratio of dissipated energy change succeeds in defining and investigating the existence of a fatigue endurance limit with a unique relationship between plateau value (PV) and fatigue life (Nf), regardless of straindamage levels, mixture types, loading modes, and other testing conditions. By applying the validated relationship between PV and Nf, the extremely long fatigue life under low straindamage condition can be predicted without performing millions of loading cycles. 
The concept of the existence of a fundamental crack growth threshold is based on the observation that micro-damage (i.e., damage not associated with crack initiation or crack growth) appears to be fully healable, while macro-damage (i.e., damage associated with crack initiation or growth) does not appear to be healable. This indicates that a damage threshold exists below which damage is fully healable. Therefore, the threshold defines the development of macro-cracks, at any time during either crack initiation or propagation, at any point in the mixture. If loading and healing conditions are such that the induced energy does not exceed the mixture threshold, then the mixture may never crack, regardless of the number of loads applied.

It has been determined that the dissipated creep strain energy (DCSE) limit and the fracture energy (FE) limit of asphalt mixtures suitably define the lower and the upper threshold values. These parameters can easily be determined from the stress-strain response of a tensile strength test, as shown in Fig. 4.19. The fracture energy limit is determined as the area under the stressstrain curve, while the dissipated creep strain energy limit is the fracture energy minus the elastic energy at the time of fracture.

Recent work was made on the development of a viscoelastic fracture mechanics-based pavement cracking model with the concept of the existence of a fundamental crack growth threshold [Birgisson et al. 2007] as the key element in defining the cracking mechanism and fracture resistance of asphalt mixtures. The concept is based on the observation that if the strain energy threshold is not exceeded, micro-damage (i.e., damage not associated with crack initiation or crack growth) appears to be fully healable, whereas when the threshold is exceeded not healable macro-damage (i.e., damage associated with crack initiation or growth) occurs. Fracture (crack initiation or crack growth) can develop in asphalt mixtures in two distinct ways, defined by two distinct thresholds. The lower threshold is associated with continuous repeated loading. When cyclic stresses significantly below the tensile strength occur, cracking will eventually occur if the rate of damage accumulation exceeds the rate of healing during the loading period. In contrast, the upper energy threshold corresponds to that threshold required to fracture the mixture with a single load application. In this case, fracture would occur if any single load applied during the loading cycle exceeds the threshold required to fracture the mixture with a single load application. Essentially, fracture would not occur during a single load application unless the upper threshold is exceeded, even when the lower threshold is exceeded.

A verification of fracture energy density as a fundamental fracture threshold in hot mix asphalt was presented [Birgisson et al. 2007]. Fracture energy density was evaluated from both the Semi-Circular Bending Test (SCB) and the Three Point Bending Beam Test (3PB). Their experimental analyses were enhanced by a Digital Image Correlation System. The system located the specific point(s) at which cracks initiated and propagated. Also it allowed for the definition of a full strain field over a finite area on the surface of the test specimen, using displacement measurements in the sub-micron range. They concluded that using rigorous interpretation of test conditions and appropriate analysis techniques for identification of fracture initiation, the same fracture energy density and tensile strength at fracture were obtained from the Superpave Indirect Test, the Semi-Circular Bending Test, and the Three Point Bending Beam Test for both unmodified and heavily polymer modified mixtures.

\subsection{Probabilistic Approach}

"Due to many different factors, such as simplified assumptions made for characterizing the behavior of pavement structures and the variability associated with material properties, the performance of a pavement can never be predicted with an absolute certainty; at best, the performance can be predicted only with the associated probability. Failing to recognize such a fact can often lead to improper design and management decisions" [Zhang and Damnjanovi, 2006]. Uncertainty of a pavement structural system arise due to the inherent irregularities in the properties and behavior of the system, lack of knowledge about the system's behavior and 
uncertainty in the occurrence of both acknowledged and unacknowledged errors. The most common sources of variability contributing to the response of the pavement structure

a. Variability in current, projected traffic characteristics and associated parameters such load, contact pressure, speed and other vehicular characteristics

b. Material variations or the natural variations associated with the properties of materials used for construction

c. Pavement performance related variability as of surface and /or structural deterioration, quality control and assurances techniques

d. Variability from construction activity, the variation of as built thicknesses of pavement layers and quantification of mismatches that occur between design and construction

e. Environmental variations and other miscellaneous variations.

The use of the probability techniques to characterize the variability of the design parameters would enable an engineer to design a pavement more effectively for a particular application. The tool would allow evaluating the effect of different degrees of variability in a pavement system. Design standards are based on the reliability and/or probability concept that a particular set of distresses or distress manifestations will remain below or within the permissible limit and the pavement performs satisfactorily during the design life under the prevailing road way, traffic and environmental conditions. The selection of a certain level of reliability a particular system primarily depends on the projected usage and the associated risk on that system. 


\section{Thermal Behavior of Asphalt Concrete Pavement}

A significant factor that affects the performance and life span of a pavement is the influence of temperature. It contributes to certain common types of asphalt pavement distresses such as permanent deformation, bleeding (associated with high temperature environments) and thermal cracking (associated with low temperature environments). It has also been recognized that the effect of temperature is an important design parameter in pavement engineering.

At low-temperature zone the thermal cracking is the prevailing failure mode. During thermal cooling, asphalt stiffness increases continuously which results in higher stresses for a given shrinkage strain. Within the intermediate temperature zone, asphalt concretes are generally harder and more elastic than at higher temperatures. The prevailing failure mode at these temperatures is fatigue damage. At high temperature, asphalt concretes get softer therefore the main distress mechanism becomes rutting.

The actual temperature of the pavement varies along the pavement depth and with time which makes an ever-changing temperature field [Huang et al. 2008]. Simultaneously the asphalt mixtures serving as the primary pavement material always show a viscoelastic behavior and they are easily subjected to the influence exerted by the temperature fluctuation.

Many roads experience extreme daily low temperatures during the winter and high temperatures and solar radiation during the summer. These conditions alone or in combination with large daily temperature fluctuations may cause thermal cracking problems in asphalt concrete pavements. There are two main forms of thermal cracking resulting from either a single drop in temperature to an extreme low below the fracture temperature of the asphalt-aggregate mixture which is referred as low temperature cracking or from a series of repeated temperature fluctuations with temperatures above the fracture temperature of the asphaltaggregate mixture identified as thermal fatigue. Thermal cracking induced by sharp and rapid drops in pavement temperature causes extreme thermal contraction and fracture that appears as regularly spaced transverse cracks across the complete asphalt concrete surface. Such crack permits water infiltration into the underlying pavement layers that can cause structural failure of the pavement. It also contributes directly to a loss of smoothness.

\subsection{Thermal Response of Pavement Structure}

Structural components or systems subjected to diurnal temperature cycling are often prone to a deterioration mechanism commonly known as rutting at high temperature and as thermal cracking, particularly when significant restraints against contraction are present.

The amount of stress that builds up when an asphalt pavement cools and tries to shrink depends on the thermal coefficients of the materials, the amount of friction with the subbase and hence the level of confinement, the temperature change, the ability of the system to relieve stress, and the stiffness of the materials. In regions that experience large daily temperature variations or extremely low temperatures, the thermal conditions plays a major role in the reflective cracking response of a pavement. On one hand, binder properties (stiffness, ageing, penetration grade) are sensitive to temperature variations. On the other hand the combination of wheel loads and a rapid decreasing of temperature have been the most likely causes of high states of stress/strain and responsible by the reflective cracking phenomena.

The temperature distribution in an asphaltic pavement is directly affected by the thermal environmental conditions to which it is exposed. The primary modes of heat transfer [Yavuzturk et al. 2005] are incident solar radiation, thermal and long-wave radiation between the pavement surface and the sun as shown in Figure 5. 1. The intensity of solar radiation is dependent on diurnal cycles, the latitude, and the incident angle between the surface and sun's rays. The ability to accurately predict asphalt pavement temperature at different depths and locations based on thermal environmental conditions greatly help pavement engineers in 
performing back-calculations of pavement modulus values and in selecting the asphalt concrete properties to be used in various pavement layers.

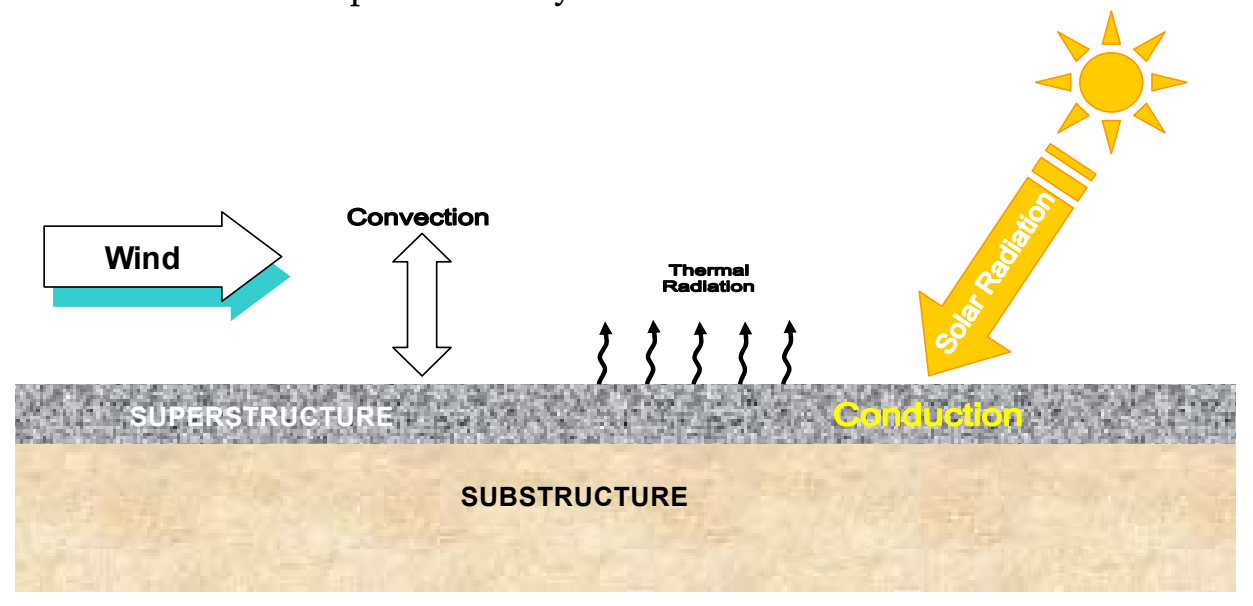

Figure 5.1 Energy balance on asphalt concrete pavement surface

The volumetric heat capacity of the pavement thermal mass strongly influences the thermal behavior of the pavement where heat transfer is dominated by conduction. On the other hand, the minimum asphalt temperature at the pavement surface is primarily dependent on the ambient wind conditions since convective cooling effects dominate the heat transfer at the surface. Theoretically, the higher the wind speed is, the higher is the convection heat transfer coefficient at the asphalt surface, resulting in lower surface temperatures. A significant amount of energy received by the asphalt is due to incident solar radiation although some convective heat gains occur at times when the ambient air temperature is greater than the asphalt surface temperature. As the volumetric specific heat capacity of the asphalt mix is increased the predicted asphalt temperatures decrease because asphalt mixes with higher volumetric specific heat capacities can store more energy, resulting in lower temperatures.

Daily temperature variations have an important influence in the pavement thermal state a few centimeters below the surface. The differential maximum asphalt temperature variation at a depth of $20 \mathrm{~mm}$ is significantly less than at the surface because heat transfer inside the asphalt mix is only due to conduction whereas convective cooling dominates at the surface, resulting in higher heat transfer rates and larger temperature fluctuations [Yavuzturk et al. 2005]. Due to the thermal mass of the asphalt a slight time shift is observed as to when the maximum temperature is reached at varying values of thermal conductivity. This time shift is not observed at the asphalt surface since the energy balance boundary condition assumes an infinitesimally small control volume without a thermal mass.

Thermal environmental conditions, to which pavements [Yavuzturk et al. 2005] are exposed, significantly impact pavement stability and long-term performance. Accurate prediction of the temperature profile in pavements greatly aids pavement engineers in the design process. Specifically, it allows for the assessment of pavement deflection, back-calculation of time and temperature dependent pavement modulus values, estimation of frost action and frost penetration as well as thaw onset, calculation of the cooling rates for freshly laid asphalt layers, and assessment of diurnal and seasonal heating and cooling effects. In order to calculate pavement thermal response, it requires evaluating the temperature distribution in bituminous layers during a typical twenty-four hours period.

The air temperature reaches the highest value at 14:00 [Huang et al. 2008]. Due to heat exchange, the change of the pavement temperature is delayed compared with air temperature; the deeper the location is the later each portion of pavement reaches its highest temperature. 


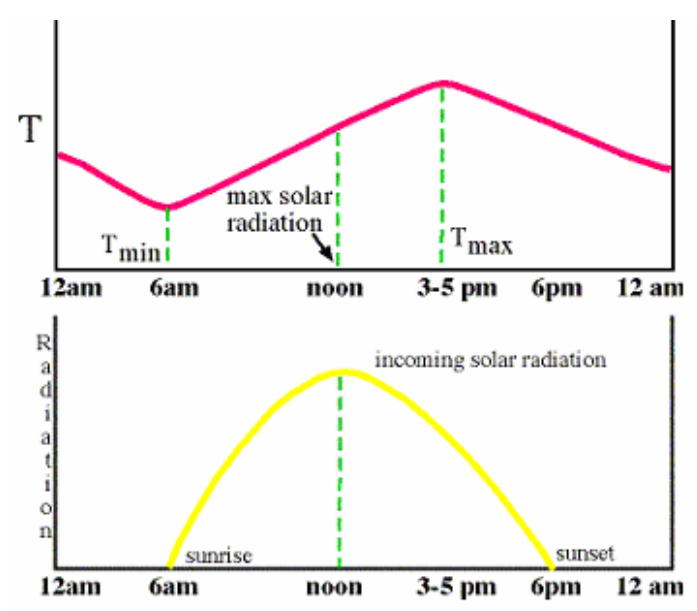

Figure 5.2 Time lag between the maximum temperature of pavement layer and air [47]

The temperature change is much higher inside the asphalt mix than at the surface due to the nothermal mass boundary condition at the asphalt surface. Because of the thermal mass of the asphalt mix, a significant time shift of about $3 \mathrm{hrs}$ is observed in the maximum temperature predictions between the maximum and minimum volumetric heat capacities considered.

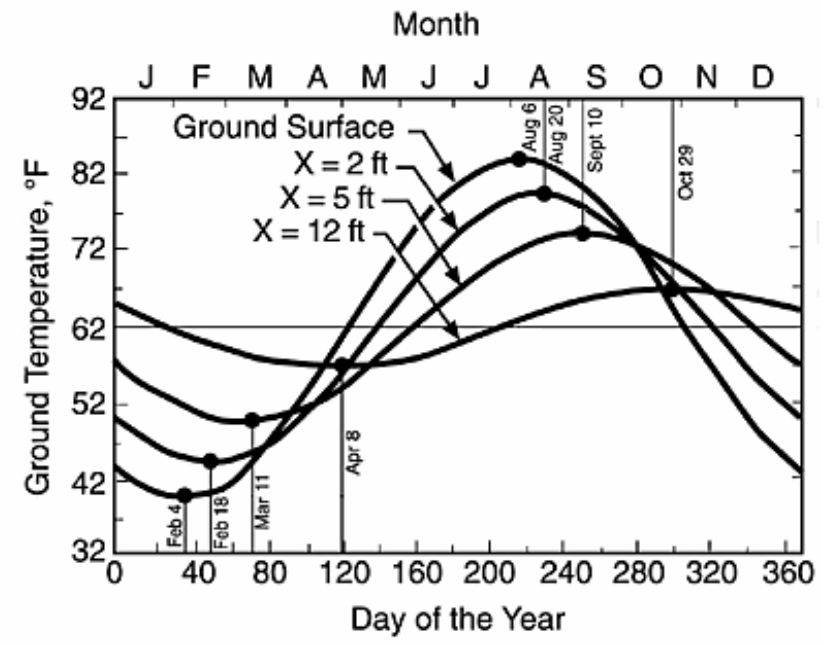

Figure 5.3 Annual temperature fluctuations in asphalt pavements [52]

The maximum asphalt surface temperature predictions equations are based on the energy balance concept. The calibration of the equations involves identifying the best fit of values for the asphalt surface absorptivity, the transmission coefficient of air, the emissivity of air and the asphalt surface, the asphalt surface heat transfer coefficient and the conductivity of the asphalt material. A general model used to predict the pavement temperature is expressed [Diefenderfer et al. 2006] as follows

$T_{p}=a+b T_{a}+c R_{s}+d P_{d}$

Where $\mathrm{T}_{\mathrm{p}}$-predicted pavement temperature $\left({ }^{\circ} \mathrm{C}\right)$, a-intercept coefficient, b-ambient temperature coefficient, $\mathrm{T}_{\mathrm{a}}$-measured ambient temperature $\left({ }^{\circ} \mathrm{C}\right)$, c-calculated solar radiation coefficient, $\mathrm{R}_{\mathrm{s}}$-alculated solar radiation $\left(\mathrm{kJ} / \mathrm{m}^{2}\right.$ day), d-depth coefficient, and 


$$
\mathrm{P}_{\mathrm{d}} \text {-depth within pavement }(\mathrm{m}) \text {. }
$$

Superpave binder specification is based directly on the climate in which the pavement will serve. The specification provides a correlation between climatic conditions at a given location and pavement performance [Hassan et al. 2008, Yavuzturk et al. 2005]. The high pavement design temperature is defined at a depth of about $20 \mathrm{~mm}$ below the pavement surface and the low pavement design temperature at the surface of the pavement with the following correlations proposed based on a pavement solar absorption of 0.9 , radiation transmission coefficient through air of 0.81 , an atmospheric radiation of 0.7 and an average wind speed of $4.5 \mathrm{~m} / \mathrm{s}$. The maximum and minimum surface temperature recommended by Superpave is accordingly given by the follow equations.

$$
\begin{aligned}
& \mathrm{T}_{\mathrm{s}(\max )}=\mathrm{T}_{\text {air(max) }}-0.00618 \mathrm{Lat}^{2}+0.2289 \mathrm{Lat}+24.4 \\
& \mathrm{~T}_{\mathrm{s}(\min )}=0.859 \mathrm{~T}_{\text {air(min) }}+1.70
\end{aligned}
$$

Where $\mathrm{T}_{\mathrm{s}(\min )}=$ the daily minimum surface temperature in ${ }^{\circ} \mathrm{C}$

$\mathrm{T}_{\mathrm{s}(\max )}=$ the daily maximum surface temperature in ${ }^{\circ} \mathrm{C}$

$\mathrm{T}_{\operatorname{air}(\max )}=$ the daily maximum air temperature in ${ }^{\circ} \mathrm{C}$

$\mathrm{T}_{\text {air(min) }}=$ the daily minimum air temperature in ${ }^{\circ} \mathrm{C}$

Lat=latitude of the location

The Superpave algorithm for maximum and minimum pavement temperatures at depth is shown as Equations 5.4 and 5.5, respectively.

$\mathrm{T}_{\mathrm{d}(\max )}=\left(\mathrm{T}_{\mathrm{s}(\max )}+17.8\right)\left(1-2.48 \times 10^{-3} \mathrm{~d}+1.085 \times 10^{-5} \mathrm{~d}^{2}-2.441 \times 10^{-8} \mathrm{~d}^{3}\right)-17.8$

$\mathrm{T}_{\mathrm{d}(\min )}=\mathrm{T}_{\mathrm{s}(\min )}+5.1 \times 10^{-2} \mathrm{~d}-6.3 \times 10^{-5} \mathrm{~d}^{2}$

Where $\mathrm{T}_{\mathrm{d}(\max )}=$ Maximum daily asphalt temperature at depth $\mathrm{d}$ in ${ }^{\circ} \mathrm{C}$

$\mathrm{T}_{\mathrm{d}(\min )}=$ Minimum daily asphalt surface temperature in ${ }^{\circ} \mathrm{C}$ from Equation 5.3

$\mathrm{d}=$ depth in $\mathrm{mm}$

How ever the Superpave equation is valid for the position of the sun in the summer sky. On the other hand the maximum surface temperature is expressed as follow [Denneman 2007]. The model includes the Zenith angle which allows for seasonal and daily variations in the solar energy potential.

$\mathrm{T}_{\mathrm{s}(\max )}=\mathrm{T}_{\operatorname{air}(\max )}+24.5\left(\cos \mathrm{Z}_{\mathrm{n}}\right)^{2} . \mathrm{C}$

$\mathrm{T}_{\mathrm{s}(\min )}=0.89 \mathrm{~T}_{\text {air(min) }}+5.2$

Where $\mathrm{T}_{\mathrm{s}(\max )}=$ the daily maximum asphalt surface temperature in ${ }^{\circ} \mathrm{C}$

$\mathrm{T}_{s(\min )}=$ the daily minimum asphalt surface temperature in ${ }^{\circ} \mathrm{C}$

$\mathrm{T}_{\text {air(max) }}=$ the daily maximum air temperature in ${ }^{\circ} \mathrm{C}$

$\mathrm{T}_{\text {air(min) }}=$ the daily minimum air temperature in ${ }^{\circ} \mathrm{C}$

$\mathrm{Z}_{\mathrm{n}}=$ Zenith angle at midday

$\mathrm{C}=$ Cloud cover index

With $\quad \mathrm{C}=1.1$ if $\mathrm{T}_{\text {air(max })}>30^{\circ} \mathrm{C}$

$\mathrm{C}=1.0$ if monthly mean air temperature $<\mathrm{T}_{\text {air(max })}<30^{\circ} \mathrm{C}$

$\mathrm{C}=0.25$ if $\mathrm{T}_{\text {air(max) }}<$ monthly mean air temperature

The zenith angle is a function of the solar declination as shown in Equation 5.8 below:

$\cos \left(\mathrm{Z}_{\mathrm{n}}\right)=\sin ($ latitude) $\cdot \sin ($ declination$)+\cos ($ latitude $) \cos ($ declination$)$

Declination $=-23.45^{\circ} \cdot \cos \left[\frac{360^{\circ}}{365} \cdot(N+10)\right]$

Where $\mathrm{N}=$ day of the year (with $1^{\text {st }}$ of January $=1$ ) 
Further the pavement temperature at depth and any time of day can be used to predict [Denneman 2007] using Equation 5.10 at daytime and Equation 5.11 at nighttime.

$$
T_{d(t)}=T_{d(\min )}+\left[T_{d(\max )}-T_{d(\min )}\right] \sin \left[\pi \frac{\left(t-t_{r}-\beta\right)}{D L+2(\alpha-\beta)}\right]
$$

Where DL $=$ Day length

$\mathrm{t}=$ hour $\mathrm{t}$

$\mathrm{d}=$ depth in $\mathrm{mm}$

$\mathrm{T}_{\mathrm{d}(\mathrm{t})}=$ asphalt temperature at depth $\mathrm{d}$ at hour $\mathrm{t}$

$\mathrm{T}_{\mathrm{d}(\max )}=$ Maximum temperature at depth from Equation 7

$\mathrm{T}_{\mathrm{d}(\min )}=$ Minimum temperature at depth from Equation 8

$\mathrm{t}_{\mathrm{r}}=$ time of sunrise

$\alpha=$ time lag between 12 noon and occurrence of maximum pavement temperature (refer Equation 5.13)

$\beta=$ time lag between sunrise and occurrence of minimum asphalt temperature, the best fit found for âis 1.5 hours

$$
\begin{aligned}
& T_{d(t)}=T_{d(m i n)}^{n}+\left[T_{d\left(t_{s}\right)}-T_{d(m i n)}^{n}\right]^{\left[\frac{\gamma\left(t-t_{s}\right)}{24-D L+\beta}\right]} \\
& \mathrm{t}_{\mathrm{s}}=\text { time of sunset } \\
& \mathrm{T}^{\mathrm{n}} \mathrm{d(min)}=\text { minimum temperature at depth } \mathrm{d} \text { on the next } \\
& \mathrm{T}_{\mathrm{d}(\mathrm{ts})}=\text { is temperature at sunset calculated using Equation } \\
& \gamma=\text { a decay parameter, assumed to be } 3.9
\end{aligned}
$$

$$
\begin{aligned}
& D L=\frac{2}{15} \times \cos ^{-1}[-\tan (\text { latitude }) \times \tan (\text { solardeclination })] \\
& \alpha=2+\frac{d}{50}
\end{aligned}
$$

Based on the above equation the temperature response of a pavement can be computed.

On the other hand [Wellner and Kayser 2008] the temperature distribution with in the asphalt layers has been investigated. In the project (conducted at Dresden university, Germany), temperature zones had been developed which included information about the frequencies of pavement surface temperature and their regional variation. The heat balance equation was adopted to compute the surface temperature with the aid of various climatic boundary conditions. The temperature gradients had been described by mathematical function (as given in Equation 5.14 through 5.17) and related to the pavement surface temperature. It has been suggested that accurate classification of the temperature gradient had been possible by using the intensity of daily temperature change and the time of the day. Computations were carried out using different pavement structures based on measured temperatures which had been recorded with in road condition and weather information system at different locations in the country. The temperature gradient within the pavement section is given as follow.

$\vartheta\left(\mathrm{x}, \vartheta_{\mathrm{FO}}\right)=\mathrm{A} \cdot \ln (0.01 \mathrm{x}+1)+\mathrm{B}$

Where $\vartheta\left(x, \vartheta_{\mathrm{FO}}\right)$ temperature at depth $\mathrm{x}$ for a given surface temp $\vartheta_{\mathrm{FO}}$ $\mathrm{x}$ - depth

A, B surface temperature dependent coefficients 


\begin{tabular}{|c|c|c|}
\hline $\boldsymbol{\vartheta}_{\mathrm{FO}}$ & A & B \\
\hline$<-5^{\circ} \mathrm{C}$ & 4,5 & $-7,5$ \\
\hline bis $0^{\circ} \mathrm{C}$ & 2,5 & $-2,5$ \\
\hline bis $5^{\circ} \mathrm{C}$ & 0,7 & 2,5 \\
\hline bis $10^{\circ} \mathrm{C}$ & 0,1 & 7,5 \\
\hline bis $15^{\circ} \mathrm{C}$ & 0,3 & 12,5 \\
\hline bis $15^{\circ} \mathrm{C}$ & 0,4 & 17,5 \\
\hline bis $25^{\circ} \mathrm{C}$ & $-1,6$ & 22,5 \\
\hline bis $30^{\circ} \mathrm{C}$ & $-4,0$ & 27,5 \\
\hline bis $35^{\circ} \mathrm{C}$ & $-6,2$ & 32,5 \\
\hline bis $40^{\circ} \mathrm{C}$ & $-8,5$ & 37,5 \\
\hline bis $45^{\circ} \mathrm{C}$ & $-10,5$ & 42,5 \\
\hline$>45^{\circ} \mathrm{C}$ & $-12,0$ & 47,5 \\
\hline
\end{tabular}

Table 5.1 Values of A and B for different surface temperature [Wellner and Kayser 2008]

The time dependent temperature of the pavement has been evaluated for a given average surface temperature and amplitude of the periodical temperature change of the surface using the following equation [Wellner and Kayser 2008].

$$
\vartheta(x, t)=\vartheta_{M}+\Delta A_{\vartheta} \cdot e^{-\zeta} \cdot \cos (\psi \cdot t-\zeta)
$$

With:

$$
\begin{aligned}
& \zeta=\mathrm{x} \cdot \sqrt{\frac{\pi}{\mathrm{a} \cdot \mathrm{t}_{\mathrm{p}}}} \\
& \psi=\frac{2 \cdot \pi}{\mathrm{t}_{\mathrm{p}}} \\
& \mathrm{a}=\frac{\lambda_{w}}{\rho \mathrm{c}_{\mathrm{p}}}
\end{aligned}
$$

$\vartheta(\mathrm{x}, \mathrm{t})$ - temperature of the material at depth $\mathrm{x}$ at time $\mathrm{t}\left({ }^{0} \mathrm{k}\right)$

$\vartheta_{\mathrm{M}}-$ average surface temperature $\left({ }^{\circ} \mathrm{k}\right)$

$\Delta \mathrm{A}_{\vartheta}$ - amplitude of the periodical temperature change at the surface

a - temperature conductivity

$\rho$ - density

$\lambda_{\mathrm{w}}-$ heat conductivity

$c_{p}$ - specific heat capacity

$\mathrm{t}_{\mathrm{p}}$ - period time

The above equation (5.15) implies that the change in surface temperature influence the temperature gradient of within the structure and the propagation depends on the conductivity of the material, the amplitude of periodical temperature change, cycle time and other thermal behavior of the system. 


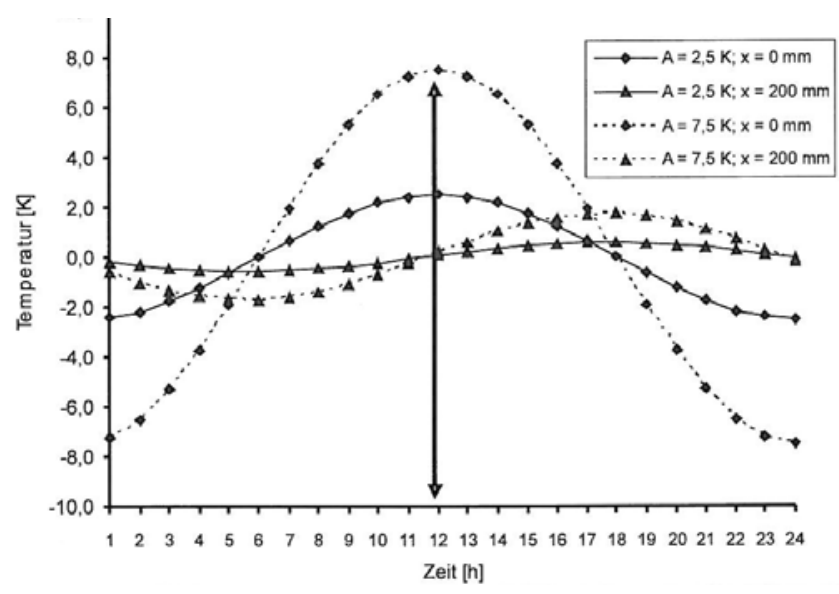

Figure 5.4 Temperature history at a given depths and amplitude [Wellner and Kayser 2008]

As a boundary condition in a periodic change, the surface temperature of the half space of the body is taken.

$\vartheta_{\mathrm{FO}}(\mathrm{t})=\vartheta_{\mathrm{M}}+\Delta \mathrm{A}_{\vartheta} \cdot \cos (\psi \cdot \mathrm{t})$

$\vartheta_{\mathrm{FO}}(\mathrm{t})$ - surface temperature of the material at time $\mathrm{t}\left({ }^{0} \mathrm{k}\right)$

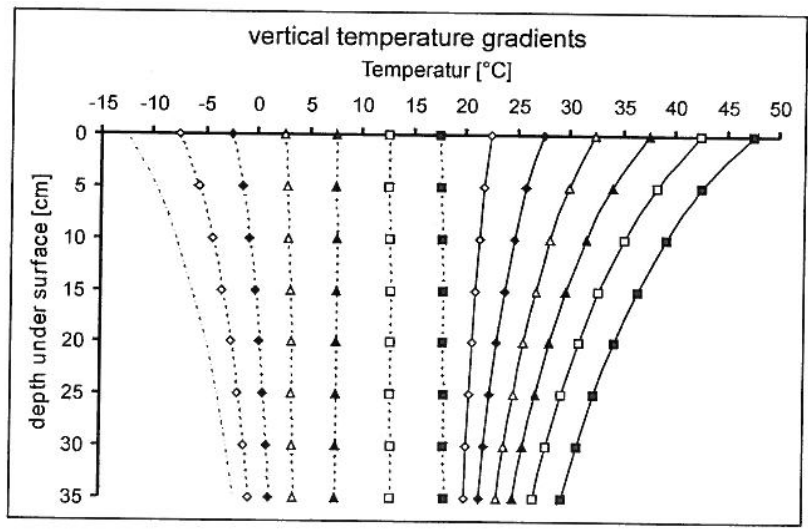

Figure 5.5 Temperature gradient for defined surface temperatures [Wellner and Kayser 2008]

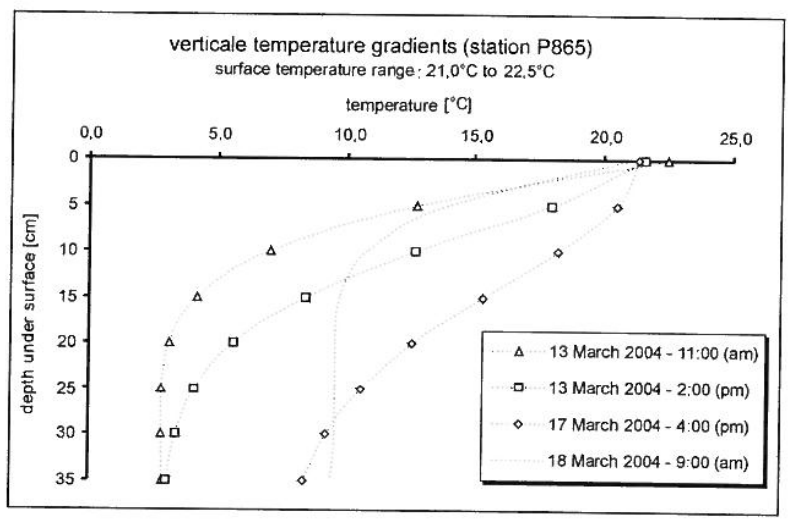

a. temperature range between $21-22.5^{\circ} \mathrm{C}$

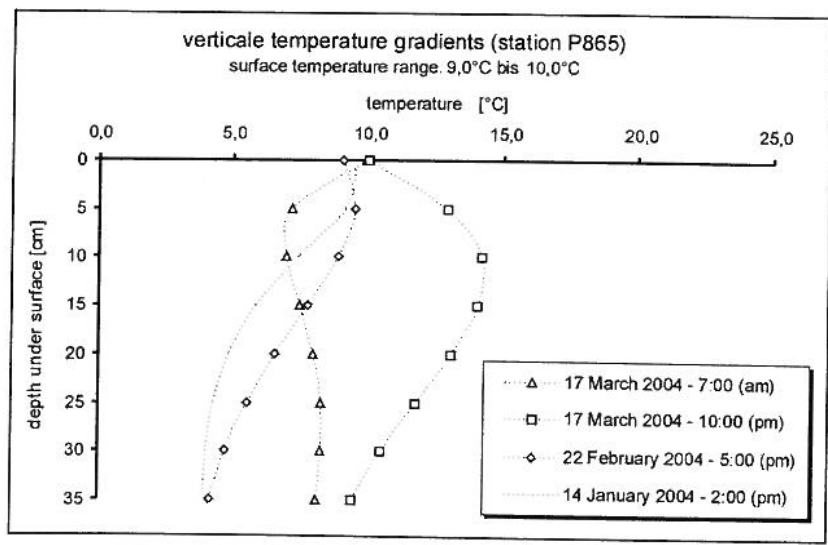

b. temperature range between $9-10{ }^{\circ} \mathrm{C}$

Figure 5.6 Vertical temperature gradient for various days and times for different temperature ranges (station P865Inntal Dreieck) [Wellner and Kayser 2008] 


\subsection{Thermal Properties of AC Materials}

Temperature is one of the most dominant factors that impact the variation in modulus of asphalt concrete layers. It has been indicated that the property of AC changes significantly with change in temperature and behaves as viscoelastic or plastic material at high temperature and elastic and brittle at low temperature. Techniques for thermal distress minimization rely on the selection of resistant materials to extend pavement life and to reduce the frequency and cost of maintenance and rehabilitation activities. Mix design and analyses system that specifically address resistance to thermal cracking provide a tool to select mixtures with sufficient resistance to stresses induced by specific environmental conditions and evaluate the mixtures in terms of predicted performance in a particular environment.

Limiting values for binder temperature susceptibility have traditionally been recommended to minimize both types of thermal cracking. Material selection based on binder properties alone may not be sufficient to predict improvements in mixture resistance to thermal distress. A reliability based mix design and analysis system that specifically addresses resistance to thermal distress provides a tool for the engineer to select mixtures with sufficient resistance to stresses induced by specific environmental conditions and evaluate selected mixtures in terms of predicted performance in a particular environment. This type of system can be developed in conjunction with a laboratory evaluation of asphalt-aggregate mixture resistance to both rutting and thermal cracking.

Thermal cracking of asphalt pavements is a very serious pavement distress, which has been linked to asphalt binder properties at low temperatures, asphalt mixture mechanical properties, thermal properties and pavement configuration (layer types and thicknesses). Several models [Buttlar et al. 2009, Chehab and Kim 2005, Epps 2000, Li and Marasteanu 2005] developed for thermal cracking are related to crack development at the surface of a pavement at low temperature. Epps [2000] analyzed and modeled the thermal effect in asphalt concrete for fatigue and permanent deformation. In his findings, he described that tensile stresses responsible for cracking were induced in the asphalt concrete surface layer of the pavement structure as temperature decreased and contraction was restrained by underlying layers. Both rapid shortterm aging during plant mixing and construction and slower long-term aging in the field stiffened the mixture and increased the stress induced on cooling. When the induced stress exceeded the tensile strength of the mixture (low temperature cracking) or when the effect of thermal cycling exceeded the mixture fatigue resistance (thermal fatigue), transverse cracks resulted from the maximum stress in the longitudinal direction of the pavement.

Low temperature cracking of asphalt mixtures has been studied and a fracture mechanics concept has used to investigate the fracture properties of the mixtures [ $\mathrm{Li}$ and Marasteanu 2005]. They described the fracture behavior of asphalt mixtures using cohesive zone model under monotonic loading. The simulation was calibrated with the experimental results from a newly proposed semi circular bend (SCB) test. In the numerical simulation, the asphalt mixture was assumed to behave elastically. Three simulations were performed for each asphalt mixture using material parameters measured at $-20,-30$ and $-40{ }^{\circ} \mathrm{C}$, respectively. The asphalt pavement was assumed to be the equivalent of many repetition of a standard unit with the length of the mean crack spacing. They reported that the asphalt mixture made with asphalt binder with lower PG limit performed better in terms of low temperature cracking and all three asphalt mixtures were more fracture resistant at higher temperature $\left(-20^{\circ} \mathrm{C}\right)$ than at lower temperature $\left(-40^{\circ} \mathrm{C}\right)$. A parametric study of the relative influence of material parameters on the low temperature cracking was performed using the $58-34$ mixture at $-30^{\circ} \mathrm{C}$ as the control case. They concluded that higher modulus and/or lower tensile strength require a smaller temperature drop to initiate the low temperature cracking and higher fracture energy does not increase significantly the temperature drop necessary for initiating the crack. However, it leads to a larger temperature drop to propagate the low temperature cracking to the bottom of the asphalt layer. On the other 
hand it has been concluded that field performance with hard asphalts indicated no susceptibility to low temperature or thermal fatigue cracking [Corté 2001].

The variation in modulus is typically related to the average temperature of a layer. However, the temperature gradient within a layer also plays a role in that relationship. To properly implement standard pavement design procedure or to develop realistic performance-based specifications, it is important to reliably predict and quantify the daily and seasonal variations of the moduli of different layers.

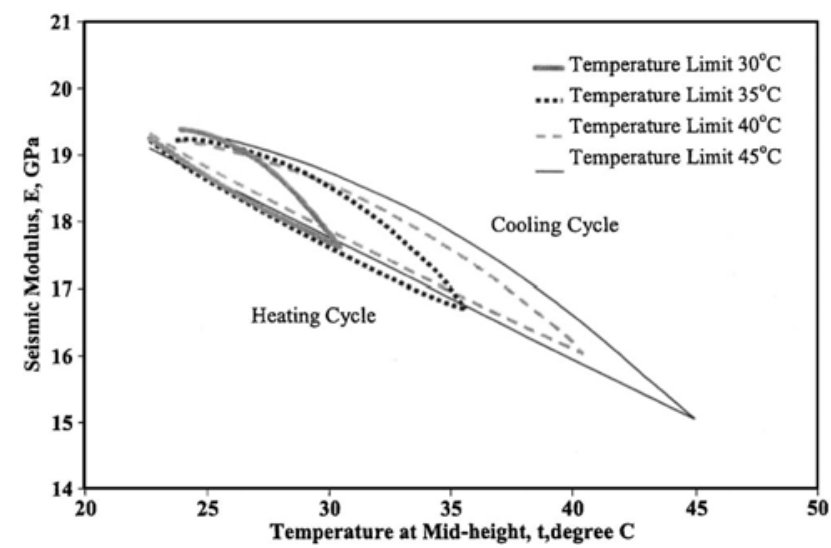

a. during various cooling and heating cycles

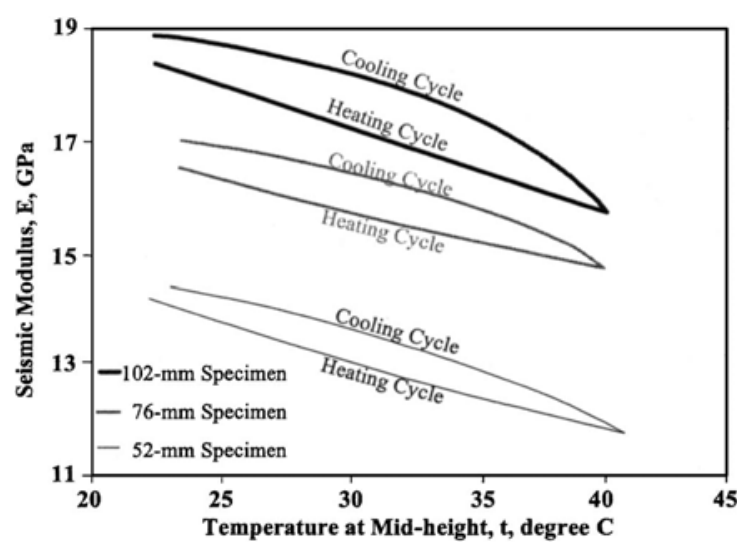

b. during single cooling and heating

Figure 5.7 Variation in modulus and impact of layer thickness during heating and cooling cycles [Nazarian and Alvarado 2006]

Characterization of the variation in modulus of the asphalt concrete with temperature is essential in any pavement design or analysis algorithm. The variations in temperature at the top and at the bottom of the specimen with the mid height temperature are [Nazarian and Alvarado 2006] different in cooling and heating. The rate of reduction in modulus with temperature is dependent on the temperature gradient of the specimen.

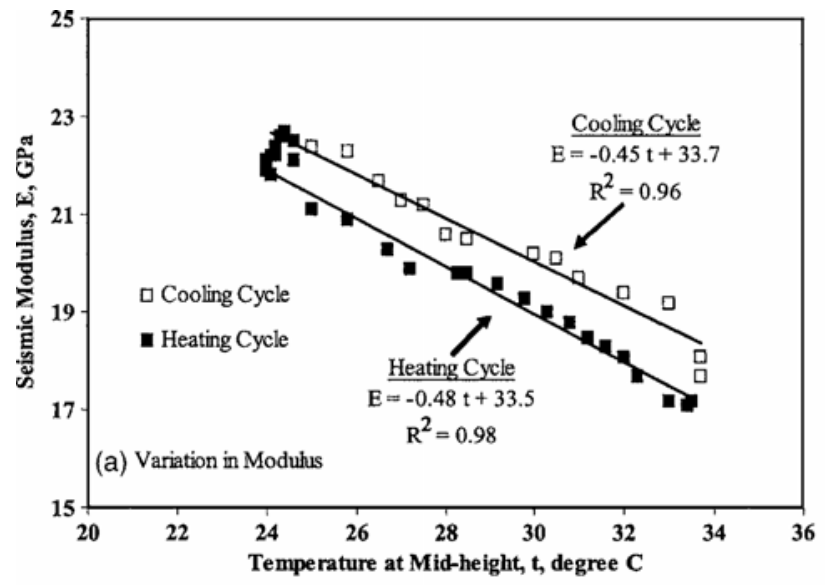

Figure 5.8 Impact of temperature gradient on modulus [Nazarian and Alvarado 2006]

It has been reported [Park et al. 2001] that surface deflection and backcalculated layer moduli of asphalt concrete pavements have been significantly affected by the temperature of the AC layer. 

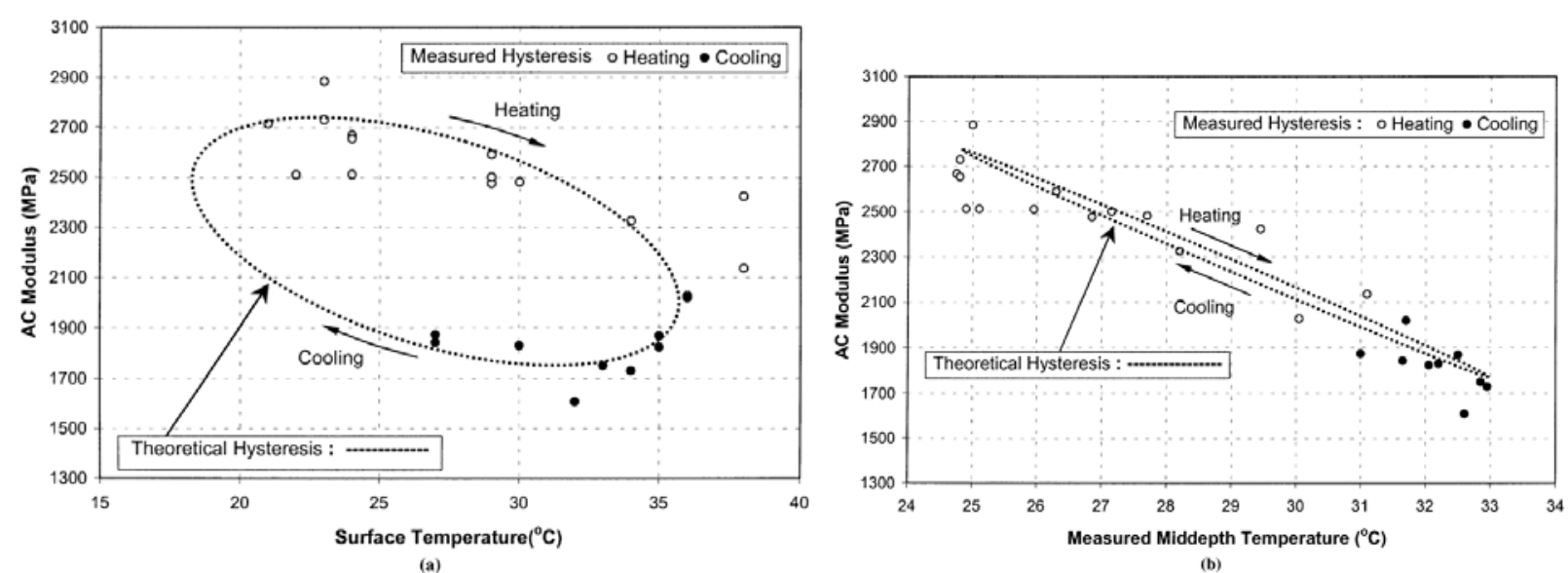

(a)

Figure 5.9 Measured temperatures at surface and mid-depth versus AC modulus [Park et al. 2001]

Rutting is a major distress form in asphalt pavements, especially when the ambient temperature is high as in a hot tropical climate region or during the summer months of temperate countries. Rutting in asphalt concrete mixes under traffic loading occurs predominantly at elevated temperatures. At elevated temperatures, AC mixes become softer and exhibit markedly different volume-change and shape-distortion deformation modes in terms of their sensitivity to temperature, rate of loading, and residual permanent deformation [Harvey et al. 2009].

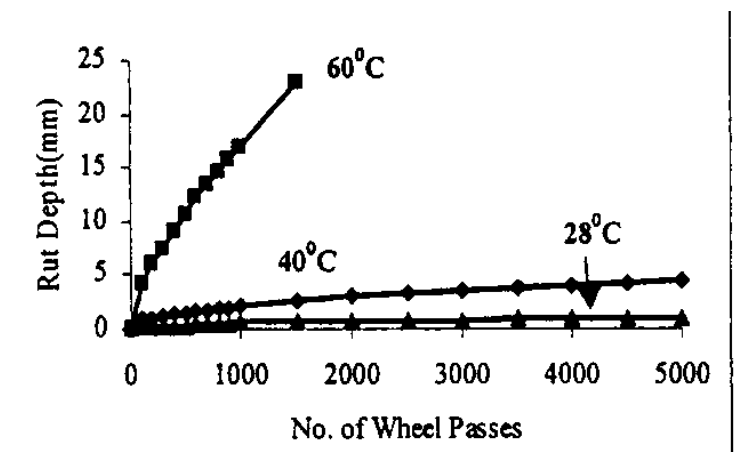

a. loading=690 $\mathrm{kpa}$, speed $=20$ passes $/ \mathrm{min}$

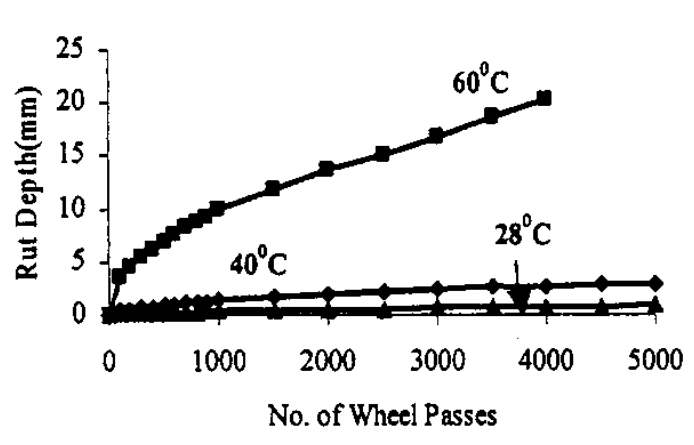

b. loading $=690 \mathrm{kpa}$, speed $=40$ passes $/ \mathrm{min}$

Figure 5.10 Rut depth variations with change in temperature and rate of loading [Fwa et al. 2004]

The hot-mix asphalt viscoelastic properties studies carried [Elseifi et al 2006] at intermediate and high temperatures indicated that at high temperatures or under slow moving loads, HMA exhibited a purely viscous flow, which resulted in load-associated distresses such as pavement rutting. However he concluded that, at low service temperatures or under rapidly applied loading, HMA became progressively harder and eventually brittle, which made it vulnerable to non-load-associated distresses such as low-temperature cracking. 


\section{Asphalt Concrete Modeling}

A great deal of work has been devoted in the past to the material characterization of rutting and fatigue crack prediction [Archilla and Madanat 2000 and 2001, Bahuguna1 et al. 2006, Benedetto et al. 2004, Capros et al. 2008, Fwa et al. 2004, Garba 2002, Ghuzlan and Carpenter 2003, Lundström et al. 2003]. It is believed that rut, fatigue damage, creep and degradation of the modulus of elasticity under cyclic loading, aging and softening at high temperature are some of the root causes of deterioration of asphalt concrete. Models developed so far predict such damages and their progress.

\subsection{Rut Modeling}

Permanent deformations have been the focus of much research in pavement engineering over the past 50 years [Schwartz and Kaloush 2009]. Most of the available permanent deformation models in the literature are empirical or semi-mechanistic with limited fundamental material characterization other than through elastic or quasi-elastic properties.

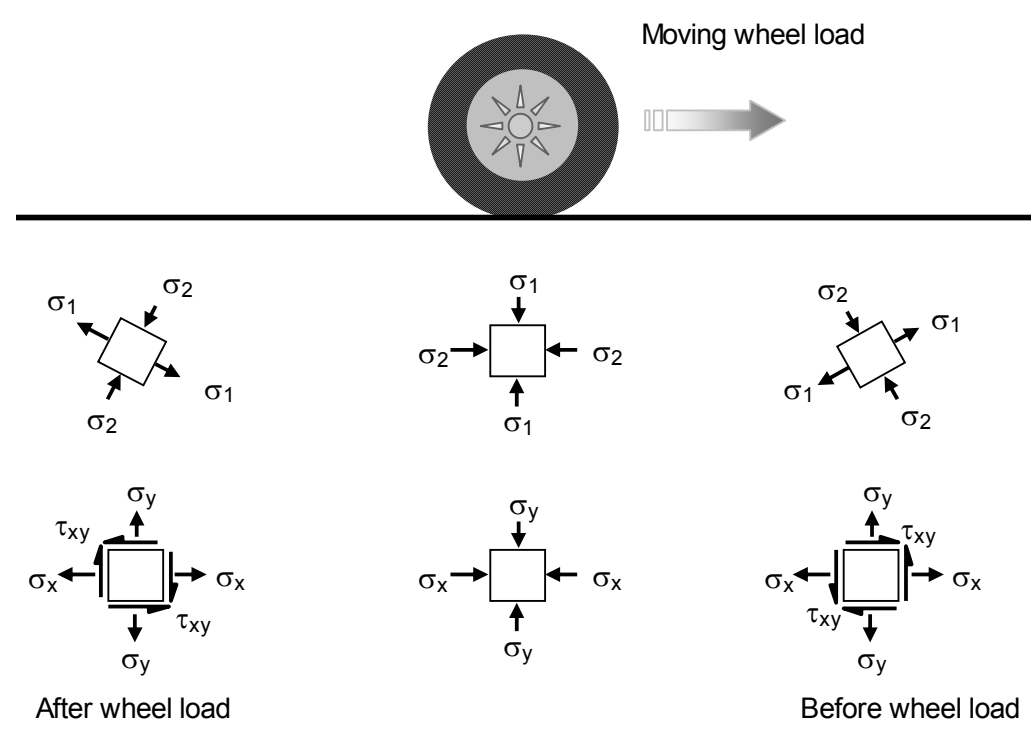

Figure 6.1 Orientation of principal stresses with respect to position of moving load

Determining the states of stress and strain in a pavement section under a load is an essential step in any rut-depth predictive methodology. Use of a suitable mathematical model of the pavement system and realistic material properties are closely interrelated. With increases in axle loads, load repetitions, tire pressures, and asphalt-concrete thicknesses, a need has raised for methodology to predict rut depths in advance of construction under the prevailing circumstances in order to mitigate potential safety problems.

Along with the development of analysis procedures which permit estimates of stresses, strains, and deformations resulting from traffic loads, pavement design systems have evolved which include provisions for rutting prediction. These have been referred to as analytically based, mechanistic, or mechanistic-empirical procedures.

A number of these procedures include criteria for limiting values of subgrade strain to levels that preclude rutting at the pavement surface. Some have recommended limitations on vertical subgrade stress (rather than strain). Others have utilized statistically based rut depth prediction equations. Design limitations on strain or stress are based on the assumption that, if the 
maximum vertical compressive strain or stress at the surface of the subgrade or at any specific point is less than a critical value, then rutting will be limited to a tolerable level for a specified number of load applications.

Unfortunately, such methodology does not necessarily preclude rutting which might occur in the asphalt-bound layer. There are many models for researching permanent deformation of asphalt mixture, including the empirical and mechanistic models, and each model has its own advantages and disadvantages.

The evaluation of asphalt concrete mixes for their tendency to cause rutting has been an active area of research for several years, which aim to mitigate or reduce rutting in flexible pavements. The prediction of rutting development and progress in asphalt pavement is an essential element for pavement design. Many rutting models are developed to date to estimate and/or characterize the extent and behavior of deformation and an attempt has been made to incorporate them in road design manuals. Most theoretical works in asphalt concrete in a period spanning the last several years have been toward modeling the elastic, viscoelastic/viscoplastic (rheological) behavior of the material [Bahuguna1 et al. 2006, Chehab and Kim 2005, Drescher et al. 1993, Elseifi et al. 2006, Shen and Kirkner 2001].

An empirical rutting progression model using experimental data was developed [Archilla and Madanat 2000], with a thawing index variable incorporated to capture the effects of the environmental factors. The thawing index was related to temperature fluctuation around freezing point. The effect of thawing would be the greatest when there has been considerable accumulated freeze from previous periods and the temperatures in the current period are substantially above zero. In such cases there would be large amounts of water in the pavement structure with consequent detrimental effects. Even though it was an improvement over the traditionally empirical model, the author admitted that it had its own limitation as it didn't take in to account the behavior of asphalt concrete mixes and the rutting contribution due to asphalt concrete rutting.

A mechanistic-empirical rutting model was developed [Chen et al. 2004] to account for material property changes in the progression of rutting over time and the cumulated compressive plastic strains of all layers on the basis of traffic loading. They established a three-dimensional dynamic finite-element simulation to calculate pavement responses that were input into the rutting model for predicting permanent deformation. In their simulation the traffic flow was separated into four levels of service dependent on the travel speed $(92,60,45,20 \mathrm{kph})$ and the duration time calculated by contacted length divided traffic speed. Asphalt mixtures were modeled as timeand temperature-dependent viscoelastic materials. The time-dependent properties were represented by instantaneous and long-term shear moduli. The temperature effect was considered through the changes in shear modulus values at different temperatures. They observed that a significant increase in strain magnitude as the vehicle speed decreases. The maximum vertical compressive strain at the top of the surface layer corresponding to a travel speed of $20 \mathrm{kph}$ was two times greater than that of $90 \mathrm{kph}$. They concluded that traffic speed had showed significant impact on vertical strains at the top of the surface layer.

A mechanistic-empirical framework was presented [Uzan 2004] for evaluating permanent deformation in flexible pavements. His procedure used the "actual" temperature distribution in the asphalt layer at every hour in the whole design period. The stress distribution in the pavement sublayers was computed using a simplified scheme based on multilayer linear elastic systems. He concluded that the number of sublayers in the subgrade needs to be adjusted, depending on the stress level and permanent deformation potential of the material. In the case of the clayey subgrade used in this analysis and well-designed pavements, the contribution of the subgrade to permanent deformation was small.

A creep model was by Hua et al [Hua and White 2002] used to represent the viscoplastic strain as sole contributor to permanent deformation and characterize the time-dependent material properties of HMA in finite element analysis. Factors addressed in the models mainly included 
pavement geometry, boundary conditions, materials and loads. They concluded that tire print shape and tread pattern influences rutting shape and depth.

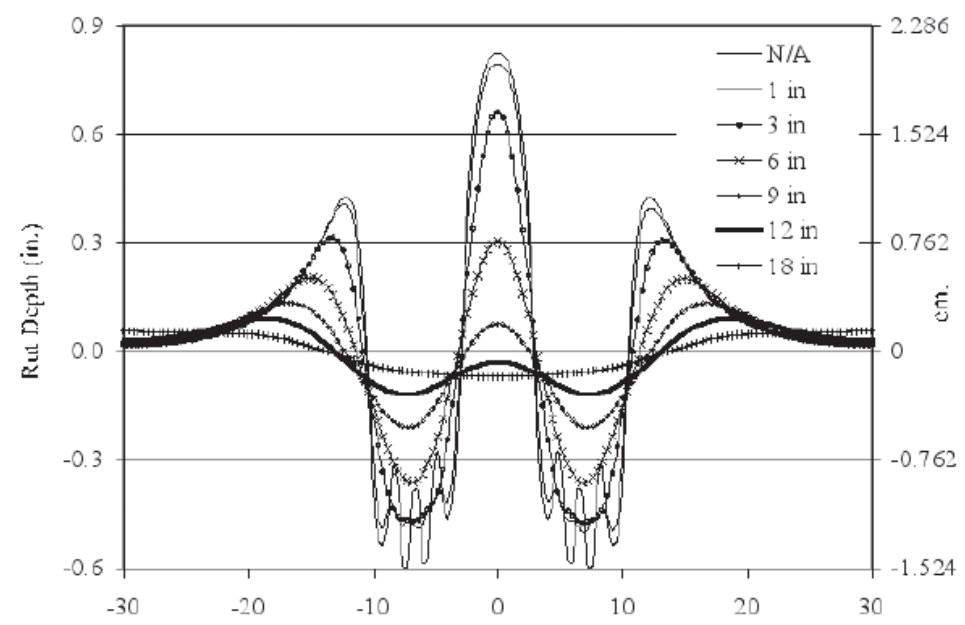

Figure 6.2 Rut accumulation at different with wander differences [Hua and White 2002]

A comprehensive matrix of pavement structures was analyzed using finite element analysis [White et al 2002] to simulate rutting failures in HMA surface mixtures, base courses, and subgrade. Results from this analysis clearly showed differences in transverse surface profiles and distortion parameters for different pavement failure modes. The analysis has provided strong evidence that a relationship exists between transverse surface profile and location of rutting in the pavement layers. They found good agreement between the predicted and field-observed location of failure in the pavement structure.

A micromechanical model for the steady-state deformation of idealized asphalt mixes presented [Deshpande and Cebon 2004] indicated that the deformation rate varied nonlinearly with the applied load and the maximum deformation rate was seen to occur below the road surface and at a radius equal to about half the contact width of the loading strip.

The constitutive model developed [Bahuguna et al 2006] has incorporated an insight about the

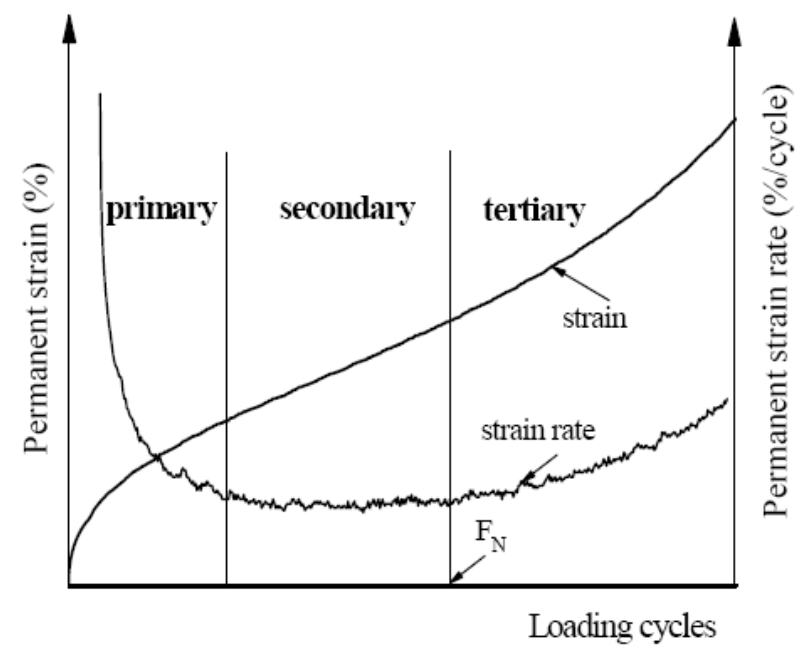

Figure 6.3 Permanent strain and strain rate versus the number of loading cycles

pattern of evolution of permanent deformation with an increasing number of cycles in the framework of the theory of generalized plasticity. The model was a representation of material 
behavior under multiaxial stress and strain states which was based on the theory of irreversible thermodynamics with internal variables. The constitutive model consisted of an elastic component in series with a plastic component and it captured the development of compressive stresses in the mix under shear loading. The plastic component of the model, which was based on the theory of generalized plasticity, described the irreversible part of the deformation and in particular the ratcheting (accumulation of permanent deformation with an increasing number of loading cycles) behavior exhibited by the mix. They concluded that models that were based on generalized plasticity had a significant computational advantage over models of classical plasticity.

It has been recommended the use of energy dissipation approach to assess the resistance to permanent deformation of bituminous mixtures [Widyatmoko et al 1999]. According to their remarked, the dissipated (loss) energy is the amount of energy which is dissipated by viscous flow and/or plastic flow, and leads to potential damage (e.g. permanent deformation) when a bituminous material is subjected to repetitive loading. They found the method was beneficial for accurately determining the end of the linear region from the curves of permanent strain versus number of loading cycles. An analysis procedure based upon a combined technique of strain rate and energy dissipation method was presented in their paper. They emphasized that during the deformation of a viscoelastic material, part of the total energy required to perform the work was dissipated through viscous losses, with the remainder of the deformational energy was being stored elastically. They concluded that the dissipated energy approach provided comprehensive information on the deformation mechanisms bituminous mixtures, which could be adopted for assessing the deformation resistance of bituminous mixtures.

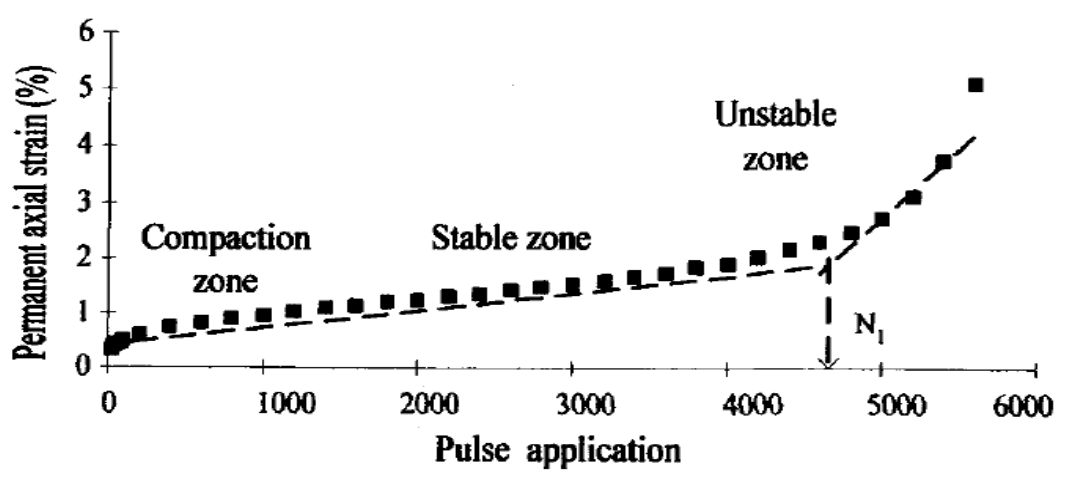

Figure 6.4 Plot of permanent strain curve as produced by the dynamic creep test [Widyatmoko et al. 1999]

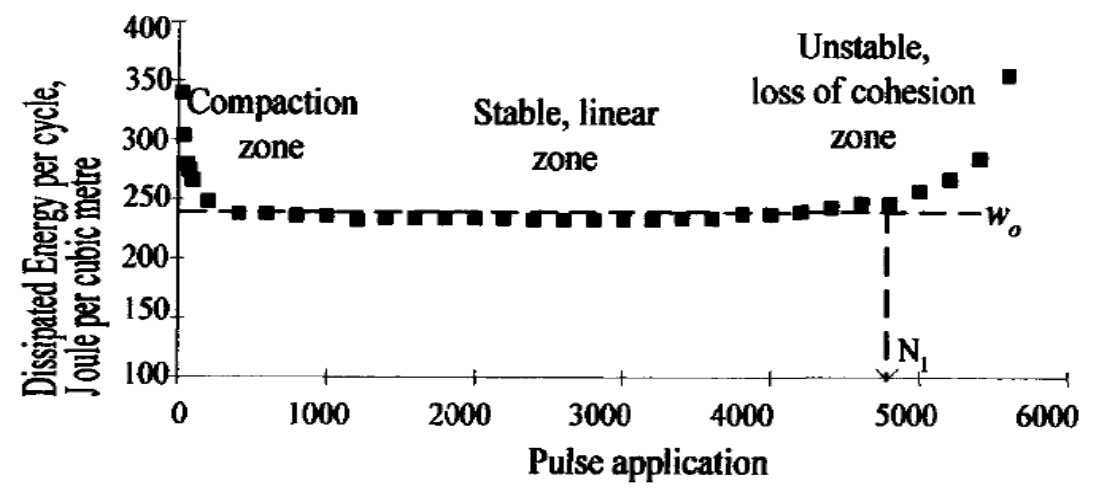

Figure 6.5 Plot of dissipated energy per cycle $\left[\mathrm{J} / \mathrm{m}^{3}\right]$ against the number of load repetitions [Widyatmoko et al. 1999]

An algorithm for determining the rheological parameters of asphalt mixes in the creep test using the Nottingham Asphalt Tester was presented [Szydlo and Mackiewicz 2005]. The rheological 
parameters were determined by approximating the creep curve with theoretical curves determined for the viscoelastic Burgers model.

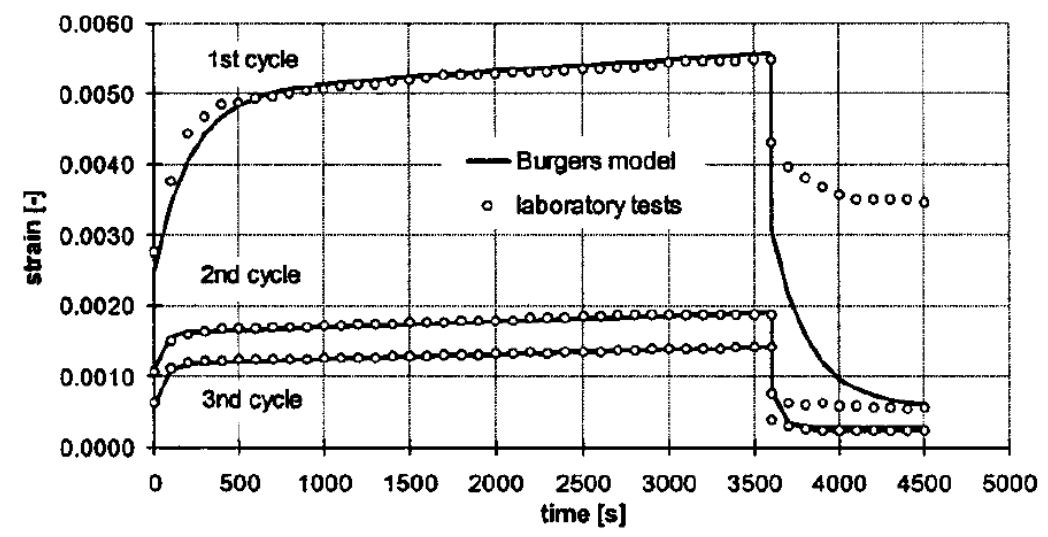

Figure 6.6 Creep curve approximation by burger model [Szydlo and Mackiewicz 2005]

The rut depth was calculated as the sum of the accumulated deformations after each loadingunloading cycle. The rut depth's sensitivity to a change in the rheological parameters was assessed. They stressed that the parameter $E_{1}$ (modulus of immediate elasticity) has no significant effect on rut depth. A pavement structure model's deformation sensitivity to changes in its rheological parameters was also analyzed. The rheological parameters of the considered asphalt mixes for the wearing course (mw1), the binder course (mb1), and the base course $(\mathrm{mbc} 1)$ were used to describe the rheological properties of the pavement courses.

\begin{tabular}{lccccc}
\hline $\begin{array}{l}\text { Course } \\
\text { (mixture) }\end{array}$ & $\begin{array}{c}\text { Temperature } \\
\left({ }^{\circ} \mathrm{C}\right)\end{array}$ & $\begin{array}{c}E_{1} \\
(\mathrm{GPa})\end{array}$ & $\begin{array}{c}\eta_{1} \\
(\mathrm{GPas})\end{array}$ & $\begin{array}{c}E_{2} \\
(\mathrm{GPa})\end{array}$ & $\begin{array}{c}\eta_{2} \\
(\mathrm{GPas})\end{array}$ \\
\hline $\begin{array}{l}\text { Wearing course } \\
(\mathrm{mw} 1)\end{array}$ & 42 & 0.192 & 2,490 & 0.197 & 7.74 \\
$\begin{array}{l}\text { Binder course } \\
(\mathrm{mb} 1)\end{array}$ & 35 & 0.223 & 2,350 & 0.225 & 9.51 \\
$\begin{array}{l}\text { Base course } \\
(\mathrm{mbc} 1)\end{array}$ & 25 & 0.527 & 2,490 & 0.965 & 13.3 \\
\hline
\end{tabular}

Table 6.1 Rheological parameters for pavement courses (roadway surface temp. $45^{\circ} \mathrm{C}$ ) [Szydlo and Mackiewicz 2005]

Figure 6.7 shows that parameter $E_{2}$ affects most strongly the deformation of the wearing course. Also, the effect of coefficient of viscosity $\eta_{1}$ for this course is less pronounced
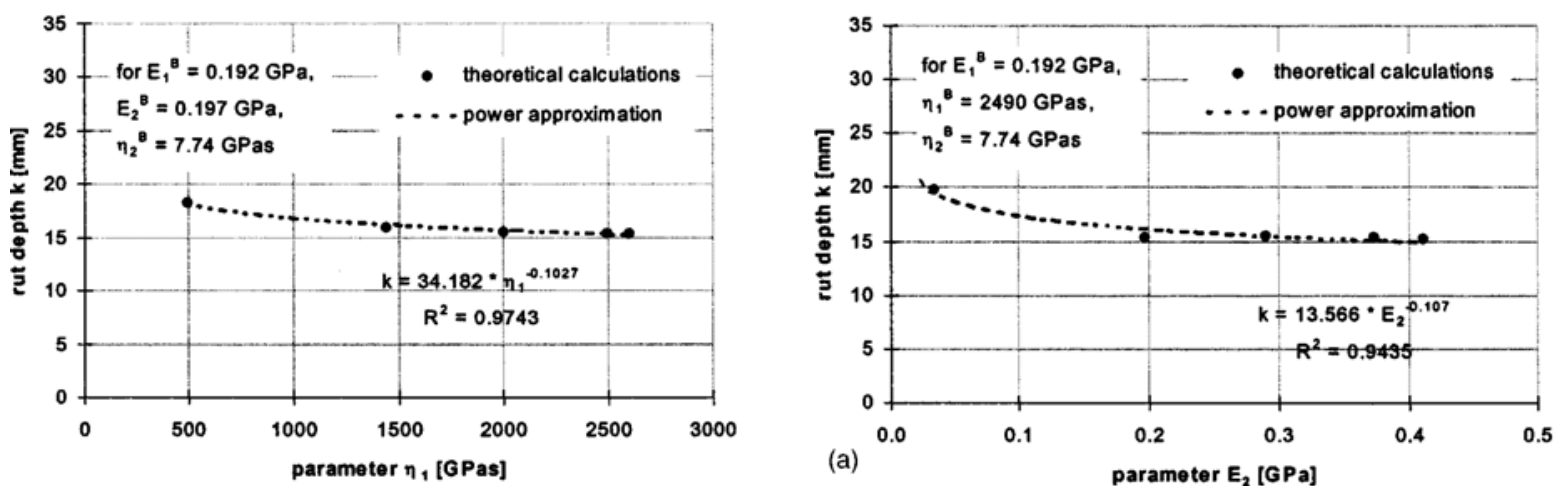

Figure 6.7 Relationships between wearing course rheological parameters and calculated rut depth [Szydlo and Mackiewicz 2005] 
According to Figure 6.8, the binder course exhibits the highest deformation sensitivity to changes in the rheological parameters, particularly to viscosity coefficient $\eta_{1}$. For this course the effect of a change in parameter $E_{2}$ is less pronounced.
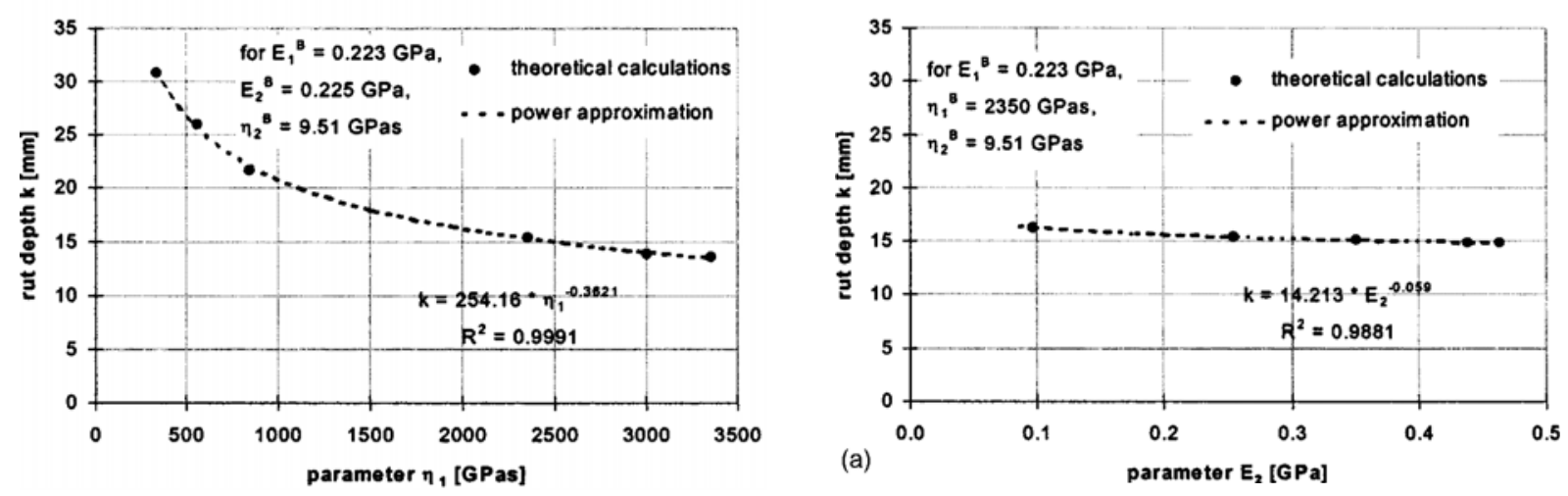

Figure 6.8 Relationships between binder course rheological parameters and calculated rut depth [Szydlo and Mackiewicz 2005]

It had been shown that parameter $\eta_{1}$ has similar significant effect on rutting for the base course. It was concluded that the viscosity coefficient $\eta_{1}$ and the delayed elasticity coefficient $E_{2}$ have a significant effect on rut depth in the rutting. According to their analysis, the deformation sensitivity of the viscoelastic courses in a pavement structure model showed that the binder course's rheological parameters, especially the viscosity coefficient $\eta_{1}$, contribute most to rutting in the pavement.

Stiff mixes was used [Walubita et al. 2008] with high modulus values (on the order of about 800 to about $2400 \mathrm{ksi}$ ) and they concluded that the measured in-situ field surface deflections have also generally remained very low even under summer temperatures; an indication that the mixes are also less temperature sensitive compared to the traditional Texas dense-graded mixes.

Simulation analysis on rutting of typical asphalt pavements is conducted [Huang et al. 2008] based on the actual climate situation, to study and analyze the behavior of rutting under different conditions of temperature fields and loading. They concluded that rutting usually occurs at the average daily air temperature of $20{ }^{\circ} \mathrm{C}$ or higher and the higher the average daily air temperature, the longer duration with the potential of rutting occurrence.

\subsection{Fatigue Modeling}

For the analysis of fatigue damage, the most commonly used primary responses are the horizontal tensile stress or strain at the bottom of the asphalt layer on the axis of the load, since analytical models generally predict that maximum tensile stresses/strains at that location. Pavement designers consequently infer the upwards propagation of fatigue cracks from the layer interface.

A great deal of work has been devoted in the past to the material characterization of fatigue crack propagation [Lee et al. 2000, Matthews et al. 1993, Lundström et al. 2003, Shen and Carpenter 2005]. One of the leading branches, fracture mechanics, bases the analysis on existing cracks. Another of the successful approaches was Weibull's theory which predicted, in a statistical manner, the distributed pattern of fatigue life of materials. The theory of micro crack development provides a better understanding of the mechanisms of fatigue failure by means of damage variables which represent the deterioration of a material element. A classical fatigue 
damage model known as nonlinear continuous damage (NLCD) advocated based on the concept of damage mechanics by constructing the damage evolution function according to the character of fatigue damage curves [Xiao et al. 1998]. In order to incorporate the high fatigue damage problem into the framework of continuum damage mechanics, a model based on a concept of microplastic strain can be developed from ductile damage model. The above mentioned are some main classical fatigue damage models in practice. A considerable amount of work also exists on fatigue cracking analysis and modeling. Fatigue analysis of the bituminous mix requires accurate assessment of the energy dissipated in the material during each loadingunloading cycle. The viscoelastic models developed for asphalt concrete have an important application in this domain as well.

There are several phenomenological fatigue models developed to predict fatigue cracking. These models are divided into two main types, the strain-based models which use a relation between the radial strain at the bottom of the asphalt concrete layer and the number of load applications to crack appearance in the pavement [Matthews et al. 1993]. Fatigue failure has traditionally been defined as the number of load cycles at which the initial stiffness or modulus is reduced by $50 \%$, for both strain and stress controlled test modes [Ghuzlan and Carpenter 2003, Kim et al. 2003]. Other criteria are based on changes in dissipated energy including dissipated energy ratio or damage accumulation ratio. It was also proposed by some researchers [Kim et al. 2003] that evaluation of changes in the phase angle during fatigue testing, a point showing the maximum phase angle was taken as a reasonable fatigue failure point, since the phase angle versus time curve shows a rapid loss of phase angle when asphalt mixtures stop accumulating distress.

Since this work focuses on the rutting, fatigue modeling is not discussed here in detail.

\subsection{AC Finite Element Modeling}

The finite element method (FEM) is a numerical analysis technique to obtain approximate solutions for a wide variety of engineering problems which are described either by partial differential equations or can be formulated as functional minimization. In finite element modeling, the region of interest is divided into small discrete shapes of finite dimensions, nodes are assigned to each element and then a function is chosen to interpolate the variation of the variable over the discrete element. Using the established finite element model (the elements and their interpolation functions) matrix equations are determined that express the properties of the individual elements. By combining the element matrix equations, the matrix of equations expressing the behavior of the entire system is formed. Then imposing values for certain variables at key boundary positions result in a set of simultaneous equations that can be solved for unknown displacements. From these displacements element strains and stresses can be calculated.

The critical step of any finite element (FE) simulation is to discretize the actual geometry of the structure using a collection of finite elements. Each finite element represents a discrete portion of the analyzed structure, which in turn, represented by many interconnected elements. The finite elements are joined by shared nodes which form the mesh. The element type, shape, and location, as well as the overall number of elements used in the mesh, affect the results obtained from a simulation [3].

The finite element method constitutes a particularly convenient tool for investigation of the influence of the material properties (e.g. strength, stiffness etc.) on the overall response of a structure. As stresses and strains are used to predict pavement distresses and thus the relative condition of the various layers in the pavement structure, the need for finite element analysis is significantly important. 


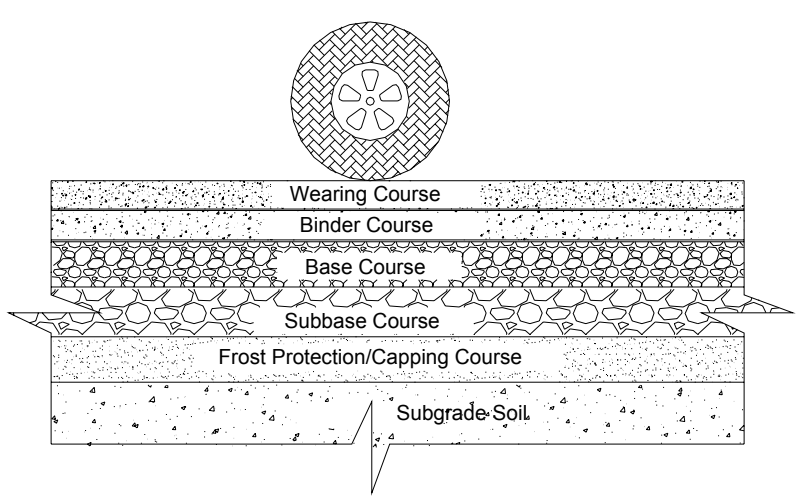

a. Typical pavement structure

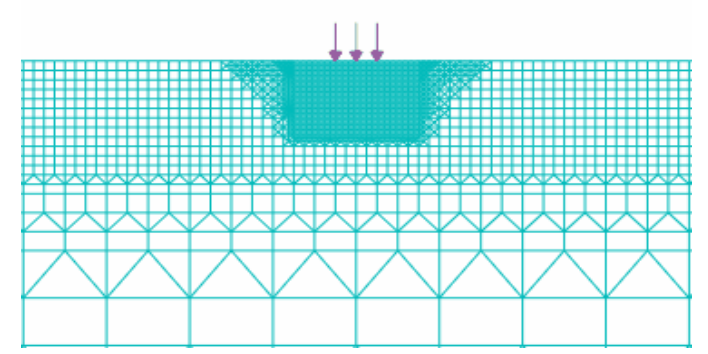

b. FE representation

Figure 6.9 Finite element modeling of asphalt concrete pavement

The FE method is by far the most universally applied numerical technique for flexible pavements [Yin et al. 2008]. Finite element modeling, if properly managed, can be very helpful as it can be used to directly evaluate the primary pavement structural responses or beyond that without resorting to potentially costly field experiments. It provides a modeling alternative that is well suited for applications involving pavement systems with inelastic materials, unusual boundary constraints or complex loading conditions. In the FEM analysis of a flexible pavement, the region of interest is divided into a number of small discrete volumes with the wheel loads over a certain contact area in order to obtain an approximate numerical solution for each individual volume rather than an exact closed-form solution for the whole pavement volume. The finite element can be further extended horizontally and vertically from the wheel to include all areas of interest within the influence of the wheel.
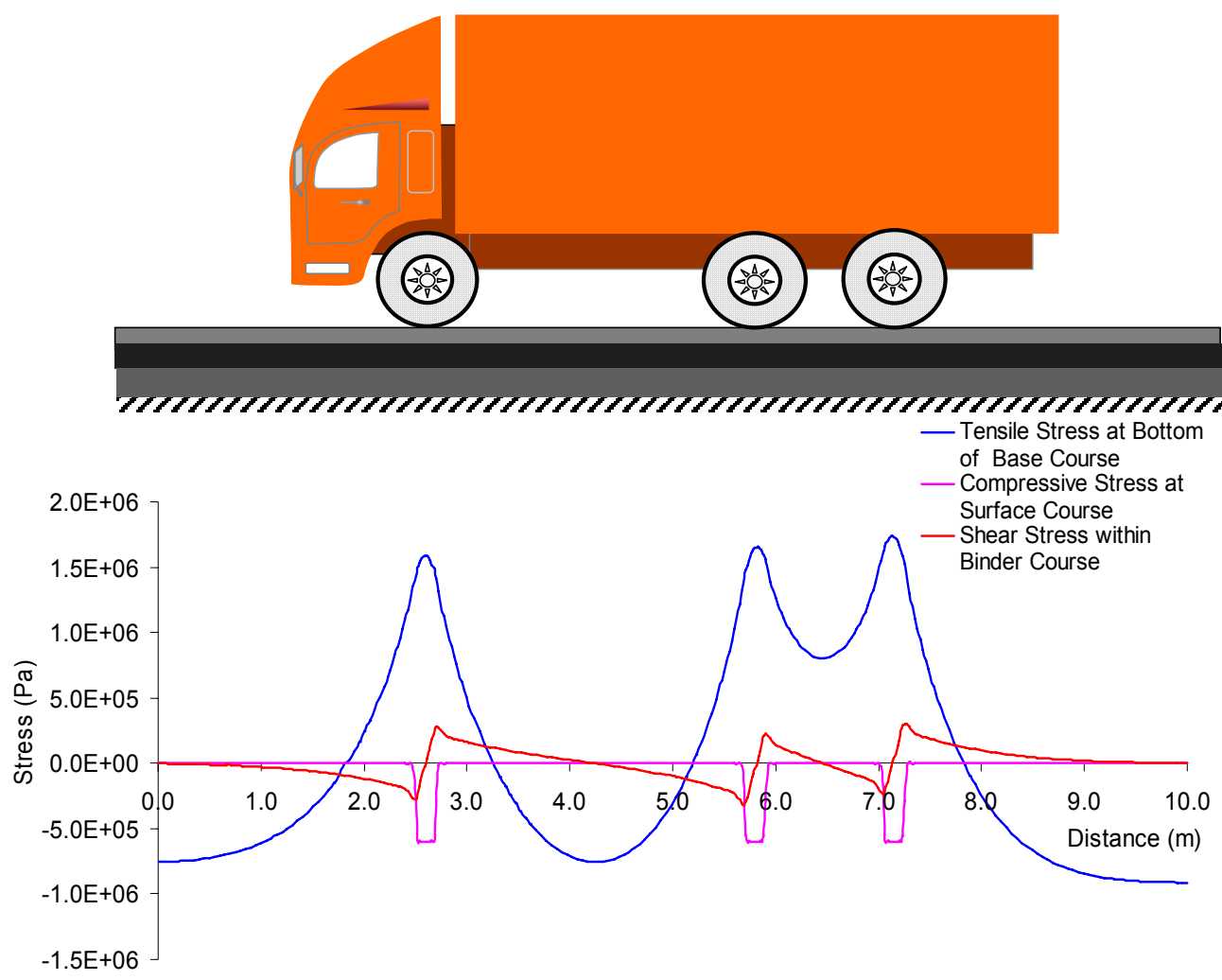

Figure 6.10 Critical stress states within the pavement structure with FE analysis 
Currently available finite element programs have the ability to consider linear and nonlinear elastic, viscoelastic and viscoplastic behavior of materials and to analyze asphalt concrete permanent deformation, fracturing, thermal cracking, reflection cracking, hardening and healing properties. Some studies carried out on FE analysis of AC pavements are highlighted below

The time effect on pavement response has been studied with the pavement temperature measured from instrumented thermocouples [Yin et al. 2008]. The time and temperature dependency of AC materials was integrated into one single factor, termed "effective temperature." Via this effective temperature, pavement responses under a transient load were predicted through finite element analysis. Viscoelastic behavior of AC materials was characterized through relaxation moduli, while the layers with unbound granular material were assumed to be in an elastic mode. Finite element analysis results revealed that the loading time had a more pronounced impact on pavement response in the summer. They emphasized that in the summer, the loading time had a considerable impact on both tensile and compressive strains. Consequently, a more pronounced time lag between the applied stress and the resulting strain was observed. In the spring, strains responses predicted from the effective temperature and measured temperature were almost identical. In other words, the pavement response during a cold season was barely influenced by the loading time. They suggested a possible reason that the AC was stiffer in spring compared to summer, and therefore the impact of the loading time was less pronounced.

The characteristics of hot-mix asphalt was studied [Elseifi et al. 2006] at intermediate and high temperatures and analyzed laboratory-based parameters by a three-dimensional finite element (FE) model to simulate pavement responses to vehicular loading at different temperatures and speeds. The FE model simulated the retardation of the response in the transverse direction and rapid relaxation of HMA in the longitudinal direction.

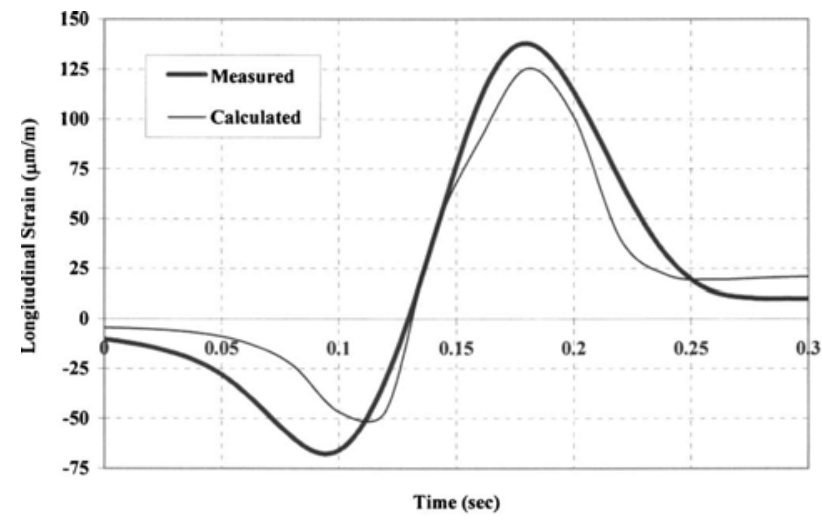

(a)

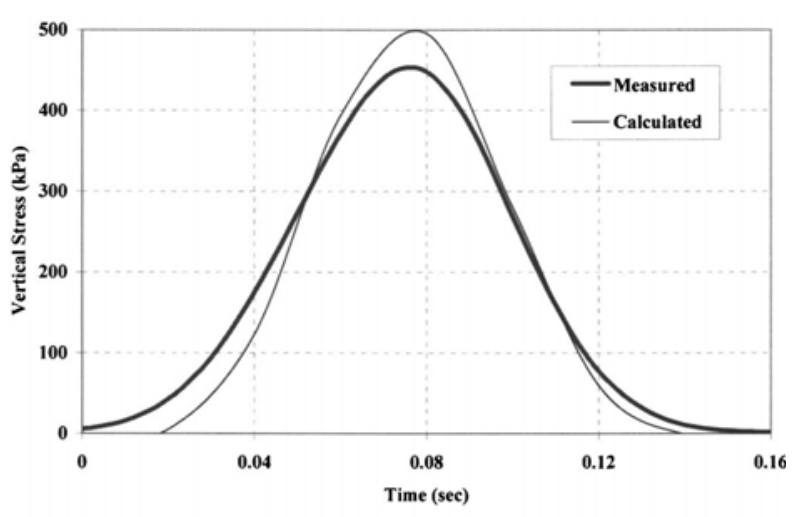

(b)

Figure 6.11 Comparison between measured and calculated (a) Longitudinal strain at the bottom of base course (viscoelastic); and (b) Vertical stress at the bottom of the wearing surface [Elseifi et al. 2006]

It has been [Elseifi et al. 2006] indicated that the viscoelastic FE model had predicted the vertical stress and the longitudinal strain at an error of less than 5\%. They concluded that as the speed had increased, the calculated strain significantly had also decreased.

The rutting of urban pavements [Ali et al 2009] was analyzed using finite-element modeling, which took into account the nonlinear behavior of the pavement materials with the complex traffic condition. A viscoplastic constitutive relation was used for the description of the behavior of the asphalt concrete layer, while an elastoplastic constitutive relation, based on the MohrCoulomb criterion, was used for the other layers. They remarked that rutting was largely affected by both the transverse wheel wander and the traffic circulation speed. Rutting decreased with the increased in traffic speed. They noted that assuming $20 \mathrm{~cm}$ of transverse wheel wander led to 
a reduction of about $20 \%$ in rutting due to the role of transverse wheel wander on the distribution of the load over a larger area of the pavement.

A series of finite element simulations was carried out [Saad et al 2006] to evaluate the benefits of integrating a high modulus geosynthetic into the pavement foundation by placing the reinforcement at different locations. They studied the effect on the fatigue and rutting deformations as well as analyzed how such effects were influenced by the base quality, thickness and the subgrade quality.

Development of finite element code specifically for asphalt concrete has been under progress. Mun [2003] studied the failure mechanisms of fatigue cracking in asphalt pavements using the finite element program that employed the viscoelastic continuum damage model for asphalt layer and a nonlinear elastic model for unbound layers. He developed finite element code, called VECD-FEP + + with the viscoelastic continuum damage model as the constitutive model of asphalt concrete and the universal model (resilient modulus model) for unbound materials. He concluded that the finite element analysis of various pavement load combinations showed significantly different failure mechanisms. According to his investigation the AC layer thickness had significant effects on the magnitude and location of damage. As the AC layer became thicker, the failure mechanism shifted from the bottom-up cracking with most damage at the bottom of the AC layer to the bi-modal mechanism where both bottom-up and top-down cracking occurred, or to the dominant top-down cracking in the case of the thicker AC layer. He further concluded that the non-uniform pressure caused larger stresses and greater damage than the uniform pressure did. The contribution of top-down cracking to the overall cracking in the AC layer became greater under the non-uniform pressure than under the uniform pressure.

It has been [Collop et al 2003] described the development and Finite Element implementation of a stress dependent elasto-visco-plastic constitutive model with damage based on the generalized Burger's model. Their model comprised linear elastic, linear viscoelastic and non-linear viscoplastic components. They explained that the permanent vertical strains in the non-stress dependent case were significantly lower than the permanent vertical strains in the stress dependent case and in the stress dependent case, there was a more localized area of high permanent compressive vertical strain directly under the load at approximately half depth in the asphalt compared to the non-stress dependent case where the distribution was more even.

A constitutive model was also developed [Erkens et al 2002] and implemented in the finite element system to simulate the initiation and propagation of damage in flexible pavement structures due to repeated loading. The simulations illustrated the influence of geometry and material characteristics on the development of damage.

Heterogeneous asphalt mixtures comprise of graded aggregates bound with mastic (asphalt binder plus fine aggregates and fines). The macro properties depend on the aggregate and mastic microstructure. A dramatic shift has been observed by many researchers towards a micromechanical finite element framework prediction of the damage properties of the asphalt mixtures [Dessouky et al. 2006, Masad and Somadevan 2002, Mun 2003]. The microstructure of an asphalt mixture is obtained from the scanned or digital images. The FE mesh is then generated within each aggregate and mastic sub domain. The aggregate and mastic elements share nodes on the aggregate boundaries for deformation connectivity. The viscoelastic mastic with specified properties can be incorporated with elastic aggregates in order to predict the viscoelastic properties of asphalt mixtures.

A microstructure-based viscoplastic continuum model was developed [Abbas 2004, Deshpande and Cebon 2004, Tashman et al. 2005, Tashman 2003] to simulate the permanent deformation of AC at high temperatures. The model linked important microstructure properties to the continuum behavior of AC in order to better predict permanent deformation. The model developed by Tashman et al. [2005] accounted for strain rate and confining pressure dependency, dilation, aggregate friction, anisotropy and damage. Furthermore, damage was 
included in the model based on the effective stress theory to account for crack and air void growth that significantly reduced the load-carrying capacity of the material.

A microstructure based finite element model of HMA was also developed [Masad and Somadevan 2002] under dynamic loading to evaluate the influence of the strain distribution on the HMA nonlinear response. Images of the microstructure were used to represent the geometry in a finite-element mesh which was captured on HMA sections using a digital camera that captured the details of the microstructure geometry. It has been concluded that the FE results of the HMA complex shear modulus as a function of temperature followed the same pattern as the experimental measurements. However, the calibration of the finite-element model showed that the shear modulus of the mastic needed to be about ten times and three times those of the binder in the fine and coarse mixes, respectively, in order for the model to match the measured HMA complex shear moduli. The finite-element model showed that the average mastic strain was about 3-5 times the applied mixture strain. Some regions within the mastic, however, experienced strain levels as high as 30 times the applied strain. Micromechanics analysis has shown the binder strain to be between 3-5 times that of the mastic. The HMA shear modulus, as a function of strain level, was calculated using the model and compared with experimental measurements. The results showed that the binder high strains in the microstructure could contribute to the nonlinear behavior of the mix. However, the measured stiffness nonlinearity was more significant than the values predicted by the model. The experimental measurements showed a reduction in stiffness between 45 and 55\% when the strain was increased from 0.01 to $0.1 \%$. However, comparable reduction in stiffness was obtained in the model when the applied strain was increased from 0.01 to $1 \%$.

\subsubsection{Modeling Pavement Structure with ABAQUS}

Abaqus Standard analysis is a general-purpose analysis product that can solve a wide range of linear and nonlinear problems involving the static, dynamic, thermal, and other response of components. Abaqus/CAE [3] allows models to be created quickly and easily by producing or importing the geometry of the structure and decomposing it into meshable regions. Physical and material properties can be assigned to the geometry, together with loads and boundary

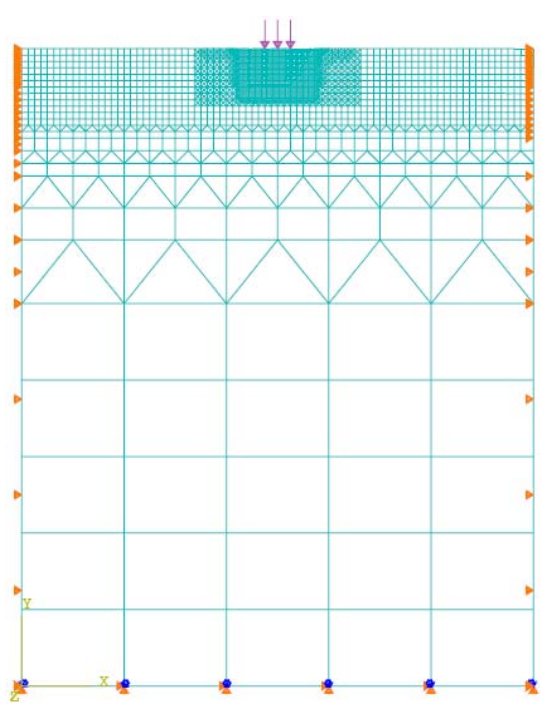

a. Single load case

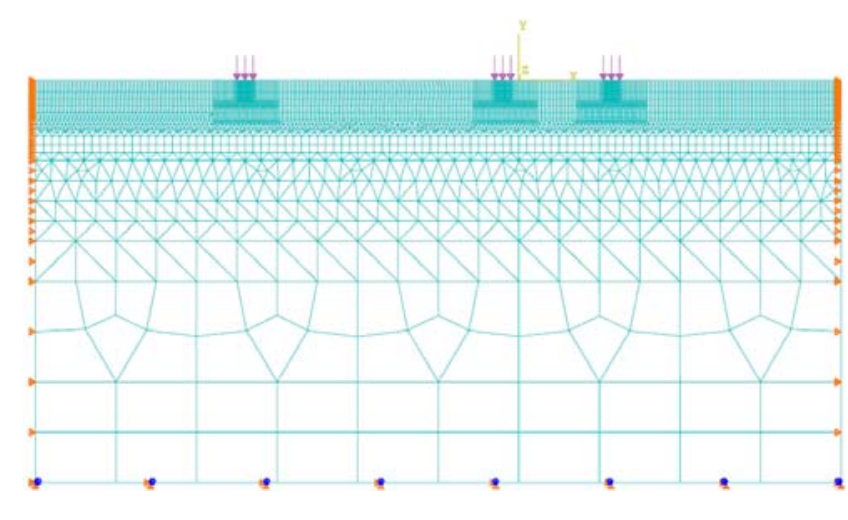

b. Multiple load case

Figure 6.12 FE 2D model with single and multiple load cases

conditions. Once the model is complete, Abaqus/CAE can submit, monitor, and control the analysis jobs. The Visualization module can then be used to interpret the results. 
In this research, the pavement has been modeled from a single part where several regions have been created later by partitioning the part in order to assign different sections to the resulting regions which are used throughout the modeling process as well as to gain additional control over the meshing generation process. The upper part of the model has been partitioned in to several smaller sections, each with a maximum of $50 \mathrm{~mm}$ depth in order to easily assign material section as well as to change the thickness of the structural layers. The intermediate and lower part of the model is partitioned with variable depths ranging from $100-1000 \mathrm{~mm}$.

In both 2D and 3D simulations, each model contains a single deformable solid part in a local coordinate system. The 2D continuum element is created by a shell feature in which thickness is considered small compared to the width and depth. The quadrilateral and triangular element types have been selected for smooth transition from courser to fine mesh.

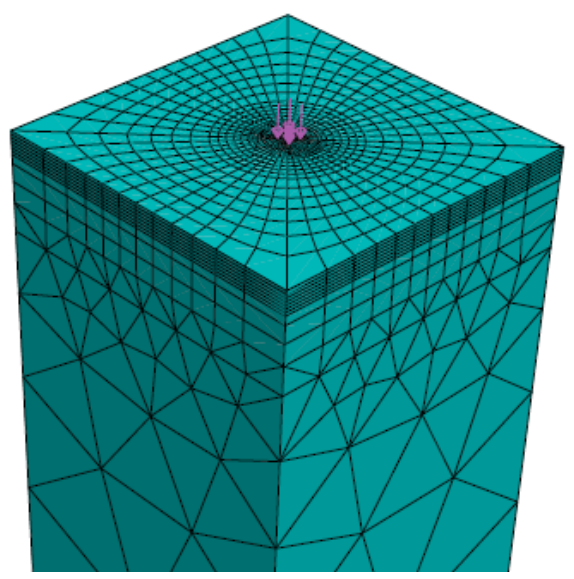

a. Model with reduced boundary effect

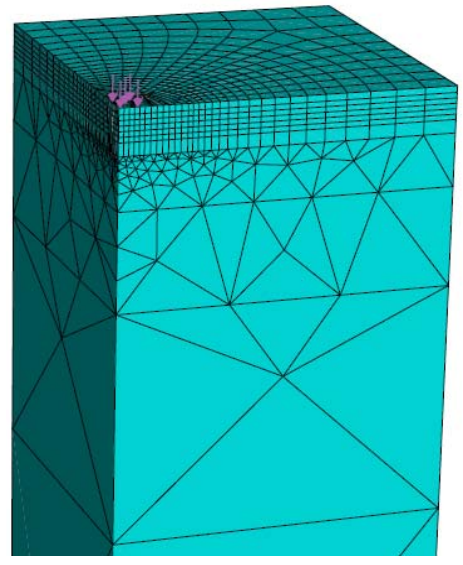

b. Model with axis symmetry

Figure 6.13 Abaqus 3D model

Different 3D model types have also been used for single and multiple load cases. An attempt has been made to reduce the effect of the boundary effect by applying load at the center of the

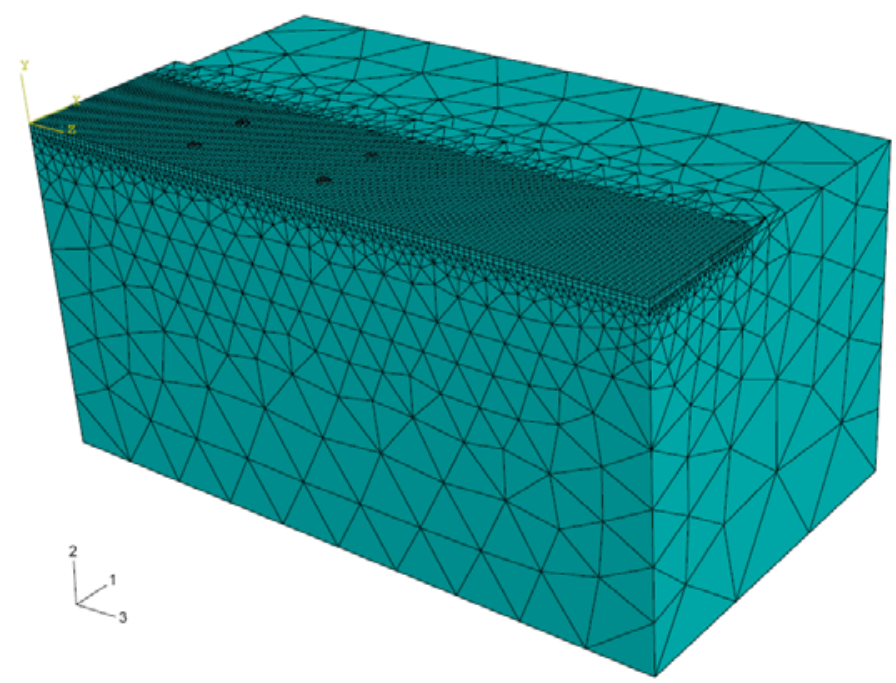

Figure 6.14 Abaqus 3D model with multiple load case

model sufficiently far enough from the boundary. In addition, in order to get advantage of reduced computational time and memory space requirements, axis symmetry has also been used in some models. 
Further more, single and multiple load cases have been simulated. It is clear that pavements are subjected to different patterns of vehicle loads ranging from single to tridem wheel loads with some heavy vehicles even having multiple wheel configurations, and hence the effects of multiple wheel loads have been simulated.

Different element types and sizes have been selected depending on the model type and according to the vicinity of the element with respect to the point of load application. The mesh density is controlled and managed by properly seeding the required area or edge of interest. In reality, the pavement layer thicknesses are very small relative to the width and length of the road and hence require selection of appropriate mesh density in order to get good result. The quality of meshing has greater influence on the output results. Of course, very fine mesh throughout the model is time- and memory consuming, and hence, an optimum meshing is required. Therefore, a very fine mesh (the minimum dimension is about $10 \mathrm{~mm}$ ) is assigned at the contact area and around it and such meshing continues till mid depth of the subbase course as shown in Figure 6.12 through 6.14. This meshing technique is used to refine the approximation around the contact area and in AC layers as well as to obtain smooth stress-strain contours. Meshing becomes courser at lower depths and/or elements which are far from the point of load application.

In order to assign material properties to a part or a region of part, a section which contains information (mostly refer the material name) about the part/region has to be defined. About five section properties have been created in both 2D and 3D models to represent the wearing, binder, base, subbase and the subgrade layers. Then the properties of a material have been assigned to the region of the model through the section referring to a particular material definition.

\begin{tabular}{|l|l|l|l|l|}
\hline $\begin{array}{l}\text { Material } \\
\text { Type }\end{array}$ & Layer & E (Gpa) & $v$ & $\begin{array}{l}\text { Temperature and } \\
\text { Shift factors }\end{array}$ \\
\hline \multirow{4}{*}{ viscoelastic } & Surface course & 2.00 & & $\theta=30^{\circ} \mathrm{C}$ \\
\cline { 2 - 3 } & Binder course & 10.00 & 0.30 & $\begin{array}{l}\mathrm{C}_{1}=17.44 \\
\mathrm{C}_{2}=52.10\end{array}$ \\
\cline { 2 - 3 } & Base course & 5.00 & & NA \\
\hline \multirow{3}{*}{ Elastic } & Subbase course & 0.600 & \multirow{2}{*}{0.35} & \\
\cline { 2 - 4 } & Subgrade & 0.045 & & N \\
\hline
\end{tabular}

Table 6.2 Typical material input

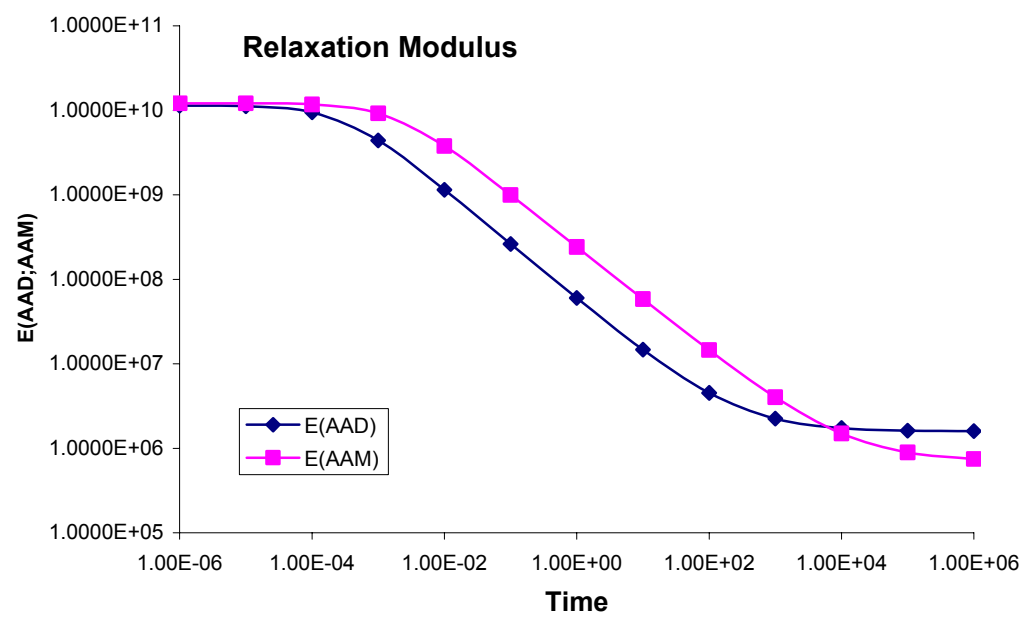

Figure 6.15 Material parameters for viscoelastic analysis 
The basic geometry of the pavement structure consists of the asphalt concrete (wearing, binder and base courses), the sub base and the subgrade layers. It is assumed throughout the analysis that the AC layers rest over a sound substructure of subbase and subgrade layers in such a way that the deformation is dominantly accumulated with in the AC layers; therefore both the subbase and the subgrade materials were assigned elastic material properties throughout the analyses. On the other hand the asphaltic layers are viscoelastic in nature and subjected to high wheel load which lead the AC layer to under go time dependent deformation therefore they are assigned elastic and viscoelastic properties under different circumstances. Some material properties assigned to sections at different computational circumstances have been outlined in Appendix B.

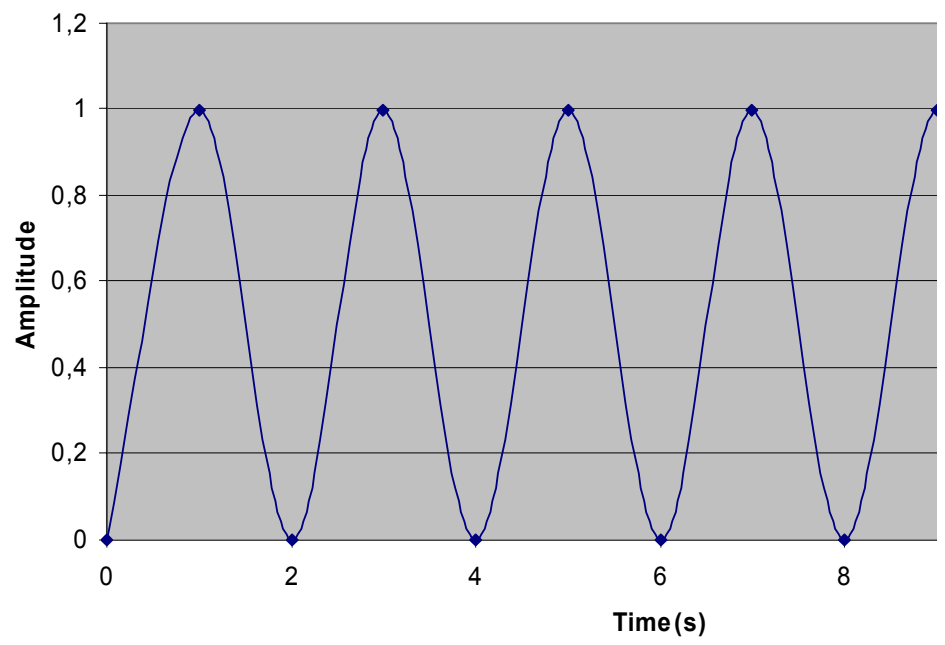

Figure 6.16 Amplitude definition

Within a model, sequences of analysis steps have been defined which have provided a convenient way to capture changes in the loading and boundary conditions. Load is a step dependent parameter which is defined in the required analysis procedure. A load of an equivalent contact pressure between the wheel tire and pavement surface with specified amplitude has been applied on the selected region over a circular/rectangular surface area (in case of 3D) and on a line equivalent to the estimated tire contact length.

The boundary condition greatly influences the analysis result. In reality, the width of a pavement is very short relative to the length; however it is constrained by the shoulder and/or adjoining land. The boundary condition has been set by taking into these facts. Therefore, except in the case of axis symmetry, the boundaries in the vertical faces have been constrained from

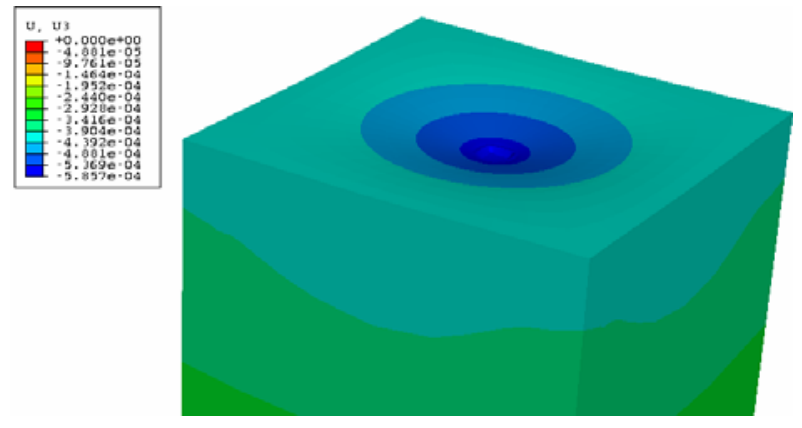

a. Deflection output

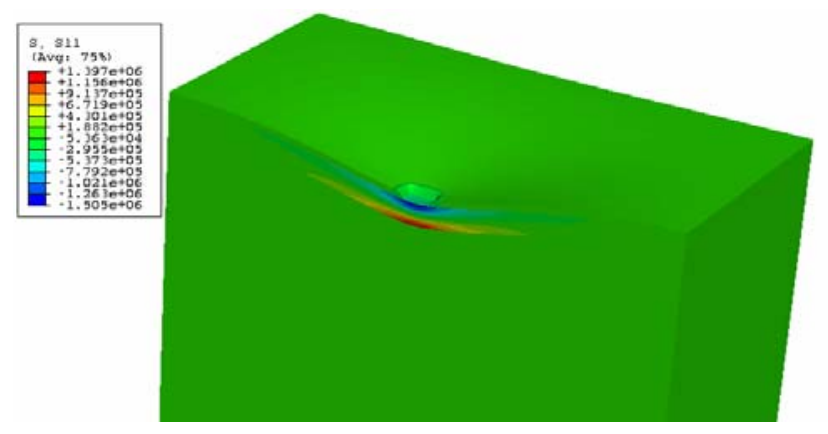

b. Lateral stress output

Figure $6.17 \mathrm{FE}$ field output in the visualization module 
displacement normal to the face and rotation through the vertical axis. At a depth sufficiently below the subgrade, in order to avoid/reduce the effect, a fixed boundary condition has been assumed in both rotation and translation. These assumptions might have their own impact on the results unless thorough precautions have been taken. To avoid such effects sufficient distance has been left between the point of load application and the boundary of the model.

Result information from the output database has been analyzed from various angles. Besides the graphical display, selected field output data has been further analyzed and compared with respect to a particular reference.

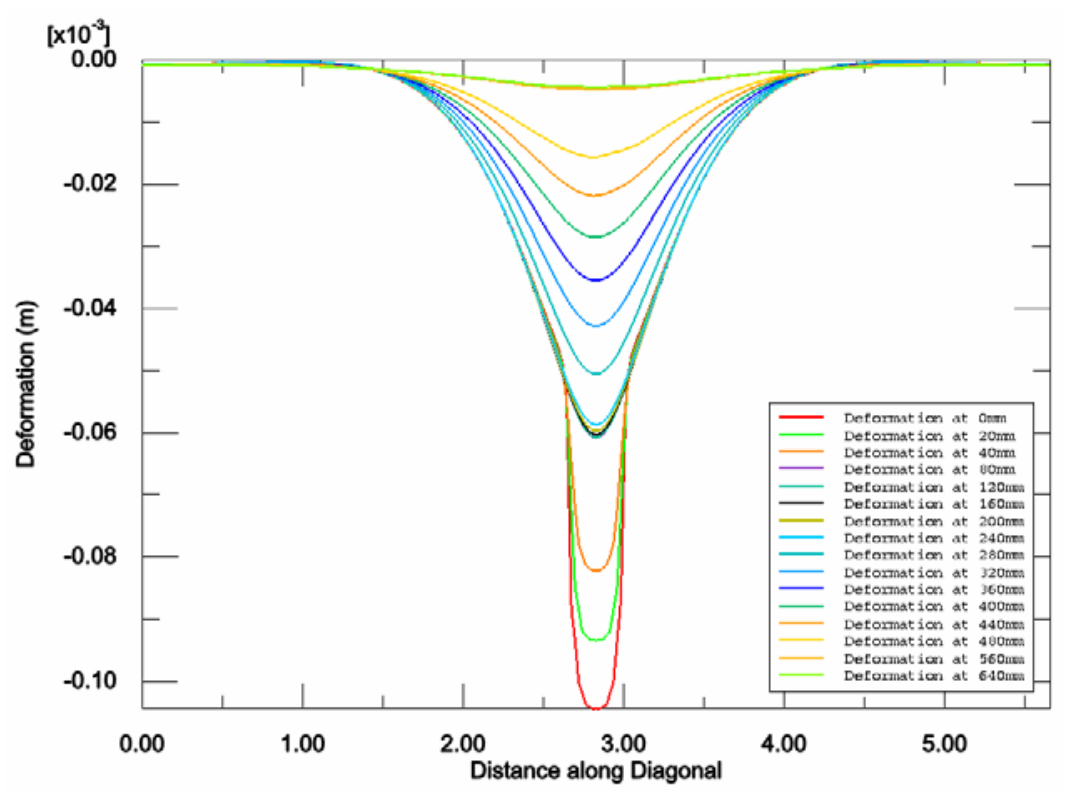

a. Deformation output

Strain Variations

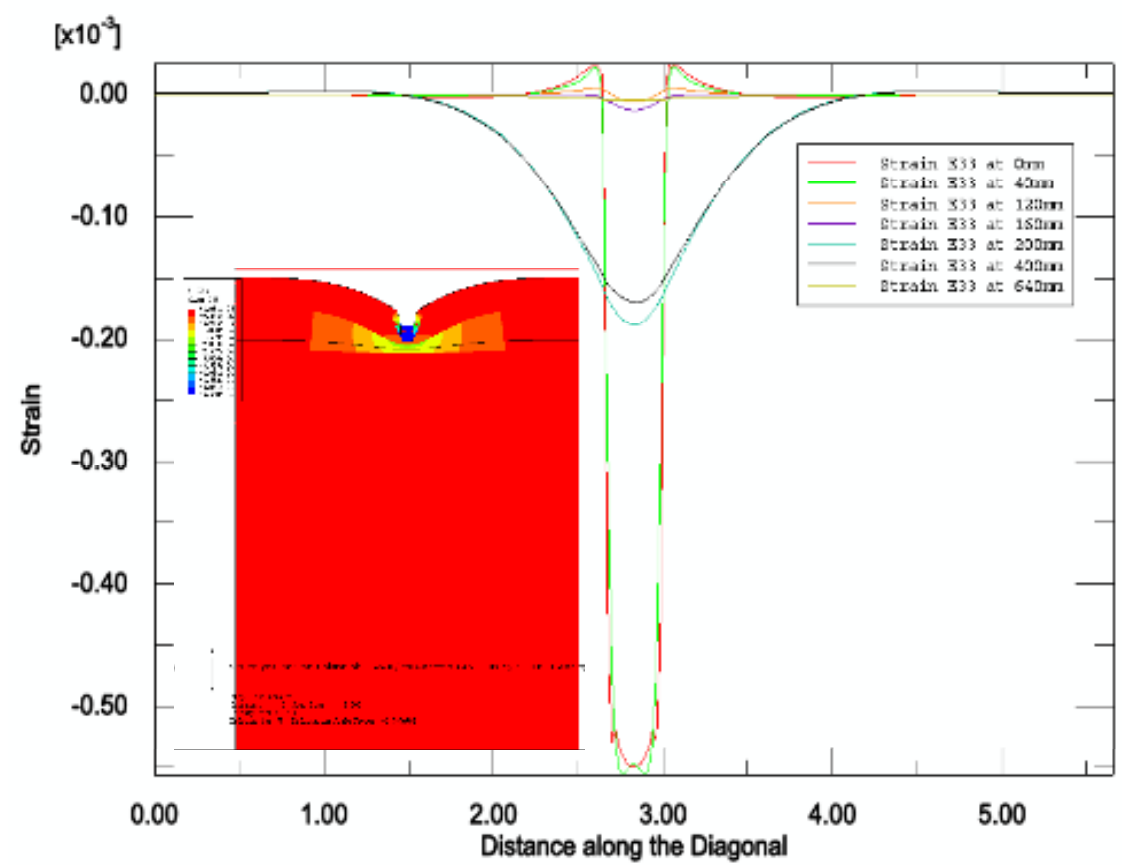

b. Strain output

Figure 6.18 Result analysis with reference to selected path 
Under some circumstances, results have been post processed with other program (such as in MATLAB and Excel) in order to refine the analysis. Of course, some of these outputs have been used as an input for rut prediction model which is explained in the next chapter.

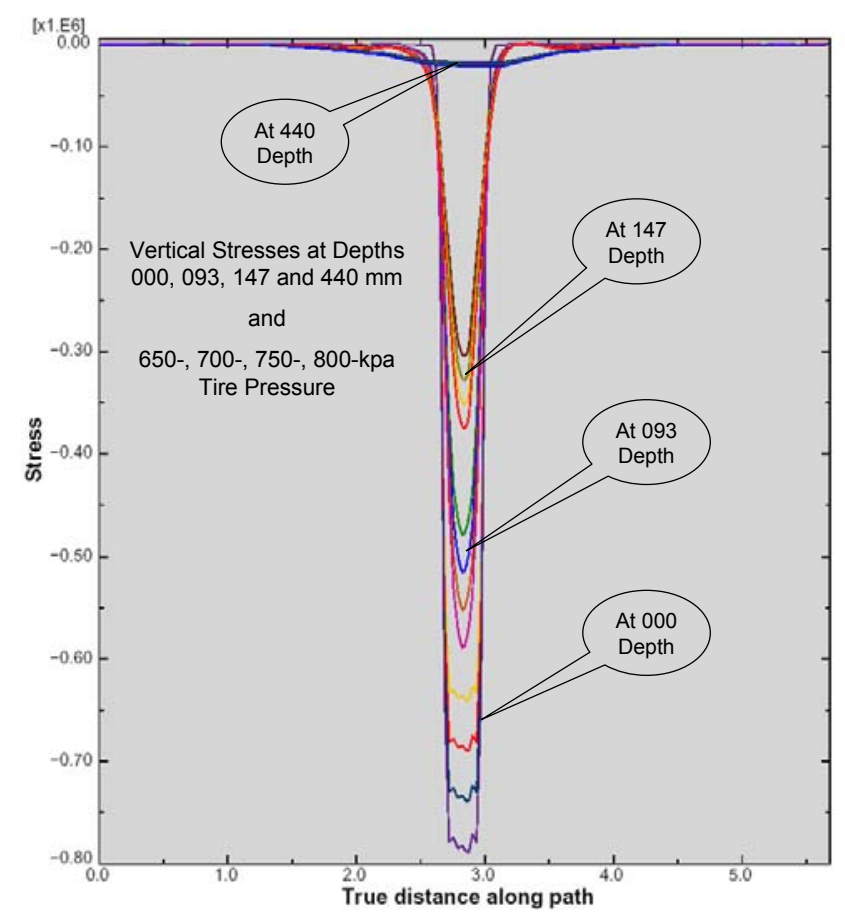

Figure 6.19 Output analysis with respect to applied pressure at selected depths 


\section{FE Result Analysis and Rut Prediction Model}

The FE analyses mainly have focused on the stress-strain state of AC pavement with varying loading conditions, structural geometries and material properties. 2D and 3D finite element models have been created and analyzed to evaluate the stress-strain components at critical locations where the damage is believed to initiate. The pavement has been layered in 5 sections: the wearing, binder and base courses which have been assigned with elastic and viscoelastic material properties at various computational circumstances. The subbase and subgrade materials have been assigned elastic properties in all analyses.

In reality the road is subjected to different types of load with high number of such load applications even per day. When such loading conditions are extended throughout the service life, the pavement analysis requires huge amount of resources. Even at this information age it is found difficult to simulate and analyze the actual pavement behavior due to the complex nature of the time dependent characteristics of loading, material properties and environmental conditions.

Rut formation is material, load and environment dependent damage parameter which accumulates for every single load pass along the wheel track. The type, magnitude, distribution and timing of loading are quite very vast and complex. Material and structural properties as well as their response for various loading conditions depend on their inherent characteristics and the environment they are exposed to. The environment, especially temperature in which it severely affects the material and structural responses, varies with respect to time of a day and season of a year. A pavement is exposed to such kind of complex and interrelated parameters and undergoes to permanent deformations through time. Interpreting the complicated interaction of various factors that the pavement is subjected to and formulating and modeling one of the most significant damages in the pavement structure during its service life is the main task in this research.

An attempt made to evaluate the damage accumulation with FE demanded huge time and memory space requirements. Therefore, because of the limitation of the FE software due to highmemory and computation-time demand and to simulate prevailing traffic and climatic/temperature/ change simultaneously, a Burger-model-based program has been developed and programmed to predict the accumulation of rutting along the wheel path during the presumable design period of the pavement under the prevailing traffic and temperature ranges. The critical parameters and associated influences as well as factors governing the deformation behaviors are discussed in subsequent topics. The pavement responses under various loading, material properties and pavement structural geometries are extracted from FE analyses. The analysis results and relevant notes are discussed below.

\subsection{Finite Element Analysis}

The finite element method is capable of simulating responses of pavements subjected to axle loads with different tire pressure and multiple wheel configurations. The primary response parameters of a pavement necessary for damage prediction model are evaluated by employing FE method. These parameters include the stress-strain states in both sub and superstructures and deflection at the surface of a pavement.

In the finite element modeling of asphalt concrete, the result output is extracted from the corresponding job.odb files and post processed to get the required pavement response. The influence of materials properties, variations of structural geometries and single and multiple wheel load assemblies over the various primary pavement responses have been evaluated.

It is found worthfull here to discuss some important pavement responses obtained from FE analyses. The deflection behaviors as well as normal, shear and lateral strain-stress states have been evaluated at presumable critical positions in the model. In addition, for comparison 
purpose, supplementary result analysis outputs are evaluated on selected points and described below.

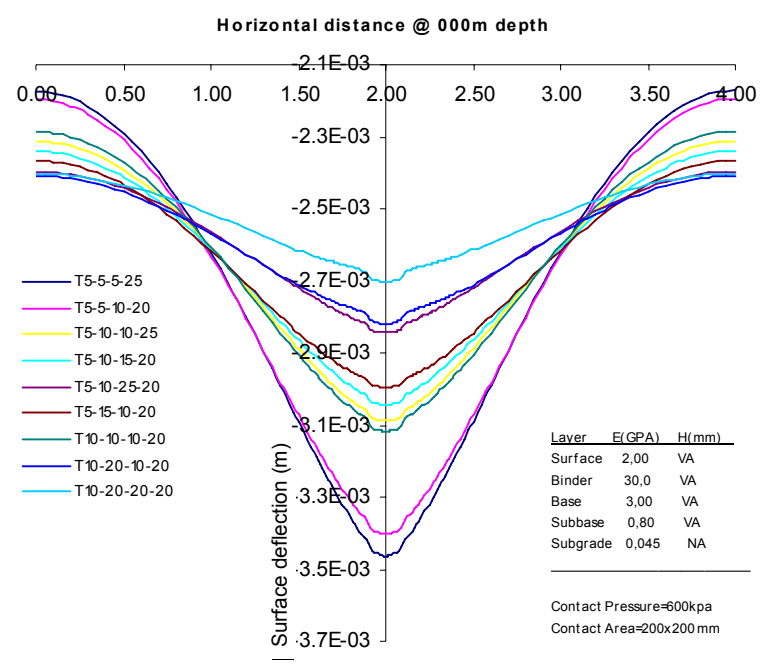

Figure 7.1 Instantaneous deflection behavior with change in AC thicknesses

The deflection behavior of a pavement throughout the service life under different load, material and structural properties is one of the major issues in pavement analysis and design. Even though the instantaneous deflection is not the main concern in this research, it is important to show how it is affected with various parameters. Figure 7.1 shows the effect of thickness (the designations T-5-10-15-20 represent thickness of 5, 10, 15, and $20 \mathrm{~cm}$ of wearing, binder, base and subbase course, respectively) on the instantaneous deflection. The comparison is made with respect to contact pressure, change in E-Moduli and thicknesses of the layers. The analysis shows that the instantaneous deflection obtained at top surface, contributed by all subsequent layers, has increased obviously with load. The deflection, in general, has decreased with increasing the E-modulus of the asphaltic layers and layer thicknesses. Doubling the total AC layer thicknesses from 150 to $300 \mathrm{~mm}$ yields more than $10.9 \%$ reduction in surface deflection. In addition, the deflection gets smaller with increasing the layer thicknesses; for example increasing the base course thickness significantly reduces the surface deflection. Relatively, higher Elasticity modulus intermediate layers of binder and base course bears lower instantaneous deflection. The change in the magnitude of surface deflection is rather sensitive to change in Young's modulus of the base course than the wearing course.

As it is clearly known, the main stress-strain states responsible for permanent deformation on the pavement are the development of vertical compressive and shear stresses within the structural layers. Rutting is formed when the pavement under the repetition of traffic loadings consolidates and/or there is a lateral movement and reorientation of the materials in the AC layers. The lateral movement is a shear failure and generally occurs in the upper portion of the pavement surface. It is obvious that the vertical stress within the asphaltic layer is maximum compared with subbase and subgrade layer and the magnitude of the stress developed with in each layer is proportional to the applied tire pressure on the surface of the pavement. The FE analyses with a single wheel load have revealed that the vertical stress developed at the surface is greatest as shown in Figure 7.2 through 7.4. Vertical contact pressure of 600 and 500 kpa are applied on model represented by Figure 7.2 and 7.3, respectively. The maximum vertical compressive stress is developed in the wearing layer and rather confined within the wheel foot print. This phenomenon is also observed through the AC layers of the pavement. In the wearing course, the vertical stress just outside of the contact area becomes smaller and vanishes after a couple of centimeters as shown in Figure 7.3. On the other hand, there is a significant drop in magnitude of this stress at lower depths (at subbase and subgrade layers), however, the 
influence area gets wider down the pavement depth. This does not mean the strain also show the same trend, rather the maximum vertical strain is observed at the top of the subgrade, (which is evidenced also in many literatures [Archilla and Madanat 2000, Saad et al. 2005]). This phenomenon is due to less stiffness/lower E-value/ characteristics of the subgrade layer. Relatively, the vertical strain within the intermediate layers (binder, base and sub base) is lower than both the wearing and subgrade layers because of higher vertical stress with in the wearing course and higher elasticity modulus used in both binder and base layers.

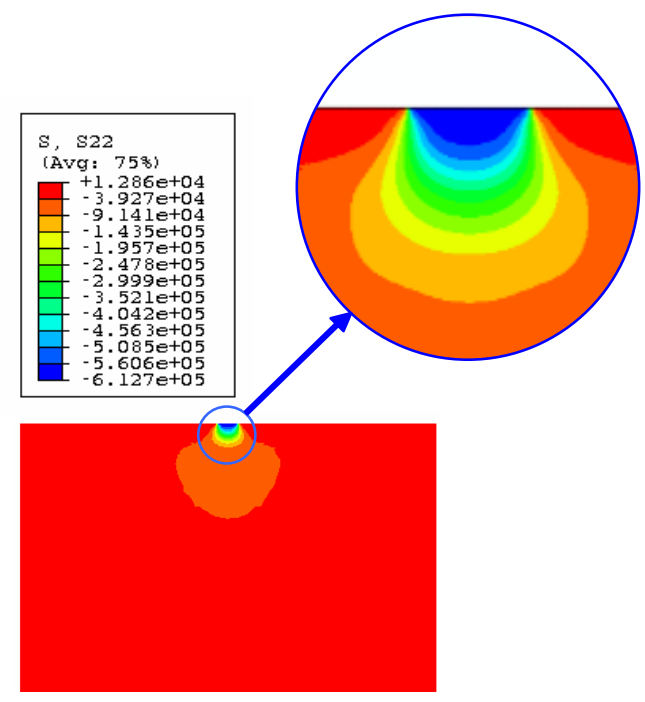

a. FE vertical stress distribution

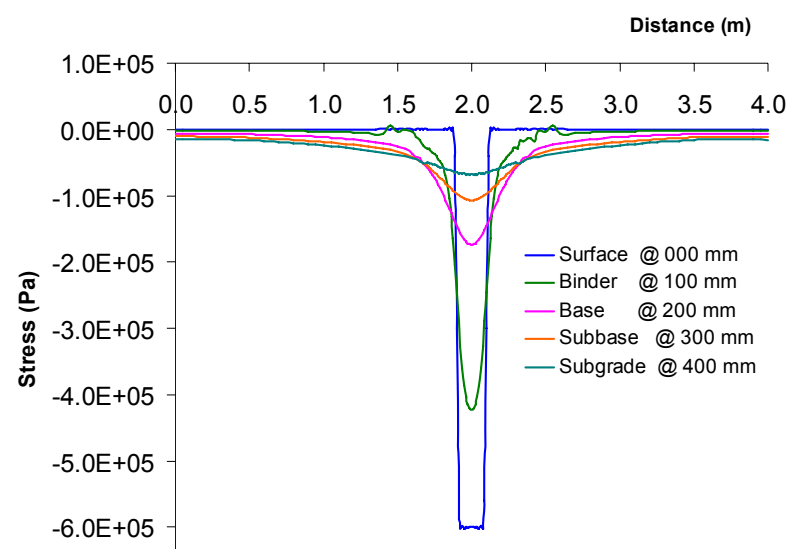

b. Vertical stress on selected horizontal path

Figure 7.2 FE vertical stress out put in AC layer

Figure 7.3 shows the vertical stress along selected vertical paths in the model starting from the center of load application. The paths are spaced at $50 \mathrm{~mm}$ interval as indicted. At the center of the wheel print, the wearing course experiences the maximum compressive stresses with the magnitude almost close to the applied contact pressure. The stress gradually decreases with increase in depth but with a wider area of influence as can also be shown in Figure 7.2 (b) and 7.3.

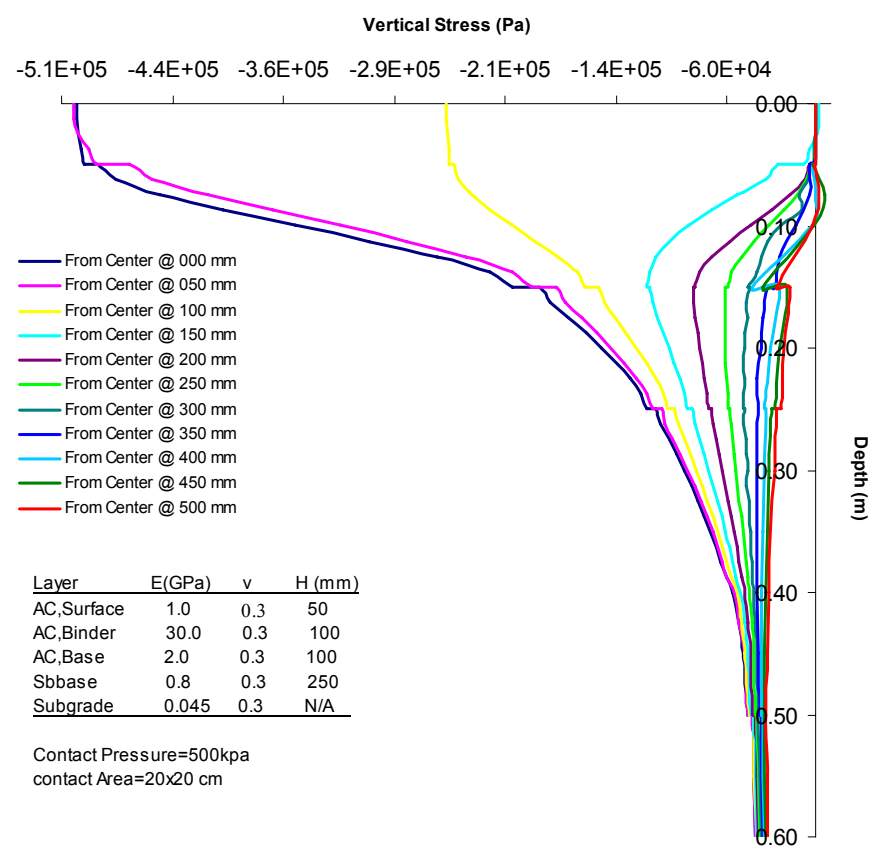

Figure 7.3 Vertical stress distribution on selected vertical path from centre of load application 
The structural properties of each layer have a significant role on the performance of the pavement. Among which the elastic modulus of each layer has predominant influence on the materials' stress-strain behavior. The analysis carried out for different E-moduli for the asphaltic layers reveals the same idea as shown in Figure 7.4. As indicated in Figure 7.4 (a) increasing the binder E- modulus shifts the curve to the right, i.e., decrease in the magnitude of the vertical stress developed within each layers. Keeping structural geometry, loading condition and other material parameters constant, an increase the E-moduli of the binder twice yields $4.9 \%$ and $14.3 \%$ reduction in vertical stresses developed at the mid-depth of the binder and base layers, respectively, just directly below the point of load application. In addition, increasing the same by forth fold yields as well $11.1 \%$ and $27.6 \%$ in these layers. However, there is a slight increase in stress in the wearing layer, in both cases. This holds true as well in case of increasing the Emoduli of the base course, there are increase in stresses developed on both wearing and binder courses. These imply that an increase in E-moduli reduces stress at lower depths and increases the same slightly in upper layers.

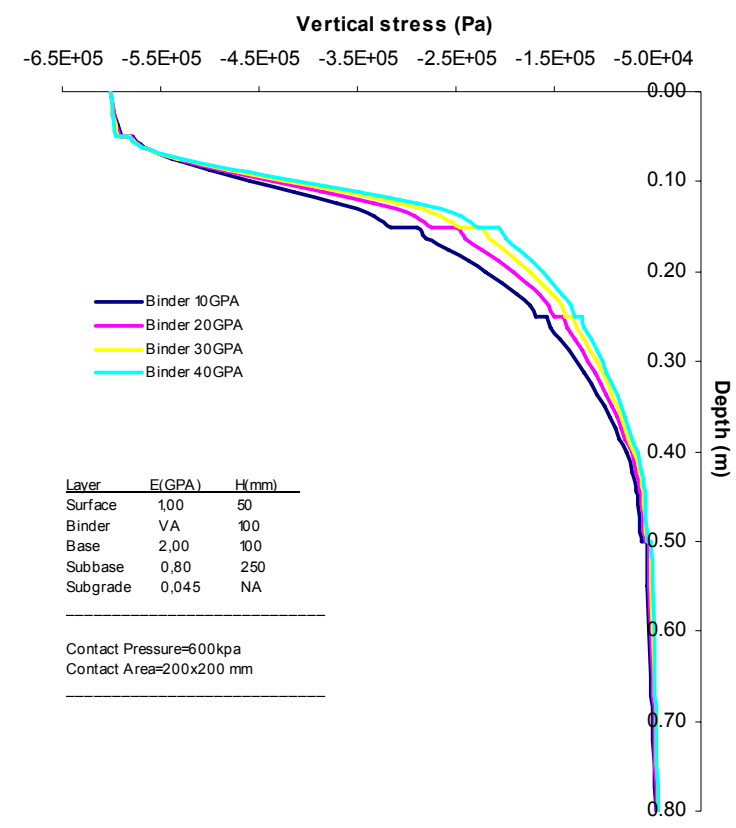

a. Vertical stress with varying Binder E-Moduli

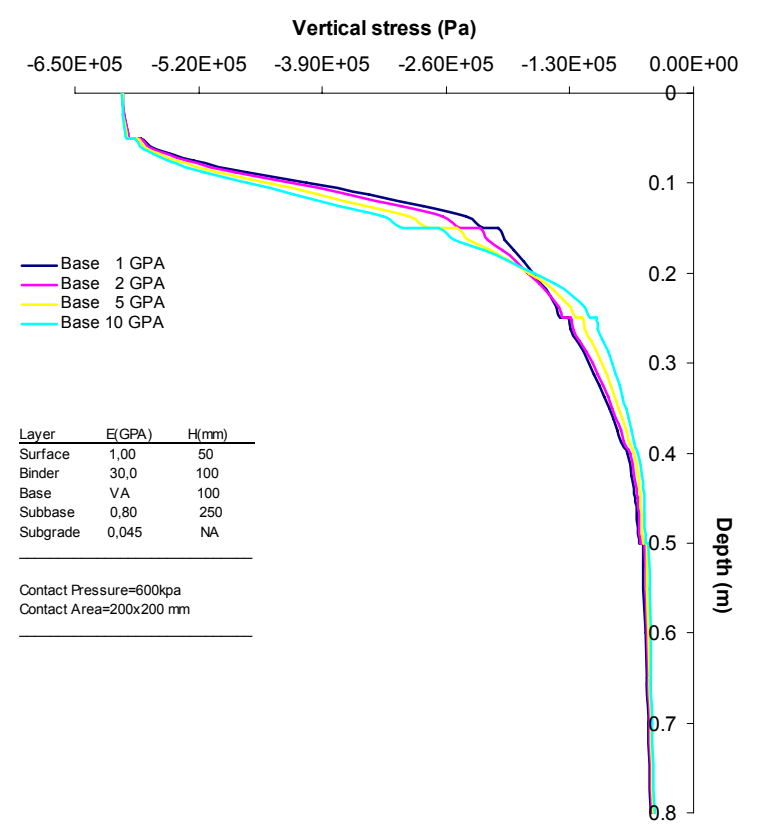

b. Vertical stress with varying Base

E-Moduli

Figure 7.4 Vertical stress variations w.r.t. different binder and base E-modulus

On the other hand, the variations of the elasticity modulus of the wearing course are rather pronounced in change in the stress-strain states of the wearing course itself and do not have major influence on the stress-strain state of the lower layers (refer Appendix C-7). There is a reduction in vertical and shear strain and an increase in lateral stress with in the wearing course with higher E-value. It clearly indicated from the analysis that the strength of the asphaltic layers is in favor of the subbase and subgrade layers in decreasing the stress-strain states.

The other most important factor which affects the stress-strain state of a pavement is the structural layer thicknesses. Unlike lateral and shear stresses, higher AC layer thicknesses experienced slightly higher vertical stresses, how ever, it has been observed that there is a reduction in the vertical strain due to increase in layer thicknesses. 


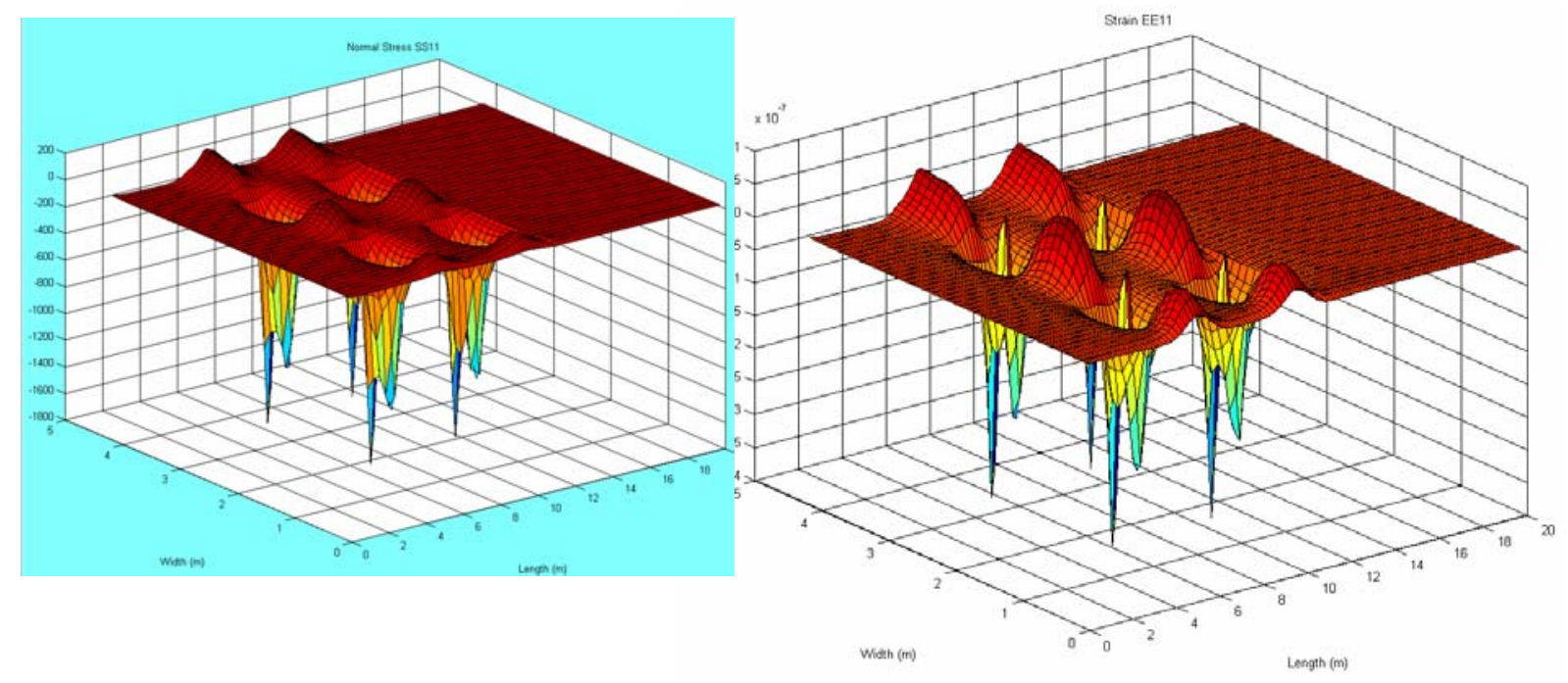

Figure $7.53 \mathrm{D}$ lateral stress-strain state due multiple wheel loads

A 3D finite element analysis confirmed also that the vertical stress-strain magnitude is rather high in AC layer depths directly below the wheal foot print..

Rutting of asphalt concrete pavement is caused by many factors. It is considered that shear stress is one of the most significant contributors for the deformation and up heel along and just on the sides of the wheel path. The FE analysis shows that the binder layer is subjected to remarkably high shear stress as shown in the Figure 7.6 with the maximum magnitude just outside of the projected contact area.
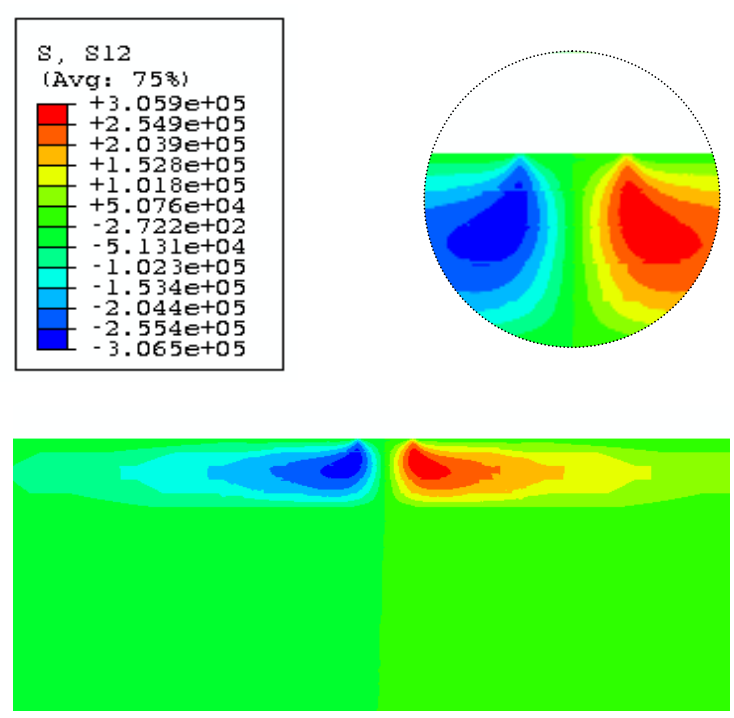

a. Model shear contour

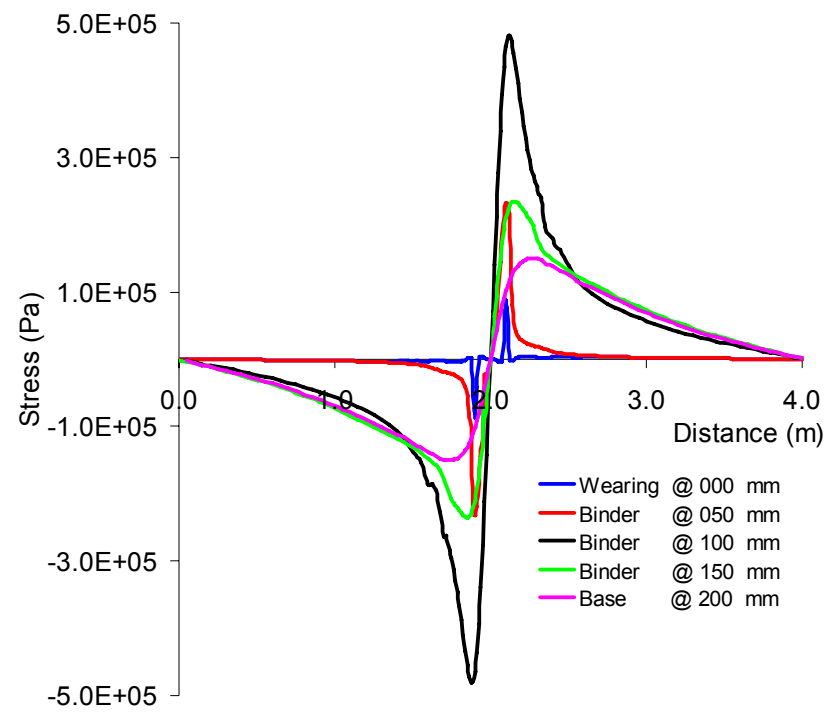

b. Shear stress diagram at selected horizontal paths

Figure 7.6 Shear stress in the pavement section

As indicated in Figure 7.6 and 7.7, critical shear stresses (for the given loading conditions, structural geometry and materials parameters) are within 50-200 mm depth below the surface, i.e. within the binder layer. In case of 3D analysis critical shear stresses are also observed close to the surface of the model a few centimeters away from the wheel print. 


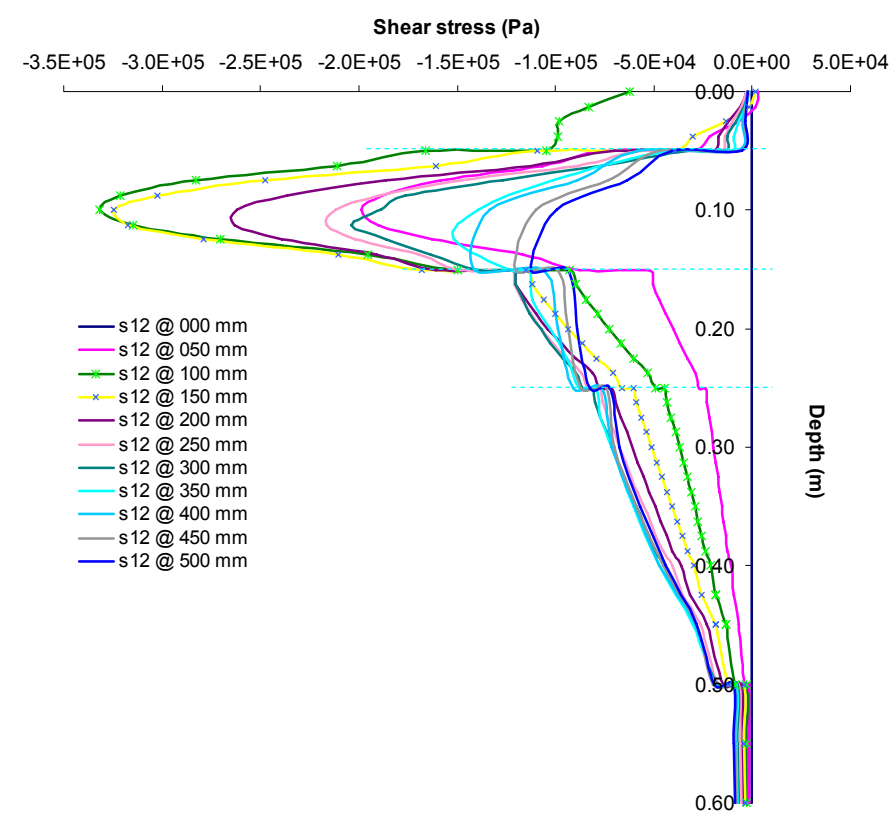

Figure 7.7 Shear stresses variations in selected vertical path

As it is clearly indicated, higher elasticity modulus in the binder course rather increase the shear stress developed with in the binder layer and decreases the same stress with in the base course and vise versa. The shear strain, in both cases reduces with increasing the E-moduli of the layers. The thickness of the layers plays constructive role in bringing down both shear stress and strain in the AC layers.

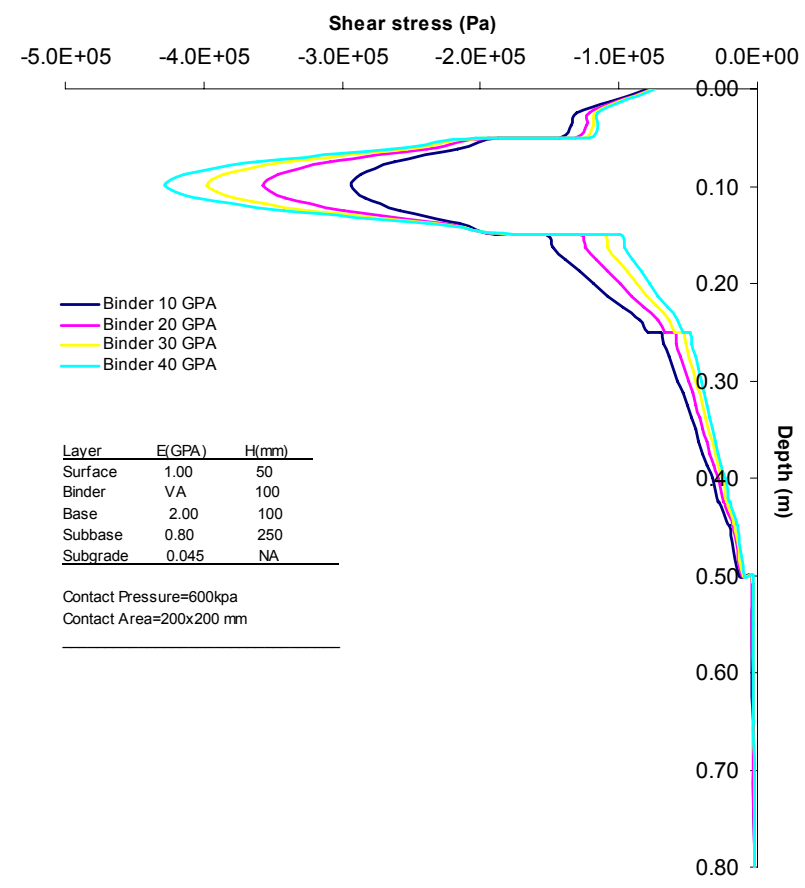

a. Shear stress with varying binder E-moduli

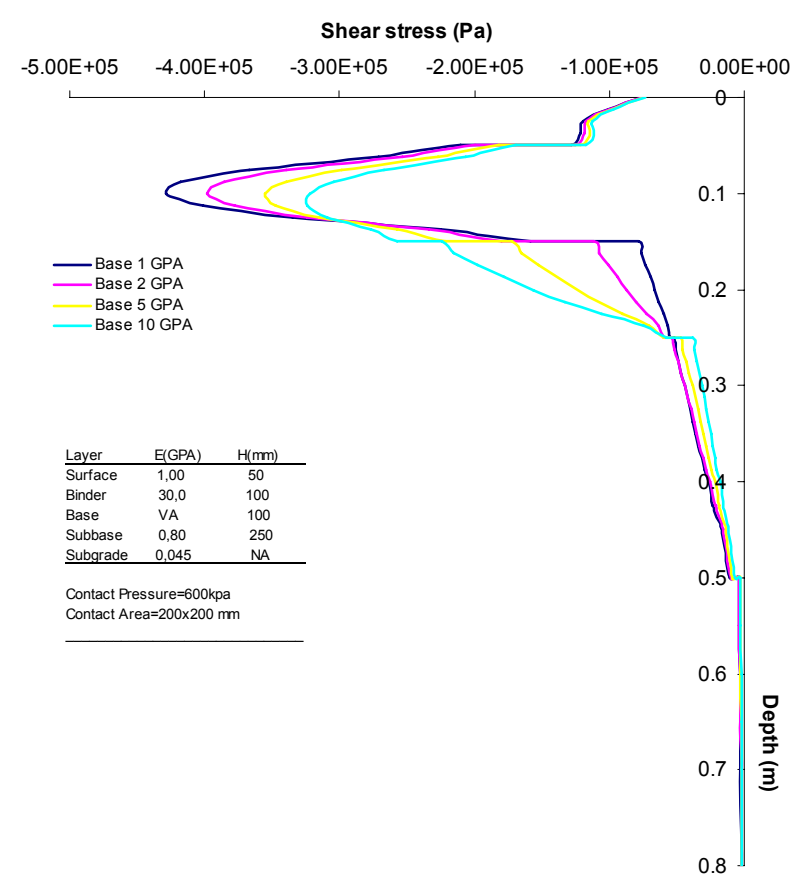

b. Shear stress with varying base E-moduli

Figure 7.8 Vertical stress variations w.r.t. different binder and base E-modulus

In this study, pavement responses due to single and multiple wheel loads have been evaluated in 2D and 3D FE models. The vertical stresses developed on the top layer are dependent neither the 
number of wheels nor their configurations. However, these stresses get overlapping some distance below the surface in case of multiple wheel loads. The magnitude and the area of influence depend on arrangement and the configuration of wheels. It is obvious that the magnitude of vertical stress developed at lower depths is by far greater than the one produced by a single wheel and dependent on the spacing of the wheels and axles.

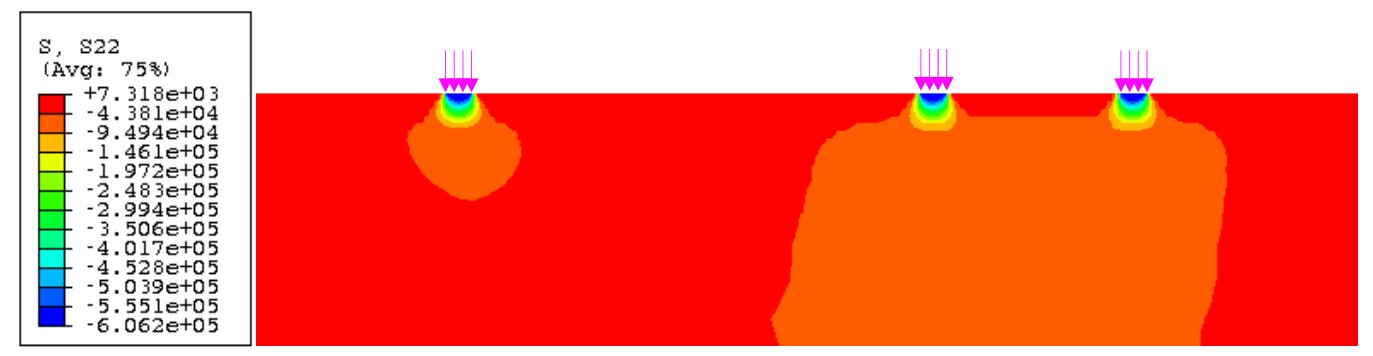

Figure 7.9 Stress state under dual wheel load application

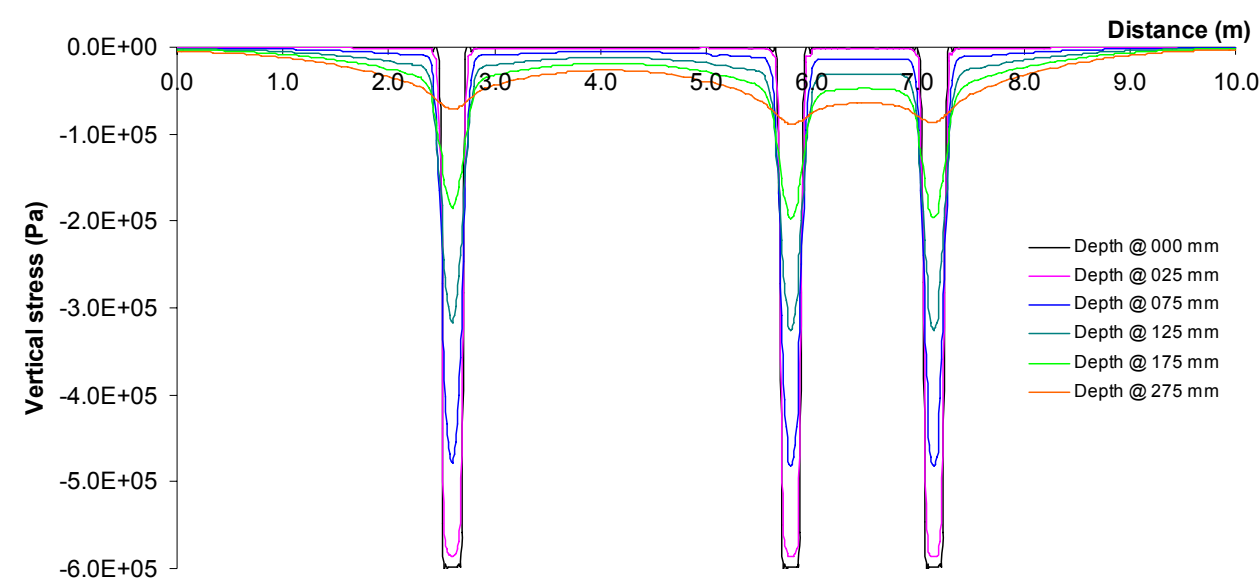

Figure 7.10 Influence of the tire configuration on the distribution of vertical stresses

As indicated in Figure 7.9 and 7.10, the magnitude of the stress in the wearing course is higher than the other asphalt layers and independent of the wheel configuration. The overlapping of stresses is gradually pronounced in the base course. Whether it is developed with a single load or multiple loads, the vertical stress developed at the top layers of the asphalt concrete is one of the major contributors for rut formation on this layer. Of course the rate of rut formation is a function of the frequency of such load application, materials properties and other external factors such as temperature and the rate of loading.

The analyses reveal that the subbase and subgrade layers suffer greatly with high stress-strain magnitude due to the influence of the overlapping of stresses developed by dual and tandem wheel assmbly which draws serious concern on reliable stability of these layers. Therefore it is very important to make sure that the materials used for these layers have to be strong enough so that the influence of such a phenomenon is within the bearing capacity of the layers.

As it is believed and universally accepted, the other stress state which significantly contributes rutting along the wheel path and up healing just on the side of the wheel track is the shear stress developed within the pavement structure [White et al. 2002]. The analyses have shown that the maximum shear stresses developed at some depth below the surface of the model. As shown in Figure 7.6a the maximum shear stress developed with in the binder layer around the periphery of the projected area. 
The same is true in case of multiple wheel load configurations a shown in Figure 7.11 below that the magnitude of shear stresses is higher on the binder course and the maximum magnitude developed at the front and rear wheels. This is due to the fact that higher shear get developed some distance below the surface and outside of the loaded area. As it is recommended by many researchers this layer is required to be more stiff and flexible so that the stress will remain within the capacity limit.

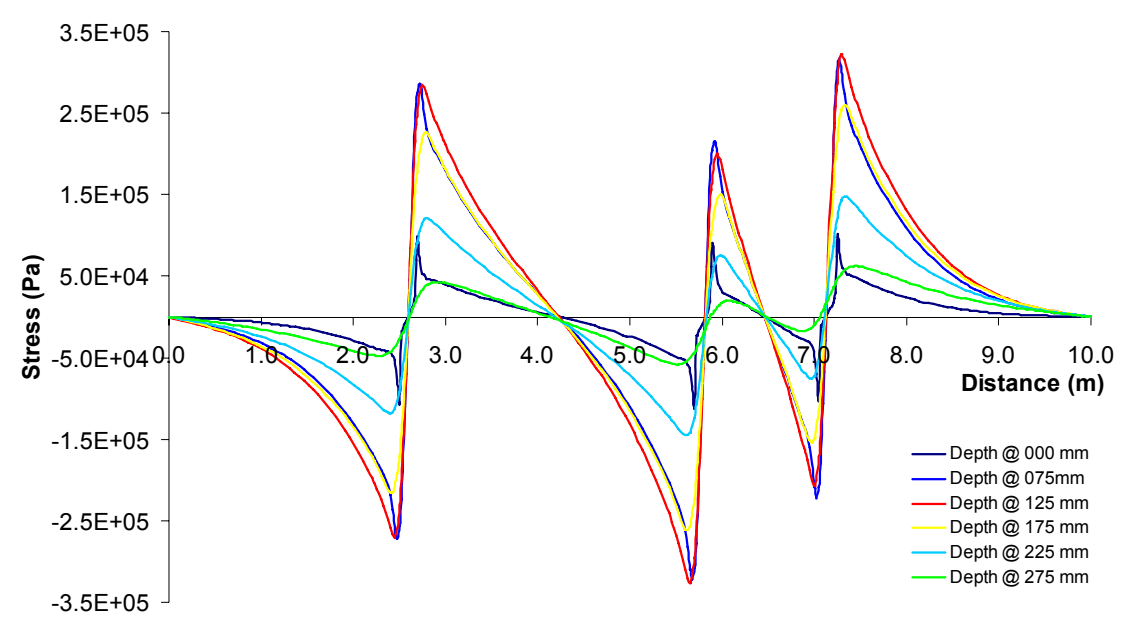

Figure 7.11 Shear stresses across the depth

Thus unless better quality of materials are provided on subbase and subgrade layers the permanent deformation can also be developed in these layers which ultimately accelerates the rutting progress of the pavement structure. The wearing course is subjected to high compressive stress; therefore quality of material required for this layer has to be carefully selected to avoid excessive damage in this layer as well as to reduce the abrasive effect of the wheel load.

As universally witnessed [Elseifi et al. 2006, Lee et al. 2000, Saad et al. 2005 and 2006], the tensile stress which is maximum at bottom of the base course, contributes the fatigue failure of a pavement structure. Similar to the vertical stresses, the magnitude of such stresses depend on the magnitude of the wheel load /contact pressure/, thickness, material property and temperature of the layers. The Abaqus analysis shows the same result as shown in Figure 7.12 and 7.13.

Because of relatively higher elastic modulus at the AC layers, the maximum tensile stress occurs at the bottom of the base layer. A dual wheel assembly affects the magnitude of the tensile stress/strain produced at the base course.

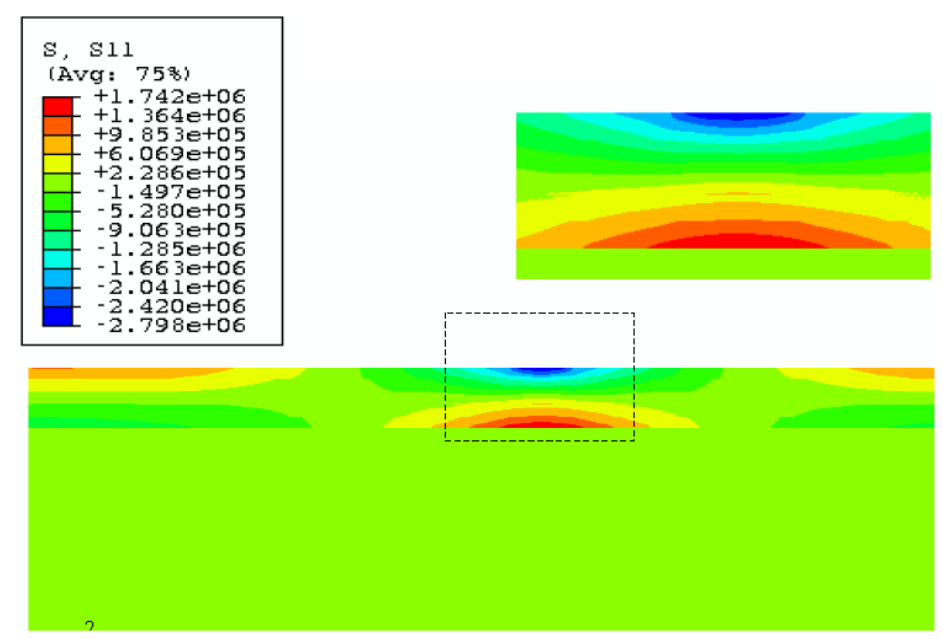


The area of influence due to tensile stress-strain at the bottom of the base course subjected to dual wheel is by far more than 1.75 times the area affected by a single wheel. This implies that the area of influence due to multiple wheel load configuration is very large which leads to high stress/strain development for an extended base area therefore the pavement would suffer greatly due to fatigue failure for multiple wheel load than a single wheel configurations. In addition portion of the material at the bottom of base layer may undergo relatively higher lateral deformation in case of multiple wheel load configuration.

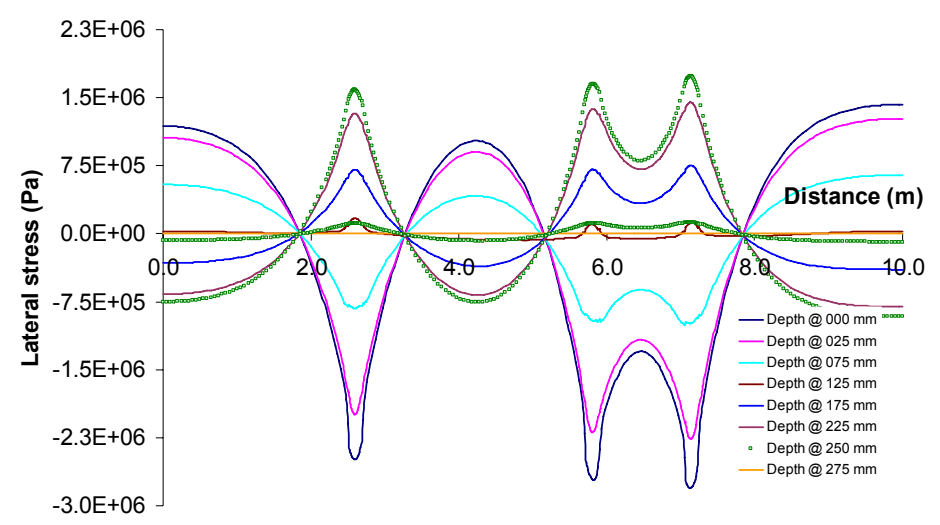

Figure 7.13 Horizontal stress due to dual wheel load

The other important factor considered in the finite element analysis is the influence of the structural geometry on the stress-strain states on a pavement. In general, thicker asphaltic layers significantly reduce the magnitude of stress and strains in the pavement structures, particularly for the substructure. More importantly the strain components are significantly decreased in thicker asphalt layers.

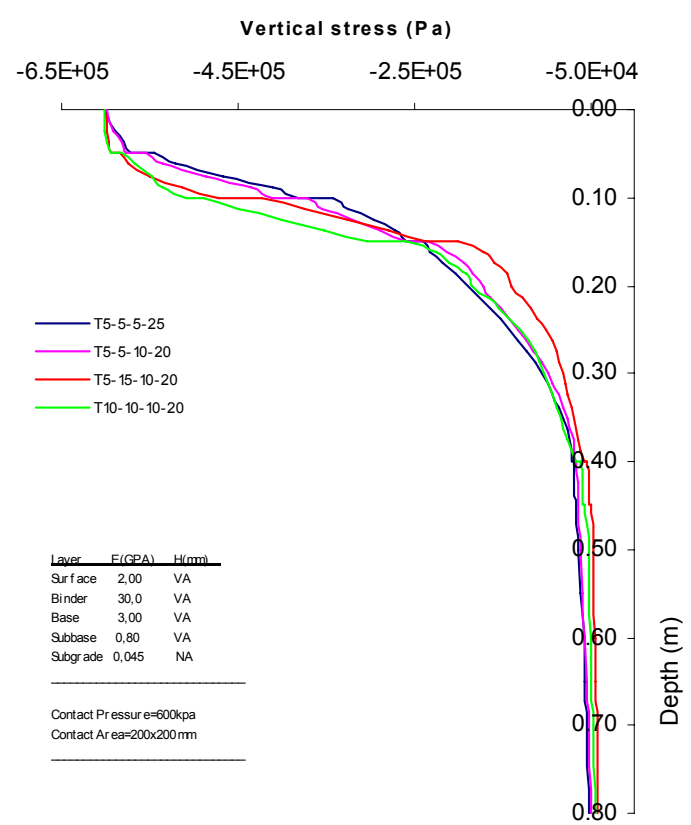

a. stress

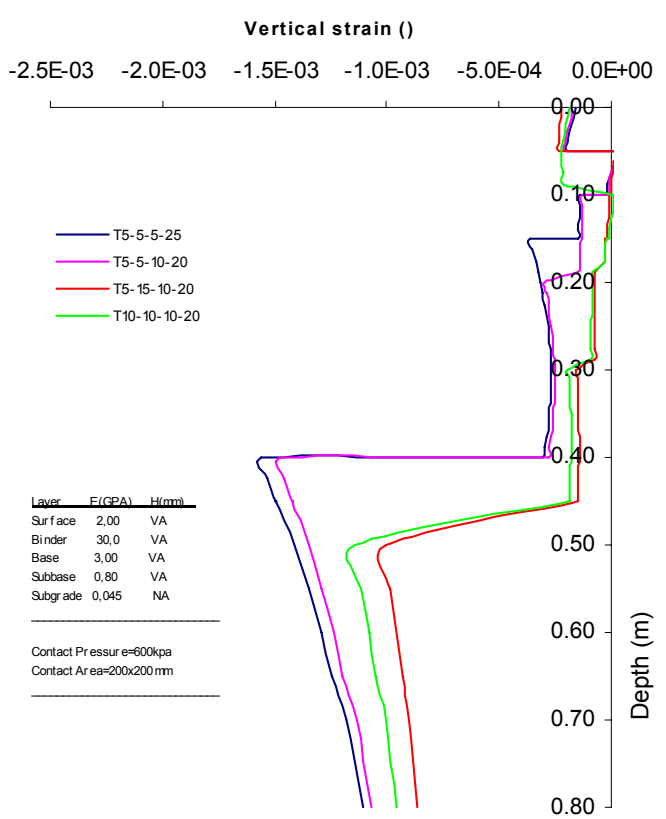

b. Strain

Figure 7.14 Vertical stresses and strains for different asphalt layer thickness

As can be seen from Figure 7.14 (a) above, at the mid depth of each layer, a reduced stress is obtained when the thickness is increased. The corresponding vertical stress is significantly 
reduced as shown in the figure above. The shear and the lateral stresses and strains show the same trend. As can be seen in Figure 7.15, the shear stress at the mid-depth of the binder layer decreased roughly by $25 \%$ when the asphalt layer thickness is increased from 150 to $300 \mathrm{~mm}$.

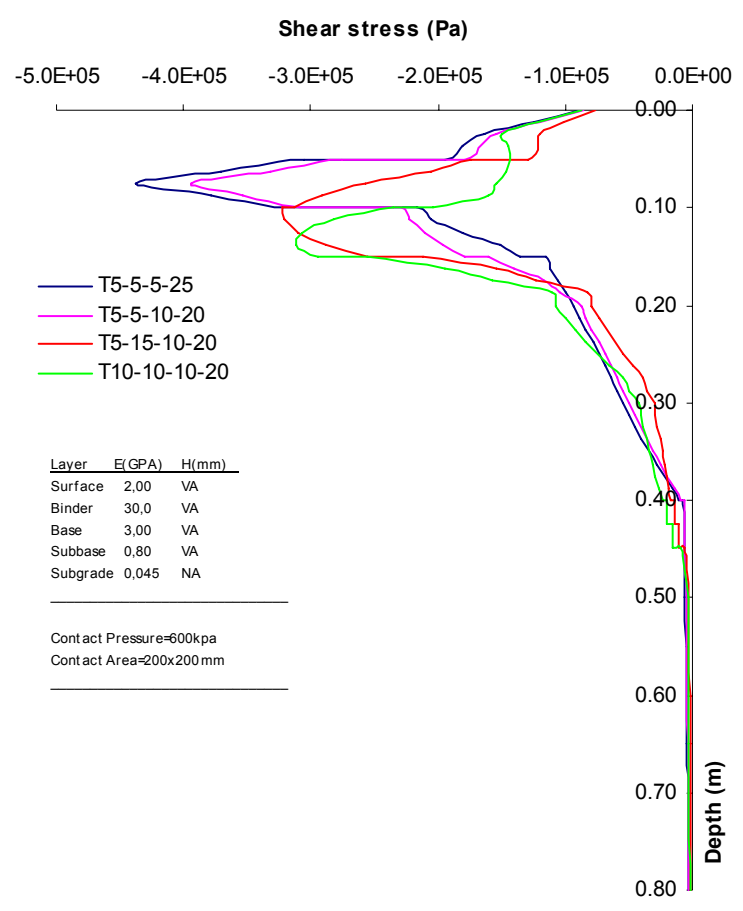

Figure 7.15 Shear stress for different asphalt layer thickness

\subsection{Rut Prediction Model}

The multitude of state of stresses caused by wheel loads result in multitude material responses that makes the damage accumulation process highly complex. Pavement structural damage especially permanent deformation depends on the material characteristics, the state of stressesstrains and the characteristics of the traffic load. The state of stress-strain in a pavement structure is not only dependent on the magnitude and configuration of the wheel load but also dependent upon the time and temperature dependent behavior of the constituent materials of the layer. The temperature gradient down to the depth has a direct influence on the viscoelasticity properties of the AC structures, and hence, it affects the response of a pavement when it is subjected to load.

The development of deterioration model plays an important role in designing and managing of road infrastructure. Numerically intensive simulations are required to model such complex loadings and material behavior interdependence. The ability to predict the magnitude and accumulation of permanent deformation or rutting in flexible pavements is an important aspect of pavement design. In order to take into account the effect of temperature on materials properties as well as to determine the deformation buildup of a pavement under different temperature and loading conditions, a rut prediction model is developed and programmed.

Since the rate of accumulation of permanent deformation is time dependent, the most suitable forms of constitutive model to simulate rutting is based either viscoelasticity or viscoplasticity. Accurate prediction of flexible pavement response requires the pavement temperature and the loading time because of their important role in characterizing the viscoelastic behavior of AC materials [Uzarowski 2006, Yin et al. 2008]. Therefore the transient temperature variance of pavement and timing of loading can not be ignored when analyzing the pavement rutting. This guarantees a more reasonable simulation analysis of rutting, much closer to the field situation and it will be more effective and powerful to address the rutting behavior. 
The stress-strain state is also dependent upon the materials parameters (elastic modulus and viscous parameter) which in turn depend on the temperature of the surrounding. Many researches [Nazarian and Alvarado 2006, Huang et al. 2008] have been undertaken to simulate the relationship between materials properties and temperature. The AC layers are exposed to surface therefore their property is greatly influenced by surface temperature. As discussed earlier the wearing course temperature is almost close to the air temperature where as temperature of subsequent layers relatively decreases with increasing depth. As a result the deformation behavior of the pavement is dependent upon the magnitude of the stress developed in these layers, materials characteristics and most importantly upon the temperature of the medium.

In many asphalt concrete modeling and design approaches as well as design standards, important parameters are either omitted or assumed for the sake of simplicity. For example the time dependent variation of traffic and associated flow characteristics are not taken in to account in many structural design standards. Several loading magnitudes are rather approximated by taking the equivalent standard axle load instead of evaluating the effect of wide range loading condition.

The impact of the environment has not been yet fully tackled. Of course, promising prediction models are currently emerging [Denneman 2007, Diefenderfer et al. 2006, Huang et al. 2008, Wellner and Kayser 2008, Yavuzturk et al. 2005] as far as temperature change, moisture effect and other environmental and climatic changes over time and an attempt has been made to analyze their influence on the performance of AC pavements over a period of time. The simultaneous occurrence of traffic loading at high temperature is totally out of the game in many standards.

Because of such limitations a pavement deformation model has been developed which aimed mainly on prediction of rut accumulation over a period of time due to time dependent mixed traffic flow as well as daily and seasonal temperature variations.

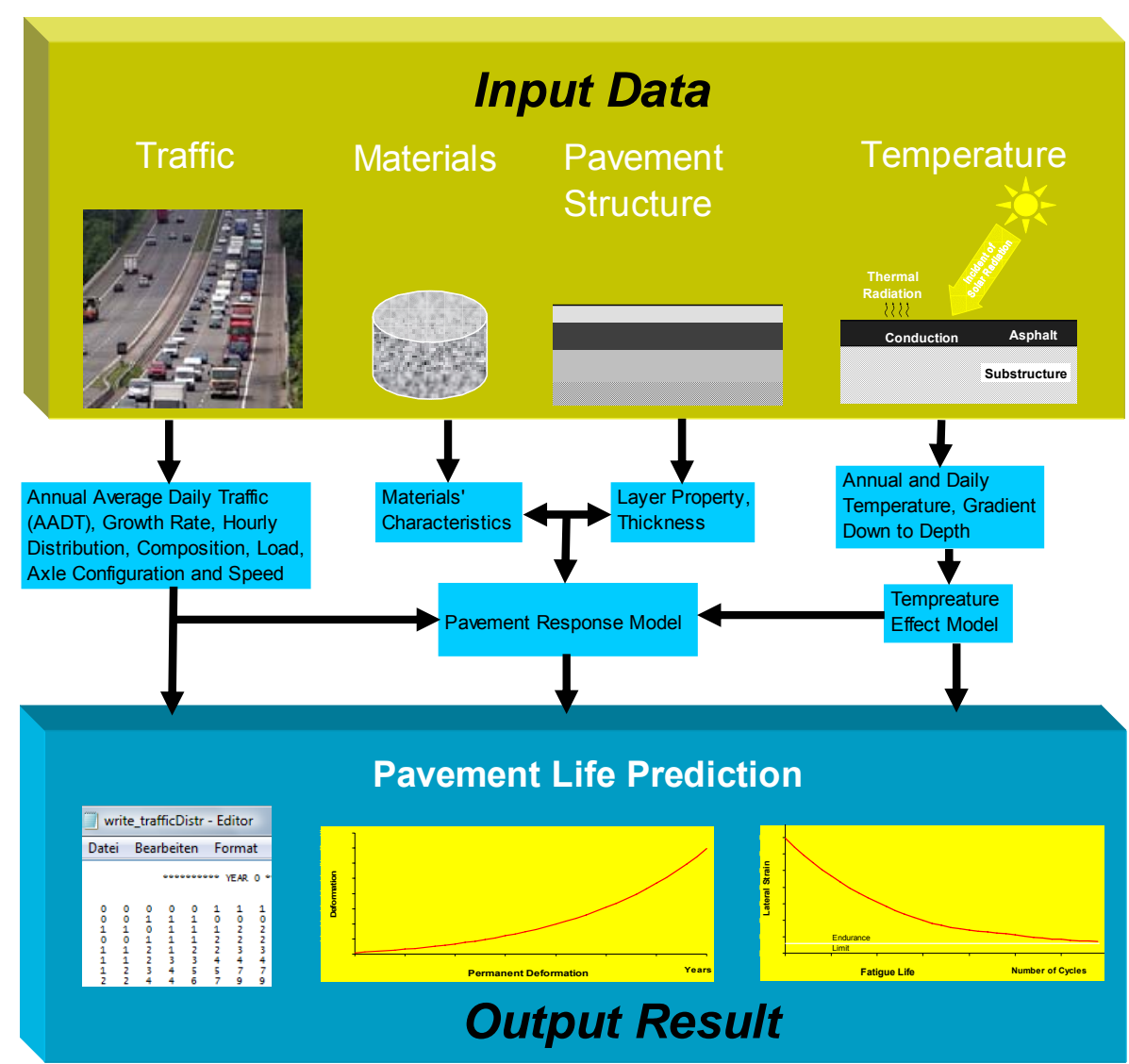

Figure 7.16 Program layout 


\subsubsection{Model Input}

The model has been developed on the bases of external and internal factors assumed to be more significantly affecting the performance of the pavement structure. The most dominant external factors considered in the model have been the traffic characteristics and the temperature change with time. These two inputs in combination with the internal factors (material properties and pavement structural responses) would result in a time dependent deformation output. Here for rutting computation, the loading and unloading periods along with appropriate rut prediction model is necessary. Description of the input variables, their relationships and the model formulations are discussed below.

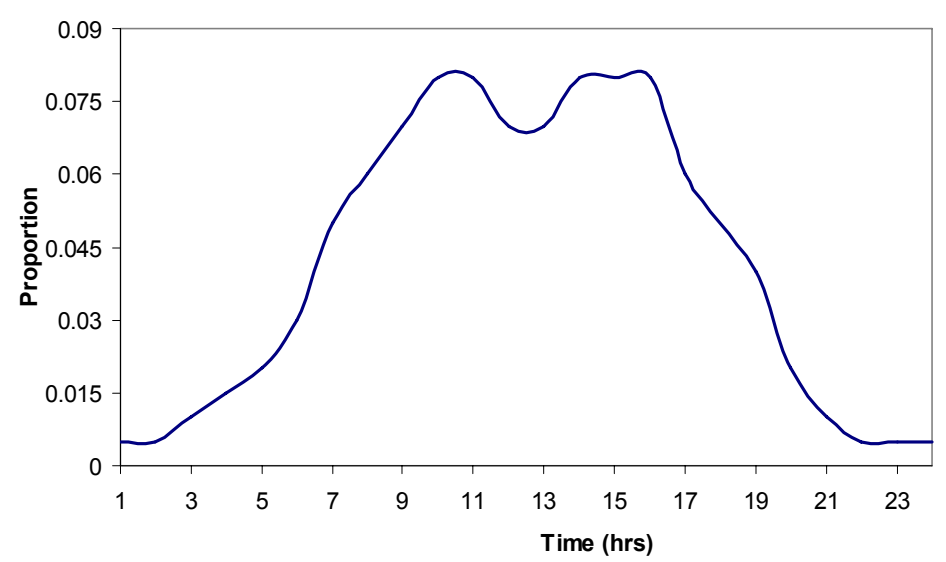

Figure 7.17 Hourly vehicle distribution

\section{Traffic Input}

The type vehicle and magnitude of the load have been segregated according to the category of the vehicle and axle load configuration. It is assumed that the total axle load is equally shared by the available number of wheels mounted on the axle and evenly distributed over a rectangular contact area. The model takes into account the prevailing loading spectrum without converting to the so called ESA loading. Therefore, from known axle load and contact area the contact pressure can easily be computed.

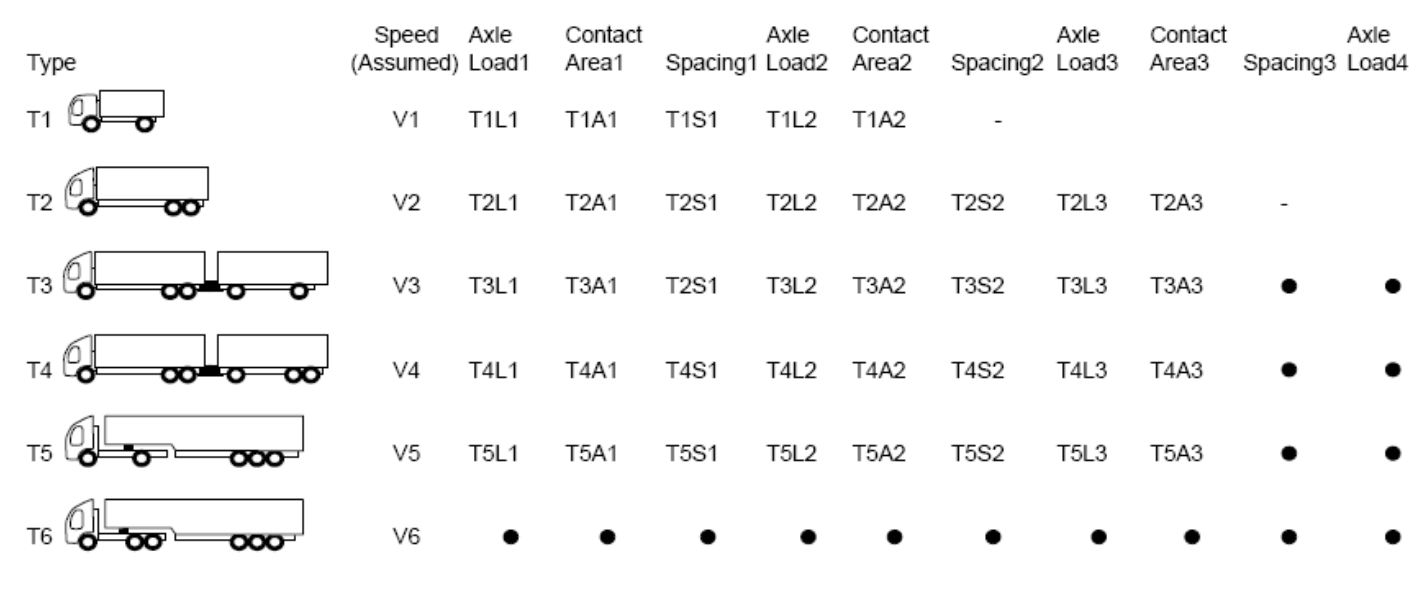

Figure 7.18 Representation of vehicular characteristics in the program

The traffic characteristics have been expressed in terms of the annual growth as discussed in the previous chapter, hourly and daily flow ( $/ \mathrm{hr}, \mathrm{v} /$ day) and corresponding operating characteristics. In some literature [Yang et al. 2009], adjustments are recommended for seasonal variations in traffic, which may be important from traffic engineering point of view. 
Nevertheless, a uniform traffic flow is assumed throughout the season of a year. The annual average daily traffic is estimated for every year from the given base AADT volume and the proposed growth rate and it is assumed that the volume remains constant for that particular year. The hourly volume is an important factor while evaluating the time space (which is part of the unloading times for the model) between consecutive vehicles. It is accordingly adjusted based on the hourly proportion and AADT of a year.

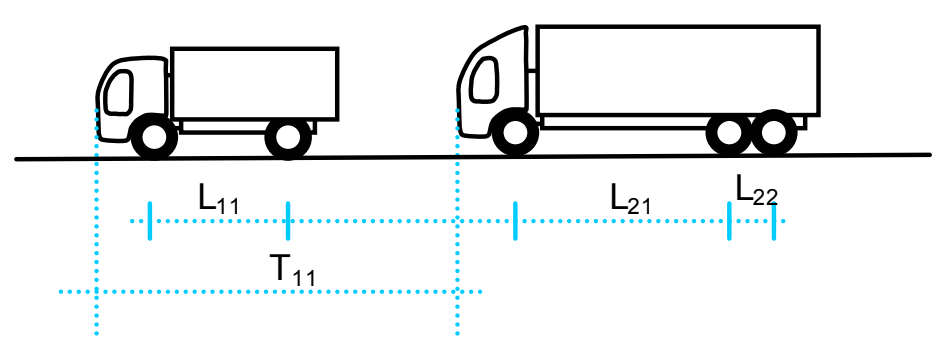

$\mathrm{T}_{11}$-time space between vehicles

$\mathrm{L}_{11}, \mathrm{~L}_{21}, \mathrm{~L}_{22}$-distance between axles

Figure 7.19 Time space between vehicles

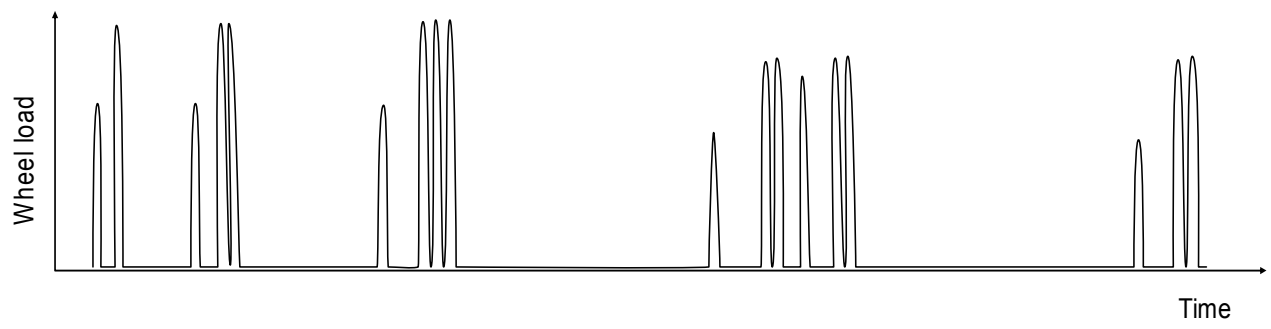

Figure 7.20 Systematic representation of wheel load application with time

For every application of wheel load, there exists unrecoverable strain which ultimately leads to accumulation of permanent deformation. The permanent strain for a single load can be evaluated using equation 4.9. Therefore the total deformation for any number of vehicle loads is evaluated as:

$\Delta P_{\text {Total }}=\sum_{j=1}^{m}\left[\sum_{i=1}^{n}\left[\left(\varepsilon_{\mathrm{i}}^{\mathrm{P}}\right)\left(\Delta Z_{i}\right)\right]\right]$

Where $\Delta P_{\text {Total }}$-total permanent deformation during the service period

$n-$ number of layers

$m$-total number of wheel loads expected during the service life of a pavement

Perhaps, if the total number of types of vehicles and corresponding mounted axles per type are known with proportion of each type, then $m$ can be the total number of axles through out the service life of a pavement.

The other important parameter from the traffic characteristics is the time factor associated with the traffic flow behavior. The time of loading and unloading has been computed from the relationship formulated between speed, time space between vehicles, axle space and wheel contact areas. For simplicity, an equal time space between consecutive vehicles is assumed within each hour according to the hourly traffic flow record (equal to the inverse of the hourly volume). Recent studies indicate that the speed has significant contribution for the deformation of a pavement structure, even though its impact had been totally ignored in many design 
standards, however, it has been indicated [Chen et al. 2004] that the maximum vertical compressive strain at the top of the surface layer corresponding to a travel speed of $20 \mathrm{~km} / \mathrm{h}$ was two times greater than that of $90 \mathrm{~km} / \mathrm{h}$.

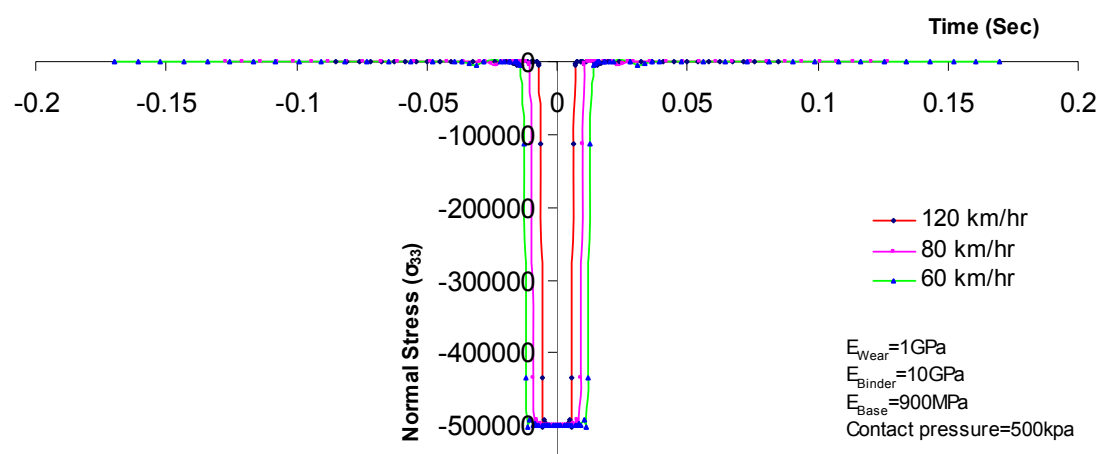

Figure 7.21 Normal stress vs. load duration due to speed variation

The time-stress distribution is related to the traveling speed of the vehicle. The higher the travel speed the narrow band width of the stress distribution, i.e. shorter loading period and vice versa. The frequency of occurrence of the maximum stress at a point also varies with the time span between consecutive vehicles, the traveling speed and the level of service of a road. This can be further related to the density and free flow speed of the traffic stream, which is not discussed here in detail as it is beyond the scope of this work.

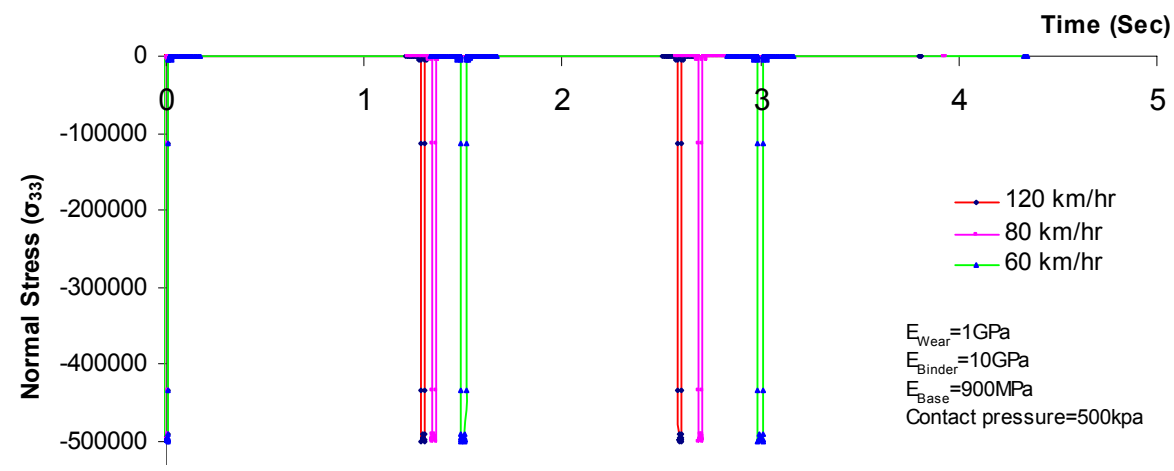

Figure 7.22 Time space variations with respect to traffic speed

Hence the time of loading is taken as the ratio of the contact length (in the direction of travel) to that of the traveling speed for every axle load. Likewise, the unloading time related to each vehicle is computed as the ratio of the distance between axles to that of the corresponding speed of a vehicle. Therefore, the time factors associated to the traffic are the loading period at the contact area and the remaining unloading periods partly with in the time space between successive vehicles and the time span within consecutive axles.

The importance of the traffic wander on the accumulation of pavement permanent deformation is unquestionable. The traffic wander in the transverse direction has constructive effect depending on the width and the number of lane, density of the traffic and other factor. Rate of deformation and pavement life are considerably influenced by the wheel path distribution. When the traffic travels in single track, it accelerates the deformation of a pavement. The position of the wheel load across the width has been assumed to follow statistically normal distribution with the major portion of the traffic travels at the centre of the lane with some portion dispersed across the lane. 


\section{Temperature Input}

The other external factor which has been given a due concern is the temperature variations with time. Accurate prediction of temperature profile in pavements greatly aids for the assessment of pavement deflection and back-calculation of pavement parameters and for estimation of thermalrelated pavement properties. In this work, the temperature has been evaluated applied in two ways, as discussed in chapter 5. On one hand, the effects of the seasonal variations and the gradients within the pavement structure have been assessed in order to evaluate the seasonal variation of rutting in the model. On the other hand, the influence temperature-dependent material parameter and related deformation characteristics have been evaluated.

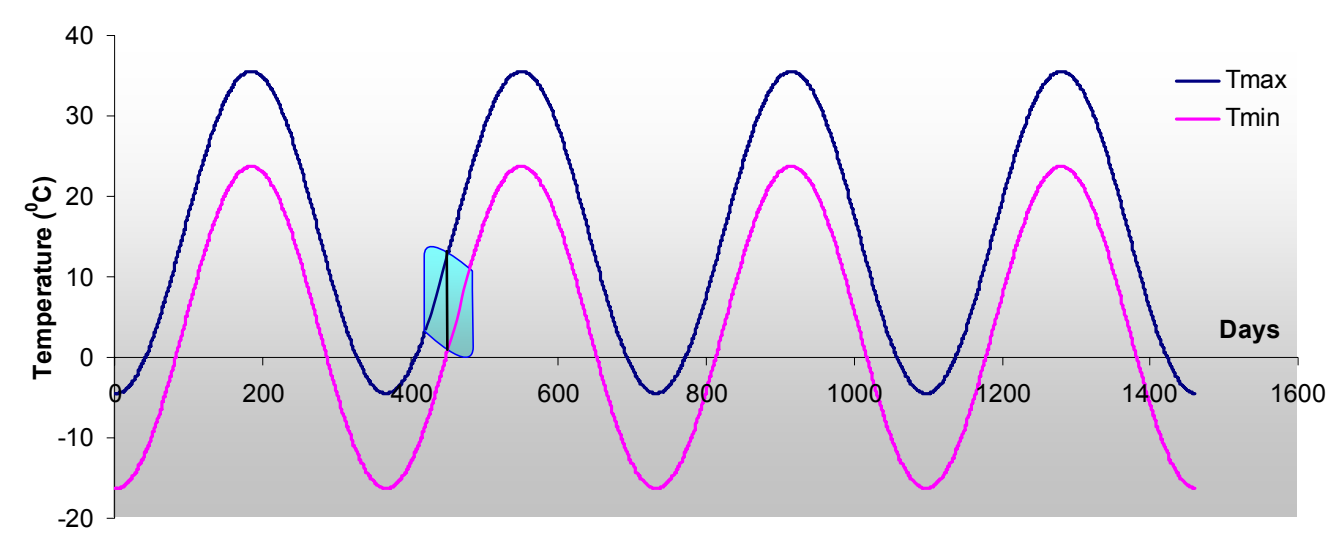

Figure 7.23 Annual temperature fluctuation pattern

As discussed in section 5.1 the annual and daily temperature variations follows some sort of sinusoidal patter. The thermal properties of a pavement depends on several factors such as the intensity of solar radiations, the conductivity behavior, location and many other parameters as discussed in earlier chapter. The annual daily maximum and minimum surface temperature variations are evaluated separately with their corresponding amplitudes and average values. These can also be obtained from recorded metrological data or generated from appropriate temperature models. For this research they have been made to vary for comparison and illustration purpose.

The hourly variations of temperature have been bounded within daily maximum-minimum limit as illustrated below. It is one of the input parameter to assess the temperature gradient and corresponding change in materials' properties within the pavement structure. With this hourly variation, the corresponding temperature can computed at any depth within the pavement structure.

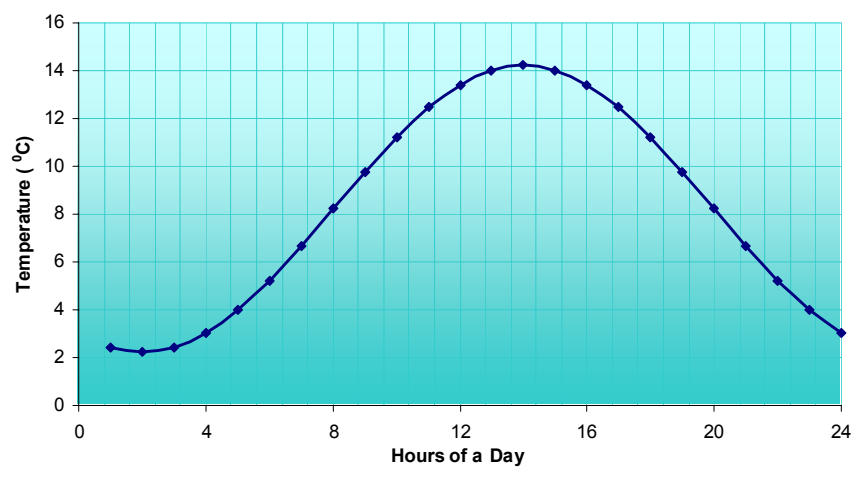

Figure 7.24 Daily temperature variations at selected date 
The temperature fluctuation rather severely affects the material properties and structural responses which ultimately influences the pavement deterioration pattern. In this research, the model adopted by Wellner and Kayser [2008] has been used to evaluate the surface and structural temperature gradients of the pavement. According to section 5.1, the pavement temperature at any depth and given ranges of surface temperature is assumed to follow the pattern as indicated in chapter 5(refer Figure 5.5).

For a given air temperature, the layer temperature is predicted at the required depth based on time of a day, temperature coefficients and other factors. The temperature variation across the pavement depth is used to evaluate the elastic and the viscous parameter of the layers in which the stress-strain state and deformation behaviors are computed. For ABAQUS analysis and to simplify the analysis, the mid-depth temperature gradients are evaluated and corresponding elastic modulus is evaluated from temperature-elastic modulus relationship. With known elastic modulus at a particular temperature, corresponding vertical stress is computed which is later on used in Burgers model.

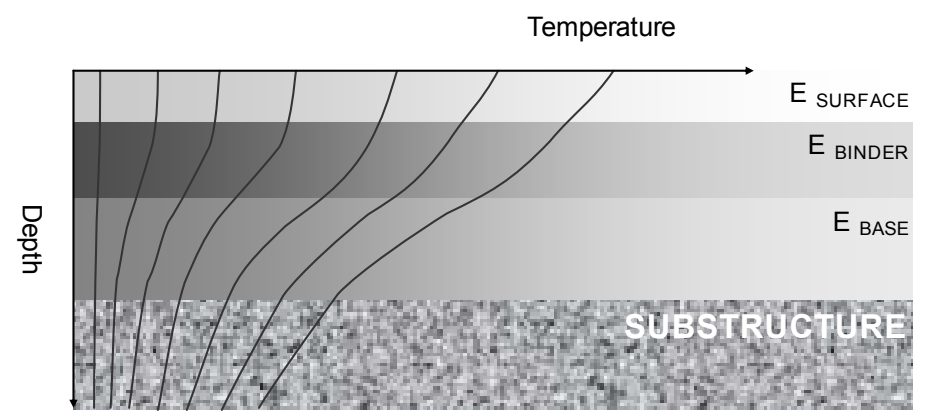

Figure 7.25 Schematic representation of temperature gradient across the depth of a pavement

\section{Material Input}

The internal factors considered in this model have been the materials characteristics and the pavement structural response. The viscoelastic nature of the AC layer brings about the performance pavement highly vulnerable to the magnitude of temperature and load. This time dependent behavior is modeled using the Burgers material model as described in chapter 4 (refer article 4.2.1, Equation 4.25 through 4.28).

The permanent strains of the layers are captured by the strain component formulated at rest period as indicated in Equation 4.26 with known stresses developed at the mid depth and elasticity modulus in each asphaltic layer. The temperature-dependent elasticity moduli of the layers are decomposed into two, i.e., the instantaneous and delayed moduli. The loading and unloading time variables are evaluated from the traffic characteristics as explained above.

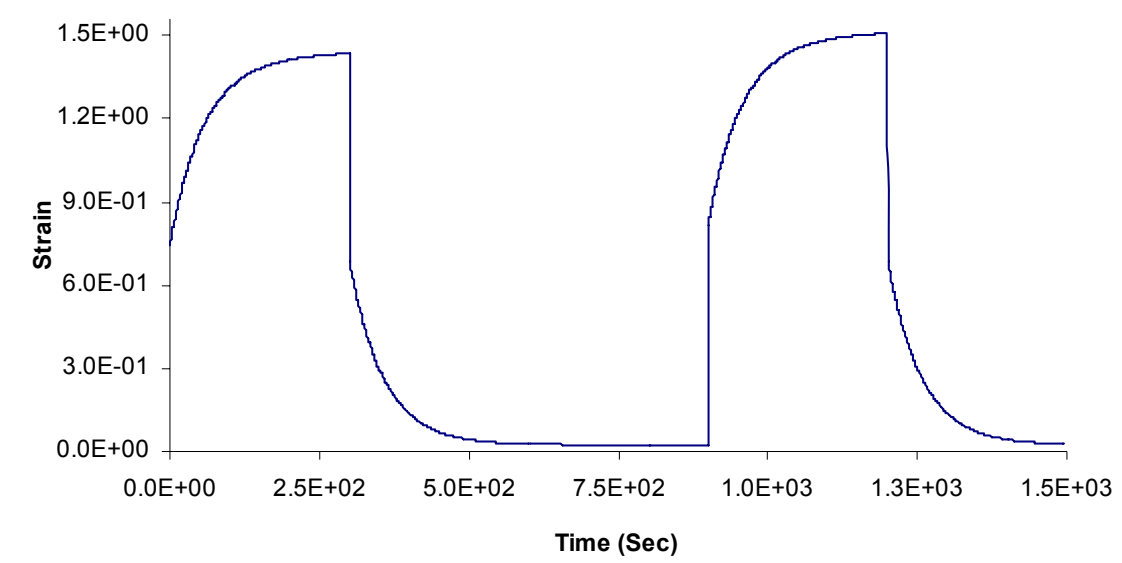

Figure 7.26 Burgers model 
The Burger model requires elastic and viscous components (i.e. $E_{1}, E_{2}, \eta_{1}, \eta_{2}$ ) material input data for rutting analysis. These parameters can be obtained either from laboratory investigation, extracted from master curves or by regression analysis of curve fitting of the data available from literature and laboratory findings. For this work several papers have been looked for to get relevant data for the program input. For illustration purpose the data presented by Uzarowski [2006] is explained as follow.

\begin{tabular}{|c|c|c|c|c|c|c|c|}
\hline \multirow[t]{2}{*}{ Mix } & \multirow{2}{*}{$\begin{array}{c}\text { Frequency } \\
(\mathrm{Hz})\end{array}$} & \multirow{2}{*}{$\begin{array}{l}\text { Time } \\
(\mathrm{sec})\end{array}$} & \multicolumn{5}{|c|}{ Average Modulus ( $\mathrm{kPa})$} \\
\hline & & & $-10.0^{\circ} \mathrm{C}$ & $4.4^{\circ} \mathrm{C}$ & $21.1^{\circ} \mathrm{C}$ & $37.8^{\circ} \mathrm{C}$ & $54.4^{\circ} \mathrm{C}$ \\
\hline HL 3 & $\begin{array}{c}25 \\
10 \\
5 \\
1 \\
0.5 \\
0.1 \\
\end{array}$ & $\begin{array}{c}0.04 \\
0.1 \\
0.2 \\
1 \\
2 \\
10 \\
\end{array}$ & $\begin{array}{l}29,035,597 \\
26,140,927 \\
23,758,520 \\
19,463,937 \\
17,024,643 \\
12,462,463 \\
\end{array}$ & $\begin{array}{c}18,234,080 \\
15,782,363 \\
14,155,470 \\
8,970,193 \\
8,410,651 \\
5,904,908 \\
\end{array}$ & $\begin{array}{l}8,517,013 \\
6,724,647 \\
5,632,195 \\
3,567,388 \\
2,903,347 \\
1,923,555 \\
\end{array}$ & $\begin{array}{c}3,677,663 \\
2,531,608 \\
2,001,509 \\
1,324,485 \\
1,139,086 \\
876,328 \\
\end{array}$ & $\begin{array}{c}1,771,922 \\
1,241,269 \\
1,062,556 \\
789,298 \\
723,110 \\
543,014 \\
\end{array}$ \\
\hline SMA L & $\begin{array}{c}25 \\
10 \\
5 \\
1 \\
0.5 \\
0.1 \\
\end{array}$ & $\begin{array}{c}0.04 \\
0.1 \\
0.2 \\
1 \\
2 \\
10 \\
\end{array}$ & $\begin{array}{l}28,328,933 \\
26,148,687 \\
22,299,133 \\
19,474,150 \\
16,197,897 \\
13,671,253 \\
\end{array}$ & $\begin{array}{r}17,781,917 \\
15,864,307 \\
14,503,093 \\
11,373,943 \\
10,030,364 \\
7,409,310 \\
\end{array}$ & $\begin{array}{l}8,991,958 \\
7,122,266 \\
6,005,836 \\
3,813,784 \\
3,115,649 \\
2,052,178 \\
\end{array}$ & $\begin{array}{c}3,787,753 \\
2,577,009 \\
2,018,145 \\
1,312,803 \\
1,124,041 \\
858,210 \\
\end{array}$ & $\begin{array}{c}1,635,066 \\
1,134,992 \\
939,640 \\
743,254 \\
698,482 \\
660,503 \\
\end{array}$ \\
\hline SMA G & $\begin{array}{c}25 \\
10 \\
5 \\
1 \\
0.5 \\
0.1 \\
\end{array}$ & $\begin{array}{c}0.04 \\
0.1 \\
0.2 \\
1 \\
2 \\
10 \\
\end{array}$ & $\begin{array}{r}21,880,647 \\
19,329,337 \\
17,532,513 \\
13,743,870 \\
12,003,197 \\
8,276,640 \\
\end{array}$ & $\begin{array}{c}14,303,734 \\
12,316,603 \\
10,777,247 \\
7,607,238 \\
6,335,759 \\
4,028,829 \\
\end{array}$ & $\begin{array}{l}5,971,156 \\
4,511,806 \\
3,689,282 \\
2,286,621 \\
1,868,067 \\
1,231,840 \\
\end{array}$ & $\begin{array}{c}2,475,618 \\
1,719,027 \\
1,406,425 \\
1,003,376 \\
902,241 \\
749,947 \\
\end{array}$ & $\begin{array}{c}1,401,389 \\
1,043,258 \\
903,193 \\
733,605 \\
683,879 \\
582,696 \\
\end{array}$ \\
\hline SP $19 \mathrm{D}$ & $\begin{array}{c}25 \\
10 \\
5 \\
1 \\
0.5 \\
0.1 \\
\end{array}$ & $\begin{array}{c}0.04 \\
0.1 \\
0.2 \\
1 \\
2 \\
10 \\
\end{array}$ & $\begin{array}{l}30,403,193 \\
27,300,243 \\
25,117,033 \\
21,139,430 \\
18,947,950 \\
14,549,327 \\
\end{array}$ & $\begin{array}{c}21,183,310 \\
18,869,130 \\
17,367,207 \\
14,062,450 \\
12,641,303 \\
9,637,153 \\
\end{array}$ & $\begin{array}{c}11,078,390 \\
9,208,961 \\
8,078,782 \\
5,656,008 \\
4,764,570 \\
3,307,524 \\
\end{array}$ & $\begin{array}{l}5,898,574 \\
4,534,139 \\
3,780,130 \\
2,508,557 \\
2,127,961 \\
1,547,471 \\
\end{array}$ & $\begin{array}{l}2,975,443 \\
2,165,085 \\
1,806,861 \\
1,349,179 \\
1,224,906 \\
1,036,457 \\
\end{array}$ \\
\hline SP $19 E$ & $\begin{array}{c}25 \\
10 \\
5 \\
1 \\
0.5 \\
0.1\end{array}$ & $\begin{array}{c}0.04 \\
0.1 \\
0.2 \\
1 \\
2 \\
10 \\
\end{array}$ & $\begin{array}{l}27,417,293 \\
24,465,313 \\
22,305,090 \\
18,038,760 \\
15,865,667 \\
11,332,473\end{array}$ & $\begin{array}{c}19,913,503 \\
17,316,047 \\
15,563,347 \\
11,729,560 \\
10,040,225 \\
6,810,045\end{array}$ & $\begin{array}{c}10,709,234 \\
8,545,018 \\
7,151,440 \\
4,506,336 \\
3,606,568 \\
2,272,055\end{array}$ & $\begin{array}{l}4,847,736 \\
3,457,608 \\
2,817,195 \\
1,957,403 \\
1,725,655 \\
1,370,845\end{array}$ & $\begin{array}{l}2,234,002 \\
1,695,693 \\
1,484,138 \\
1,242,169 \\
1,175,920 \\
1,088,088\end{array}$ \\
\hline
\end{tabular}

Table 7.1 Summary of dynamic modulus investigated by Uzarowski [Uzarowski 2006]

The data variables and time ranges are imported into the MATLAB Curve Fitting Tool for a given temperature. The data sets are fitted to a curve with an exponential of a second degree order. Then the data, model fit, coefficients with confidence bound and residuals are obtained as shown in Figure 7.27. The procedure is further continued to obtain the required material parameter at different temperatures.
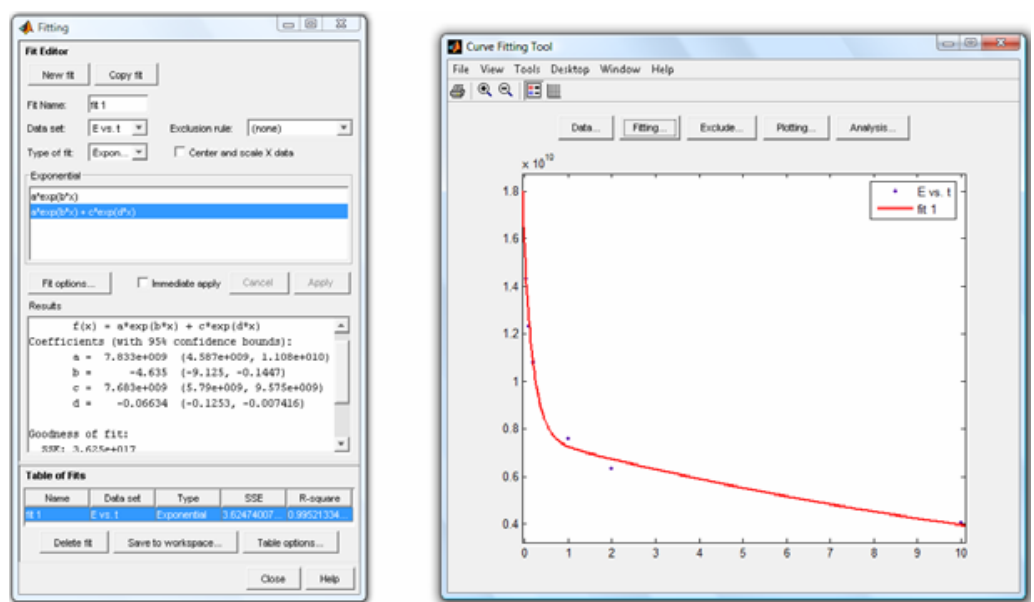

Figure 7.27 MATLAB regression analysis

The curve fit is obtained in the form of: 


$$
f(x)=a e^{b x}+c e^{d x}
$$

The independent variable $x$ and function $f(x)$ are representing time variable $t$ and timedependent elastic modulus $E(t)$, respectively. The coefficients $a$ and $b$ are the instantaneous and delayed elastic modulus components, respectively. The viscous components of the materials are evaluated from the coefficients $c$ and $d$. Rewriting equation 4.28 gain,

$$
E(t)=E_{1} e^{\left(\frac{-E_{1} t}{\eta_{1}}\right)}+E_{2} e^{\left(\frac{-E_{2} t}{\eta_{2}}\right)}
$$

With $\quad a=E_{1}, \quad b=E_{2}, \quad c=\frac{-E_{1}}{\eta_{1}}, \quad d=-\frac{-E_{2}}{\eta_{2}}$

The regression analysis typically carried out on SMA G mix at $4.4{ }^{\circ} \mathrm{C}$ is given in the Table 7.2 below. Therefore the time dependent elasticity is expressed in the form as given in equation 7.3:

$$
E(t)=7.683 \times 10^{9} e^{-0.06634 t}+7.833 \times 10^{9} e^{-4.635 t}
$$

Where $E(t)$ - time dependent modulus (pa)

$$
t \text { - time (s) }
$$

The table below shows summary of the regression analysis result and Burgers materials parameter which are extracted form the regression.

\begin{tabular}{lllllll}
\hline Temp $\left({ }^{0} \mathrm{C}\right)$ & $\mathrm{a}\left(\mathrm{E}_{1}\right)[\mathrm{pa}]$ & $\mathrm{b}$ & $\mathrm{c}\left(\mathrm{E}_{2}\right)[\mathrm{pa}]$ & $\mathrm{d}$ & $\eta_{1}[\mathrm{pa} . \mathrm{s}]$ & $\eta_{2}$ [pa.s] \\
\hline-10 & $1.94 \mathrm{E}+10$ & -0.05319 & $9.46 \mathrm{E}+09$ & -4.855 & $3.65 \mathrm{E}+11$ & $1.95 \mathrm{E}+09$ \\
4.4 & $7.68 \mathrm{E}+09$ & -0.06630 & $7.83 \mathrm{E}+09$ & -4.635 & $1.16 \mathrm{E}+11$ & $1.69 \mathrm{E}+09$ \\
21.1 & $2.32 \mathrm{E}+09$ & -0.06618 & $4.64 \mathrm{E}+09$ & -6.536 & $3.51 \mathrm{E}+10$ & $7.09 \mathrm{E}+08$ \\
37.8 & $1.02 \mathrm{E}+09$ & -0.03255 & $2.10 \mathrm{E}+09$ & -9.759 & $3.14 \mathrm{E}+10$ & $2.15 \mathrm{E}+08$ \\
54.4 & $7.48 \mathrm{E}+08$ & -0.02593 & $9.84 \mathrm{E}+08$ & -10.73 & $2.89 \mathrm{E}+10$ & $9.17 \mathrm{E}+07$ \\
\hline
\end{tabular}

Table 7.2 Summary of regression results for SMA G mix
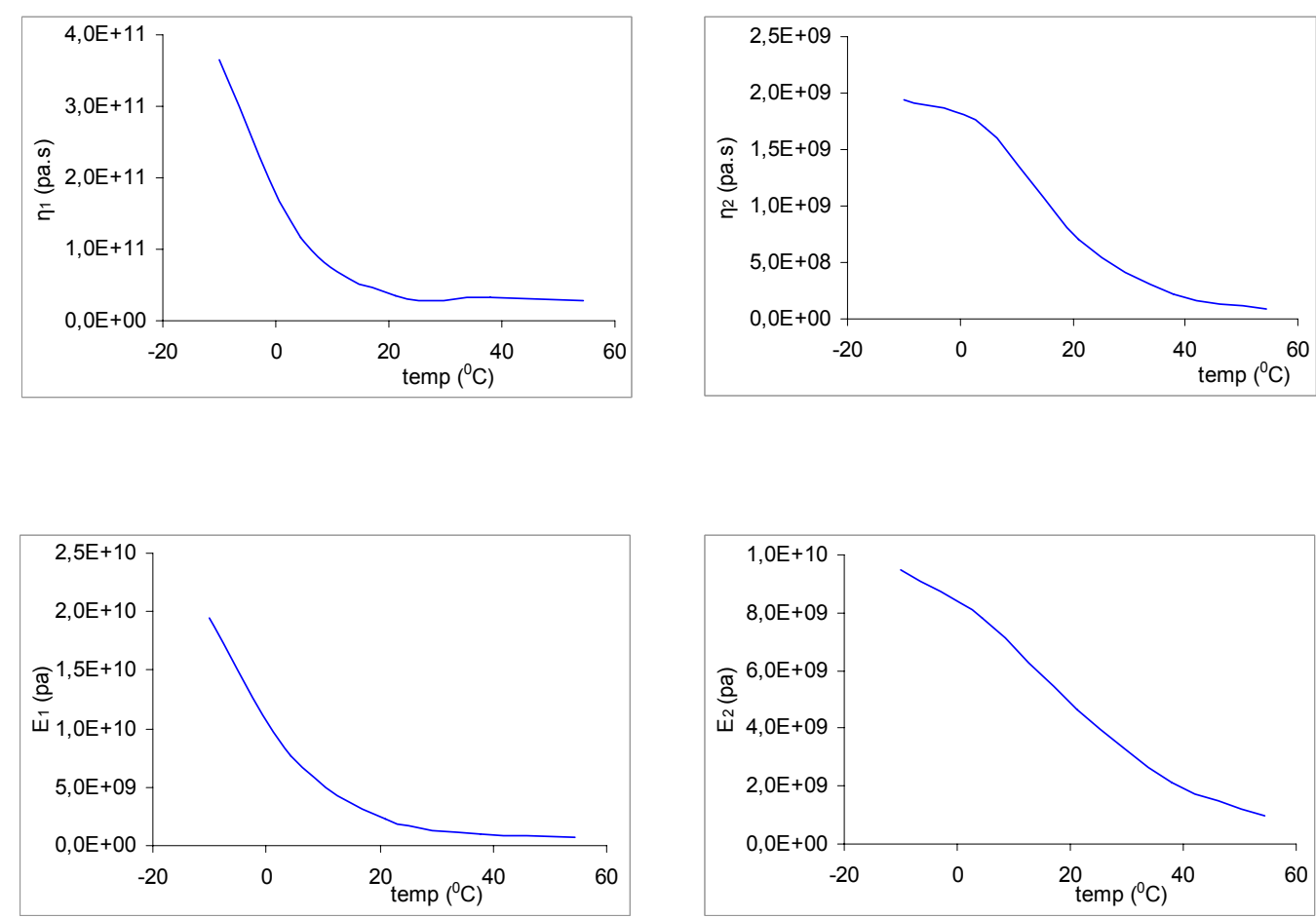

Figure 7.28 Burgers parameters variations with respect to temperature for SMA G mix 


\section{Structural Input}

The geometry of the structure and the layer characteristics are required variables to compute the deformation progress. Thicknesses of each layer are given in a file. The temperature dependent elastic modulus of each layer and corresponding mid depth stresses are the structural input variables of the models. For a particular time of a day, the air temperature and its gradient across the depth are evaluated according to (Equation 5.14). Therefore from the temperatureelasticity modulus relationship, the layer elasticity modulus at a particular temperature and time can be determined. At high temperature, the elasticity modulus of asphalt concrete drops down which leads a rapid rutting progress. Such failures are predominantly revealed at the top surface due high compressive stresses in the AC layers and relatively higher surface temperatures.

It is this elastic modulus, at a particular time and temperature, which is used to evaluate the stress component and later applied in the Burgers model while evaluating the permanent strain.

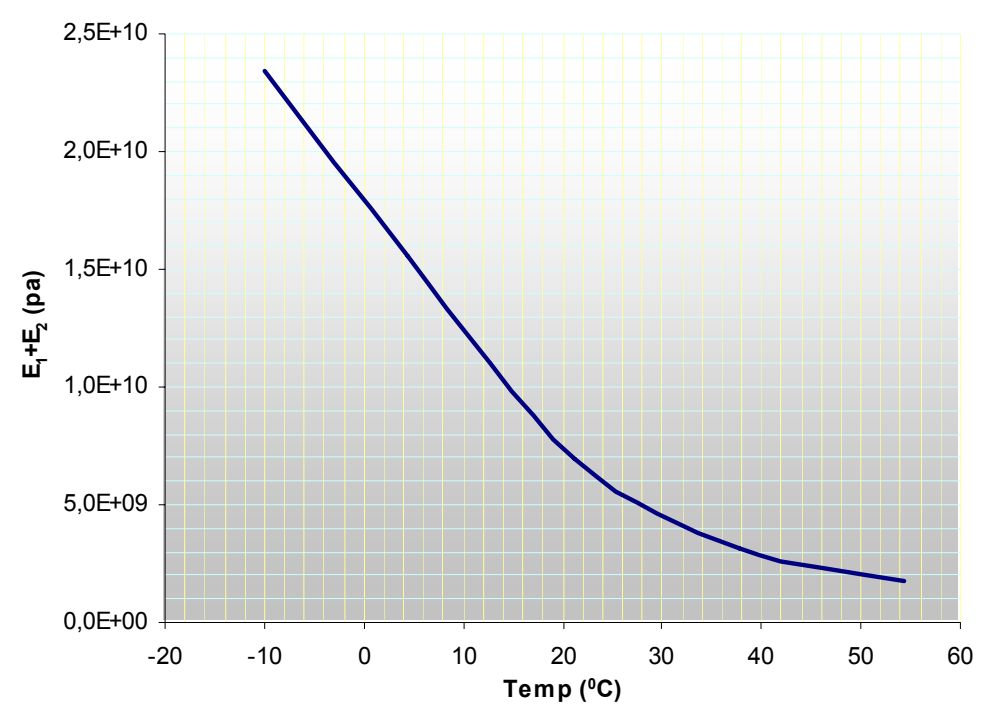

Figure 7.29 Elastic modulus change with respect to temperature

The pavement response has been evaluated using ABAQUS for various load, material properties and structural geometries. The stresses developed at the mid depth of the layers are then interpolated from the analysis results. The time dependent temperature effect has been superimposed with the various vehicular load and flow characteristics in order to provide an input for the Burger models that ultimately predicts the accumulation of deformation for the presumed service time. For a given specific time, pavement temperature is evaluated to determine the structural response at that particular time. 


\subsubsection{Program Structure}

The program, which is written with C programming language, has several subroutines intended for different tasks.
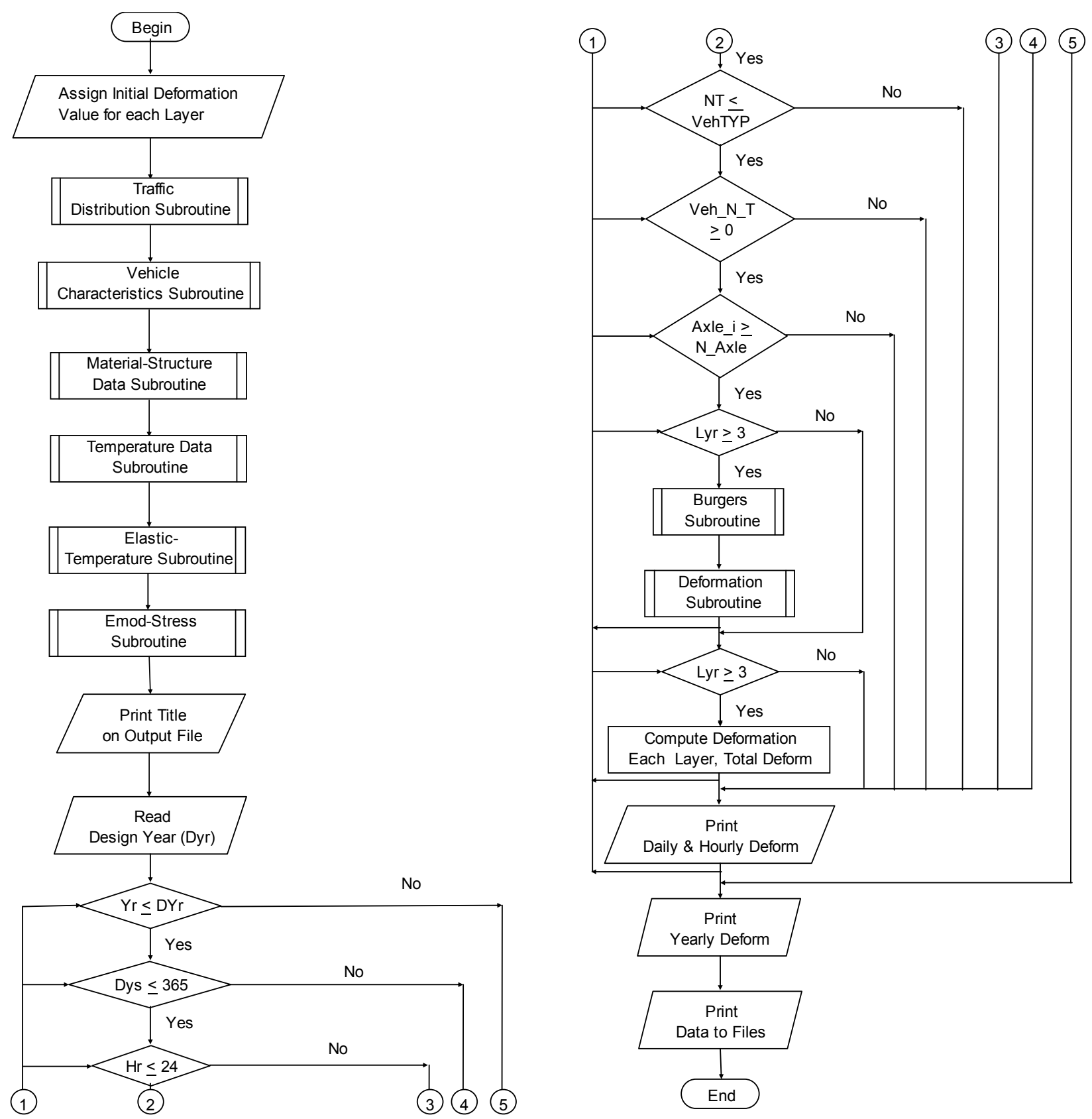

Figure 7.30 Flow chart for the main program

Within the given time frame, the vehicular characteristics, temperature and pavement responses are evaluated. As mentioned above, the program computes time increment on the bases of the hourly traffic volume and vehicular speed. Within the interval of the arrival of vehicles, the time variables of loading and unloading times determined each of which are applied in the Burgers equation.

The program has subroutines to read input and write output. Traffic Distribution subroutines read the traffic input of AADT, annual growth rate, number of traffic type, hourly traffic distribution and proportion of traffic from a text file. The program also returns the hourly flow of traffic for each vehicle type and sum up total flow in each hour. Further more it computes the 
average daily traffic for each year and writes on a file. These variables are used to classify the vehicles according to their type and determine their arrival times. In addition to assigning a time factor for each load, the subroutine takes care of loss of data due to rounding off.

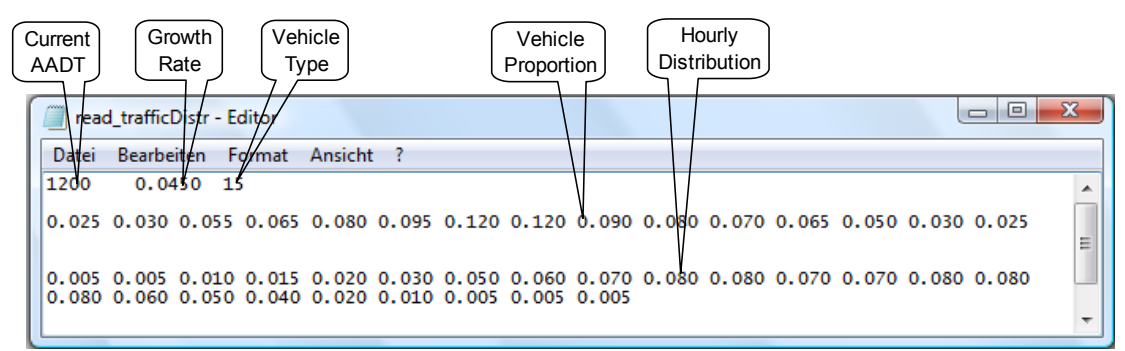

Figure 7.31 Traffic distribution input file

Vehicle Characteristics Program reads the number of traffic type, speed of each vehicle type, axle load and corresponding contact area as well as spacing between axles from a file. Loading characteristics given in a file are used to evaluate the contact pressure on the model. The stress developed with in the pavement section is assumed to be proportional to applied contact pressure. The program returns the loading and unloading times that serves as an input for burgers models. Similarly the temperature, materials and structural data are manipulated with corresponding subroutines. Depending on the temperature, the layer elastic modulus and the stress responses from Abaqus analysis are interpolated and evaluated.

Determination of the permanent strain is taken care of by the Burger subroutine, which evaluates strains in each asphaltic layer and computes deformation with respect to corresponding thicknesses. The total deformation is summed up for each load application as explained in equation 7.1.

To summarize, the objective of the model development is to address the rutting problem within the asphaltic layer. In most design analysis, rutting is associated with the vertical strains experienced by the subgrade layer. The contribution of he the AC layers have been neglected and rather these layers have been analyzed with respect to thermal and load associated fatigue damages. Of course, the assumption is worthy from the perspective of thin asphaltic layers. However, current developments have been indicating that thicker AC pavements suffer due to rutting arising from the superstructure. It has also been supported that thicker AC layers resting on strong and sound foundation layer brought little or no rutting problem on the substructure, i.e., the permanent deformation is confined within the super structure. Due to the fact that AC layers are viscoelastic in nature, subjected to high stress-strain state (due to high traffic volume and axle load) combined with high thermal state on the upper structure make the super structure vulnerable to rutting.

In the model, AC layers have been treated as viscoelastic materials resting on the strong substructure (subbase and subgrade layers). The model has four components; the traffic, temperature, structural and materials components. The traffic load and thermal response the structural and material components are treated simultaneously. At a particular time the surrounding of a pavement structure experiences/or exist/ under specific thermal condition. The thermal condition is associated with the material properties. When a load is applied, the permanent deformation is computed at these material states. The viscoelastic properties of AC layers are sensitive to temperature of the surrounding. In order to evaluate such properties and to the associated permanent deformation, the surrounding ambient temperature is characterized by the sinusoidal curves which varies with respect to hours of a day and days of the year. Further more, the thermal condition of the superstructure is evaluated and the layer properties are determined. 
One of the most important parameter is the time variable associated with loading and unloading. It has been assumed that the hourly volume is evenly spaced with respect to time, hence the unloading time is equivalent to the time between the consecutive vehicles (or same as the inverse of the hourly volume, on the other hand, the loading time is take as the ratio of the contact length to the speed of the vehicle. For this particular case, the timing has been set to start at the beginning of a year (but it can be shifted to start at any convenient time).

The permanent strain for any layer is determined from burgers equation from known applied stress (obtained from FE analysis), viscoelastic layer properties and time variables (obtained from traffic characteristics). The permanent deformation contributed by each vehicle has been summed up to obtain the cumulative rutting throughout the service period.

\subsubsection{Model Output}

Several outputs are written in files. Among which, daily and annual accumulation of deformation are the most required outputs. The model output also includes the hourly traffic volume throughout the service life as well as hourly and daily temperature variations for further manipulation of the output results.

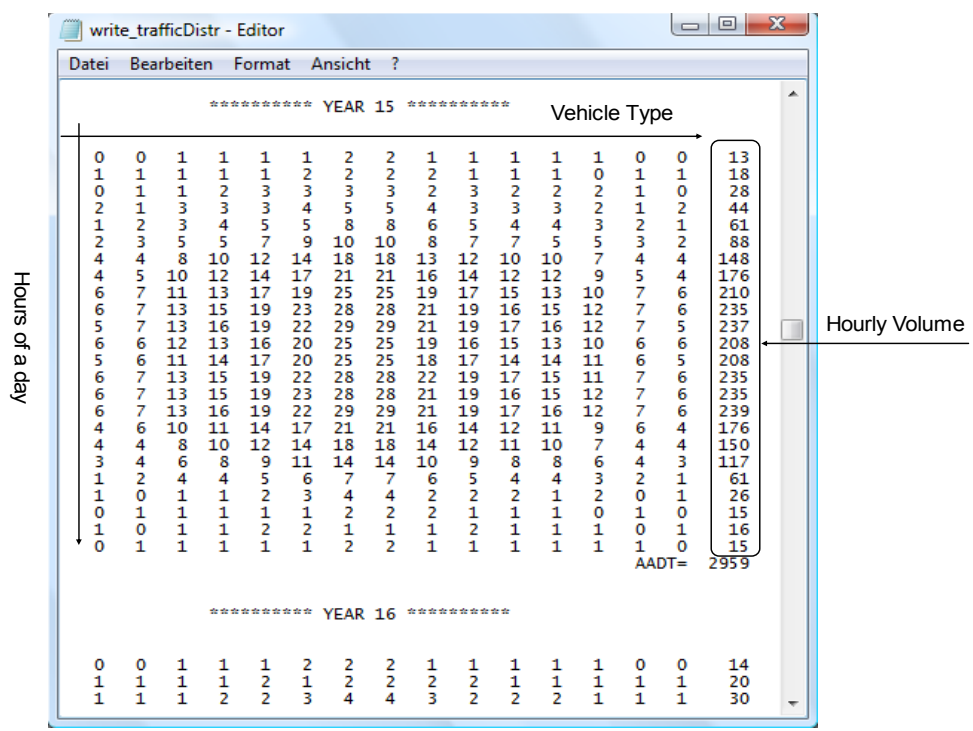

Figure 7.32 Model traffic distribution output

Various deformation behaviors are obtained by varying input parameters. As it is indicated in subsequent charts, an attempt has been made to evaluate the model deformation output with different traffic characteristics, surface temperature and material parameters as well as structural geometries.

The initial traffic volume (expressed in terms of commercial vehicles per day, cv/d), composition and loading characteristics as well as the operating speed of heavy vehicles dominantly affect the rutting behavior. The analyses made to evaluate the influence of the traffic characteristics over the deformation behavior are presented as follow. The traffic characteristics are individually treated in the model. As discussed earlier, the applied wheel load for every vehicle is taken into account and associated with the stress presumably produced with in the asphaltic layers. In the computation, the input variables of growth rate, volume, distribution and other variables are taken into account. The wheel load and corresponding axle configuration are taken from standard vehicle as given in the Appendix A-2. The hourly traffic volume is assumed to follow a pattern as illustrated in Figure 7. 17 in which the highest hourly volume occruring at mid-day. It is applied to evaluate the time-spacing of consecutive vehicles and the wheel load of each for certain sequences of time. 
For illustration purpose, different traffic growth rates are assumed and corresponding characteristics are given in Figure 7.36. The following table shows arbitrary selected initial traffic volume (with growth rate of 4.5\%) and corresponding forecasted volume at different time period. For comparison purpose the equivalent single wheel load (ESAL) is also indicated based on factors recommended on Table A-1 (Appendix A).

\begin{tabular}{|c|c|c|c|c|c|c|c|c|c|c|}
\hline \multirow[b]{3}{*}{$\begin{array}{l}\text { Desig } \\
\text { nation }\end{array}$} & \multirow[b]{3}{*}{$\begin{array}{l}\text { Initial } \\
\text { Traffic }\end{array}$} & & \multicolumn{8}{|c|}{ Total traffic volume forecast at different time period } \\
\hline & & & \multicolumn{2}{|c|}{10 years } & \multicolumn{2}{|c|}{20 years } & \multicolumn{2}{|c|}{30 years } & \multicolumn{2}{|c|}{40 years } \\
\hline & & \multirow{10}{*}{ 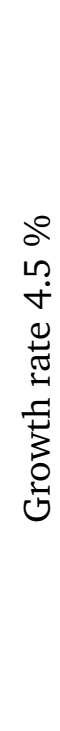 } & $\mathrm{ADT}_{10}$ & $\begin{array}{l}\mathrm{ESAL}_{80 \mathrm{kN}} \\
\text { (Million) }\end{array}$ & $\mathrm{ADT}_{20}$ & $\begin{array}{l}\text { ESAL }_{80 \mathrm{kN}} \\
\text { (Million) }\end{array}$ & $\mathrm{ADT}_{30}$ & $\begin{array}{l}\mathrm{ESAL}_{80 \mathrm{kN}} \\
\text { (Million) }\end{array}$ & $\mathrm{ADT}_{40}$ & $\begin{array}{l}\text { ESAL }_{80 k N} \\
\text { (Million) }\end{array}$ \\
\hline $\mathrm{T} 1$ & 100 & & 155 & 0.40 & 241 & 1.02 & 375 & 1.99 & 582 & 3.49 \\
\hline $\mathrm{T} 2$ & 200 & & 311 & 0.80 & 482 & 2.04 & 749 & 3.97 & 1163 & 6.97 \\
\hline T3 & 300 & & 466 & 1.20 & 466 & 3.07 & 1124 & 5.96 & 1745 & 10.46 \\
\hline $\mathrm{T} 4$ & 500 & & 776 & 2.00 & 1206 & 5.11 & 1873 & 9.94 & 2908 & 17.43 \\
\hline $\mathrm{T} 5$ & 800 & & 1242 & 3.20 & 1929 & 8.18 & 2996 & 15.90 & 4653 & 27.89 \\
\hline $\mathrm{T} 6$ & 1000 & & 1553 & 4.00 & 2412 & 10.22 & 3745 & 19.87 & 5816 & 34.87 \\
\hline $\mathrm{T} 7$ & 1200 & & 1864 & 4.80 & 2894 & 12.26 & 4494 & 23.85 & 6980 & 41.84 \\
\hline $\mathrm{T} 8$ & 1500 & & 2329 & 6.00 & 3618 & 15.33 & 5618 & 29.81 & 8725 & 52.30 \\
\hline T9 & 2000 & & 3106 & 8.00 & 4823 & 20.44 & 7491 & 39.74 & 11633 & 69.73 \\
\hline
\end{tabular}

Table 7.3 Traffic characteristics and forecast at different period time

It is worthy to set some sort of boundary limit with reference to material parameter and traffic volume. Some material input parameters are partly collected from literatures and have been used to delineate especially of lower limits, since beyond which the material parameters are either not worthy due to inadequacy in order to sustain the applied load or may bear unnecessary exaggerated result which may mislead. The materials parameters investigated by Szydlo and Mackiewicz [2005] at $45{ }^{\circ} \mathrm{C}$ are used, as there is fairly enough to assume the analysis result based on these material properties are on the safer side. The material parameters used for subsequent charts are taken from Table B1 through B3. Material parameters B2a and B2b represent the lower and upper limit of the material properties given in Table B2. With this material parameter, the vertical stress with in the model has been evaluated using ABAQUS. The analysis result with a structural thickness of 50,100 and $100 \mathrm{~mm}$ for wearing, binder and base course layer, respectively, is shown in the Figure 7.33 below. The stress indicated in the figure is the maximum vertical stress along the vertical selected path directly below the loaded/contact/ area. 


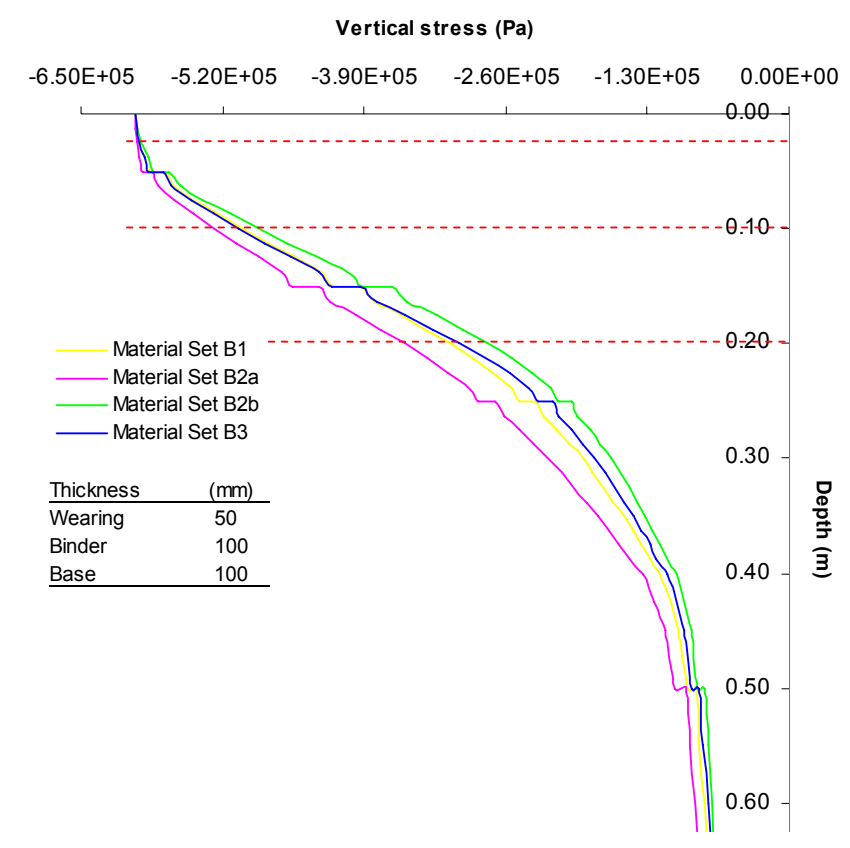

Figure 7.33 Vertical stress

To set a lower limit for material property and traffic volume, the following analysis is carried out on two material parameters with different traffic volume. The material parameters of B1 and B2a (lower limit in Table B2) are selected (given in Appendix B). It has been found that the material parameters have a pronounced effect on rut formation with lower values obviously resulting in higher permanent deformation even for low traffic volume. The pavement structure with materials properties at lower range (B2a) performs satisfactorily for low traffic volume as indicated in Figure 7.34 (a) below. The deformation value of $25 \mathrm{~mm}$ is expected for a traffic volume of 3.90 million single axle (msa) load. The design life depends on the amount of the initial traffic, i.e. the pavement probably serves for about 30- or more than 40- years when the initial traffic is 200 or $100 \mathrm{cv} / \mathrm{d}$, respectively.

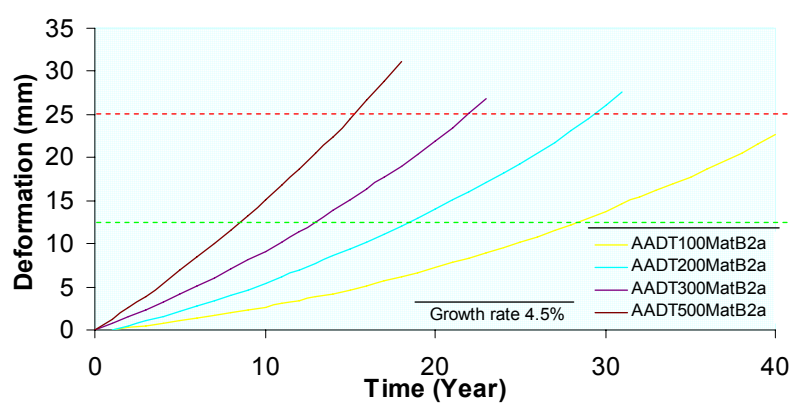

a. Deformation with material characteristics B2a

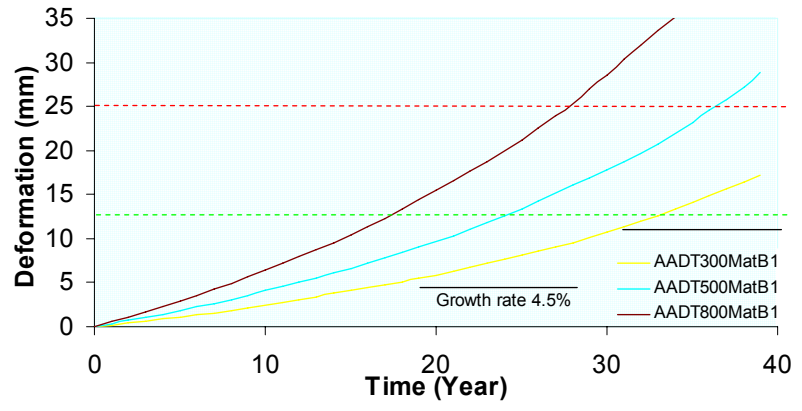

b. Deformation with material characteristics B1

Figure 7.34 Deformation characteristics with respect to traffic volume and materials characteristics

On the other hand, the deformation characteristics illustrated in Figure 7-34 (b) shows that material parameters described as B1 performs well under intermediate traffic volume (as the parameters in material B1 have relatively better value than B2a)and can serve for about 14.0 msa loading within the limits of rutting. The service life depends, likewise, on the initial traffic flow.

Taking into account higher range of the material parameters (B2b) from Table B2, the following results are obtained for different initial traffic volume (1000, 1500 and $2000 \mathrm{cv} / \mathrm{d}), 4.5 \%$ traffic growth rate. The deformation property indicates that the pavement structure with B2b material 
properties would serve relatively longer period of time (in the range of 30 - 35 years) when the initial traffic is around or below $1000 \mathrm{cv} / \mathrm{d}$. On the other hand, with the same material behavior, the pavement might fail at early service life when the initial traffic is very high (in case of 2000 $\mathrm{CV}$ /day initial traffic volume) and a permanent deformation of $25 \mathrm{~mm}$ may occur at the service life of 25 years or before. With respect to their corresponding ESAL, the pavement shows almost the same failure value for the three traffic volume roughly around the same ESAL (about 30 msa) regardless of the initial traffic volume but with significant variation in service life. It is clear that the deformation characteristics and the service life of a pavement are highly dependent on the magnitude of the initial traffic volume. Pavement structures subjected to such high traffic volume are required to be designed and constructed with material of best qualities in order to achieve the maximum service life.

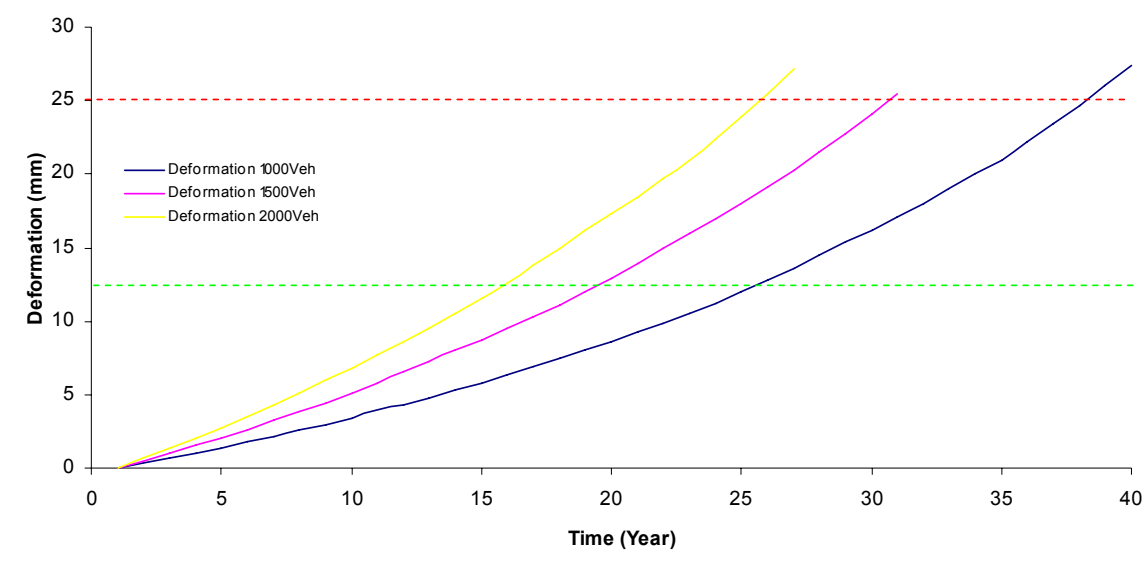

Figure 7.35 Deformation characteristics with respect to traffic volume and materials characteristics

From the above analyses, the deformations are mainly dependent on the viscous component of the model as well as the intensity of the traffic. It is obvious that the higher the viscous component of the material, the better is the serviceability of the road without under going excessive deformation. As can be seen above a model structure with material parameter described in B2 sustains higher traffic volume and performs better than the rest due to higher immediate viscosity parameter $\eta_{1}$.

The sensitivity of deformation characteristics with respect to growth rate has been a great concern while predicting the pavement life. The actual growth rate of traffic itself depends on many factors; however, its influence on rutting and associated life span has been given more concern in subsequent charts. The figures below show the influence of the growth rate (ranging from $2.5 \%-7.5 \%$ ) on traffic volume and permanent deformation. With the same initial traffic volume $(1500 \mathrm{cv} / \mathrm{d})$ and material parameter $\mathrm{B} 2 \mathrm{~b}$, it expected that the rutting accelerates with high traffic growth rate.

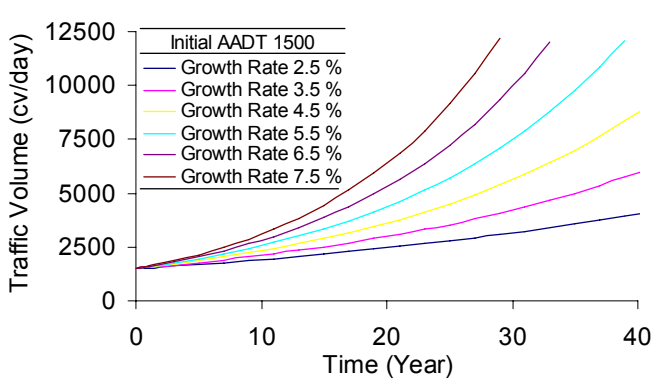

a. Traffic forecast for different growth rate

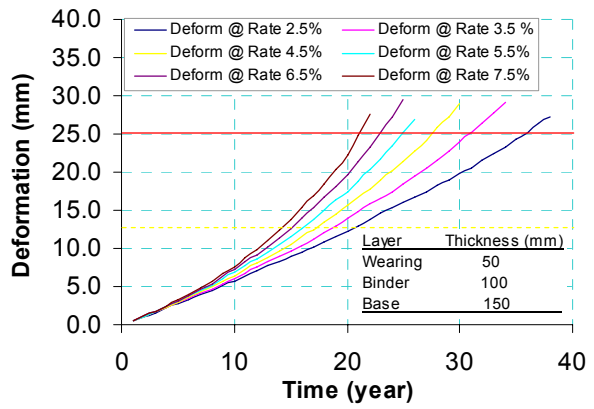

b. Effect of growth rate on permanent deformation

Figure 7.36 Trend of AADT with rates of growth and corresponding permanent deformation 
At the end of the service year, the number of vehicles obviously increases very rapidly (depending on the rate of traffic growth). Besides the higher number of load applications, such circumstances demand relatively longer loading time (due to traffic congestion and lower operating speed) and/or shorter unloading time. This time factor can be directly associated with the rate of rutting. As can be seen from Figure 7.36 (b), the slope of the deformation curve sharply increases at later time of the service year, i.e., the rate of rutting is higher due to higher number of vehicles' load applications (due to the exponential growth of the traffic). Dramatic increases in the number of vehicles have resulted in severe rutting. The deformation progress has found highly sensitive to the traffic growth rate as shown in the figure above. Keeping material and structural properties the same, the deformation curve has shown a rapid increment at later years with $7.5 \%$ growth rate and it accelerates the permanent deformation the structure. This suggests that design standards are required to thoroughly choose applicable rate to correctly address the associated rutting problems, to estimate the required structural elements with a reasonable degree of accuracy as well as to make a rational decision on design related matters. Therefore, it is important to accurately determine the annual growth rate of the traffic to minimize over- or under-estimation of the pavement structure. Failure in addressing such factors reasonably may lead inappropriate design. In addition, providing better quality of AC materials is unquestionable when the traffic volume and/or growth rate are presumed to be high.

The above conservative analyses are rather based on a single temperature $\left(45^{\circ} \mathrm{C}\right)$ where the pavement is not always exposed. Material parameters values at this temperature exist at their intermediate range and the load-associated deformation is some how greater than the one computed with normal temperatures. The next step is to analyze the influence of the temperature fluctuation, material property and the role of structural geometry on permanent deformation of a pavement.

The temperature variables can be analyzed from geographical and metrological records. The annual temperature formulation requires the maximum and minimum variations. For this work, these variables are formulated with assumed magnitudes of the average and amplitudes of the temperature as well as the period of the temperature cycle (refer Equation 5.17). Once the maximum and minimum temperature ranges are evaluated, the hourly temperature is made to vary within the daily maximum/minimum range. The following analysis is made with the temperature range as indicated in Figure 7.37 below.

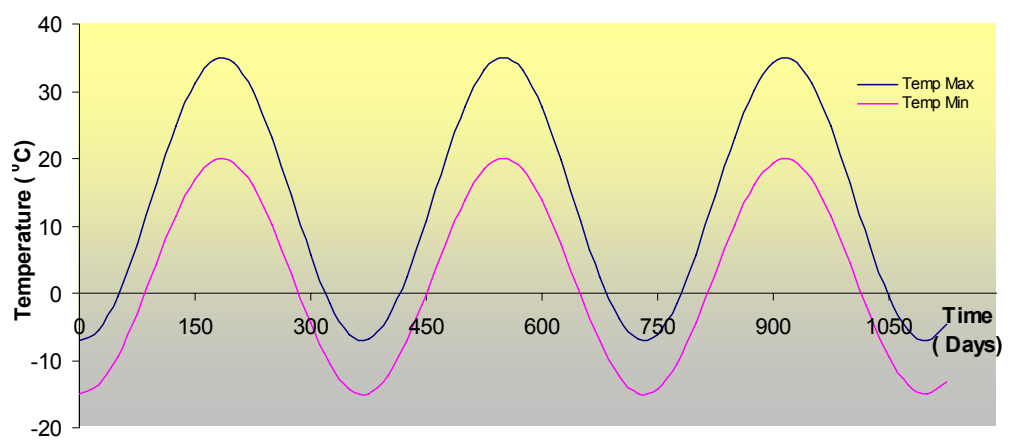

Figure 7.37 Presumed minimum and maximum annual temperature variations

As explained in subtopic 7.2.1, material input parameters are extracted from regression analysis and curve fitting approach. Typical material parameters obtained from regression analysis of data from literature (Uzarowski [2006]) are presented below. 

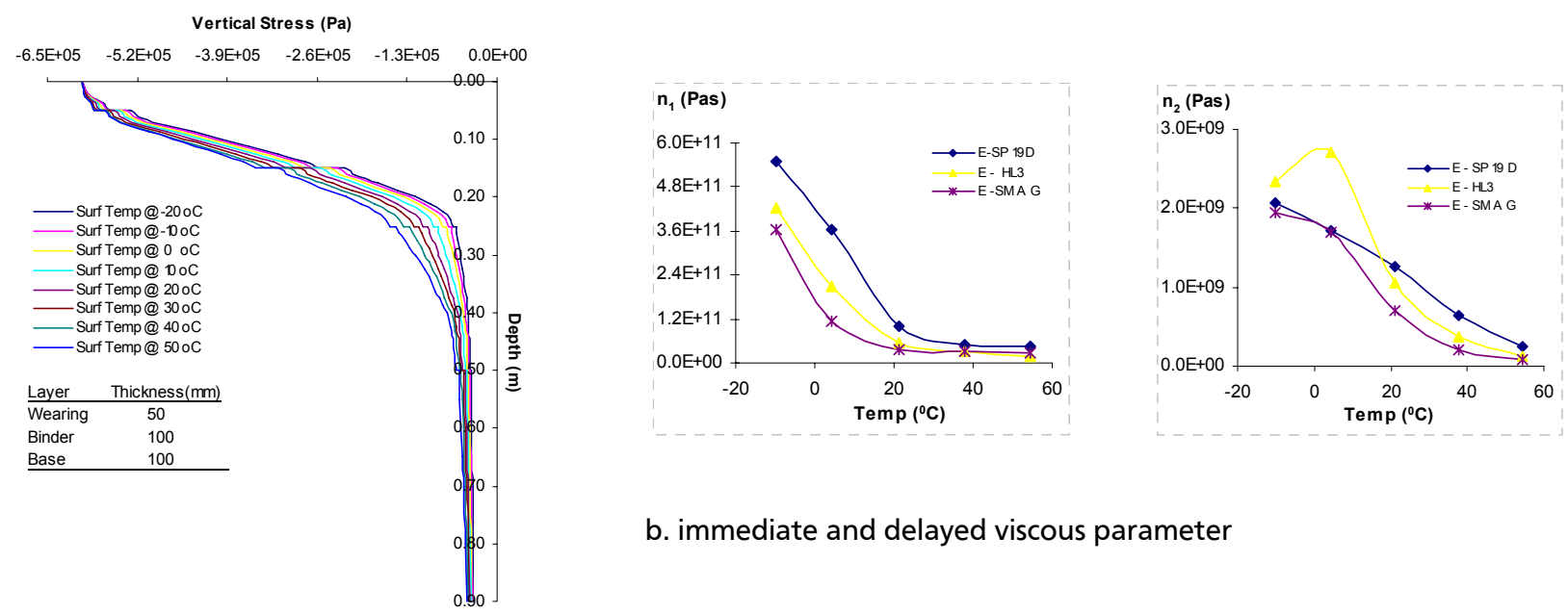

b. immediate and delayed viscous parameter

a. FE result
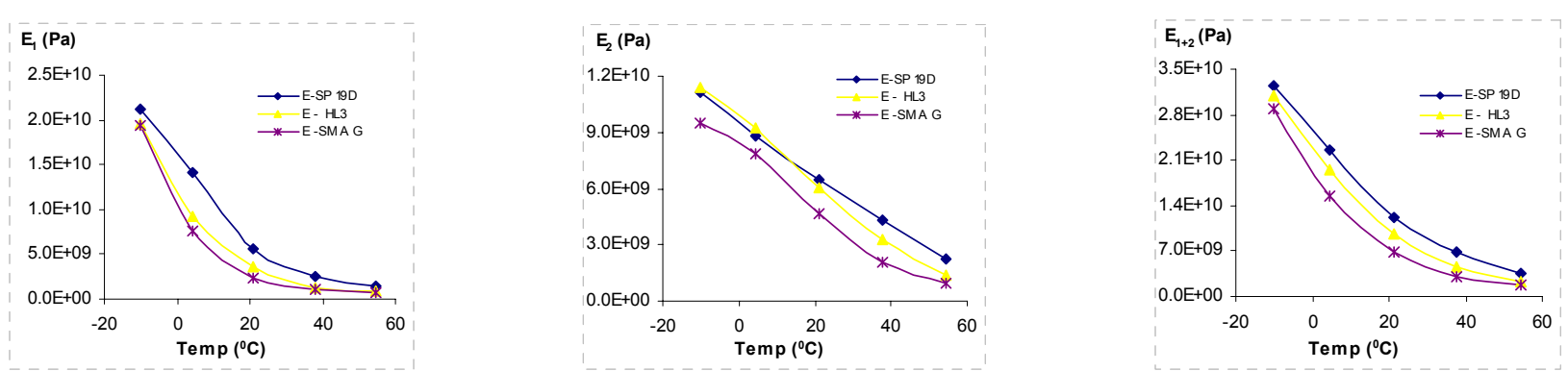

c. immediate, delayed an total elastic modulus

Figure 7.38 Material characteristics and pavement response input for model

The variations of the material parameter with respect to temperature have been expressed as indicated above. Compared to the previous result, the regression result rather show very low value with respect to the viscous parameter $\eta_{1}$ at high temperature, therefore, the deformation analysis with these parameters are made at lower traffic volume and temperature ranges. The material parameters mentioned above have been altered; particularly the immediate viscous parameter has been scaled up by five times as illustrated in Figure 7.39 below. The deformation characteristics in both material groups are given in Figure 7.40.
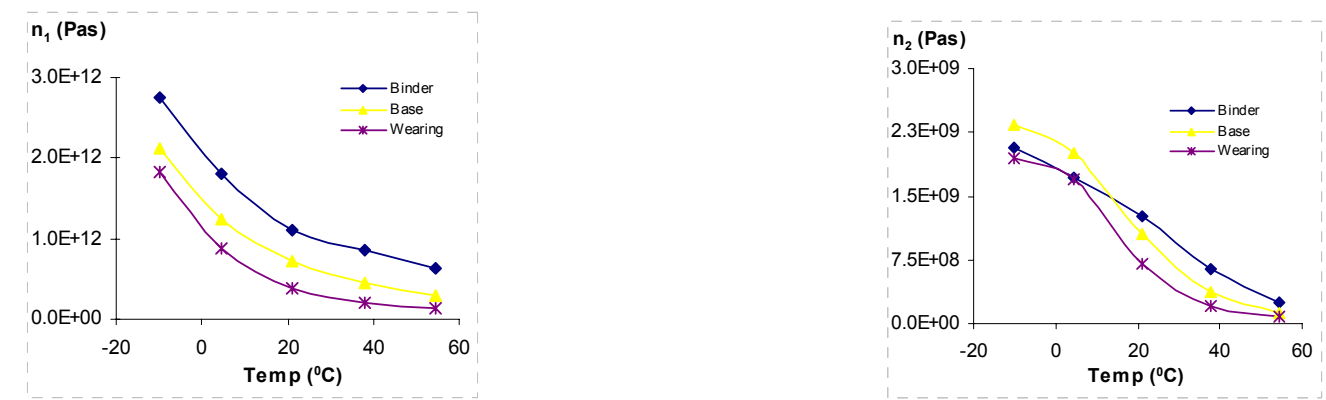

Figure 7.39 Altered viscous parameter input

At a particular time of a day, the temperature of the pavement layers is evaluated for a given annual temperature variations and hence the material property at that time and pavement responses are made known. With these material parameters the stress states are evaluated with 
ABAQUS and the mid-depth vertical stresses of the asphalt layers are applied to the burger's model to compute rutting. The time series of the load applications are determined from the traffic characteristics as explained earlier.

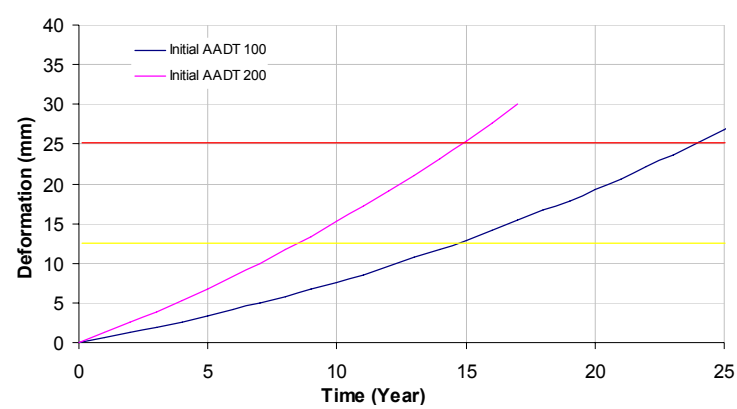

a. Deformation at without scaling up viscous parameters

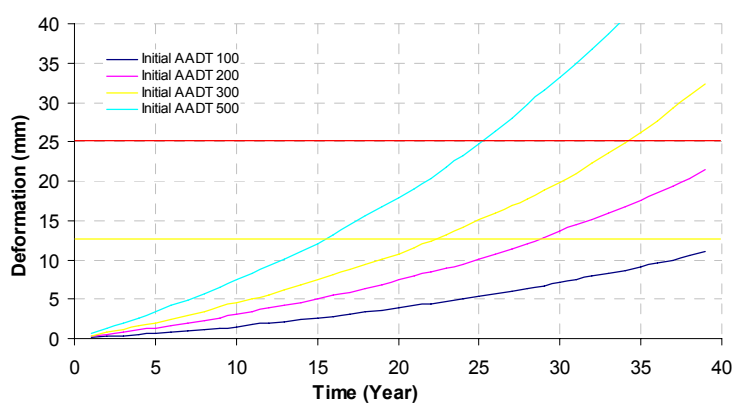

b. deformation with scaled viscous parameters

Figure 7.40 Deformation behavior at lower annual temperature and material characteristics

The deformation characteristics indicated in Figure 7.40 (a) and (b) have be computed corresponding with material parameters given in Figure 7.38 and 7.39 , respectively. The accumulation of deformation is quite significant in both cases due to lower viscous parameter. The material characteristics corresponding to Figure 7.40 (a) supports lower traffic volume for service life of less than 25 years even at low temperature range. Scaling up the viscous parameter significantly reduce the calculated deformation characteristics, let the model to accommodate more traffic volume and change corresponding service life. The model output illustrated in Figure 7.40 (b) is presumed to serve for an initial traffic volume of $300 \mathrm{cv} / \mathrm{d}$ (or equivalently 7.96 msa for about 35 years). The deformation sensitivity has been further analysed with presumed moderate temperature as indicated in Figure 7.40 (a).

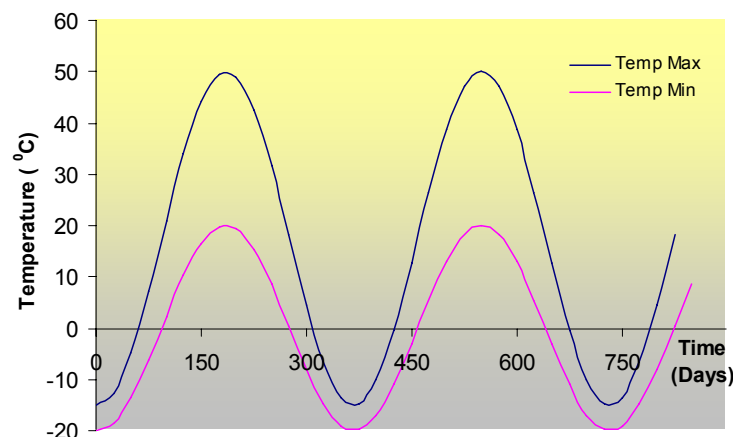

a. Presumed annual temperature variations

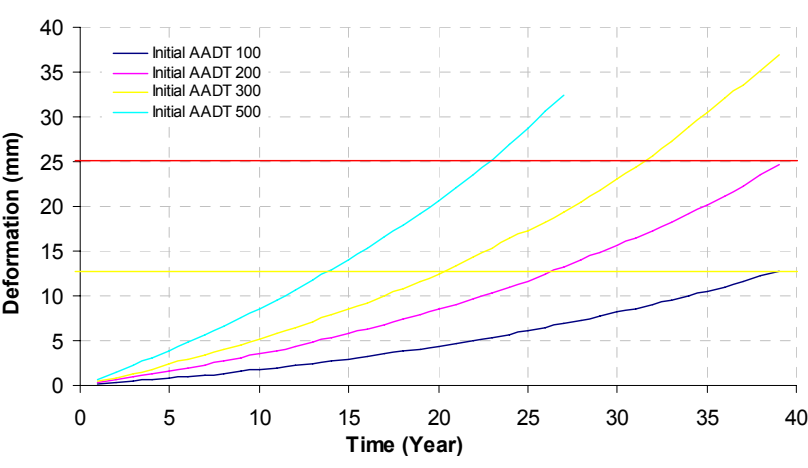

b. Deformation characteristics

Figure 7.41 Deformation behavior and with presumed temperature

Keeping the material parameter and the traffic volume the same, the deformation behaviors are relatively higher due to increase in the permanent strain at high temperature (as illustrated in Figure 7.41 (b) above). The temperature differences in both cases significantly change the service life, clearly reducing the service life with increase in temperature.

Further analyses have been made with respect to traffic volume, material parameters and temperature. The trend for deformation behavior shows that change in any of the variables greatly influences the model output. As indicated in the following figures, improving the 
immediate viscous parameter of at least 1400-2380 Gpa.s for layers exposed to high temperature would enhance the pavement sustainability for medium initial traffic streams in the range of $1000-1200 \mathrm{cv} / \mathrm{d}$ at about $4.5 \%$ growth rate or equivalently more than $27-31 \mathrm{msa}$ for service period of 35 years or more.

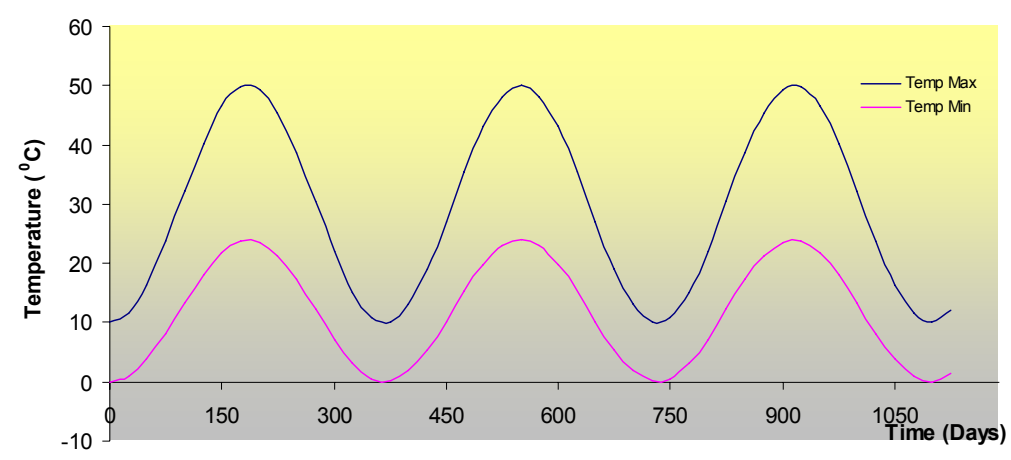

Figure 7.42 Temperature characteristics
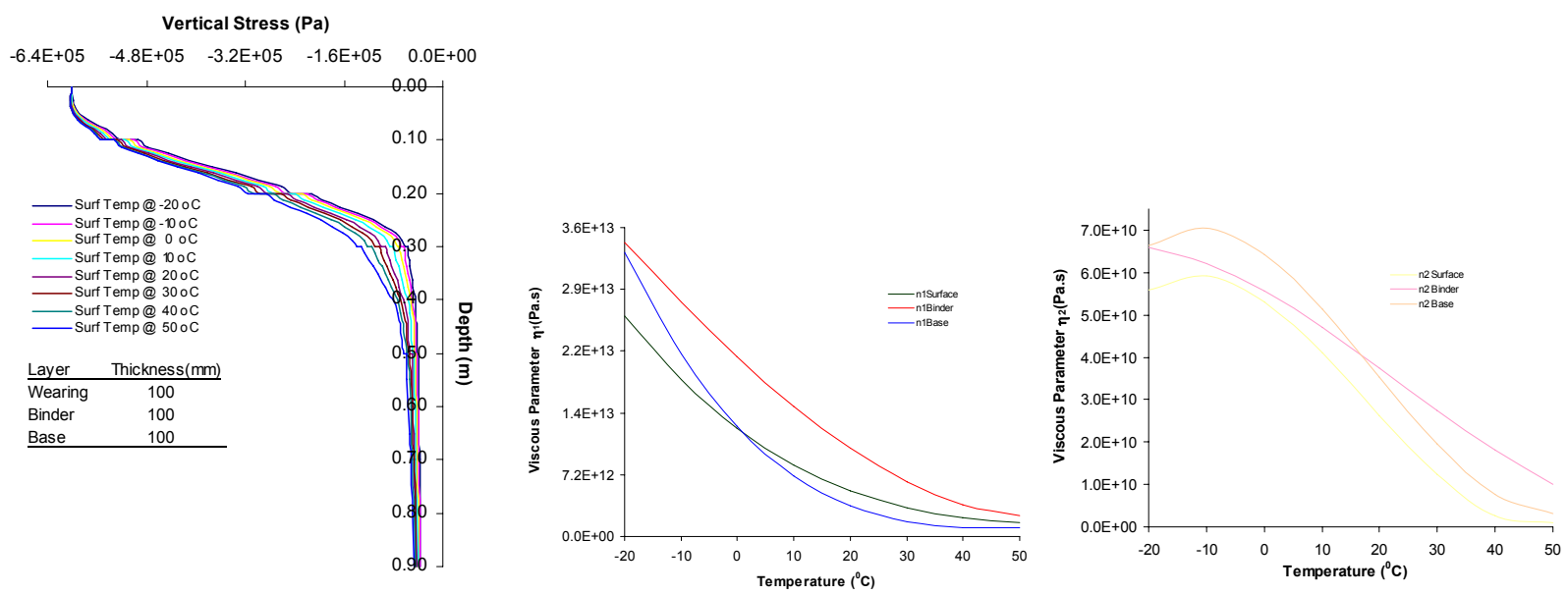

a. vertical stress

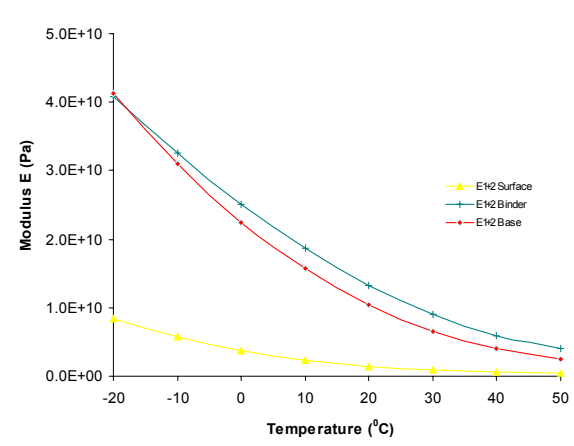

b. viscous parameters

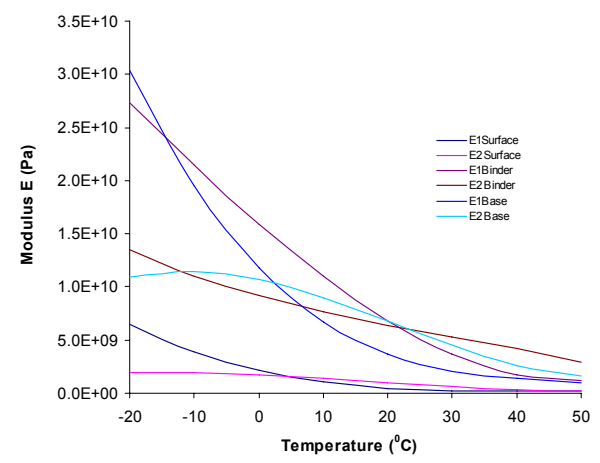

c. elastic parameters

Figure 7.43 Model input parameters 


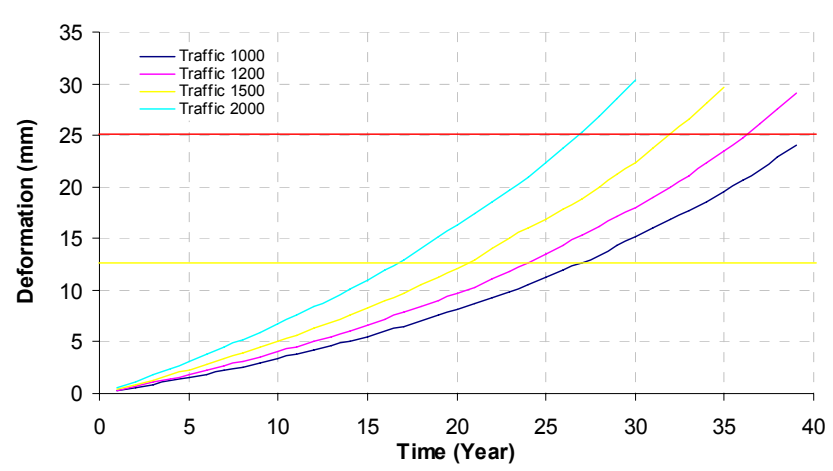

Figure 7.44 Deformation behavior with respect to traffic volume, temperature and material characteristics

It is logical that the service life of the pavement structure (with the same material and structural properties described above in Figure 7.43) drops with an increase in the ambient temperature. The following illustrates the same. When the lower temperature range raises, the deformation characteristics increases. Further analysis made for the effect of seasonal temperature variations shows the same trend.

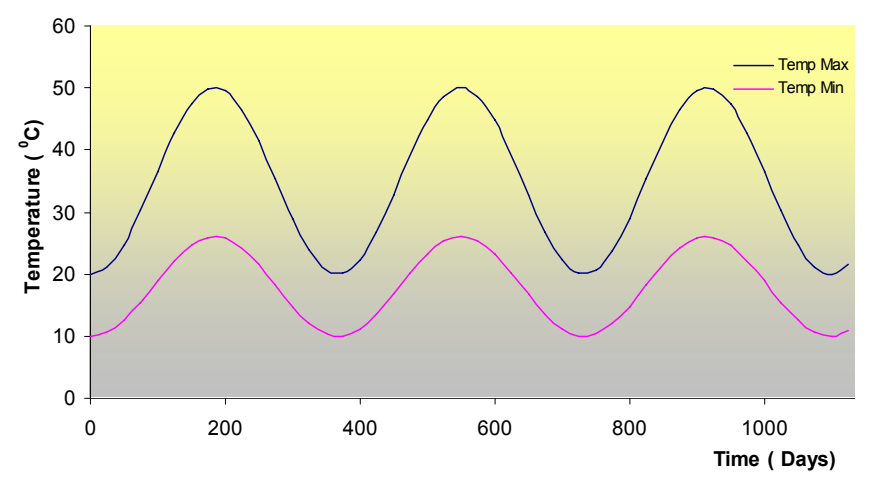

a. Temperature variations

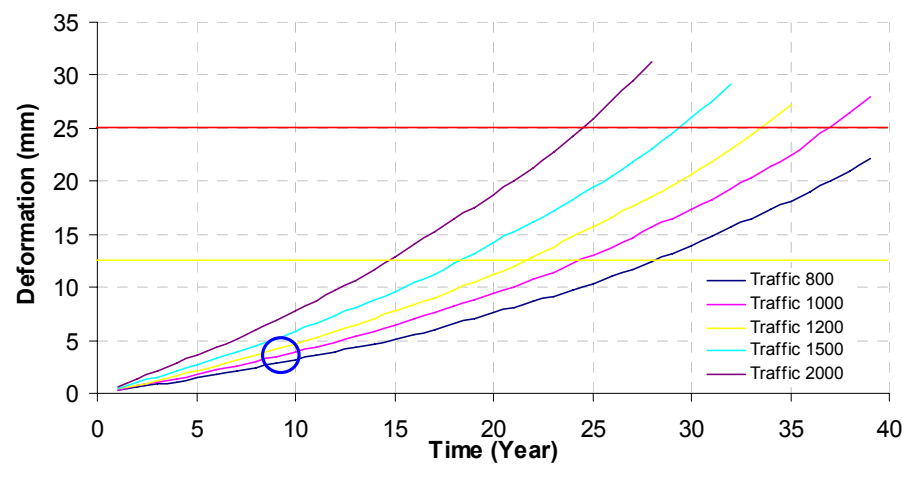

b. Deformation characteristics

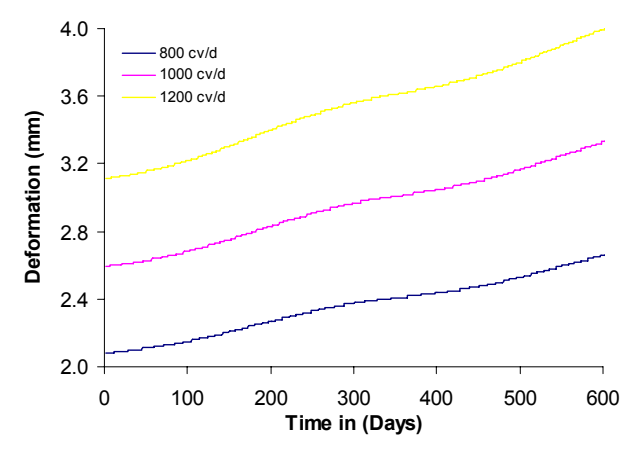

c. Seasonal deformation variations

Figure 7.45 Temperature variations and corresponding deformation behaviour

The seasonal variation of rutting is indicated in Figure 7.45 (c) (which begins at the eighth year for this case). At the beginning of the year the rate of rutting is relatively lower and the curve gradually rises, particularly the curve rises fast during summer (warmer) period (around the $200^{\text {th }}$ day) indicating the seasonal influence.

Higher traffic volume definitely requires better quality asphalt concrete layers; particularly it demands a structure of higher order magnitude of viscous parameter. Likewise on pavements 
subjected to higher ambient temperature insists to use stiffer materials with higher $\eta_{1}$ values. The combined effect of different factors in rutting, therefore, requires thorough analysis. The analysis carried out with the following reflects the effect of traffic volume and temperature and the need of provision of high quality material for asphaltic layers at higher temperature.

Additional analysis is made while keeping the elastic characteristics constant and increasing the previous viscoelastic parameters by $20 \%$. It is clearly shown that the deformation characteristics have been significantly changed. Figure 7.46 illustrates the permanent deformation with different traffic volumes. The maximum deformation of $25 \mathrm{~mm}$ is expected at the age of 33 and 38 year for initial traffic of 1500 and $2000 \mathrm{cv} / \mathrm{d}$, respectively. The pavement may serve for more than 40 years if the initial traffic is around $1000 \mathrm{cv} / \mathrm{d}$ (or equivalently for an estimated ESAL of 34.87 million at 40 years)

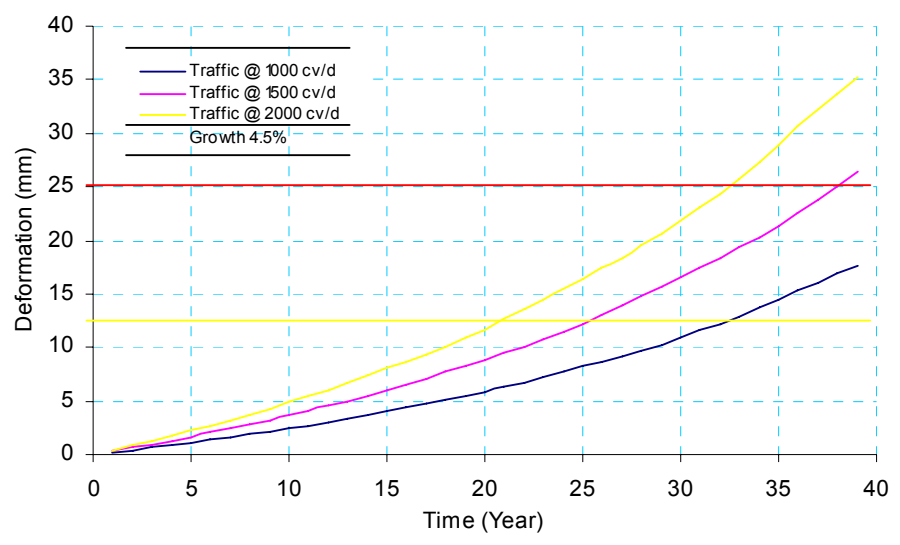

Figure 7.46 Deformation characteristics with higher $\eta_{1}$ characteristics.

The deformation is dominantly governed by the thermal sensitivity of the asphaltic layers when the pavement is exposed either to high environmental temperature, longer warmer season or when it is located in areas of high temperature zone (incase of tropical areas). In such circumstances, the viscous nature of the components of asphalt mixes has significant important. The following Figure supports the above idea. The maximum temperature has been raised to $60^{\circ} \mathrm{C}$ and corresponding material characteristics ahs been increased. The result shows that high temperature ranges requires higher magnitude of material parameters. For this typical case the lowest material parameter $\eta_{1}$ at the surface course is more than $1800 \mathrm{GPa} . \mathrm{s}$ at a maximum temperature. Such materials and corresponding thicknesses are supposed to support from 15002000 initial traffic volume for almost 40 years.

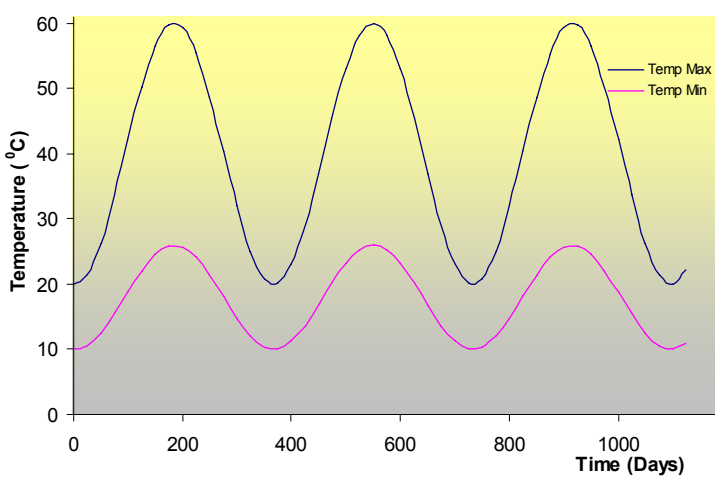

a. Annual temperature variations

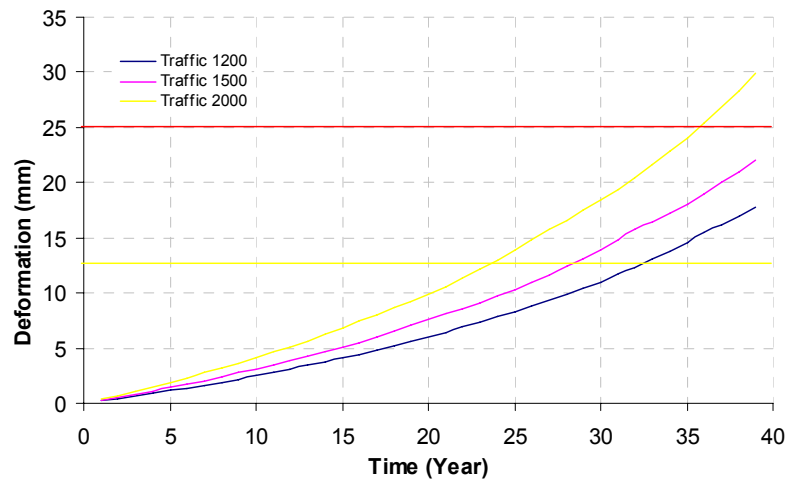

b. Rutting at higher temperatures

Figure 7.47 Temperature variations and corresponding rutting 
It has been revealed that apart from traffic characteristics, the material properties and temperature of the structural layer play a leading role in deformation characteristics. At low temperature range, the asphaltic layers are rather very stiff and their susceptibility against rutting are quite minimum. In contrast, the viscoelastic natures of the AC layers at higher temperature make them softer and more vulnerable to rapid rut formation, in such case; the thermal characteristics in AC structure have significant influence over rutting.

Apart from the applied traffic load and the environmental condition, the structural geometry and materials properties plays a leading role on the deterioration mechanism of a pavement structure. The pavement performance and its sustainability rather lie on how good the structural and materials properties are. As prediction models indicated higher viscous parameters of the asphaltic layers guarantee lower rutting rate and better performance. Results suggested that higher values of $\eta_{1}$ would reduce rutting which supports recommendations on providing a very stiff layer to reduce the effect of rutting. On the other hand thicker asphaltic layers reduce the distress developed within the substructure (Subbase and subgrade) and confine the permanent deformations within the superstructure, which allow rehabilitation to be made only on the superstructure. Therefore providing better quality of materials which addresses the problem of rutting in conjunction with optimum structural layer thicknesses would insure durable and better performance road for the community as well as for the policy maker in order to reduce potential rehabilitation and/or minimize possibility of reconstruction of the whole structure.

\subsubsection{Verifications and Uncertainties}

This work is comprised of various design and modeling elements which requires verification of their validity and applicability. Intensive laboratory investigation is required to effectively characterize the material properties at various levels of applications and different environmental circumstances. In addition, evaluation of pavement performance through field investigation is unquestionable for any validation of the office work as well as for calibration and/or analysis of discrepancies of any investigation either in the laboratory or third party technique. Accomplishing such wide range of investigations in various disciplines enhance the credibility of the research work in the damage analysis and prediction process. Further more, the research work can be redefined in such a way that the analysis and prediction process can be extended beyond the expected limit so that a reliable boundary condition can be set in order to operate and implement the result within the range of confidence.

The major variables used in this investigation include traffic characteristics, structural and material properties as well as the temperature variations. Keeping the variations and uncertainties associated with each variable in mind, an attempt is made to run the values of the elements associated to each variable within the acceptable range. Both finite element and rut prediction analyses have been based on structural geometries recommended for long life asphalt concrete pavements. In the rut prediction model, the analysis has been started with material properties obtained from literatures and upper and lower ranges of material characteristics have been set based on the predicted result corresponding to each range. Further analyses carried out within these ranges have given agreeable results.

On the other hand, it is very essential to justify predicted results with either by undergoing field investigation or compare results with recorded data. Of course the difficulties here are the availabilities of recorded data and limitations in matching with different variables. Typical example is the investigation made by Werkmeister et al. [2003] at the Dresden University on accumulation of permanent strains under long-term repeated loading conditions. The research which had taken into account the effect of temperature, moisture and stress states in the underground layers, suggested that thicker pavements (above $220 \mathrm{~mm}$ AC layers) proved to bring negligible permanent deformation in the underlined layers at higher repetitions of loading. This is taken as a partial verification of the assumptions that rut has been contributed mainly by the superstructure. 
It has also been demonstrated [73] that long life is achievable for the subsurface pavement layers and the recognition of certain surface material types that perform extremely well across a range of functional properties as well as their ability to be reliable for about 30 years of maintenancefree service life is additional justification. On the other hand, data on pavement construction, materials, and performance collected and stored in the long term pavement performance (LTPP) database are additional sources that can be utilized for verification purpose[74]. However, limiting data resources from literature have not been sufficient and flexible enough to conduct a wide range of prediction analyses and hinder further analyses beyond the expected limit. Therefore, it is valuable to carry out sound laboratory and field investigation to fill the gap and minimize the uncertainties possibly encounter during data and analysis manipulations.

A large number of structural, material, traffic characteristics as well as environmental conditions whose knowledge are required for designing structures are non-deterministic by nature and they follow a certain distribution function of probability. The use of the probability techniques to estimate the uncertainties associated to the design parameters would be beneficial to design a pavement more effectively for a particular application. Different conditions under which the measurements are made with respect to traffic characteristics, material and structural investigations, environmental conditions and other parameters involved in pavement analysis and design cause variations in the corresponding quantity and they are not absolutely determinable. Therefore an estimation of the value of the quantity would have some sort of uncertainty and there exists range of values within which the true value has to lie. In pavement damage analysis, uncertainties are inevitable from various sources and fairly defining and modelling such uncertainties are beneficiary from serviceability and performance point of view. In this report, no such analysis has been made however it is believed that the future work would be inclusive of such facts and events. 


\section{Conclusions and Recommendations}

\subsection{Conclusions}

Asphalt concrete pavement is a structure of multiple layers of different material's properties subjected to non-uniform traffic loadings and varying environmental conditions. The repetitive dynamic loads generated by heavy vehicles combined with the ever-changing temperature-field of the pavement are the primary causes of road structural damages and degradation of the performance of the pavement. Estimation and prediction of the maximum structural damages likely to happen within the pavement layer during the pavement service life is very important in order to evaluate the sustainability and durability of roads. Analytical models enable to foresee the deterioration built up taking place within the pavement structures and aid to design most economical and long lasting road.

The research work in this thesis dealt with the analysis and modeling of permanent deformation for long life asphalt concrete pavements. It has been aimed at evaluating the performance of the AC pavement due to the combined effect of expected wheel loads and temperature cycle of the pavement. There have been two major tasks carried out in this work. The primary task has been to figure out the behavior and responses of a pavement structure and associated parameters responsible for them. Modes of failures and corresponding possible causes have been reviewed. The next task has been developing and recommending a damage prediction model that can be integrated as part of a design tool for the road design industry. Among various modes of damages, rutting damage has been given priority because of the fact that some AC pavements constructed with thicker superstructure suffer due to permanent deformation while performing better against other forms of damage such as fatigue.

Analysis of permanent deformation requires evaluating the response of a pavement from different perspective. Among which; traffic loading, surrounding thermal condition as well as materials and structural characteristics play leading roles. Determining the stress-strain states in a pavement section under traffic loading is an essential primary step in damage evaluation. The estimation of traffic characteristics is a vital component of pavement life analysis and prediction as well as for assessment of pavement rutting. The effect of traffic is treated in terms of its growth rate, daily and hourly volumes, types and hourly distributions, speed as well as vehicular wheel loadings. Load spectrum concept has been followed instead of ESAL and individual wheel load has been applied while computing load associated responses. Material properties and pavement performances depend upon the rate at which they are loaded. These phenomena are evidenced by the measured material parameters as well as road sections subjected to traffic stops or congestions. Therefore the vehicle speed, where its effect is associated with loading and unloading time variables, is an important factor that greatly influences the deformation characteristics of a pavement.

The surrounding thermal environment and temperature sensitivity of the constituent materials making up the layers significantly affect the stability and long-term performance of the pavement. Prediction of the temperature profile in pavements greatly aids for assessment of pavement deflection, back-calculation of time and temperature dependent materials parameters. This requires analysis of annual and diurnal temperature cycles as well as estimation of thermal distribution in pavement layers. The property of AC changes with temperature and behaves as viscoelastic material at high temperature and elastic or stiff at low temperature. The variations in such properties are typically related to the average temperature of a layer. Therefore, the daily and annual maximum-minimum temperature variations are formulated in order to evaluate the temperature at any depth, which in turn are associated with material's properties and corresponding rutting.

The thickness of the pavement layer is the most significant part of the structural integrity of the asphalt pavement. Thickness determination of a pavement is a structural design process, 
ensuring that the thermal and traffic loads are so distributed that the stresses and strains developed at all levels of the pavement structure are within the capabilities of the materials used at those levels. The structural failure of a pavement is associated with the strength characteristics of the constituent materials and the thickness of each layer.

Pavements have to carry the heaviest anticipated heavy traffic with high axle loadings under adverse climatic conditions without excessive deformation. Long life pavements using advanced material and structural properties potentially have a great deal to offer high quality performance. The pavement layers themselves need to be strong enough to tolerate the stresses and strains to which they are exposed. Long life pavements are particularly desirable to avoid the costs of road maintenance works, including the delays they inflict on road users, particularly in congested traffic conditions.

Better understanding of material behaviors and structural responses of asphalt concrete in combination with load-stress-strain computation tools, allows for prediction the service life of a pavement as well as optimization of asphalt pavement thickness and material choice. Finite element modeling has been found very helpful in order to evaluate directly the primary pavement structural responses or beyond that without resorting to potentially costly field experiments. Further more, in order to simulate prevailing traffic and ambient temperature change simultaneously, a Burger-model-based model has been developed and programmed to predict the accumulation of rutting along the wheel path during the presumable design period of the pavement.

The responses of the pavement for various conditions have been obtained from the finite element analysis. It has been found a convenient tool for investigation of the influence of the traffic load, material properties and structural geometry on the overall responses of a structure. Different layer are created and assigned with section and material properties and the primary responses have been evaluated at presumable critical positions with varying loading characteristics, material properties and structural geometries. The finite element analysis in general has shown that higher elasticity modulus of the asphaltic layers and/or layer thickness reduces deflection as well as the stress-strain states at critical points. Critical vertical, shear and lateral stresses have been developed at the wearing, binder and base layers, respectively. It has been found that increasing the stiffness of the any layer resulted in an increase stress states of the upper layer and reduction of the same stress in lower layers.

The rutting model evaluates the accumulation of the permanent deformation for different loading spectrum and seasonal/timely variations of temperature. The vertical stress states of a pavement model evaluated by the finite element method have been employed to predict layers permanent deformations in rutting prediction model. The model was developed based on the Burgers viscoelastic principles and takes into account the change in the material properties of the structural layers due to the fluctuation of temperature and the rate of traffic loading that the pavement experiences. The program developed computes the cumulative permanent deformation through out the service life of the pavement for a given traffic and loading characteristics, temperature of the surrounding as well as structural and material properties. The number of heavy traffic loadings, operating speed characteristics and the rate of the traffic growth have significant impact on the rate of accumulation of rutting. The congestion of vehicles reduce traveling speed which incur the applied wheel load to stay over the loaded area relatively for longer period of time and also results in lower unloading time. This ultimately worsens the formation of permanent deformation. It has been found that both higher growth rate and initial traffic volume incur an increase in number of load applications and hence rapid accumulation of rutting.

Numerical investigations indicated that the viscous nature of the asphalt layer is the dominant actor to rut failure. The model output indicates that the temperature-dependent viscoelastic material parameter plays major role for the accumulation of the permanent deformation and govern the rate of deformation. The deformation behavior of the model depends on the 
magnitude of the viscous parameter of the material. How sensitive the materials properties (the viscous and elastic modulus) with respect to temperature and load matters the deformation characteristics. Especially both parameters decrease at high temperature. The higher rate of change of the viscous parameter the higher will be the rate of the deformation. For heavy duty road and in areas subjected to higher ambient temperature, it is required to select a material of highly viscous AC layers (minimum in the order of $2.0 \times 10^{3}$ GPa.s and higher) in order to achieve long life for the pavement. Therefore the quality of materials used in AC layers has to be given a thoroughly investigation and its properties have to be evaluated with respect to its viscosity as well as its sensitivity with change in temperature especially at high temperature. It has been indicated that the real problem of rutting rests on the material parameters and can be mitigated through provision of layer materials that do not yield at high temperature. Further more, mechanism of reducing such temperature sensitivity and enhancing viscosity of the material has to be sought.

AC pavement that is designed above a well defined minimum strength has a sustainable structural life provided that the distresses are kept at lower limit in such away that they don't affect structural performance of the road. Such aim can be achieved with developing the most economical combination of pavement layer thicknesses and corresponding properties that can ensure that the stresses and strains transmitted from the carriageway are reasonably below the supportive capacity limit of each layer during the entire service life of the road.

\subsection{Recommendations}

Rutting of AC is not only dependent on the compressive stress developed on the layers but also the formation of up heals on the side of the wheel track is believed to be due the shear stress developed within the layers just to the outer periphery of the projected area. It can also be seen from the finite element analysis that higher magnitude of shear stresses has been developed at the intermediate layer. The influence of the shear stress is better modeled and accommodated in rut analysis model to refine the work. In addition, calibration of the result with field result and/or previous works in this area is beneficial.

It is important to consider the effect of the stress-strain states developed within the substructure as it is distributed in a wide range of area and its influence adversely affects the subbase and subgrade layers unless these layers are strong and stable enough to mitigate the problems associated with theses states. It is well understood that moisture and underground water severely reduces the strength of these layers. Therefore it is preferable to consider such factors and evaluate the extent of the damage. It is rather advisable to model such factors for the over all performance of the pavement structure.

Asphalt concrete pavement undergoes a reduction in the stiffness as well as strength under loading and accompanied by an increase in the deterioration with time. On the other hand the aging behavior caused by the hardening of the asphalt binder under weathering, oxidation and other environmental actions displays an increase in the stiffness and strength properties with time. Even though such hardening properties seem in favor of reducing the rut formation they contribute in their own way other forms of damages. Therefore it is imperative to carefully take into account and incorporate these properties in the model.

Moisture sensitivity has been recognized as one of the major form of distress and a key element in the degradation and premature failure of asphalt concrete pavements. The loss of adhesive bond between the asphalt binder and the aggregate is due to the action of loading in the presence of moisture. Addressing distress forms associated with moisture and formulating the same would be a further step forward for analysis and modeling AC pavements.

A comprehensive model is required which include fatigue and other modes of damage including the deterioration caused by other environmental factors such as moisture and failure contribution by subgrade and subbase characteristics so that the model become fully complete 
and up to date to mitigate the complex nature of possible damage modes and associated factors. The program contains subroutines which deal about the traffic characteristics and the daily and annual fluctuation of temperature which can be integrated and implemented in fatigue and low temperature cracking model to evaluate such modes of damages during the service life of the road.

While analyzing and modeling of rutting and other forms of damages, it is very important to incorporate possible sources of uncertainties for thorough engineering judgments. Identifying, quantifying and statistically characterizing the components of AC pavements that lead to spatial variability of pavement performance can make a model more reliable and sound design tool. Therefore it is recommended to outline presumable parameters likely to influence the outcome and formulate the degree of influence of a set of parameters on the model output. 


\section{REFERENCES}

1. A Policy on Geometric Design of Highways and Streets, AASHTO, ISBN: 1-56051-156-7, Fourth Edition, Washington DC, 2001

2. ABAQUS, Abaqus Theory Manual Version 6.7, 2007

3. ABAQUS, Getting Started with ABAQUS, interactive Edition, Version 6.7, 2007

4. Abbas A. R. Simulation of the Micromechanical Behaviour of Asphalt Mixtures Using the Discrete Element Method, PhD Thesis, Washington State University, August 2004

5. Abbas A. R., A. T. Papagiannakis, and E. A. Masad, Linear and Nonlinear Viscoelastic Analysis of the Microstructure of Asphalt Concretes, Journal of Materials in Civil Engineering, ASCE, Vol. 16, No. 2, pp 133-139, April 2004

6. Ali B., M. Sadek and I. Shahrour, Finite-Element Model for Urban Pavement Rutting: Analysis of Pavement Rehabilitation Methods, Journal of Transportation Engineering, ASCE, Vol. 135, No. 4, pp 235-239, April 2009

7. Archilla A.R. and S. Madanat, Development of a Pavement Rutting Model from Experimental Data, Journal of Transportation Engineering, ASCE, Vol. 126, No. 4, pp 291299, July/August 2000

8. Archilla A.R. and S. Madanat, Estimation of Rutting Models by Combining Data from Different Sources, Journal of Transportation Engineering, ASCE, Vol. 127, No. 5, pp 379389, September/October 2001

9. Bahia H. U., D. I. Hanson, M. Zeng, H. Zhai, M. A. Khatri, R. M. Anderson, Characterization of Modified Asphalt Binders in Superpave Mix Design, NCHRP REPORT 459, TRB-National Research Council, Washington, D.C. 2001

10. Bahia H. U., Modelling of Asphalt Binder Rheology and Its Application to Modified Binders, Modelling of Asphalt Concrete, McGraw-Hill Construction, ASCE Press, pp 11-61, 2009

11. Bahuguna1 S., V. P. Panoskaltsis and K. D. Papoulia, Identification and Modeling of Permanent Deformations of Asphalt Concrete, Journal of Engineering Mechanics, ASCE, Vol. 132, No. 3, pp. 231-239, March 2006

12. Benedetto H. D., C. de La Roche, H. Baaj, A. Pronk and R. Lundström, Fatigue of Bituminous Mixtures, Materials and Structures, RILEM Technical Committees, Springer Netherlands, Vol. 37, No. 3, pp 202-216, April 2004

13. Birgisson B., A. Montepara, E. Romeo, R. Roque, R. Roncella and G. Tebaldi, Determination of Fundamental Tensile Failure Limits of Mixtures, Journal of the Association of Asphalt Paving Technologists, San Antonio, Texas, Vol. 76, pp 303 - 344, March 2007

14. Blab R. and J. T. Harvey, Modeling Measured 3D Tire Contact Stresses in a Viscoelastic FE Pavement Model, The International Journal of Geomechanics, Vol. 2, No. 3, pp 271-290, 2002

15. Borst R., Gutiérrez M. A., Wells G. N., J. J. C. Remmers and H. Askes, Cohesive-Zone Models, Higher-Order Continuum Theories and Reliability Methods for Computational Failure Analysis, International Journal for Numerical Methods in Engineering, No. 60, pp 289-315, 2004

16. Buttlar W. G., R. Roque and D. R. Hiltunen, Prediction of Thermal Cracking with TCMODEL, Modelling of Asphalt Concrete, McGraw-Hill Construction, ASCE Press, pp 405427, 2009 
17. Capros P., L. Mantzos, V. Papandreou, N. Tasios, European Energy and Transport - Trends to 2030 - Update 2007, European Commission Directorate-General for Energy and Transport, ISBN 978-92-79-07620-6, 2008

18. Cebon D., Interaction Between Heavy Vehicles and Roads, http://wwwmech.eng.cam.ac.uk/trg/publications/downloads/veh_road/veh_road11.pdf, L. Ray Buckendale Lecture, Cambridge University Engineering Department, UK, Cambridge, 1993

19. Centeno M., I. Sandoval, I. Cremades, J. Alarcón, Assessing Rutting Susceptibility of Five Different Modified Asphalts in Bituminous Mixtures Using Rheology and Wheel Tracking Test, TRB 2008 Annual Meeting CD-ROM, Washington D.C., January 2008

20. Chandra S., P. Kumar and B. A. Feyissa, Use of Marble Dust in Road Construction, International Journal of Road materials and Pavement Design, Vol. 3, No. 3, pp 317-330, 2002.

21. Chehab G. R. and Y. R. Kim, Viscoelastoplastic Continuum Damage Model Application to Thermal Cracking of Asphalt Concrete, Journal of Materials in Civil Engineering, ASCE, Vol. 17, No. 4, pp 384-392, July/August 2005

22. Chehab G. R., Characterization of Asphalt Concrete in Tension Using a ViscoElastoPlastic Model, PhD Thesis, Raleigh, North Carolina, 2002

23. Chen J. S., C. H. Lin, E. Stein and J. Hothan, Development of a Mechanistic-Empirical Model to Characterize Rutting in Flexible Pavements, Journal of Transportation Research Record, Transportation Research Record of the National Academies, ASCE, Washington D.C., Vol. 130, No 4, pp 519 - 525, July 2004.

24. Chen J. -S., M.K. Chang and K.Y. Lin, Influence of Coarse Aggregate Shape on the Strength of Asphalt Concrete Mixtures, Journal of the Eastern Asia Society for Transportation Studies, Vol. 6, pp. 1062 - 1075, 2005

25. Chen J.-S. and L.-S. Huang, Developing an Aging Model to Evaluate Engineering Properties of Asphalt Paving Binders, Materials and Structures/Mat6riaux et Constructions, Vol. 33, pp 559-565, November 2000

26. Collop A. C., A. T. Scarpas, C. Kasbergen and A. de Bondt, Development and Finite Element Implementation of a Stress Dependent Elasto-Visco-Plastic Constitutive Model with Damage for Asphalt, Paper submitted for the TRB 82nd Annual Meeting, TRB 2003 Annual Meeting CD-ROM, Washington DC, January 2003

27. Corté J. F., Development and Uses of Hard-Grade Asphalt and of High-Modulus Asphalt Mixes in France, Perpetual Bituminous Pavements, Transportation Research Circular, Washington DC, No. 503, pp 12-31, December 2001

28. Denneman E., The Application of Locally Developed Pavement Temperature Prediction Algorithms in Performance Grade ( $P g$ ) Binder Selection, Proceedings of the 26th Southern African Transport Conference (SATC 2007), ISBN N0.: 1-920-01702-X, Pretoria, South Africa, PP 257-266, July 2007

29. Deshpande V. S. and D. Cebon, Micromechanical Modeling of Steady-State Deformation in Asphalt, Journal of Materials in Civil Engineering, ASCE, Vol. 16, No. 2, pp. 100-106, April 2004

30. Dessouky S., E. Masad, D. Little and H. Zbib, Finite-Element Analysis of Hot Mix Asphalt Microstructure Using Effective Local Material Properties and Strain Gradient Elasticity, Journal of Engineering Mechanics, ASCE, Vol. 132, No. 2, pp 158-171, February 2006

31. Diefenderfer B. K., I. L. Al-Qadi and S. D. Diefenderfer, Model to Predict Pavement Temperature Profile: Development and Validation, Journal of Transportation Engineering, ASCE, Vol. 132, No. 2, pp 162-167, February 2006 
32. Drescher A., J. R. Kim and D. E. Newcomb, Permanent Deformation in Asphalt Concrete, Journal of Materials in Civil Engineering, ASCE, Vol. 5, No. 1, February 1993.

33. EAPA Heavy Duty Pavements, http://www.eapa.org/START/positionprs_publications/papers/pavements.htm

34. Elseifi M. A., I. L. Al-Qadi, and P. J. Yoo, Viscoelastic Modeling and Field Validation of Flexible Pavements, Journal of Engineering Mechanics, ASCE, Vol. 132, No. 2, pp 172-178 , February 2006

35. Epps A., Design and Analysis System for Thermal Cracking in Asphalt Concrete, Journal of Transportation Engineering, ASCE, Vol. 126, No. 4, pp 300 - 307, July/August 2000

36. ERA, Ethiopian Road Authority Manual, 2002

37. Erkens S.M.J.G., X. Liu and A. Scarpas, 3D Finite Element Model for Asphalt Concrete Response Simulation, The International Journal of Geomechanics, ASCE, Vol. 2, No. 3, pp 305-330, July 2002

38. Flexible Pavement Design Manual, Department of Transportation, State of Florida, 2002

39. Freitas E. F. D., P. Pereira, L. P. Santos and A. T. Papagiannakis. Effect of Construction Quality, Temperature, and Rutting on Initiation of Top Down Cracking, Journal of Transportation Research Record, Transportation Research Record of the National Academies, Washington D.C., Vol. 1929, pp 174 - 182, 2005.

40. Fwa T. F., S. A. Tan and L. Y. Zhu, Rutting Prediction of Asphalt Pavement Layer Using CModel, Journal of Transportation Engineering, ASCE, Vol. 130, No. 5, pp 675-683, September 2004

41. Garba R., Permanent Deformation Properties of Asphalt Concrete Mixtures, PhD Thesis, Norwegian University of Science and Technology, August, 2002

42. Ghuzlan1 K. A. and S. H. Carpenter, Traditional Fatigue Analysis of Asphalt Concrete Mixtures, Transportation Research Board, TRB 2003 Annual Meeting CD-ROM, 2003

43. Gillespie T.D., Karamihas S.M., Sayers M.W., Nasim M.A., Hansen W. and Cebon D., Effects of Heavy-Vehicle Characteristics on Pavament Response and Perfomance, NCHRP Report 353, TRB National Research Council, Washington DC, 1993

44. Harvey J. T., S. L. Weissman and C. L. Monismith, Rutting Characterization of Asphalt Concrete Using Simple Shear Tests, Modelling of Asphalt Concrete, McGraw-Hill Construction, ASCE Press, pp 269-427, 2009

45. Hassan H. F., A. Al-Nuaimi, S. Al-Oraimi, T. M.A. Jafar, Development of Asphalt Binder Performance Grades for Omani Climate, Construction and Building Materials, vol. 22, pp 1684-1690, 2008

46. Haupt P., Continuum Mechanics and Theory of Materials, ISBN 3-540-66114-x, Kassel 1999

47. http://apollo.lsc.vsc.edu/classes/met130/notes/chapter3/daily trend3.html

48. http://ec.europa.eu/transport/road/index_en.htm, 2008

49. http://training.ce.washington.edu/PGI/ Pavement Guide Interactive

50. http://whrpnews.org/06-07.htm, Wisconsin Highway Research Program - June 2007

51. http://www.flexiblepavements.org/documents/PerpPavtNewcomb 000.pdf

52. http://www.geo4va.vt.edu/A1/A1.htm Earth Temperature and Site Geology

53. http://www.gmupolicy.net/its/1-05\%20reports/VMTEstimation files/image606.jpg

54. http://www.highways.gov.uk/roads/projects/4265.aspx 
55. Hua J. and T. White, A Study of Nonlinear Tire Contact Pressure Effects on HMA Rutting, The International Journal of Geomechanics, ASCE, Vo. 2, No. 3, pp 353-376, July 2002

56. Huang X., H. Li and J. Zhang, Simulation of Rutting Behavior of Asphalt Pavement Based on Real Temperature Field, TRB 2008 Annual Meeting CD-ROM, Washington, D.C., January 2008

57. Kennett B.L.N., Introduction to Continuum Mechanics, http://samizdat.mines.edu/kennett/ Research School of Earth Sciences, Australian National University, Canberra ACT 0200, Australia

58. Khedr S. A., Deformation Mechanism in Asphaltic Concrete, The Journal of Transportation Engineering, ASCE, Vol. 112, No. 1, January 1986

59. Kim Y. R. and D. N. Little, Linear Viscoelastic Analysis of Asphalt Mastics, Journal of Materials in Civil Engineering, ASCE, Vol. 16, No. 2, pp 122 - 132, April 2004

60. Kim Y. R., D. H. Allen and D. N. Little, Damage-Induced Modeling of Asphalt Mixtures through Computational Micromechanics and Cohesive Zone Fracture, Journal of Materials in Civil Engineering, ASCE, pp 477 - 484, Sep-Oct. 2005

61. Kim Y. R., D. N. Little, P.E., and R. L. Lytton, Fatigue and Healing Characterization of Asphalt Mixtures, Journal of Materials in Civil Engineering, ASCE, Vol. 15, No. 1, pp 7583, February 2003

62. Kregting R., Cohesive Zone Models Towards a Robust Implementation of Irreversible Behaviour, www.mate.tue.nl/mate/pdfs/5169.pdf, Feb. 2005

63. Lane B., C. Raymond, T. Kazmierowski, G. Kennepohl, Comparative Long Term Performance of Canada's First SMA Freeway Project, TRB 2008 Annual Meeting CD-ROM, Transportation Research Board, Washington, D.C., January 2008

64. Lavin P. G., Asphalt Pavements, A Practical Guide to Design, Production and Maintenance for Engineers and Architects, Spon Press, Tailor \& Francis Group, published in London EC4P 4EE and New York NY 10001, ISBN 0-415-24733-0 (printed version), 2003

65. Lee H. J., J. H. Lee and H. M. Park, Performance Evaluation of High Modulus Asphalt Mixtures for Long Life Asphalt Pavements, Construction and Building Materials, Science Direct, ELSEVIER, Vol. 21, pp 1079-1087, 2007

66. Lee H. J., J. S. Daniel Y. R. Kim, Continuum Damage Mechanics-Based Fatigue Model of Asphalt Concrete, Journal of Materials in Civil Engineering, ASCE, Vol. 12, No. 2, pp 105112, May 2000

67. Lee S. J., S. N. Amirkhanian, B. J. Putman and K. W. Kim, Laboratory Study of the Effects of Compaction on the Volumetric and Rutting Properties of CRM Asphalt Mixtures, Journal of Materials in Civil Engineering, ASCE Vol. 19, No. 12, pp 1079-1089, December 2007

68. Lemaitre J. and R. Desmorat, Engineering Damage Mechanics, http://www.springerlink.com/content/q2703t2m245217g1/fulltext.pdf, ISBN 978-3-54021503-5 (Print) 978-3-540-27293-9 (Online), Springer Berlin Heidelberg, pp 1-76, 2005

69. Lermaitre J. and J.L. Chaboche, Mechanics of Solid Materials, ISBN 0-521-32853-5, Cambridge, 1990

70. Li X. and M. O. Marasteanu, Cohesive Modeling of Fracture in Asphalt Mixtures at Low Temperatures, International Journal of Fracture, Springer Netherlands, Vol. 136, No. 1-4, pp. 285-308, November 2005

71. Ling H. I. and Z. Liu, Performance of Geosynthetic-Reinforced Asphalt Pavements, Journal of Geotechnical and Geo-Environmental Engineering, ASCE, Vol. 127, No. 2, pp 177-184, February 2001 
72. Liu Y. and Z. You, Determining Burger's Model Parameters of Asphalt Materials using Creep-recovery Testing Data, Pavements and Materials 2008: Modelling, Testing, and Performance, ASCE, Minneapolis, pp 26-36, 2008

73. Long-Life Surfaces for Busy Roads, Transport Research Centre, International Transport Forum, ISBN 978-92-821-0158-2, Paris, May 2008

74. Long-Term Pavement Performance Standard Data Release 23.0, DVD Version, US Department of Transportation, Federal Highway Administration, January 2009.

75. Lundström R., U. Isacsson and J. Ekblad, Investigations of Stiffness and Fatigue Properties of Asphalt Mixtures, Journal of Materials Science, Kluwer Academic Publishers, Vol. 38, pp. 4941 - 4949, 2003

76. Masad E. and N. Somadevan, Microstructural Finite-Element Analysis of Influence of Localized Strain Distribution on Asphalt Mix Properties, Journal of Engineering Mechanics, ASCE, Vol. 128, No. 10, pp 1106-1115, October 2002

77. Matthews J. M., C. L. Monismith and J. Craus, Investigation of Laboratory Fatigue Testing Procedures for Asphalt Aggregate Mixtures, Journal of Transportation Engineering, ASCE, Vol. 119, No. 4, July/August 1993

78. McShane W. R. and R.P. Roess, Traffic Engineering, Polytechnic University, Prentice Hall Polytechnic Series in Traffic Engineering, PRENTICE HALL, Englewood Cliffs, New Jersey 07632, ISBN 0-13-926148-6, 1990

79. Mechanisches Verhalten von Tragschichten ohne Bindemittel in Bfestigungen für Verkehrsflächen, FGSV-Arbeitspaper Nr. 60, Ausgabe 2003

80. Mun S., Nonlinear Finite Element Analysis of Pavements and Its Application to Performance Evaluation, $\mathrm{PhD}$ Thesis, North Carolina State University, 2003

81. Nazarian S. and G. Alvarado, Impact of Temperature Gradient on Modulus of Asphaltic Concrete Layers, Journal of Materials in Civil Engineering, ASCE, Vol. 18, No. 4, pp 492499, August. 2006

82. Nunn M. and B. W. Ferne, Design and Assessment of Long-Life Flexible Pavements, Perpetual Bituminous Pavements, Transportation Research Circular, Washington DC, No. 503, pp 32-49, December 2001

83. O'Flaherty C.A., Highways The Location, Design, Construction and Maintenance of Road Pavements, Fourth edition, ISBN-13: 978-0-7506-5090-8, 2007

84. Park D. W., A. E. Martin and E. Masad, Effects of Non-uniform Tire Contact Stresses on Pavement Response, Journal of Transportation Engineering, ASCE, Vol. 131, No. 11, pp 873-879, November 2005

85. Park D. Y., N. Buch and K. Chatti, Effective Layer Temperature Prediction Model and Temperature Correction via Falling Weight Deflectometer Deflections, Transportation Research Record, Journal of Transportation Research Board, Transportation Research Board of the National Academies, Vol. 1764, pp. 97 - 111, 2001

86. Park H., J. Kim, Y. Kim, H. Lee, Determination of the Layer Thickness for Long-Life Asphalt Pavements, Proceedings of the Eastern Asia Society for Transportation Studies, Vol. 5, pp. $791-802,2005$

87. Park S. W. and Y. R. Kim, Fitting Prony Series Viscoelastic Models with Power-Law Presmoothing, Journal of Materials in Civil Engineering, ASCE, pp 26 - 32, April 2001

88. Pass M., Continuum Damage Mechanics with an Application to Fatigue, ISBN 90-90036881, Dutch, Eindhoven 1990 
89. Polacco G., J. Stastna, D. Biondi and L. Zanzotto, Relation Between Polymer Architecture and Nonlinear Viscoelastic Behaviour of Modified Asphalts, Current Opinion in Colloid \& Interface Science vol. 11, pp 230-245, October 2006

90. Prowell B. D., J. Zhang and E. R. Brown, Aggregate Properties and the Performance of Superpave-Designed Hot Mix Asphalt, NCHRP Report 539, TRB-National Research Council, Washington, D.C. 2005

91. Rasmussen R. O., R. L. Lytton and G. K. Chang, Method to Predict Temperature Susceptibility of an Asphalt Binder, Journal of Materials in Civil Engineering, ASCE, Vol. 14, No. 3, pp 246-252, June 2002

92. Richtlinien für die Standardisierung des Oberbaues von Verkehrsflächen, RSto 01, FGSV, Ausgabe 2001

93. Rodin G. J., Continuum Damage Mechanics and Creep Life Analysis, Journal of Applied Mechanics , ASME , Vol. 67, pp. 193-196, March 2000

94. Saad B., H. Mitri and H. Poorooshasb, Three-Dimensional Dynamic Analysis of Flexible Conventional Pavement Foundation, Journal of Transportation Engineering, ASCE, Vol. 131, No. 6, pp. 460-469, June 2005

95. Saad B., H. Mitri and H. Poorooshasb, 3D FE Analysis of Flexible Pavement with Geosynthetic Reinforcement, The Journal of Transportation Engineering, ASCE, Vol. 132, No. 5,pp 402-415, May 2006

96. Schwartz C. W. and K. E. Kaloush, Permanent Deformation Assessment for Asphalt Concrete Pavement and Mixture Design, Modelling of Asphalt Concrete, McGraw-Hill Construction, ASCE Press, pp 317-351, 2009

97. Shen S. and S. H. Carpenter, An Energy Approach for Airport Pavement Low Damage Fatigue Behavior, 2007 FAA Worldwide Airport Technology Transfer Conference, Atlantic City, New Jersey, USA, April 2007

98. Shen S. and S. H. Carpenter, Application of the dissipated Energy Concept in Fatigue Endurance Limit Testing, Journal of Transportation Research Record, Transportation Research Record of the National Academies, Washington D.C. Vol. 1929, , pp 165 - 173, 2005.

99. Shen S. and S. H. Carpenter, Development of an Asphalt Fatigue Model Based on Energy Principles, Journal of the Association of Asphalt Paving Technologists, San Antonio, Texas, Vol. 76, pp 525-574, March 2007

100. Shen W. and D. J. Kirkner, Thermal Cracking of Viscoelastic Asphalt Concrete Pavement, Journal of Engineering Mechanics, ASCE, Vol. 127, No. 7, pp.700-709, July 2001

101. Shenoy A., High temperature performance grade specification of asphalt binder from the material's volumetric-flow rate, Materials and Structures, Vol. 34, pp 629-635, December 2001

102. Siddharthan R. V., N. Krishnamenon, M. El-Mously and P. E. Sebaaly, Investigation of Tire Contact Stress Distributions on Pavement Response, Journal of Transportation Engineering, ASCE, Vol. 128, No. 2, pp 136-144, March 2002

103. Simon C., Einflüsse unterschiedlicher Bereifung der Ashsen schwerer LKW auf die Asphaltdeformation, Technische Universität München, Promotionsarbeit, ISSN 0341-5538, Heft 82, 2007

104. Soltani A., M. Solaimanian and D. Anderson, An Investigation of the Endurance Limit of Hot-Mix Asphalt Concrete Using a New Uniaxial Fatigue Test Protocol, Northeast Center of Excellence for Pavement Technology/NECEPT/, The Pennsylvania Transportation Institute, 2006 
105. Sousa J.B.,J. Craus, C. L. Monismith, Summary Report on Permanent Deformation in Asphalt Concrete, Strategic Highway Research Program, National Research Council, Washington, D.C. 1991

106. Stigh U., Continuum Damage Mechanics and the Life-Fraction Rule, Journal of Applied Mechanics, ASME, Vol 73, pp. 702-704, July 2006

107. Szydlo A. and P. Mackiewicz, Asphalt Mixes Deformation Sensitivity to Change in Rheological Parameters, Journal of Materials in Civil Engineering, ASCE, Vol. 17, No. 1, pp 1-9, January/February 2005

108. Tashman L., E. Masad, D. Little, H. Zbib, A microstructure-based viscoplastic model for asphalt concrete, International Journal of Plasticity, Vol. 21, pp 1659-1685, 2005

109. Tashman L., Microstructural Viscoplastic Continuum Model for Asphalt Concrete, PhD Thesis, Texas A\&M University, December 2003

110. Transport research, Cost 324, Long term performance of road pavements, final report of the action, European cooperation in the field of scientific and technical research, Belgium, 1997

111. Transport Research, Cost 333, Development of New Bituminous Pavement Design Method, Final Report of the Action, European Cooperation in the Field of Scientific and Technical Research, Belgium, 1999.

112. Uzan J. Permanent Deformation in Flexible Pavements, Journal of Transportation Engineering, ASCE, Vol. 130, No. 1, pp 6-13, January 2004

113. Uzan J., Viscoelastic-Viscoplastic Model with Damage for Asphalt Concrete, Journal of Materials in Civil Engineering, ASCE, Vol. 17, No. 5, pp 528-534, October 2005

114. Uzarowski L., The Development of Asphalt Mix Creep Parameters and Finite Element Modeling of Asphalt Rutting, PhD Thesis, University of Waterloo, Canada, Waterloo 2006

115. Valoroso N., Theory and implementation of plasticity and viscoplasticity, Class Notes, Istituto ITC - CNR,Roma, Italy, March, 2002

116. Von Quintus H. L., Hot-Mix Asphalt Layer Thickness Design for Longer-Life Bituminous Pavements, Perpetual Bituminous Pavements, Transportation Research Circular, Washington DC, No. 503, pp 66-78, December 2001

117. Vormwald M., Materialmodellierung, Vorlesungsmitschrift, TU-Darmstadt, Fachgebiet Werkstoffmechanik, Darmstadt, 2006

118. Wagoner M.P., W.G. Buttlar and G.H. Paulino, Disk-shaped Compact Tension Test for Asphalt Concrete Fracture, Society for Experimental Mechanics, Vol. 45, No. 3, pp 270-277, June 2005

119. Walubita L. F., T. Scullion, J. Leidy, Texas Perpetual Pavements: Modulus Characterization of the Rut-Resistant HMA Mixes, TRB 2008 Annual Meeting CD-ROM, Washington, D.C., January 2008

120. Wei H., Q. Zhendong, C. Gang and Y. Jun, Epoxy asphalt concrete paving, on the deck of long-span steel bridges, Chinese Science Bulletin Vol. 48 No. 21, pp 2391 - 2394, November 2003

121. Wellner H.F. and S. Kayser, Grundlagen zur Erfassung der Temperaturbedingungen fuer eine analytische Bemessung von Asphaltbefestigungen, Forschung Strassenbau und Strassenverkehrs-technik, Heft 996, Bonn, Mai 2008

122. Werkmeister S., R. Numrich, A. R. Dawson and F. Wellner, Design of Granular Pavement Layers Considering Climatic Conditions. In Transportation Research Record: Journal of the 
Transportation Research Record, No. 1837, Transportation Research Board of the National Academies, Washington D.C., pp. 61-70, 2003

123. White T. D., J. E. Haddock, A. J. T. Hand and H. Fang, Contributions of pavement structural layers to rutting of hot mix asphalt pavements, Transportation Research Board, National Research Council, National Academy Press, NCHRP Report 468, ISBN 0309/06721-9, Washington D.C. 2002

124. Widyatmoko I., C. Ellis and J. M. Read, Energy Dissipation and the Deformation Resistance of Bituminous Mixtures, Materials and Structures, Vol. 32, pp 218-223, April 1999

125. Wong W. G. and Y. Zhong, Flexible Pavement Thermal Stresses with Variable Temperature, Journal of Transportation Engineering, Vol. 126, No. 1, pp 46-49, January/February 2000

126. Xiao Y. C., S. Li and Z. Gao, A continuum damage mechanics model for high cycle fatigue, International Journal of Fatigue, Elsevier Science Ltd., Vol. 20, No. 7, pp. 503-508, 1998

127. Yang S., C. Lu, F. Zhao, R. Reel and J. D. O'Hara, Alternative Traffic Seasonal Factor Estimation for Urban Roads in Florida, TRB 2009 Annual Meeting CD-ROM, Washington DC, January 2009

128. Yavuzturk C., K. Ksaibati and A. D. Chiasson, Assessment of Temperature Fluctuations in Asphalt Pavements Due to Thermal Environmental Conditions Using a Two-Dimensional, Transient Finite-Difference Approach, Journal of Materials in Civil Engineering, ASCE, Vol. 17, No. 4, pp 465-475, August 2005

129. Yin H., Solaimanian M., T. Kumar and Stoffels S., The Effect of Loading Time on Flexible Pavement Dynamic Response: A Finite Element Analysis, Mechanics of Time-Dependent Materials, Springer Netherlands, Volume 11, Numbers 3-4, pp 265-288, January 2008

130. Yoon C. and Allen D. H. Damage Dependent Constitutive Behavior and Energy Release Rate for a Cohesive Zone in a Thermo Viscoelastic Solid, International Journal of Fracture, Kluwer Academic Publishers, Printed in the Netherlands , pp. 55-74, 1999

131. Zhang Z. and I. Damnjanović, Applying Method of Moments to Model Reliability of Pavements Infrastructure, Journal of Transportation Engineering, ASCE, Vol. 132, No. 5, pp 416-424, May 2006 


\section{Lists of Tables}

Table 2.1 Speed limit in some European countries [Capros et al. 2008] .................................. 16

Table 2.2 Annual growth rate in EU-27 [Capros et al. 2008] .................................................. 21

Table 3.1 Results of wheel tacking test on hard grade asphalt concrete mix [Lee et al. 2007].. 35

Table 4.1 Fracture mechanics versus continuum damage mechanics [Lee et al. 2000] .............58

Table 4.2 Comparison of asphalt binder properties [Lee et al. 2007] ....................................67

Table 5.1 Values of A and B for different surface temperature [Wellner and Kayser 2008] ....... 76

Table 6.1 Rheological parameters for pavement courses (roadway surface temp. $45^{\circ} \mathrm{C}$ ) [Szydlo

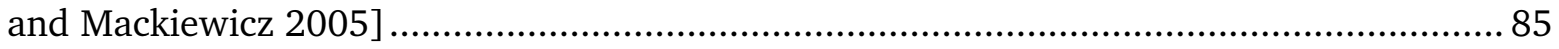

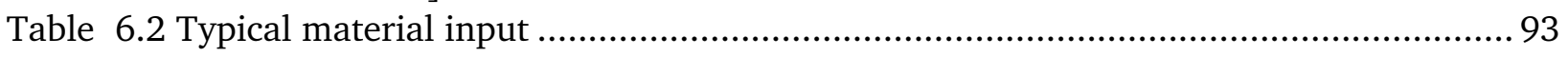

Table 7.1 Summary of dynamic modulus investigated by Uzarowski [Uzarowski 2006] ..........113

Table 7.2 Summary of regression results for SMA G mix ..................................................... 114

Table 7.3 Traffic characteristics and forecast at different period time.................................... 119 


\section{Lists of Figures}

Figure 2.1 Simplified AC pavement analysis and design strategy [modified, Ref. 38] .............. 10

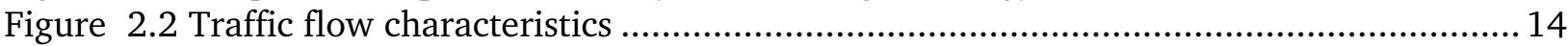

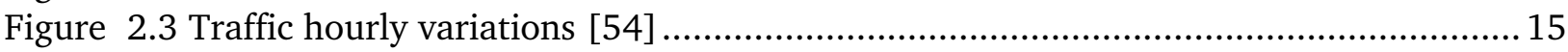

Figure 2.4 Dependency of the duration of moving load on vehicle speed and depth [Yin et al. 2008]

Figure 2.5 Variation of shear strain and shear stress with vehicle speed for dual and wide-base tires on thick pavement [Siddharthan et al. 2002] ............................................. 17

Figure 2.6 Vertical stress distribution with different magnitudes of contact pressure and wheel load

Figure 2.7 Relationship between tire and contact pressure ..............................................20

Figure 2.8 Traffic growth in EU27 [Capros et al. 2008] ........................................................ 21

Figure 2.9 Typical heavy vehicle type and axle configuration [53] ....................................... 22

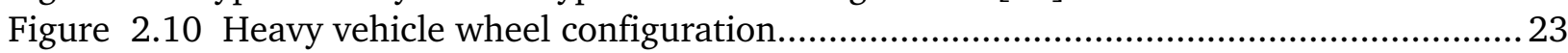

Figure 2.11 Normally distributed lateral wander of traffic wheel.........................................24

Figure 2.12 Influence of lane width on wheel path distribution [White et al. 2002] ................24

Figure 2.13 Influence of number of lanes on wheel path distribution [White et al. 2002] ....... 25

Figure 2.14 Plot and least-squares fit for example binder [Rasmussen et al. 2002] ..................26

Figure 2.15 Effect of filler on compressive strength of asphalt concrete mixtures [Chandra et al. 2002]

Figure 2.16 APA test results for four mixtures with $4 \pm 0.5 \%$ air void contents [Lee et al. 2007] 30

Figure 2.17 Stress strain curves for epoxy asphalt and hard bitumen at different temperatures [73].

Figure 3.1 Mechanism of pavement structural failures.................................................... 31

Figure 3.2 Effect of temperature on shear parameters [Bahia 2009]........................................ 32

Figure 3.3 Permanent deformation at bus stop (TU-Lichtwiese, Feb. 2009) .............................. 33

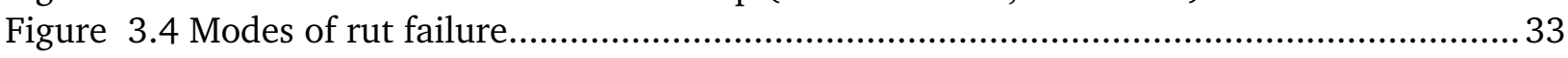

Figure 3.5 Rutting behavior with time …............................................................................. 34

Figure 3.6 Cumulative rut depths with number of load applications for conventional mix and HMAM [Lee et al. 2007]

Figure 3.8 Results of fatigue test obtained from the full-scale performance test [Lee et al. 2007]

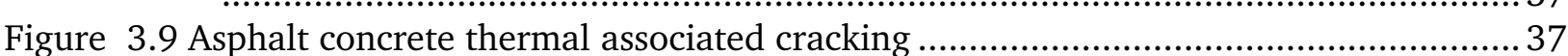

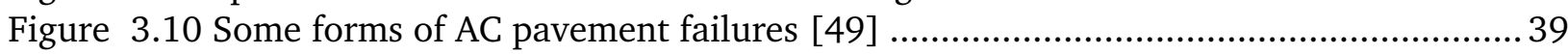

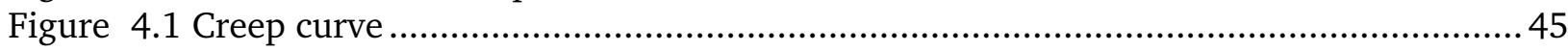

Figure 4.2 Simplified material models ................................................................... 46

Figure 4.3 Single-step creep test: (a) Loading/unloading history; (b) Strain-time response

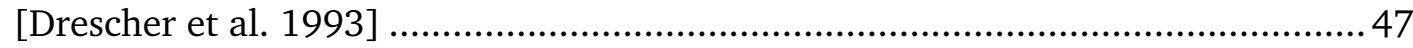

Figure 4.4 Effect temperature on viscoelastic response of a materials ................................... 48

Figure 4.5 Glass transition with respect to temperature .................................................... 48

Figure 4.6 Storage modulus as a function of reduced frequency [Chehab 2002] ..................... 49

Figure 4.7 Log shift factor as a function of temperature obtained by constructing the storage modulus master curve at $25^{\circ} \mathrm{C} \quad\left(-10,5,15,25,35^{\circ} \mathrm{C}\right)$ [Chehab 2002].................. 50

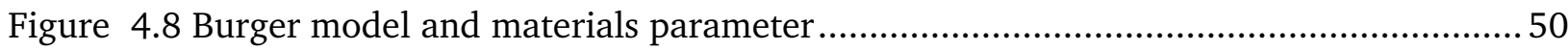

Figure 4.9 Elastic-plastic Model [Vormvald 2006] …......................................................... 51

Figure 4.10 The von Mises yield function in the deviatoric space ........................................ 52

Figure 4.11 Accumulated plastic strain on pavement surface under continuous traffic loading [Chen et al. 2004].

Figure 4.12 Schematic representation of strain components in an elasto-visco-plastic material

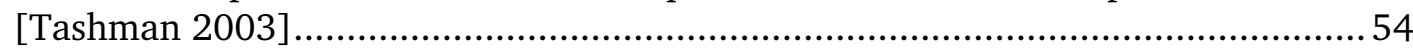

Figure 4.13 Material viscoelastoplastic model................................................................. 54

Figure 4.14 Measurement of damage by means of elasticity change [Lemaitre and Desmorat, 2005] 
Figure 4.15 Schematic representation damage [Lemaitre and Desmorat, 2005]

Figure 4.16 Representation of crack initiation and propagation ............................................5 57

Figure 4.17 Fracture modelling and testing of asphalt concrete specimen [Wagoner et al. 2005]

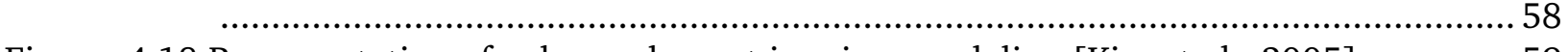

Figure 4.18 Representation of volume element in micro modeling [Kim et al. 2005] ..............59

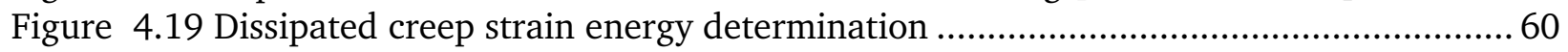

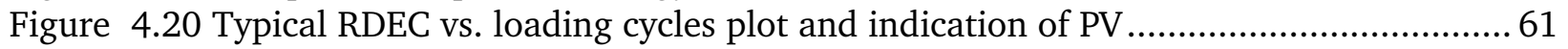

Figure 4.21 Stiffness vs. loading cycles curve [Shen and Carpenter 2007] ............................... 62

Figure 4.22 Rut depth at surface of the UGL as function of N (11.5 t-axle load); asphalt

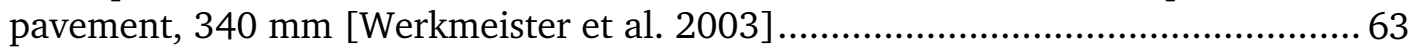

Figure 4.23 Normal fatigue testing results versus endurance limit testing [51] .......................64

Figure 4.24 Schematic of loading in Stages I, II, and III [Soltani et al. 2006] ..........................66

Figure 4.25 Comparison of rut depths for conventional mixtures and HMAM pavement [Lee et

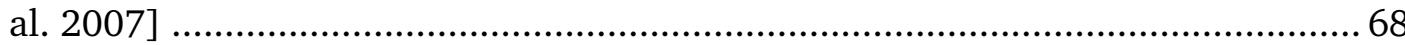

Figure 5.1 Energy balance on asphalt concrete pavement surface..................................... 72

Figure 5.2 Time lag between the maximum temperature of pavement layer and air [47] ........ 73

Figure 5.3 Annual temperature fluctuations in asphalt pavements [52] ............................... 73

Figure 5.4 Temperature history at a given depths and amplitude [Wellner and Kayser 2008] . 77

Figure 5.5 Temperature gradient for defined surface temperatures [Wellner and Kayser 2008]

Figure 5.6 Vertical temperature gradient for various days and times for different temperature ranges (station P865-Inntal Dreieck) [Wellner and Kayser 2008] ........................ 77

Figure 5.7 Variation in modulus and impact of layer thickness during heating and cooling cycles

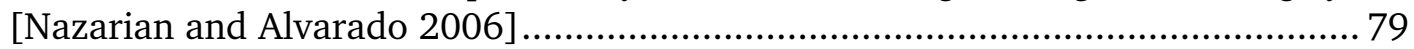

Figure 5.8 Impact of temperature gradient on modulus [Nazarian and Alvarado 2006]............ 79

Figure 5.9 Measured temperatures at surface and mid-depth versus AC modulus [Park et al.

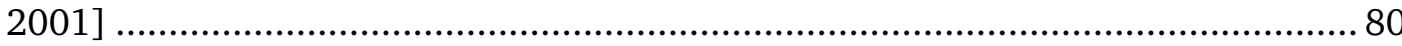

Figure 5.10 Rut depth variations with change in temperature and rate of loading [Fwa et al.

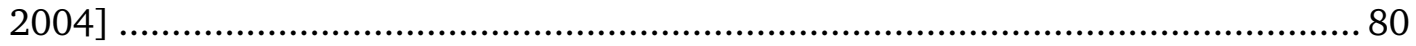

Figure 6.1 Orientation of principal stresses with respect to position of moving load ................ 81

Figure 6.2 Rut accumulation at different with wander differences [Hua and White 2002] ....... 83

Figure 6.3 Permanent strain and strain rate versus the number of loading cycles..................... 83

Figure 6.4 Plot of permanent strain curve as produced by the dynamic creep test [Widyatmoko et al. 1999] …................................................................................................ 84

Figure 6.5 Plot of dissipated energy per cycle $\left[\mathrm{J} / \mathrm{m}^{3}\right]$ against the number of load repetitions

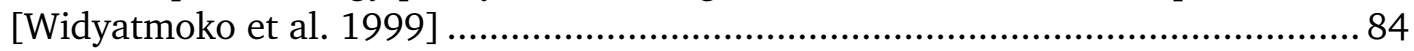

Figure 6.6 Creep curve approximation by burger model [Szydlo and Mackiewicz 2005].......... 85

Figure 6.7 Relationships between wearing course rheological parameters and calculated rut depth [Szydlo and Mackiewicz 2005] ............................................................... 85

Figure 6.8 Relationships between binder course rheological parameters and calculated rut depth [Szydlo and Mackiewicz 2005] ....................................................................... 86

Figure 6.9 Finite element modeling of asphalt concrete pavement ..................................... 88

Figure 6.10 Critical stress states within the pavement structure with FE analysis ..................... 88

Figure 6.11 Comparison between measured and calculated (a) Longitudinal strain at the bottom of base course (viscoelastic); and (b) Vertical stress at the bottom of the wearing surface [Elseifi et al. 2006]

Figure 6.12 FE 2D model with single and multiple load cases ........................................ 91

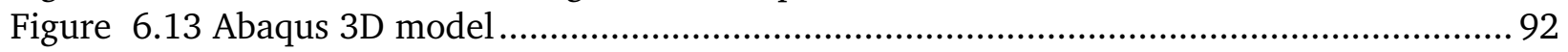

Figure 6.14 Abaqus 3D model with multiple load case ........................................................ 92

Figure 6.15 Material parameters for viscoelastic analysis ...................................................... 93

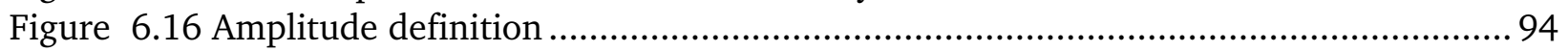

Figure 6.17 FE field output in the visualization module......................................................... 94

Figure 6.18 Result analysis with reference to selected path ............................................ 95

Figure 6.19 Output analysis with respect to applied pressure at selected depths ...................... 96 
Figure 7.1 Instantaneous deflection behavior with change in AC thicknesses ......................... 98

Figure $7.2 \mathrm{FE}$ vertical stress out put in AC layer................................................................. 99

Figure 7.3 Vertical stress distribution on selected vertical path from centre of load application 99

Figure 7.4 Vertical stress variations w.r.t. different binder and base E-modulus ..................... 100

Figure 7.5 3D lateral stress-strain state due multiple wheel loads...................................... 101

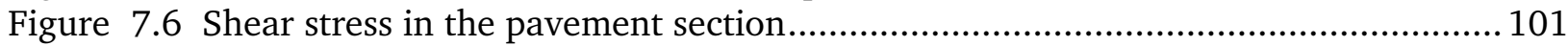

Figure 7.7 Shear stresses variations in selected vertical path ........................................... 102

Figure 7.8 Vertical stress variations w.r.t. different binder and base E-modulus ..................... 102

Figure 7.9 Stress state under dual wheel load application .............................................. 103

Figure 7.10 Influence of the tire configuration on the distribution of vertical stresses .......... 103

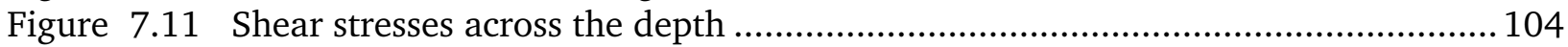

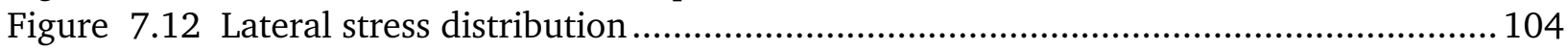

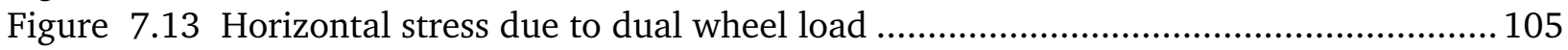

Figure 7.14 Vertical stresses and strains for different asphalt layer thickness ...................... 105

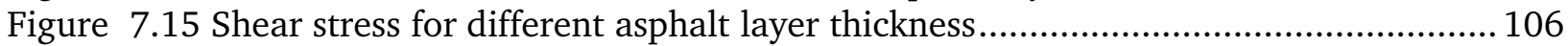

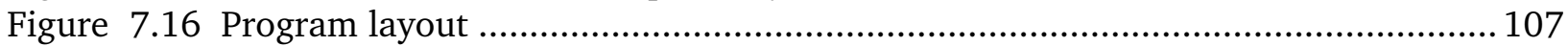

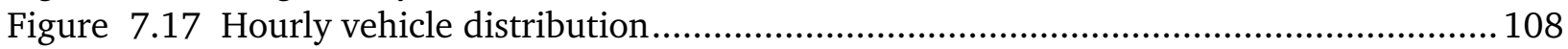

Figure 7.18 Representation of vehicular characteristics in the program............................. 108

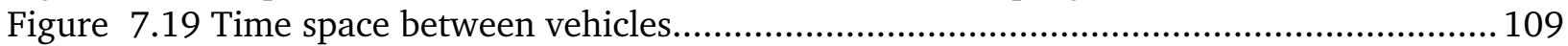

Figure 7.20 Systematic representation of wheel load application with time ......................... 109

Figure 7.21 Normal stress vs. load duration due to speed variation ................................... 110

Figure 7.22 Time space variations with respect to traffic speed ......................................... 110

Figure 7.23 Annual temperature fluctuation pattern.................................................... 111

Figure 7.24 Daily temperature variations at selected date .............................................. 111

Figure 7.25 Schematic representation of temperature gradient across the depth of a pavement

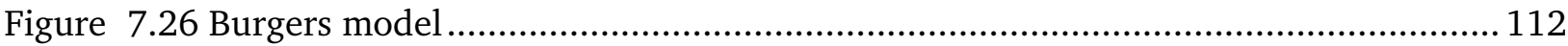

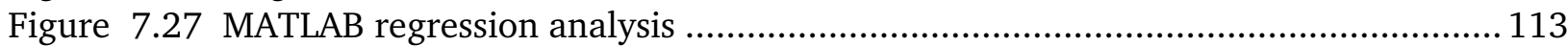

Figure 7.28 Burgers parameters variations with respect to temperature for SMA G mix ......... 114

Figure 7.29 Elastic modulus change with respect to temperature ...................................... 115

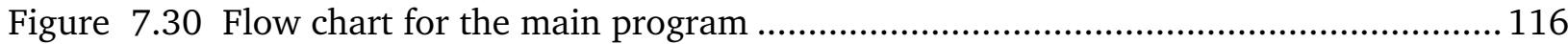

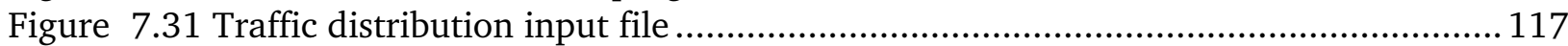

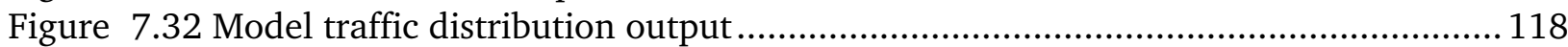

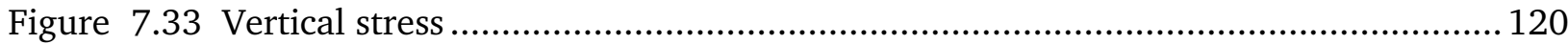

Figure 7.34 Deformation characteristics with respect to traffic volume and materials

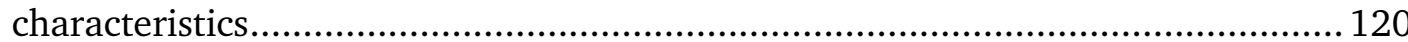

Figure 7.35 Deformation characteristics with respect to traffic volume and materials

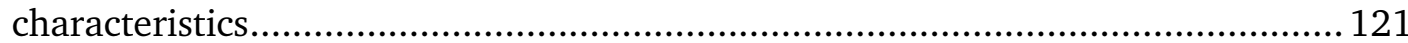

Figure 7.36 Trend of AADT with rates of growth and corresponding permanent deformation. 121

Figure 7.37 Presumed minimum and maximum annual temperature variations..................... 122

Figure 7.38 Material characteristics and pavement response input for model........................ 123

Figure 7.39 Altered viscous parameter input............................................................... 123

Figure 7.40 Deformation behavior at lower annual temperature and material characteristics 124

Figure 7.41 Deformation behavior and with presumed temperature.................................. 124

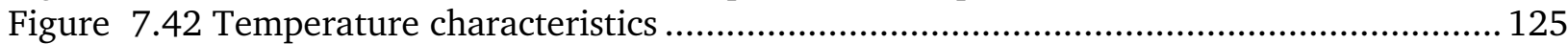

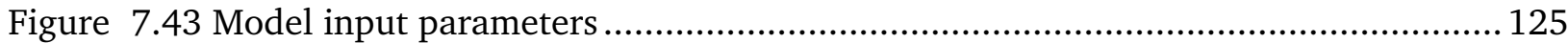

Figure 7.44 Deformation behavior with respect to traffic volume, temperature and material

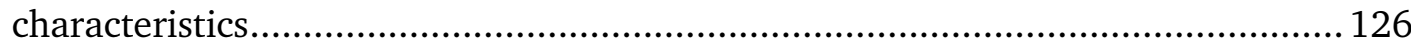

Figure 7.45 Temperature variations and corresponding deformation behaviour..................... 126

Figure 7.46 Deformation characteristics with higher $\eta_{1}$ characteristics................................ 127

Figure 7.47 Temperature variations and corresponding rutting ..................................... 127 


\begin{tabular}{ll}
\hline Notations & \\
\hline 3PB & Three Point Bending Beam Test \\
AADT & Annual Average Daily Traffic \\
AASHTO & American Association of State Highway and Transportation Officials \\
AC & Asphalt concrete \\
ADT & Average Daily Traffic \\
APT & Accelerated Pavement testing \\
AVI & Automated Vehicle Identification \\
CBR & California Bearing Ratio \\
CZM & Cohesive Zone Model \\
ERA & The Ethiopian Road Authority \\
ESAL & Equivalent single axle loads \\
EU & European Union \\
FE & Finite element \\
FEA & Finite Element Analysis \\
FEM & Finite Element Method/Model \\
FEP & Finite element program \\
GDP & Gross Domestic Product \\
HAMB & High Modulus Asphalt Binder \\
HMA & Hot Mix Asphalt \\
HMAC & High Modulus Asphalt Concrete \\
HMAM & High Modulus Asphalt Mix \\
HV & Heavy vehicle \\
IRC & The Indian Road Congress \\
IT & Indirect tensile \\
LTPP & Long Term Perpetual Pavement \\
msa & million single axle \\
NLCD & nonlinear continuous damage \\
OGFC & Open Graded Friction Course \\
PCC & Portland Cement Concrete \\
PG & Penetration Grade \\
PMA & Polymer modified asphalt \\
RVE & representative volume element \\
SBS & styrene-butadiene-styrene polymer \\
SCB & semi circular bend \\
SMA & Stone mastic asphalt \\
SN & Structural Number \\
TRB & Transport Research Board \\
VECD & viscoelastic continuum damage model \\
VMA & Voids in the Mineral Aggregate \\
WIM & Weigh-in-motion \\
WLF & William, Landal and Ferry \\
&
\end{tabular}




\begin{tabular}{|c|c|}
\hline $\overrightarrow{\mathrm{n}}$ & vector normal to a plane with surface density of micro defect \\
\hline$\widetilde{\overrightarrow{\vec{n}}}$ & vector normal to a transformed plane of micro defect \\
\hline$\varepsilon_{\mathrm{i}}^{\mathrm{p}}$ & average permanent (plastic) strain in the $\mathrm{i}^{\text {th }}$ sublayer, \\
\hline$\tilde{\sigma}$ & effective stress \\
\hline$\widetilde{\mathrm{E}}_{\mathrm{ijkl}}$ & elasticity tensor softened by damage \\
\hline$\dot{\varepsilon}^{\mathrm{p}}$ & irreversible plastic strain rate vector \\
\hline$\dot{\phi}$ & rate of volumetric strains \\
\hline$\dot{\varepsilon}^{\mathrm{e}}$ & reversible elastic strain rate vector \\
\hline$\theta$ & temperature coefficient \\
\hline$\phi$ & mechanical volumetric strains \\
\hline$\tau$ & reduced time \\
\hline$\tau$ & relaxation time \\
\hline$\xi$ & reduced time at reference temperature \\
\hline$\varepsilon$ & strain \\
\hline$\sigma$ & stress \\
\hline$\phi$ & yield Function \\
\hline$\varepsilon_{0}$ & initial strain \\
\hline$\varepsilon_{\mathrm{D}}$ & viscous strain component \\
\hline$\sigma_{\mathrm{D}}$ & viscous stress component \\
\hline$\varepsilon^{\mathrm{e}}$ & elastic strain \\
\hline$\varepsilon_{\mathrm{E}}$ & elastic strain component \\
\hline$\sigma_{\mathrm{E}}$ & elastic stress component \\
\hline$\varepsilon^{\mathrm{el}}$ & elastic strain \\
\hline$\varepsilon f$ & failure strain \\
\hline$\sigma_{\mathrm{ij}}$ & stress \\
\hline$\varepsilon_{\mathrm{p}}$ & permanent strain \\
\hline$\varepsilon^{\mathrm{pl}}$ & plastic strain \\
\hline$\varepsilon_{\mathrm{t}}$ & tensile strain in asphalt concrete \\
\hline$\varepsilon_{\mathrm{V}, \mathrm{AC}}$ & vertical strain in asphalt concrete \\
\hline$\varepsilon_{\mathrm{V}, \text { subg }}$ & vertical strain in subgrade \\
\hline$\varepsilon^{\mathrm{ve}}$ & viscoelastic strain \\
\hline$\varepsilon^{\mathrm{vp}}$ & viscoplastic strain \\
\hline $\mathrm{a}$ & crack length \\
\hline A & cross sectional area \\
\hline$\overline{\mathrm{A}}$ & effective cross-sectional area \\
\hline a & intercept coefficient \\
\hline a & material constant \\
\hline a & regression coefficient \\
\hline A & surface temperature dependent coefficients \\
\hline $\mathrm{A}_{\theta}$ & temperature coefficient \\
\hline $\mathrm{AADT}_{\mathrm{C}}$ & current annual average daily traffic \\
\hline $\mathrm{AADT}_{\mathrm{F}}$ & Future annual average daily traffic \\
\hline$A_{D}$ & surface area with micro defects \\
\hline$a_{t}$ & shift factor \\
\hline $\mathrm{b}$ & the damage exponent of the material \\
\hline $\mathrm{b}$ & ambient temperature coefficient \\
\hline $\mathrm{b}$ & material constant \\
\hline B & material Constants \\
\hline B & surface temperature dependent coefficients \\
\hline $\mathrm{C}$ & compliance \\
\hline & calculated solar radiation coefficient \\
\hline
\end{tabular}




\begin{tabular}{|c|c|}
\hline $\mathrm{C}$ & cloud cover index \\
\hline $\mathrm{C}$ & constant of integration \\
\hline $\mathrm{C}$ & material constant \\
\hline $\mathrm{C}_{1}$ & reference temperature constant \\
\hline $\mathrm{C}_{2}$ & reference temperature constant \\
\hline $\mathrm{Cg}$ & compliance in the glassy regime \\
\hline $\mathrm{Ct}$ & compliance in the rubbery regime \\
\hline $\mathrm{D}$ & damage variable \\
\hline $\mathrm{d}$ & depth \\
\hline $\mathrm{d}$ & depth coefficient, and \\
\hline $\mathrm{D}$ & stiffness matrix \\
\hline $\mathrm{D}_{\mathrm{C}}$ & critical damage variable. \\
\hline DCSE & dissipated creep strain energy \\
\hline $\mathrm{DE}$ & dissipated energy \\
\hline DL & day length \\
\hline e & elasticity \\
\hline e & mechanical deviatoric strains \\
\hline$\dot{\mathrm{e}}$ & rate of deviatoric strains \\
\hline$E^{*}$ & dynamic stiffness modulus of the hot mix asphalt \\
\hline E' & storage Modulus \\
\hline $\mathrm{E}_{0}$ & Young's modulus of undamaged material. \\
\hline $\mathrm{E}_{1}$ & immediate modulus of elasticity \\
\hline $\mathrm{E}_{2}$ & delayed modulus of elasticity \\
\hline$E_{D}$ & Young's modulus of damaged material \\
\hline $\mathrm{EE}$ & elastic energy \\
\hline $\mathrm{E}_{\mathrm{ijkl}}$ & elastic modulus \\
\hline ep & elastoplastic \\
\hline $\mathrm{f}$ & frequency \\
\hline$f$ & thermodynamic force \\
\hline $\mathrm{F}_{0}$ & initial daily flow (base year) \\
\hline $\mathrm{FE}$ & fracture energy \\
\hline FEL & fatigue endurance limit \\
\hline $\mathrm{f}_{\mathrm{R}}$ & reduced frequency at reference temperature \\
\hline G & energy release rate \\
\hline G & shear modulus \\
\hline $\mathrm{G}^{*}$ & complex shear modulus \\
\hline$G$ & long-term shear moduli. \\
\hline $\mathrm{I}_{1}$ & first stress invariant \\
\hline $\mathrm{J}_{2}$ & second stress invariant \\
\hline $\mathrm{J}_{3}$ & third stress invariant \\
\hline$K$ & bulk modulus \\
\hline $\mathrm{k}$ & damage evolution rate \\
\hline $\mathrm{k}$ & material constant \\
\hline $\mathrm{K}_{1}, \mathrm{~K}_{2}$ & material constants \\
\hline$K \infty$ & long-term bulk moduli. \\
\hline $\mathrm{L}$ & distance \\
\hline Lat & latitude of the location \\
\hline $\mathrm{M}_{\mathrm{R}}$ & Resilient modulus \\
\hline $\mathrm{N}$ & the predicted life (repetitions of a) \\
\hline $\mathrm{N}$ & day of the year (with $1^{\text {st }}$ of January $=1$ ) \\
\hline $\mathrm{N}$ & Design life \\
\hline $\mathrm{n}$ & Design life \\
\hline $\mathrm{N}$ & number of load repetition \\
\hline $\mathrm{N}$ & total number of sublavers. \\
\hline
\end{tabular}




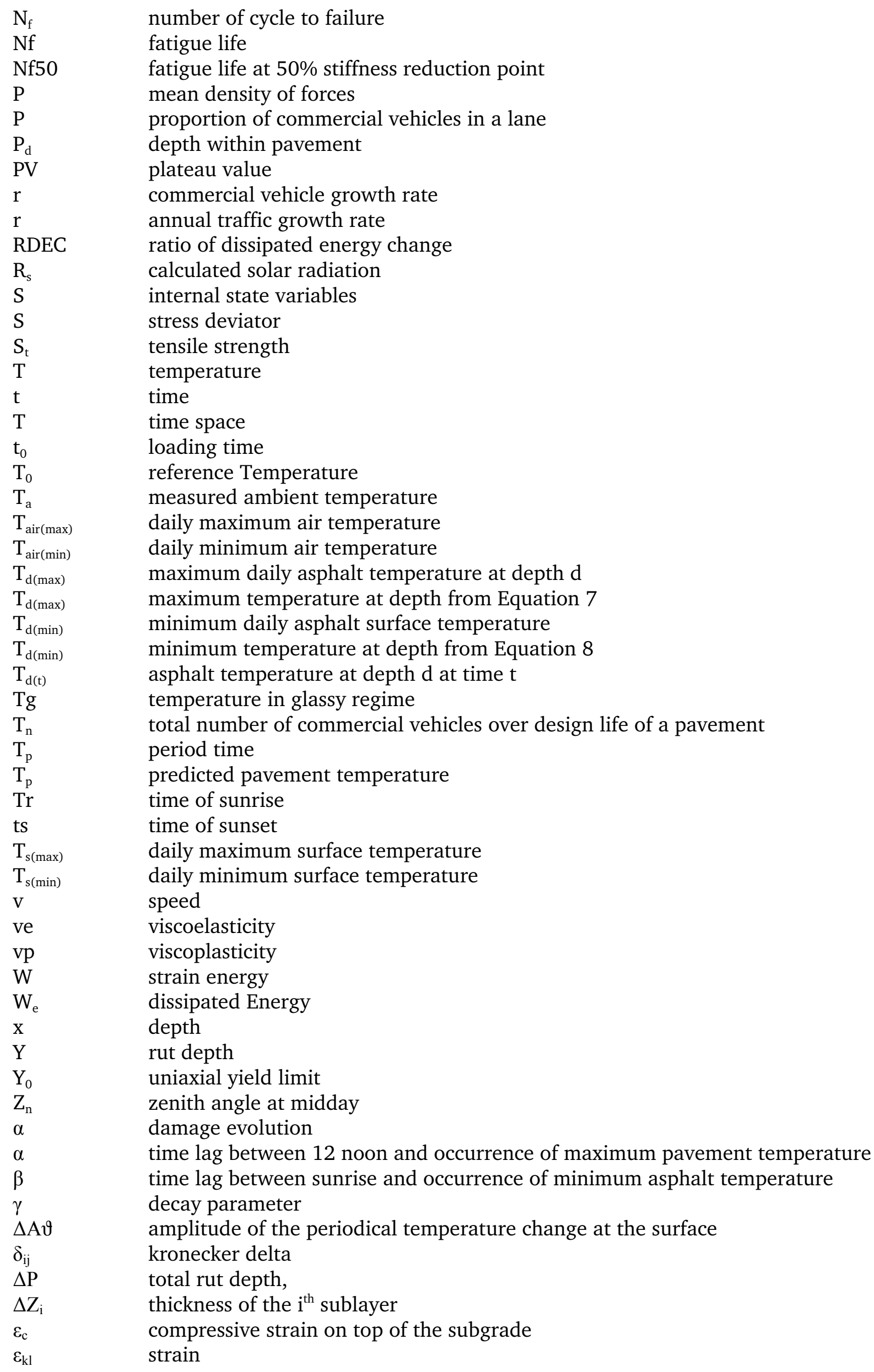




$\begin{array}{ll}\varepsilon_{0} & \text { initial strain } \\ \varepsilon_{\mathrm{t}} & \text { fatigue cracking } \\ \eta & \text { viscous constants } \\ \eta_{1} & \text { immediate Burger's viscoelastic parameter } \\ \eta_{2} & \text { delayed Burger's viscoelastic parameter } \\ \vartheta & \text { temperature function at depth } \\ \vartheta_{\mathrm{FO}} & \text { surface temperature } \\ \vartheta_{\mathrm{M}} & \text { average surface temperature } \\ v & \text { Poisson's ratio }\end{array}$




\section{Lists of Appendices}

Appendix A: Traffic and Vehicular characteristics

A - 1

Appendix B: Material Characteristics

B - 6

Appendix C: Finite Element Analysis Results

C -13

Appendix D: Temperature Variables and Ruttting Model output

D - 23

Appendix E: Existing Rut prediction Models

E - 26

Appendix F: Rut Modelling Program Flow Chart

F -28 



\section{Appendix A: Traffic and Vehicular Characteristics}

\begin{tabular}{|c|c|c|c|}
\hline Class & Type & Description & $\begin{array}{l}\text { Typical } \\
\text { ESALs per } \\
\text { Vehicle }^{2}\end{array}$ \\
\hline 1 & Motorcycles & $\begin{array}{l}\text { All two- or three-wheeled motorized vehicles. Typical vehicles in this } \\
\text { category have saddle type seats and are steered by handle bars rather } \\
\text { than wheels. This category includes motorcycles, motor scooters, } \\
\text { mopeds, motor-powered bicycles, and three-wheel motorcycles. This } \\
\text { vehicle type may be reported at the option of the State. }\end{array}$ & negligible \\
\hline 2 & Passenger Cars & $\begin{array}{l}\text { All sedans, coupes, and station wagons manufactured primarily for } \\
\text { the purpose of carrying passengers and including those passenger cars } \\
\text { pulling recreational or other light trailers. }\end{array}$ & negligible \\
\hline 3 & $\begin{array}{l}\text { Other Two-Axle, } \\
\text { Four-Tire Single Unit } \\
\text { Vehicles }\end{array}$ & $\begin{array}{l}\text { All two-axle, four tire, vehicles, other than passenger cars. Included in } \\
\text { this classification are pickups, panels, vans, and other vehicles such as } \\
\text { campers, motor homes, ambulances, hearses, and carryalls. Other } \\
\text { two-axle, four-tire single unit vehicles pulling recreational or other } \\
\text { light trailers are included in this classification. }\end{array}$ & negligible \\
\hline 4 & Buses & $\begin{array}{l}\text { All vehicles manufactured as traditional passenger-carrying buses } \\
\text { with two axles and six tires or three or more axles. This category } \\
\text { includes only traditional buses (including school buses) functioning as } \\
\text { passenger-carrying vehicles. All two-axle, four-tire single unit } \\
\text { vehicles. Modified buses should be considered to be a truck and be } \\
\text { appropriately classified. }\end{array}$ & 0.57 \\
\hline 5 & $\begin{array}{l}\text { Two-Axle, Six-Tire, } \\
\text { Single Unit Trucks }\end{array}$ & $\begin{array}{l}\text { All vehicles on a single frame including trucks, camping and } \\
\text { recreational vehicles, motor homes, etc., having two axles and dual } \\
\text { rear wheels. }\end{array}$ & 0.26 \\
\hline 6 & $\begin{array}{l}\text { Three-Axle Single Unit } \\
\text { Trucks }\end{array}$ & $\begin{array}{l}\text { All vehicles on a single frame including trucks, camping and } \\
\text { recreational vehicles, motor homes, etc., having three axles. }\end{array}$ & 0.42 \\
\hline 7 & $\begin{array}{l}\text { Four or More Axle } \\
\text { Single Unit Trucks }\end{array}$ & All trucks on a single frame with four or more axles. & 0.42 \\
\hline 8 & $\begin{array}{l}\text { Four or Less Axle Single } \\
\text { Trailer Trucks }\end{array}$ & $\begin{array}{l}\text { All vehicles with four or less axles consisting of two units, one of } \\
\text { which is a tractor or straight truck power unit. }\end{array}$ & 0.30 \\
\hline 9 & $\begin{array}{l}\text { Five-Axle Single Trailer } \\
\text { Trucks }\end{array}$ & $\begin{array}{l}\text { All five-axle vehicles consisting of two units, one of which is a tractor } \\
\text { or straight truck power unit. }\end{array}$ & 1.20 \\
\hline 10 & $\begin{array}{l}\text { Six or More Axle Single } \\
\text { Trailer Trucks }\end{array}$ & $\begin{array}{l}\text { All vehicles with six or more axles consisting of two units, one of } \\
\text { which is a tractor or straight truck power unit. }\end{array}$ & 0.93 \\
\hline 11 & $\begin{array}{l}\text { Five or Less Axle Multi- } \\
\text { Trailer Trucks }\end{array}$ & $\begin{array}{l}\text { All vehicles with five or less axles consisting of three or more units, } \\
\text { one of which is a tractor or straight truck power unit. }\end{array}$ & 0.82 \\
\hline 12 & $\begin{array}{l}\text { Six-Axle Multi-Trailer } \\
\text { Trucks }\end{array}$ & $\begin{array}{l}\text { All six-axle vehicles consisting of three or more units, one of which is } \\
\text { a tractor or straight truck power unit. }\end{array}$ & 1.06 \\
\hline 13 & $\begin{array}{l}\text { Seven or More Axle } \\
\text { Multi-Trailer Trucks }\end{array}$ & $\begin{array}{l}\text { All vehicles with seven or more axles consisting of three or more } \\
\text { units, one of which is a tractor or straight truck power unit. }\end{array}$ & 1.39 \\
\hline
\end{tabular}

Appendix A - 1 ESAL of different vehicle category [49] 


\begin{tabular}{|c|c|c|c|c|c|}
\hline $\begin{array}{l}\text { Truck } \\
\text { Num. }\end{array}$ & Truck Configuration & Configuration Name & $\begin{array}{l}\text { GCVW } \\
\text { (kips) }\end{array}$ & $\begin{array}{c}\text { Axle Loads } \\
\text { (kips) }\end{array}$ & $\begin{array}{c}\text { Wheelbases } \\
\text { (feet) }\end{array}$ \\
\hline $1-2$ & & 2 Axle Straight Truck & 32 & $12 / 20$ & 15 \\
\hline $3-4$ & & 3 Axle Straight Truck & 46 & $12 / 34$ & 18 \\
\hline $5-8$ & & 3 Axle Refuse Hauler & 64 & $20 / 44$ & 17.5 \\
\hline $9-12$ & & 4 Axle Concrete Mixer & 68 & $18 / 38 / 12$ & $20 / 12$ \\
\hline 13 & & 3 Axle Tractor-Semitrailer & 52 & $12 / 20 / 20$ & $10 / 36$ \\
\hline 14-15 & 8 & 4 Axle Tractor-Semitrailer & 66 & $12 / 20 / 34$ & $12 / 36$ \\
\hline $16-20$ & & 5 Axle Tractor-Semitrailer & 80 & $12 / 34 / 34$ & $12 / 36$ \\
\hline 21 & 8 & 5 Axle Tractor-Semitrailer & 80 & $14 / 33 / 33$ & $10 / 36$ \\
\hline 22 & $8 \longdiv { 8 0 }$ & 5 Axle Tanker & 80 & $12 / 34 / 34$ & $12 / 36$ \\
\hline $23-24$ & $8 \longdiv { 0 0 }$ & 6 Axle Tanker & 85 & $12 / 34 / 39$ & $12 / 38$ \\
\hline 25 & & 5 Axle Doubles & 80 & $10 / 18 / 17 / 18 / 17$ & $10 / 22 / 22$ \\
\hline 26 & & 5 Axle Doubles & 80 & $10 / 20 / 15 / 20 / 15$ & $10 / 22 / 22$ \\
\hline 27 & & 7 Axle Doubles & 120 & $12 / 34 / 34 / 20 / 20$ & $12 / 38 / 22$ \\
\hline 28 & 800 & 9 Axle Doubles & 140 & $12 / 32 / 32 / 32 / 32$ & $12 / 38 / 38$ \\
\hline 29 & 80 & Tumer Doubles & 114 & $10 / 26 / 26 / 26 / 26$ & $12 / 22 / 22$ \\
\hline
\end{tabular}

* Wheelbases to tandem centers. Tandem spreads set at 52 inches.

Appendix A - 2 Truck matrix sizes and weights [Gillespie 1993] 


\begin{tabular}{|c|c|c|c|c|c|c|}
\hline & \multicolumn{2}{|c|}{ Lorries } & \multicolumn{2}{|c|}{ Road train } & \multirow{2}{*}{$\begin{array}{c}\text { Articulated } \\
\text { vehicles } \\
5 \text { axles and } \\
\text { more }\end{array}$} & \\
\hline & 2 axles & 3 axles & 4 axles & $\begin{array}{c}5 \text { axles and } \\
\text { more }\end{array}$ & & \\
\hline $\mathrm{BE}$ & 19 & 26 & 39 & 44 & 44 & $\mathrm{BE}$ \\
\hline BG & 16 & 26 & 36 & 40 & 40 & $B G$ \\
\hline $\mathrm{CZ}$ & 18 & $25 / 26$ & 36 & 42 & 42 & $\mathrm{CZ}$ \\
\hline DK & $18 / 19$ & $24 / 26$ & 38 & $44 / 40$ & $40 / 48$ & DK \\
\hline $\mathrm{DE}$ & 18 & 26 & 36 & 40 & 40 & $\mathrm{DE}$ \\
\hline $\mathrm{EE}$ & 18 & 26 & 36 & 40 & 40 & $\mathrm{EE}$ \\
\hline IE & 17 & 26 & 35 & 40 & 40 & IE \\
\hline EL & 18 & 26 & 36 & 40 & 40 & EL \\
\hline ES & 18 & 26 & 36 & 40 & 40 & ES \\
\hline FR & 19 & 26 & 38 & 40 & 40 & FR \\
\hline IT & 18 & 26 & 40 & 44 & 44 & IT \\
\hline $\mathrm{CY}$ & & & & & & $\mathrm{CY}$ \\
\hline LV & 18 & 25 & 36 & 40 & 40 & LV \\
\hline LT & 18 & 26 & 36 & 40 & 40 & LT \\
\hline LU & 19 & 26 & & 44 & 44 & LU \\
\hline $\mathrm{HU}$ & 20 & 24 & 36 & 40 & 40 & $\mathrm{HU}$ \\
\hline MT & & & 40 & 40 & 40 & MT \\
\hline NL & 21.5 & 33 & 40 & 50 & 50 & NL \\
\hline AT & 18 & 25 & 36 & 38 & 38 & AT \\
\hline PL & 19.5 & 29.5 & 37 & 40 & 40 & PL \\
\hline PT & 19 & 26 & 38 & 40 & 40 & PT \\
\hline Ro & 18 & 24 & 34 & 40 & 40 & Ro \\
\hline $\mathbf{s} \mathbf{I}$ & 18 & 25 & & 40 & 40 & $\mathbf{S I}$ \\
\hline SK & 18 & 26 & 40 & 40 & 40 & SK \\
\hline $\mathrm{FI}$ & 18 & 26 & 38 & 60 & 48 & $\mathrm{FI}$ \\
\hline SE & 18 & 26 & & 60 & 60 & SE \\
\hline UK & 18 & 26 & 36 & 40 & 40 & UK \\
\hline HR & & & 40 & 40 & 40 & HR \\
\hline MK & & & 40 & 40 & 40 & MK \\
\hline TR & 18 & 25 & 36 & 40 & 40 & TR \\
\hline IS & 18 & 26 & 37 & 40 & 44 & IS \\
\hline NO & & 26 & & 50 & 47 & NO \\
\hline $\mathrm{CH}$ & 18 & 25 & 34 & 34 & 34 & $\mathrm{CH}$ \\
\hline LI & 18 & 26 & 36 & 40 & 40 & LI \\
\hline
\end{tabular}

Source : National sources, International Transport Forum

Notes :

An articulated vehicle consists of a road tractor coupled to a semi-trailer. A road train is a goods road motor vehicle coupled to one or more trailers.

DK: national / international

Many countries allow higher weight limits in certain circumstances: wheelbase, rear-axle spacing, double tyres, type of engine, type of road, type of load (containers).

Appendix A - 3 Maximum gross vehicles weight in EU countries [Capros et al. 2008] 
Freight Transport

1000 mio tonne-kilometres

\begin{tabular}{|c|c|c|c|c|c|c|c|}
\hline & Road & Rail & $\begin{array}{c}\text { Inland } \\
\text { Waterways }\end{array}$ & Oil Pipelines & Sea & Air & Total \\
\hline 2006 & 1888 & 435 & 138 & 135 & 1545 & 3.0 & 4143 \\
\hline 2005 & 1800 & 413 & 138 & 136 & 1530 & 2.9 & 4020 \\
\hline 2004 & 1747 & 413 & 136 & 131 & 1488 & 2.8 & 3918 \\
\hline 2003 & 1625 & 391 & 123 & 130 & 1445 & 2.6 & 3717 \\
\hline 2002 & 1606 & 382 & 132 & 128 & 1417 & 2.6 & 3668 \\
\hline 2001 & 1556 & 385 & 132 & 132 & 1400 & 2.7 & 3607 \\
\hline 2000 & 1519 & 401 & 133 & 126 & 1348 & 2.7 & 3529 \\
\hline 1999 & 1470 & 383 & 127 & 124 & 1288 & 2.5 & 3394 \\
\hline 1998 & 1414 & 392 & 130 & 125 & 1243 & 2.4 & 3307 \\
\hline 1997 & 1352 & 409 & 126 & 118 & 1205 & 2.3 & 3213 \\
\hline 1996 & 1303 & 392 & 118 & 119 & 1162 & 2.1 & 3096 \\
\hline 1995 & 1289 & 386 & 121 & 115 & 1150 & 2.0 & 3062 \\
\hline $\begin{array}{l}1995 \\
2006\end{array}$ & $+46.5 \%$ & $+12.6 \%$ & $+14.5 \%$ & $+17.2 \%$ & $+34.3 \%$ & $+50.0 \%$ & $+35.3 \%$ \\
\hline $\begin{array}{l}\text { per } \\
\text { year }\end{array}$ & $+3.5 \%$ & $+1.1 \%$ & $+1.2 \%$ & $+1.5 \%$ & $+2.7 \%$ & $+3.8 \%$ & $+2.8 \%$ \\
\hline $\begin{array}{r}2005 \\
2006\end{array}$ & $+4.9 \%$ & $+5.2 \%$ & $+0.0 \%$ & $-0.7 \%$ & $+1.0 \%$ & $+3.4 \%$ & $+3.1 \%$ \\
\hline
\end{tabular}

Appendix A - 4 Freight transport (EU) [Capros et al. 2008]

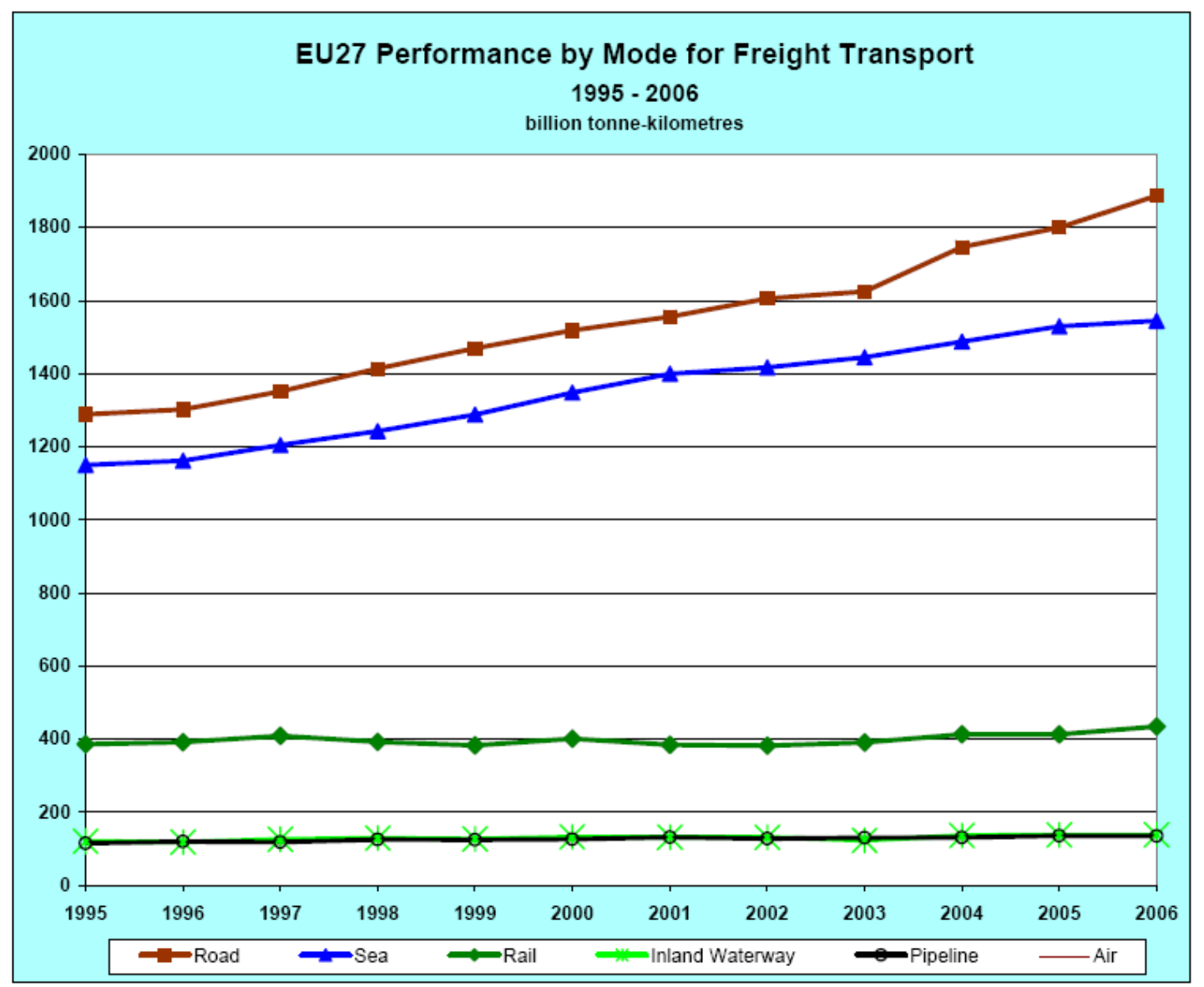

Source: tables $3.2 .4 \mathrm{c}$ to 3.2 .7 , estimates

Appendix A - 5 Freight transport growth (EU) [Capros et al. 2008] 


\begin{tabular}{|c|c|c|c|c|c|}
\hline & \multicolumn{3}{|c|}{ Speed limit, cars (in general), $\mathrm{km} / \mathrm{h}$ : } & \multirow{2}{*}{\multicolumn{2}{|c|}{$\begin{array}{c}\text { Blood alcohol } \\
\text { limit, grams of } \\
\begin{array}{c}\text { alcohol in } 1 \text { litre of } \\
\text { blood }\end{array}\end{array}$}} \\
\hline & Built-up areas & $\begin{array}{l}\text { Outside built-up } \\
\text { areas }\end{array}$ & Motorways & & \\
\hline $\mathrm{BE}$ & 50 & $90-120$ & 120 & 0.5 & $\mathrm{BE}$ \\
\hline BG & 50 & 90 & 120 & 0.5 & BG \\
\hline$C Z$ & 50 & $90-130$ & 130 & 0 & $\mathrm{CZ}$ \\
\hline DK & 50 & 80 & $110-130$ & 0.5 & DK \\
\hline $\mathrm{DE}$ & 50 & 100 & (130) & 0.5 & $\mathrm{DE}$ \\
\hline $\mathrm{EE}$ & 50 & $90-110$ & 110 & 0 & $\mathrm{EE}$ \\
\hline $\mathrm{IE}$ & 50 & $80-100$ & 120 & 0.8 & $\mathrm{IE}$ \\
\hline EL & 50 & $90-110$ & 120 & 0.5 & EL \\
\hline ES & 50 & $90-100$ & 120 & 0.5 & ES \\
\hline FR & 50 & $80-110$ & $110-130$ & 0.5 & FR \\
\hline IT & 50 & $90-110$ & $130-150$ & 0.5 & IT \\
\hline $\mathrm{CY}$ & 50 & 80 & 100 & 0.9 & $\mathrm{CY}$ \\
\hline LV & 50 & $90-100$ & 110 & 0.5 & LV \\
\hline LT & 50 & $90-100$ & $110-130$ & 0.4 & LT \\
\hline LU & 50 & 90 & 130 & 0.5 & LU \\
\hline HU & 50 & $90-110$ & 130 & 0 & $\mathrm{HU}$ \\
\hline MT & 50 & $60-80$ & - & 0.8 & MT \\
\hline NL & 50 & $80-100$ & $100-120$ & 0.5 & NL \\
\hline AT & 50 & 100 & 130 & 0.5 & AT \\
\hline PL & $50-60$ & $90-110$ & 130 & 0.2 & PL \\
\hline PT & 50 & $90-100$ & 120 & 0.5 & PT \\
\hline RO & 50 & $90-100$ & 130 & 0 & RO \\
\hline SI & 50 & $90-100$ & 130 & 0.5 & $\mathbf{S I}$ \\
\hline SK & 60 & 90 & 130 & 0 & SK \\
\hline $\mathrm{FI}$ & 50 & $80-100$ & $100-120$ & 0.5 & $\mathrm{FI}$ \\
\hline SE & $30-50$ & $70-110$ & $90-110$ & 0.2 & SE \\
\hline UK & 48 & $96-112$ & 112 & 0.8 & UK \\
\hline HR & 50 & $90-100$ & 130 & 0 & HR \\
\hline MK & 60 & $80-100$ & 120 & 0.5 & MK \\
\hline TR & 50 & 90 & 130 & 0.5 & TR \\
\hline IS & 50 & $80-90$ & - & 0.5 & IS \\
\hline NO & 50 & 80 & $90-100$ & 0.2 & NO \\
\hline $\mathrm{CH}$ & 50 & 80 & 120 & 0.5 & $\mathrm{CH}$ \\
\hline
\end{tabular}

Source: National sources, International Transport Forum

Notes :

UK, IE, CY and MT drive on the left hand side of the road, the other Member States drive on the right hand side (Sweden since 3 September 1967). Signs in UK are in miles per hour.

The higher figure shown in the "outside built-up areas" column generally refers to the speed limit on dual-carriageways that are not motorways.

Speed limits:

DE: Motorways: No general speed limit, recommended speed limit is $130 \mathrm{~km} / \mathrm{h}$ (more than half the network has a speed limit of $120 \mathrm{~km} / \mathrm{h}$ or less).

FR: Dual carriageways $110 \mathrm{~km} / \mathrm{h}$. If the road is wet : motorways $110 \mathrm{~km} / \mathrm{h}$, dual carriageways $90 \mathrm{~km} / \mathrm{h}$, other roads outside built-up areas $80 \mathrm{~km} / \mathrm{h}$.

AT, IT: $150 \mathrm{~km} / \mathrm{h}$ on certain $2 \times 3$ lane motorways.

Fl: in winter $100 \mathrm{~km} / \mathrm{h}$ on motorways, $80 \mathrm{~km} / \mathrm{h}$ on other roads.

PL: Built-up areas: $50 \mathrm{~km} / \mathrm{h}$ from $05 \mathrm{~h} 00$ to $23 \mathrm{~h} 00,60 \mathrm{~km} / \mathrm{h}$ from $23 \mathrm{~h} 00$ to $05 \mathrm{~h} 00$.

Appendix A - 6 Speed and Alcohol limit (EU) [Capros et al. 2008] 


\section{Appendix B: Material Characteristics}

\begin{tabular}{lccccc}
\hline $\begin{array}{l}\text { Mixture } \\
\text { denotation }\end{array}$ & $\begin{array}{c}E_{1} \\
(\mathrm{GPa})\end{array}$ & $\begin{array}{c}\eta_{1} \\
(\mathrm{GPas})\end{array}$ & $\begin{array}{c}E_{2} \\
(\mathrm{GPa})\end{array}$ & $\begin{array}{c}\eta_{2} \\
(\mathrm{GPas})\end{array}$ & $\begin{array}{c}\Delta_{i}^{p} \\
(\%)\end{array}$ \\
\hline mw1 & 0.162 & 1480 & 0.178 & 10.0 & 5.57 \\
$\mathrm{mb1}$ & 0.166 & 1510 & 0.202 & 13.0 & 6.86 \\
$\mathrm{mbcl}$ & 0.309 & 3250 & 0.528 & 27.0 & 4.98 \\
\hline
\end{tabular}

Table B1 . Rheological Parameters and approximation Errors for Selected Mixes (Third Cycle)

\begin{tabular}{lcccccc}
\hline $\begin{array}{l}\text { Mixture } \\
\text { denotation }\end{array}$ & $\begin{array}{c}E_{1} \\
(\mathrm{GPa})\end{array}$ & $\begin{array}{c}\eta_{1} \\
(\mathrm{GPas})\end{array}$ & $\begin{array}{c}E_{2} \\
(\mathrm{GPa})\end{array}$ & $\begin{array}{c}\eta_{2} \\
(\mathrm{GPas})\end{array}$ & $\begin{array}{c}\text { Bitumen } \\
\text { content } \%\end{array}$ & $\begin{array}{c}\text { Voids } \\
(\%)\end{array}$ \\
\hline $\begin{array}{l}\text { Wearing course } \\
\text { Binder course }\end{array}$ & $0.068-0.235$ & $495-2,600$ & $0.033-0.411$ & $3.0-21.0$ & $3.4-6.3$ & $1.8-5.6$ \\
Base course & $0.068-0.315$ & $340-3,350$ & $0.0971-0.463$ & $4.0-25.0$ & $3.5-6.7$ & $3.8-8.0$ \\
\hline
\end{tabular}

Table B2. Variation of Rheological Parameters of Mixes Designed for Different Courses

\begin{tabular}{lccccc}
\hline $\begin{array}{l}\text { Course } \\
\text { (mixture) }\end{array}$ & $\begin{array}{c}\text { Temperature } \\
\left({ }^{\circ} \mathrm{C}\right)\end{array}$ & $\begin{array}{c}E_{1} \\
(\mathrm{GPa})\end{array}$ & $\begin{array}{c}\eta_{1} \\
(\mathrm{GPas})\end{array}$ & $\begin{array}{c}E_{2} \\
(\mathrm{GPa})\end{array}$ & $\begin{array}{c}\eta_{2} \\
(\mathrm{GPas})\end{array}$ \\
\hline $\begin{array}{l}\text { Wearing course } \\
(\mathrm{mw} 1)\end{array}$ & 42 & 0.192 & 2,490 & 0.197 & 7.74 \\
$\begin{array}{l}\text { Binder course } \\
(\mathrm{mb} 1)\end{array}$ & 35 & 0.223 & 2,350 & 0.225 & 9.51 \\
$\begin{array}{l}\text { Base course } \\
(\mathrm{mbc} 1)\end{array}$ & 25 & 0.527 & 2,490 & 0.965 & 13.3 \\
\hline
\end{tabular}

Table B3. Rheological Parameters for Pavement Courses (Roadway Surface Temperature: $45^{\circ} \mathrm{C}$ )

Appendix B - 1 Viscoelastic material Parameters [Szydlo and Mackiewicz 2005] 


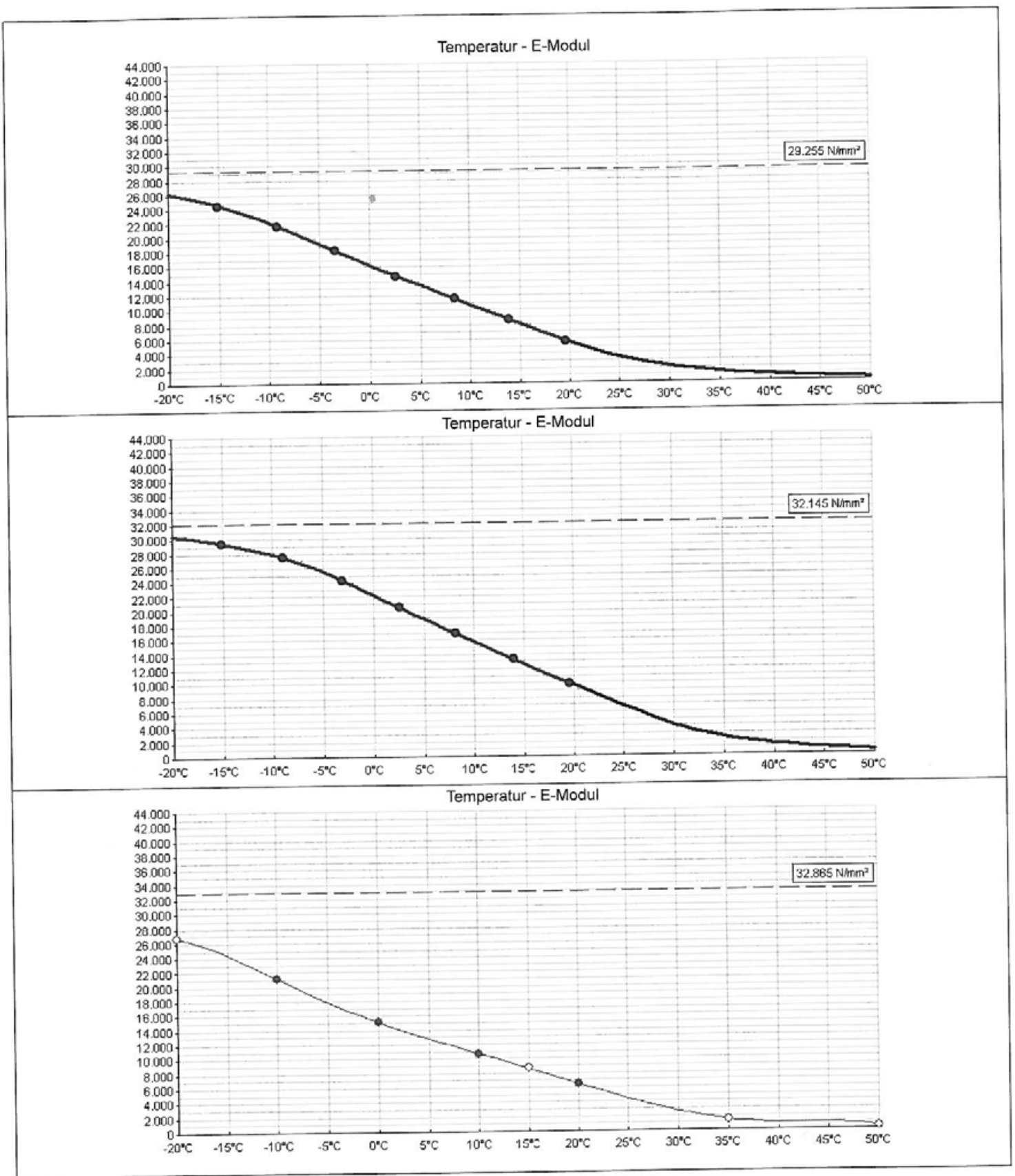

Bild 98: Elastizitätsmodulkennlinien der Kalibriermaterialien für Asphaltdeckschicht (oben), Asphaltbinderschicht (Mitte) und Asphalttragschicht (unten) - Screenshots aus PADESTO (Quelle: [28])

Appendix B - 2 Variation of elastic modulus with respect to temperature [Wellner Kayser 2008] 
Tabelle 5-9: Erforderliche Mindest-Schichtmoduln der Gesteinskorngemische zur Einhaltung der geforderten Verformungsmoduin (gemäß RStO 01, Tafel 1)

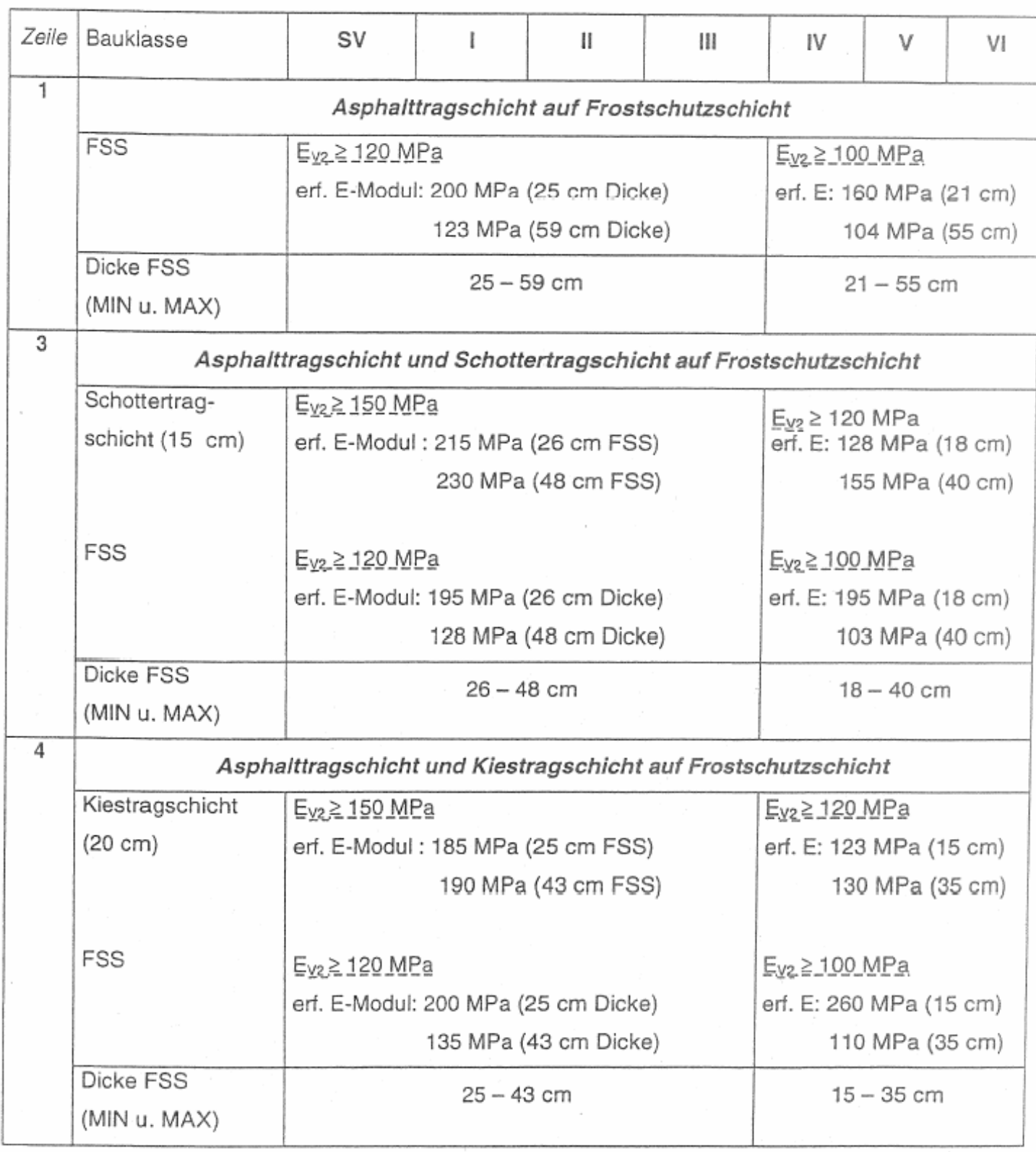

Appendix B - 3 Recommended materials Parameters for different traffic class as per German Standard [79] 
Tabelle 5-8: E-Moduln des Modellasphaltes in unterschiedlichen Tiefen bei verschiedenen Oberflächentemperaturen

\begin{tabular}{|c|c|c|c|c|c|}
\hline Nr. & Tiefe & \multicolumn{5}{|c|}{ E-Modul der Asphaltschicht [MPa] bei Oberflächentemperatur } \\
\hline$[-]$ & {$[\mathrm{cm}]$} & $-7,5^{\circ} \mathrm{C}$ & $7,5^{\circ} \mathrm{C}$ & $27,5^{\circ} \mathrm{C}$ & $47,5^{\circ} \mathrm{C}$ \\
\hline 1 & $0-2$ & 15136 & 10103 & 3815 & 863 \\
\hline 2 & $2-4$ & 14929 & 10103 & 3962 & 1149 \\
\hline 3 & $4-6$ & 17716 & 10103 & 4114 & 1435 \\
\hline 4 & $6-8$ & 14529 & 10066 & 4242 & 1781 \\
\hline 5 & $8-10$ & 14338 & 10066 & 4373 & 2192 \\
\hline 6 & $10-12$ & 14177 & 10066 & 4480 & 2496 \\
\hline 7 & $12-14$ & 14013 & 10066 & 4588 & 2688 \\
\hline 8 & $14-16$ & 13880 & 10066 & 4670 & 2880 \\
\hline 9 & $16-18$ & 13779 & 10066 & 4753 & 3072 \\
\hline 10 & $18-20$ & 13644 & 10029 & 4837 & 3312 \\
\hline 11 & $20-22$ & 13576 & 10029 & 4893 & 3532 \\
\hline 12 & $22-24$ & 13474 & 10029 & 4949 & 3685 \\
\hline 13 & $24-26$ & 13405 & 10029 & 5006 & 3838 \\
\hline 14 & $26-28$ & 13336 & 10029 & 5064 & 3991 \\
\hline 15 & $28-30$ & 13267 & 10029 & 5122 & 4144 \\
\hline 16 & $30-32$ & 13197 & 10029 & 5180 & 4297 \\
\hline 17 & $32-34$ & 13127 & 10029 & 5209 & 4450 \\
\hline
\end{tabular}

Appendix B - 4 Variations of elastic modulus with respect to depth at deferent surface temperature [79]

Tabelle 5-1: Schichtmoduin und Querdehnzahlen für ToB und Böden

\begin{tabular}{|l|c|c|}
\hline \multicolumn{1}{|c|}{ Schichtart } & $\begin{array}{c}\text { Schichtmodul } \\
{\left[\mathrm{MN} / \mathrm{m}^{2}\right]}\end{array}$ & $\begin{array}{c}\text { Querdehnzahl } \\
\mu[-]\end{array}$ \\
\hline $\begin{array}{l}\text { FSS nach ZTV T-StB: } \\
\text { Kiese und Kies-Sand-Gemische der Gruppe: GE } \\
\text { Sande und Sand-Kies-Gemische der Gruppe: SE }\end{array}$ & 0,5 bis 100 \\
\hline $\begin{array}{l}\text { FSS nach ZTV T-StB: } \\
\text { Kiese und Kies-Sand-Gemische der Gruppe: GI, GW } \\
\text { Sande und Sand-Kies-Gemische der Gruppe: SI, SW }\end{array}$ & 100 bis 150 & 0,5 \\
\hline $\begin{array}{l}\text { FSS nach ZTV T-StB, Gemische aus gebrochenen } \\
\text { Gesteinskörnungen }\end{array}$ & 150 bis 200 & 0,5 \\
\hline Kiestragschicht nach ZTV T-StB & 150 bis 250 & 0,5 \\
\hline Schottertragschicht nach ZTV T-StB & 250 bis 400 & 0,5 \\
\hline Böden (z.B. TL, SU*, SU) & 10 bis 80 & 0,5 \\
\hline
\end{tabular}

Appendix B - 5 Layer modulus and poisson's for base (without binding material) and subgrade layers [79] 

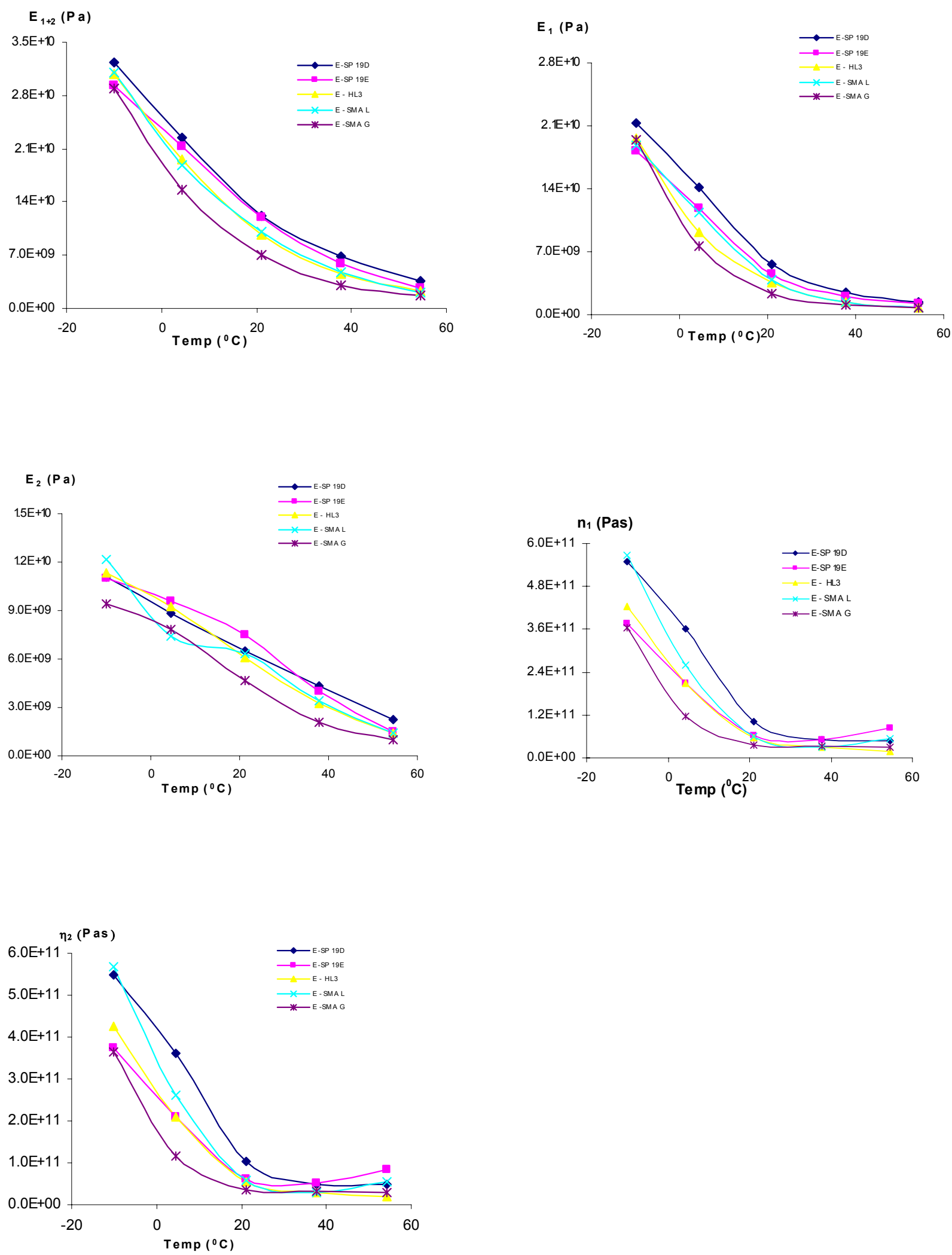

Appendix B - 6 Mtl0-Regression analysis of material characteristics (from Uzarowski 2006) 

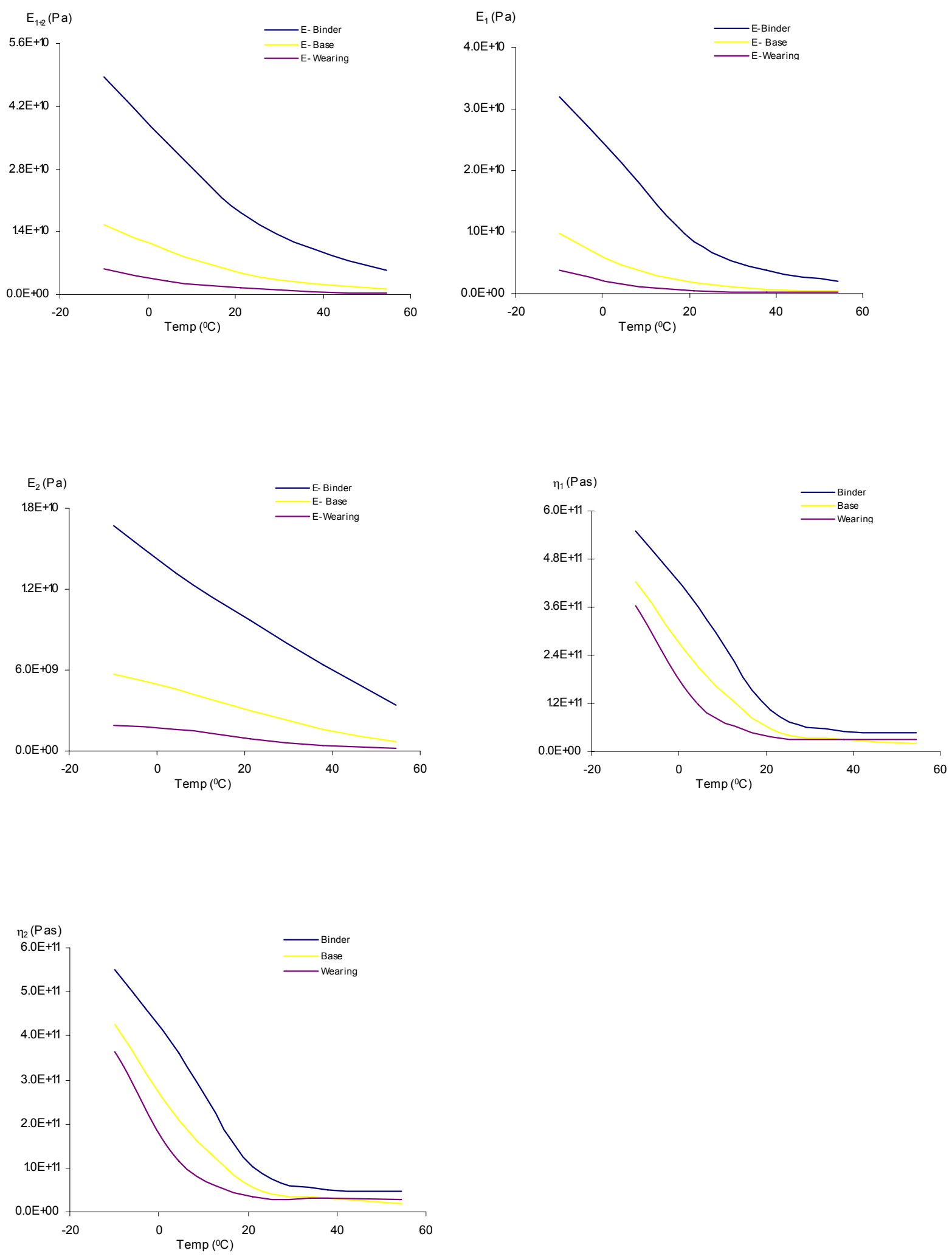

Appendix B - 7 Mtl1-Material characteristics with respect to temperature 

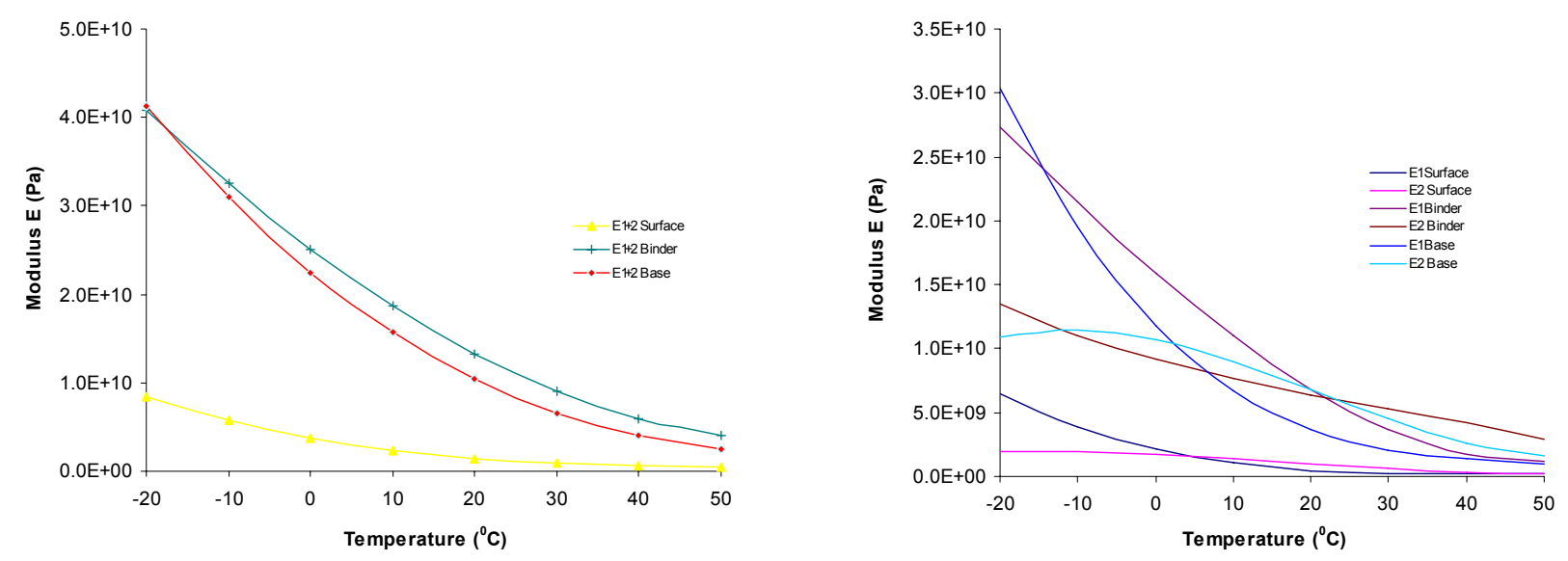

MtIE2- Variations of elastic modulus with respect to temperature
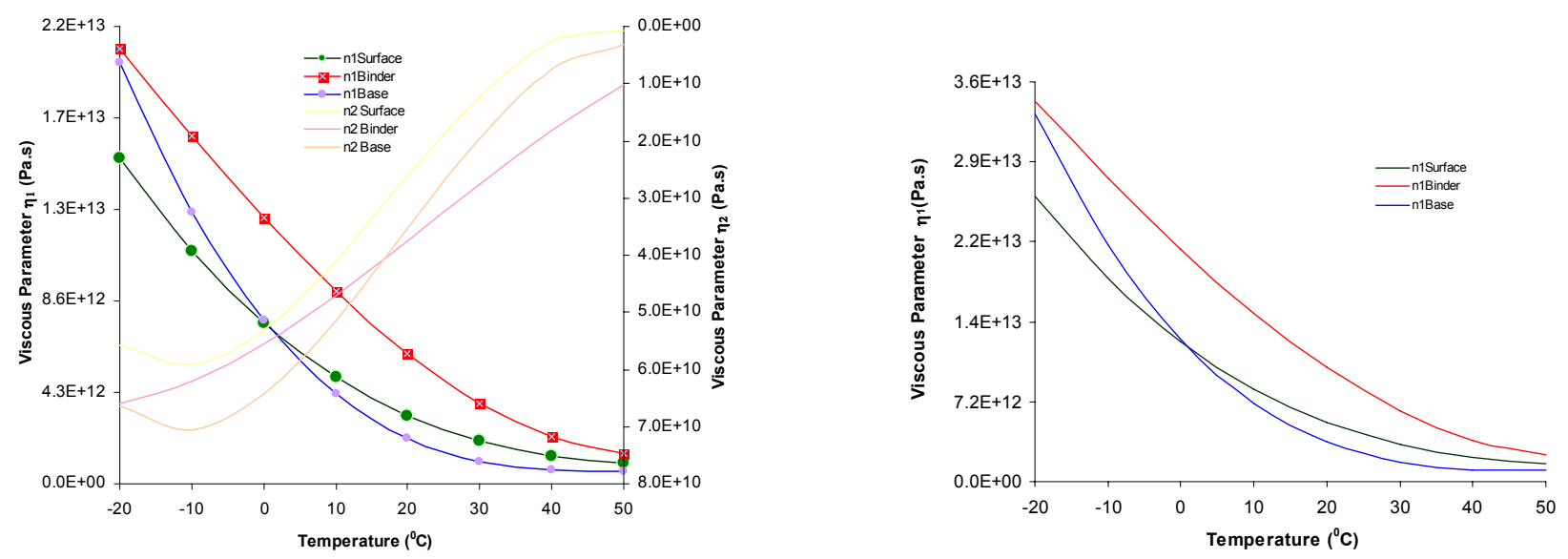

MtVis30

$\mathrm{MtViN}_{1} 50$

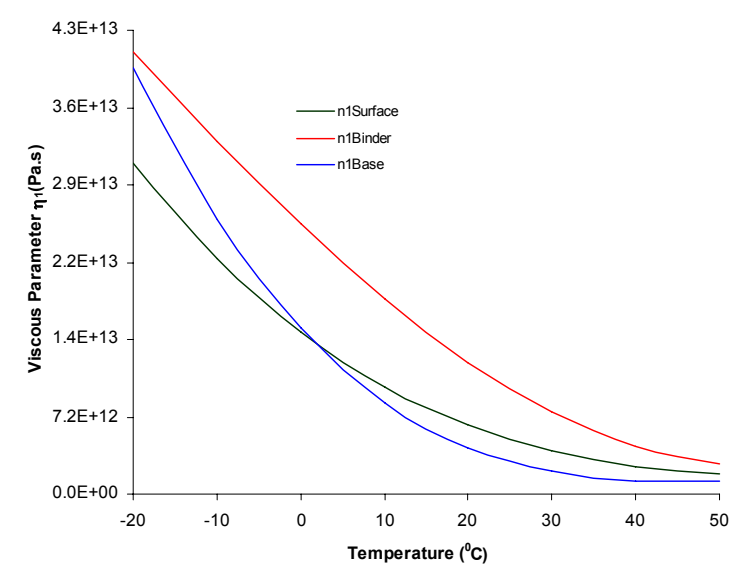

$\operatorname{MtViN}_{1} 60$

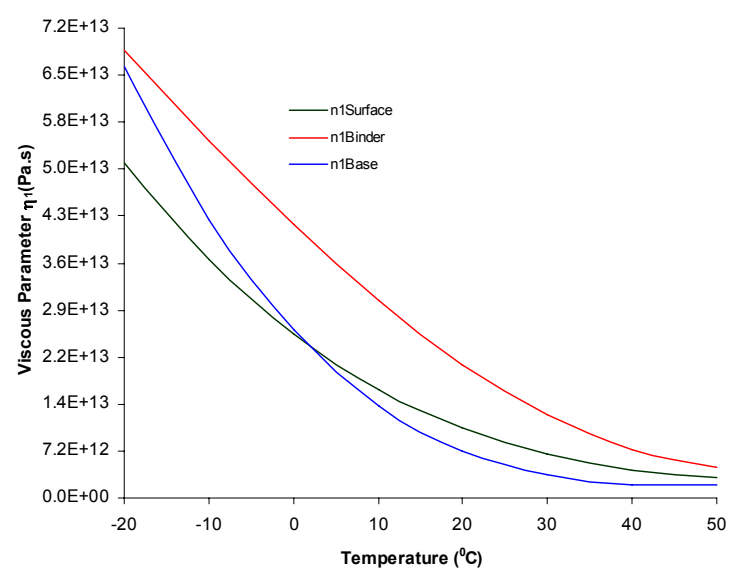

$\operatorname{MtViN}_{1} 100$

Appendix B - 8 Variation of material characteristics with respect to temperature 


\section{Appendix C: Finite Element Analysis Results}

S, Max. In-Plane Principal

s, Min. In-Plane Principal

s, Out-of-Plane Principal
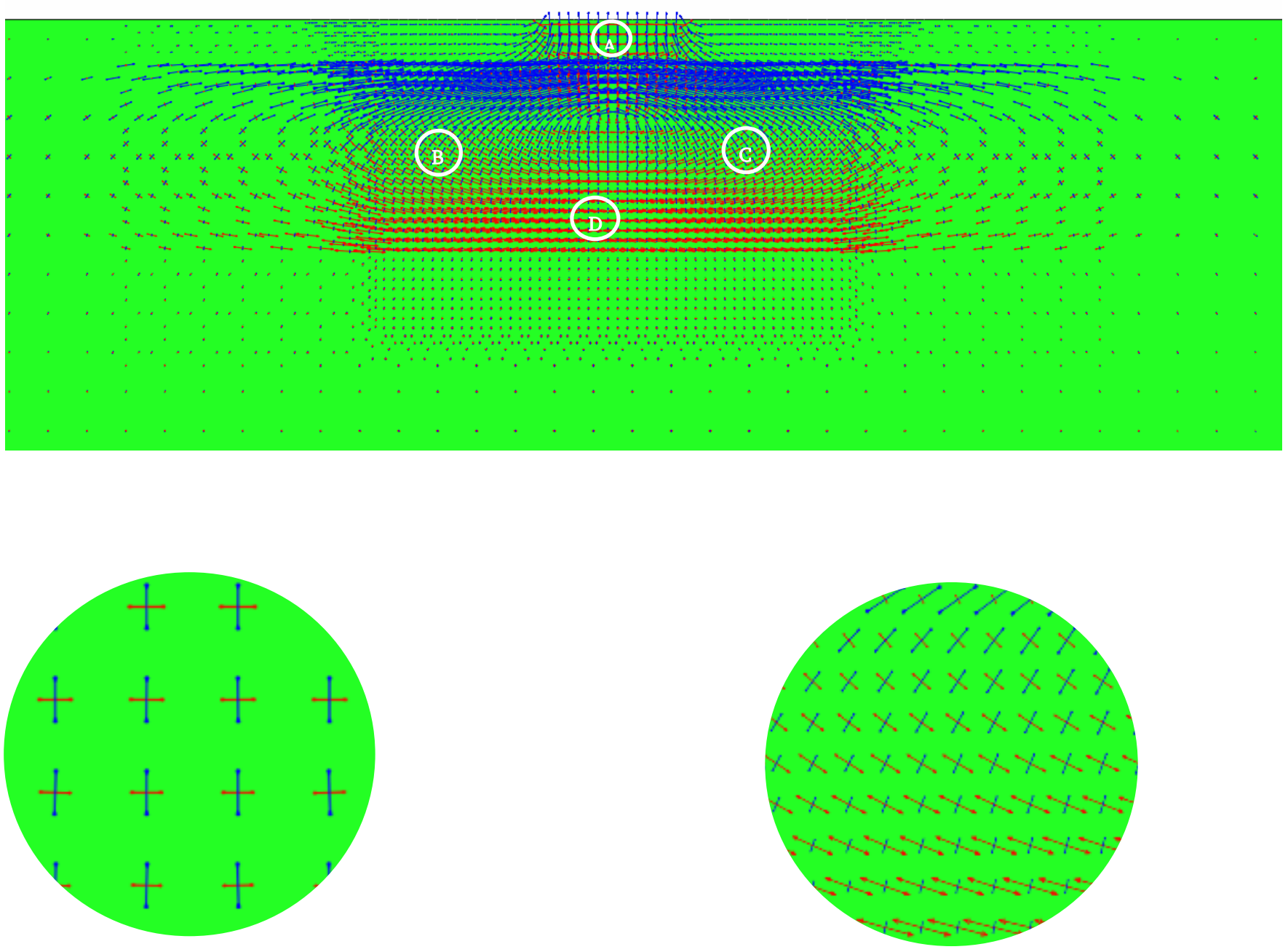

Section A

Section B
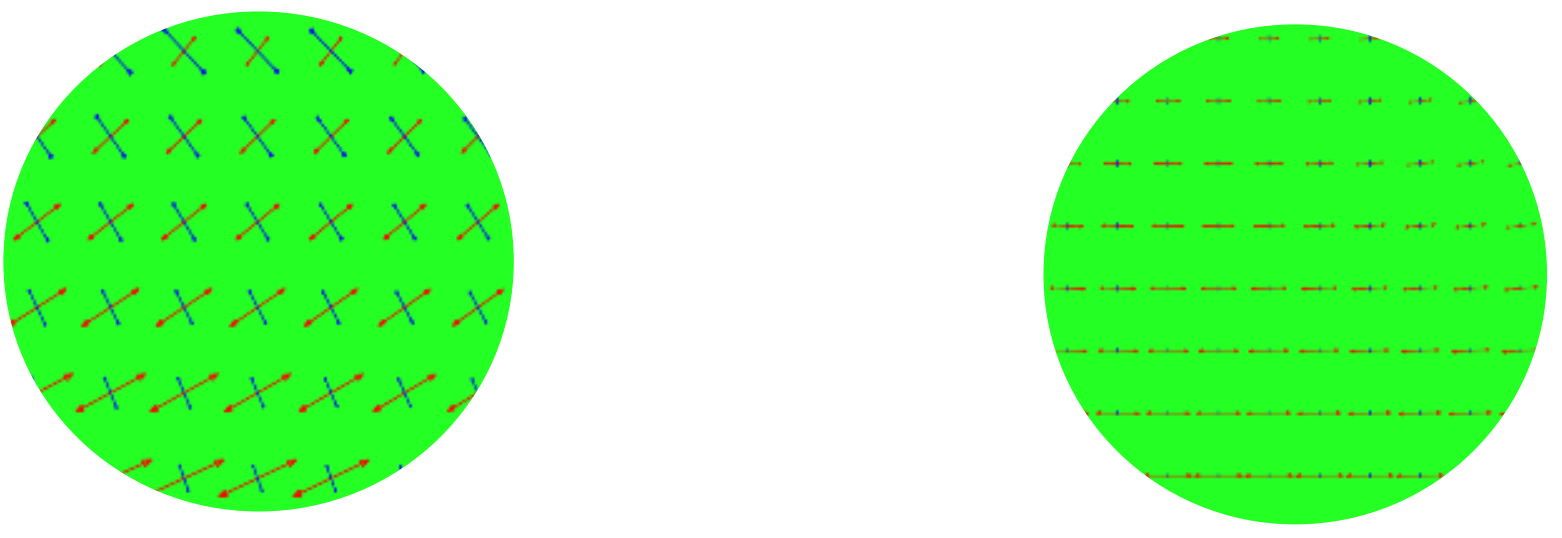

Section C

Section D

Appendix C - 1 Orientation of principal stresses 

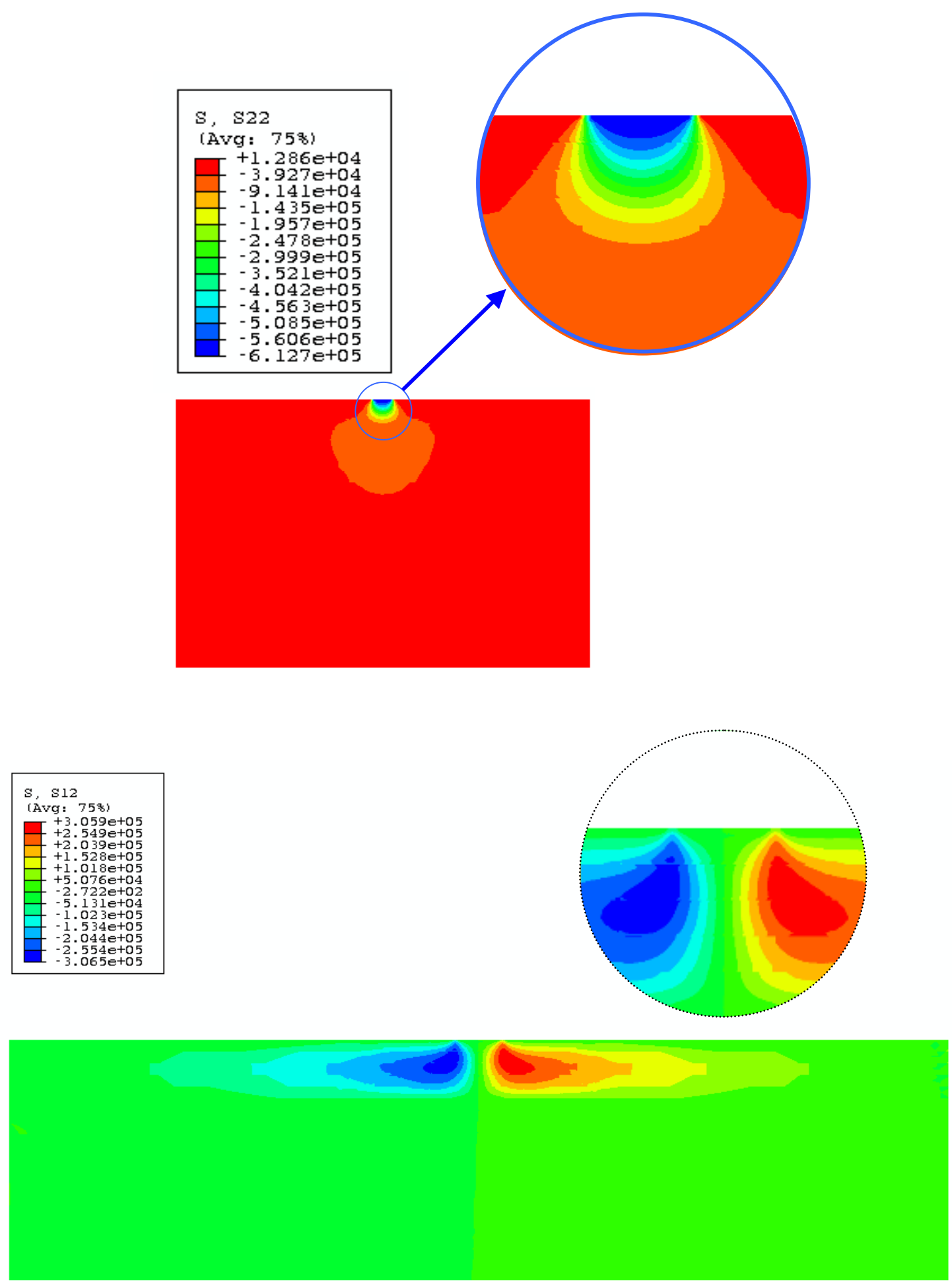

Appendix C - 2 Vertical and shear stresses with single wheel load 

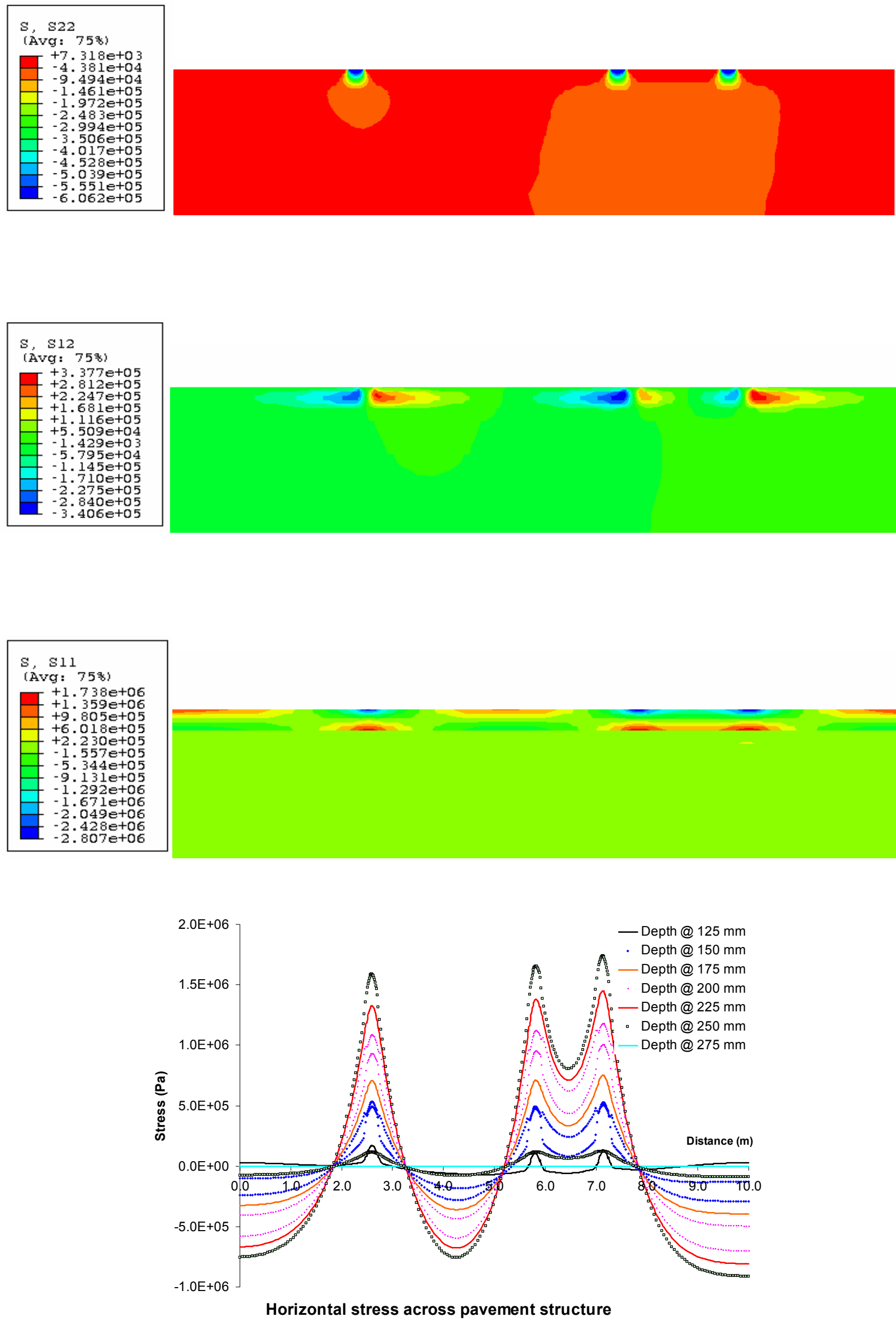

Appendix C - 3 Vertical, shear and lateral stresses with multiple wheel load 

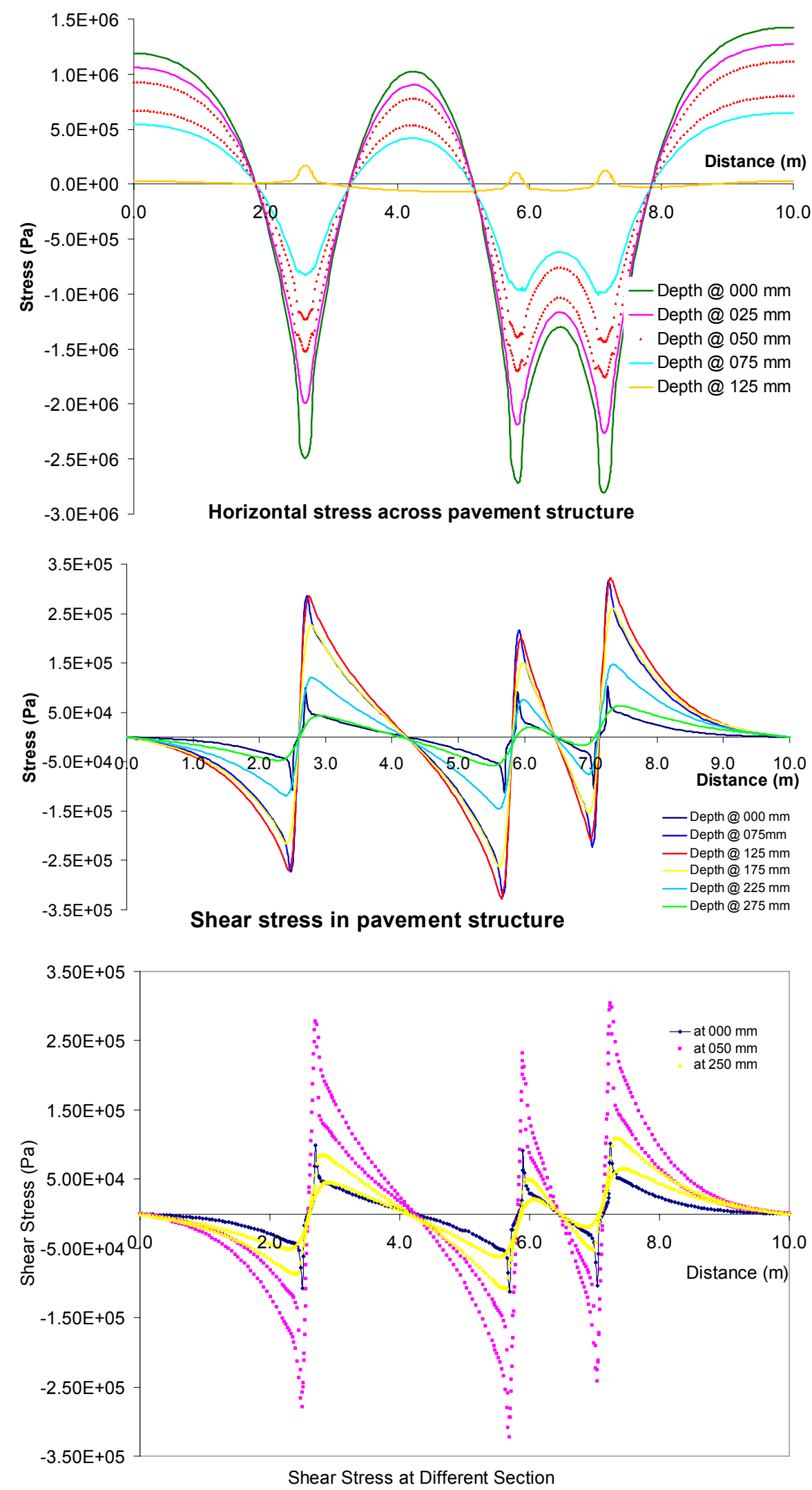

Appendix C - 4 Vertical, shear and lateral stresses with multiple wheel load (continued) 


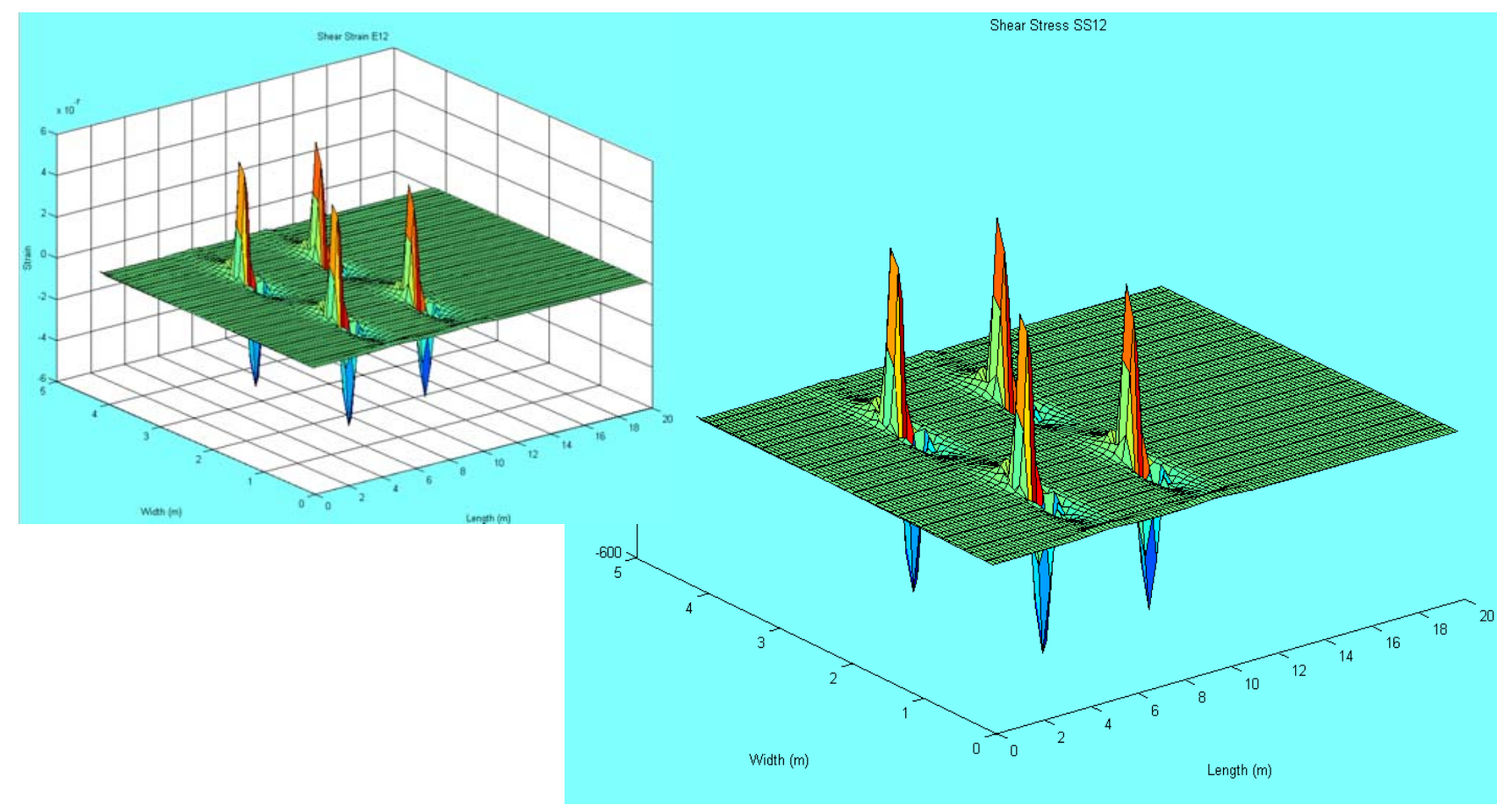

a. shear strain

b. shear stress

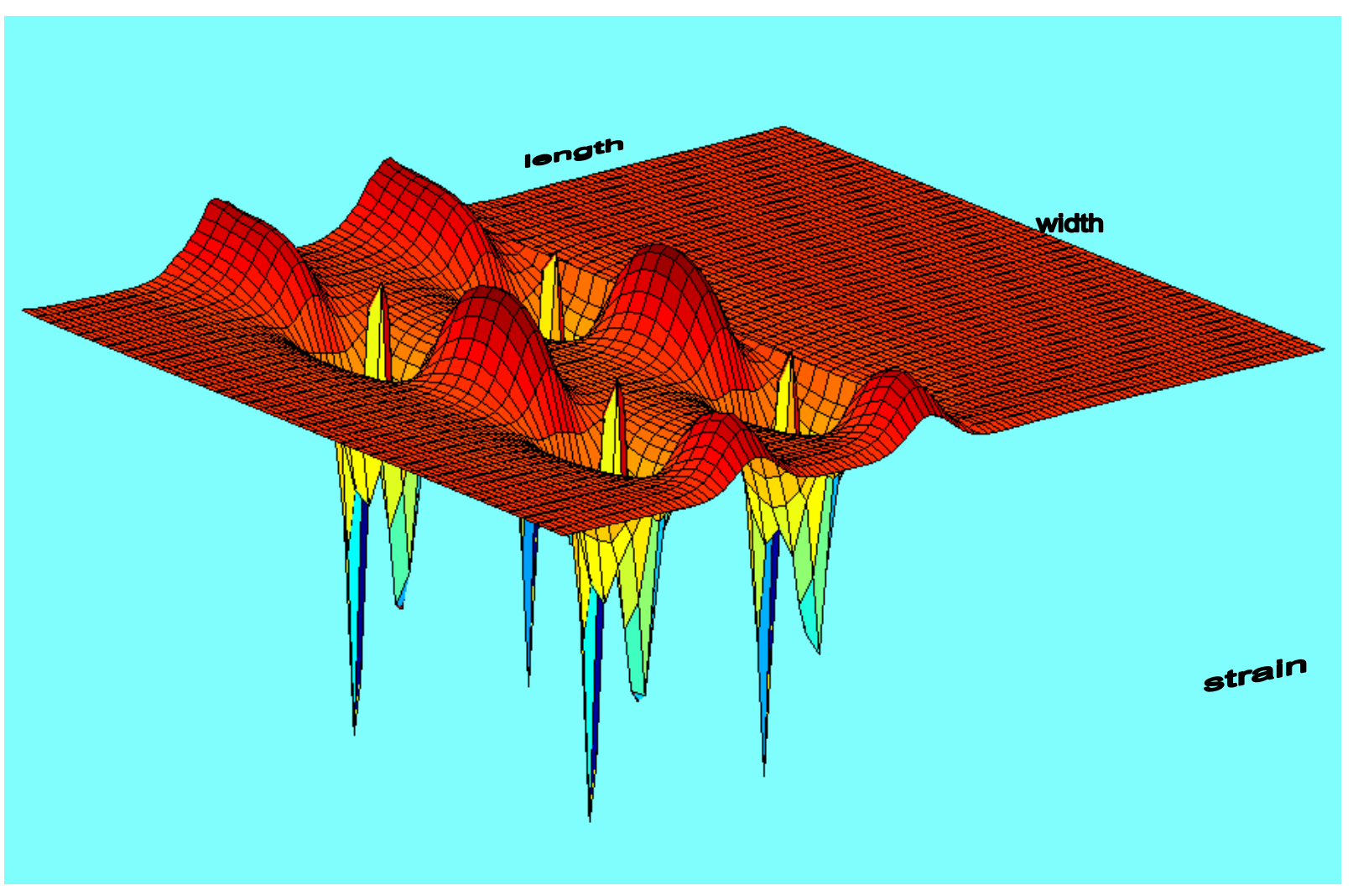

c. vertical strain

Appendix C - 5 3D stress strain states with multiple wheel load 
Lateral Stress (pa)

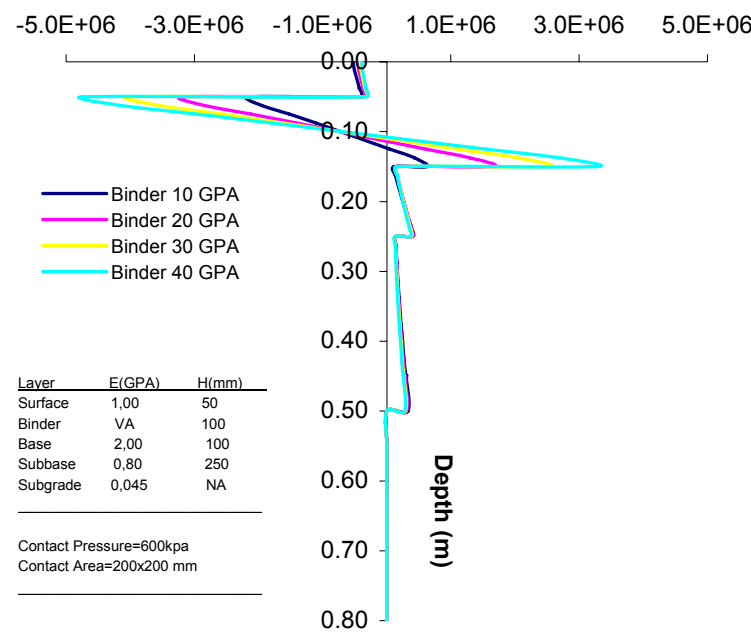

Vertical Stress (pa)

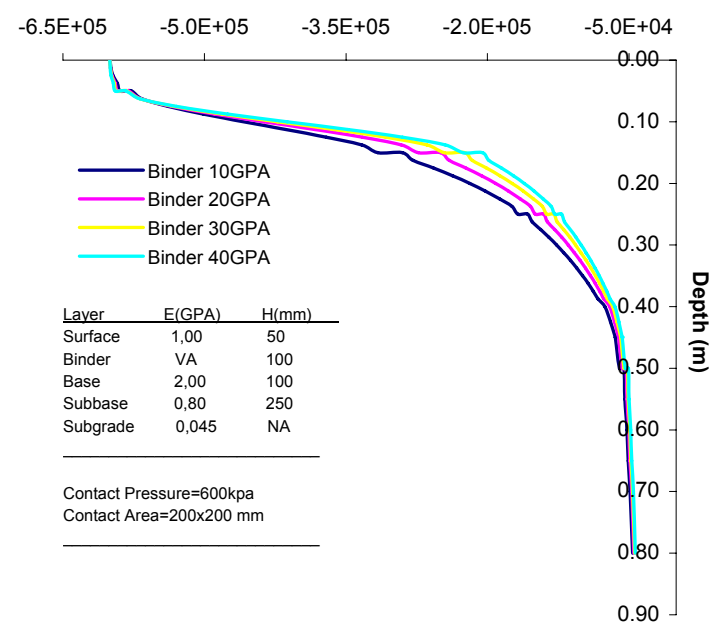

Shear Stress (pa)

$\begin{array}{llllll}-5.0 \mathrm{E}+05 & -4.0 \mathrm{E}+05 & -3.0 \mathrm{E}+05 & -2.0 \mathrm{E}+05 & -1.0 \mathrm{E}+05 & 0.0 \mathrm{E}+00\end{array}$

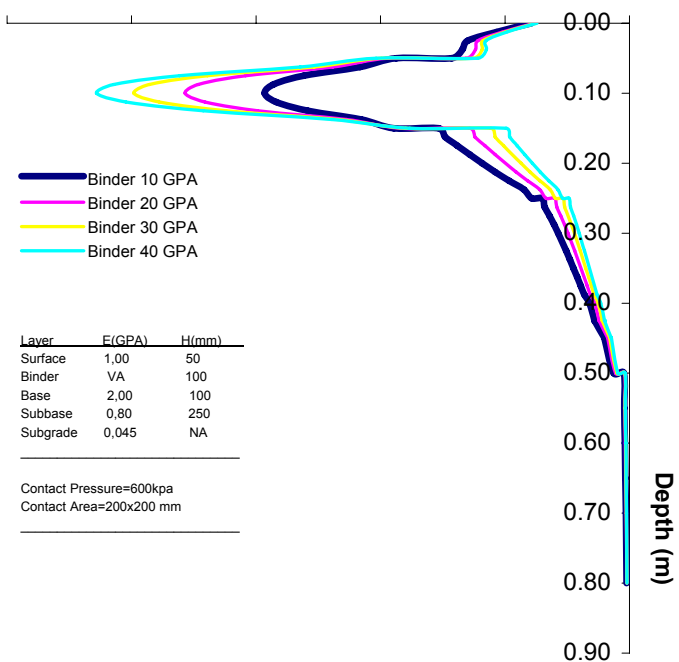

Lateral Strain $(\mathrm{mm} / \mathrm{mm})$

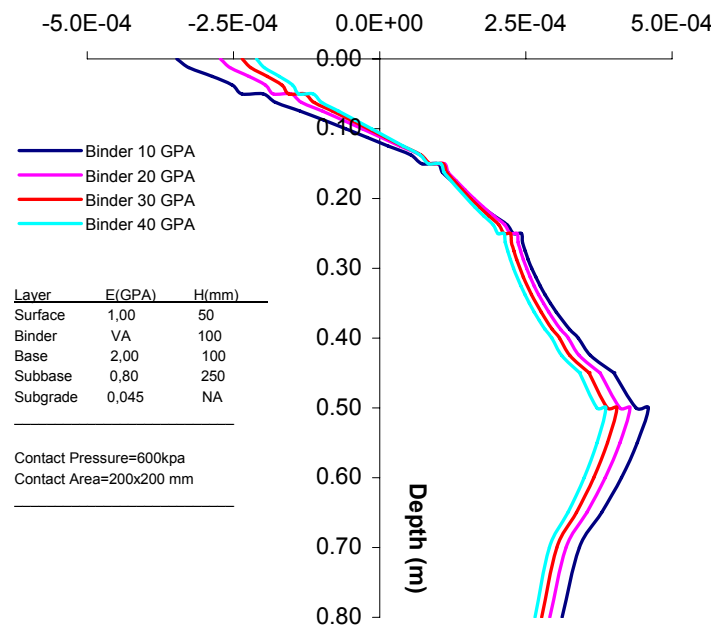

Vertical strain $(\mathrm{mm} / \mathrm{mm})$

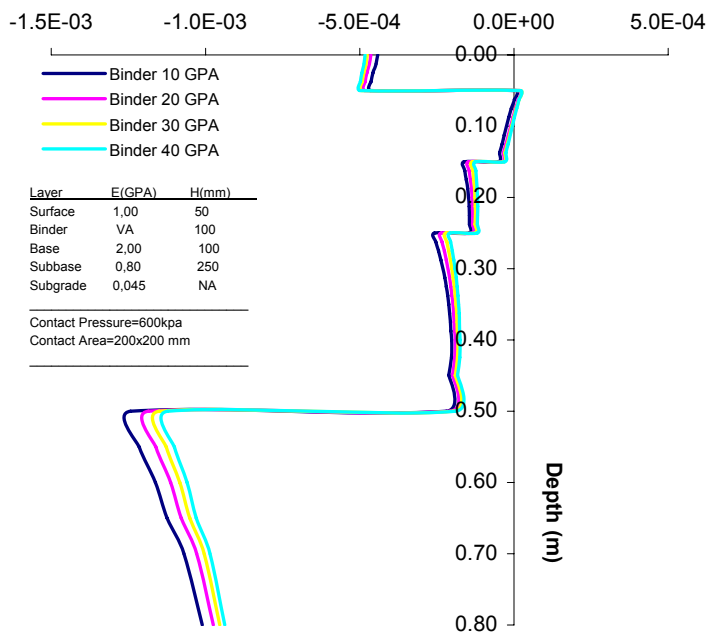

Shear Strain ()

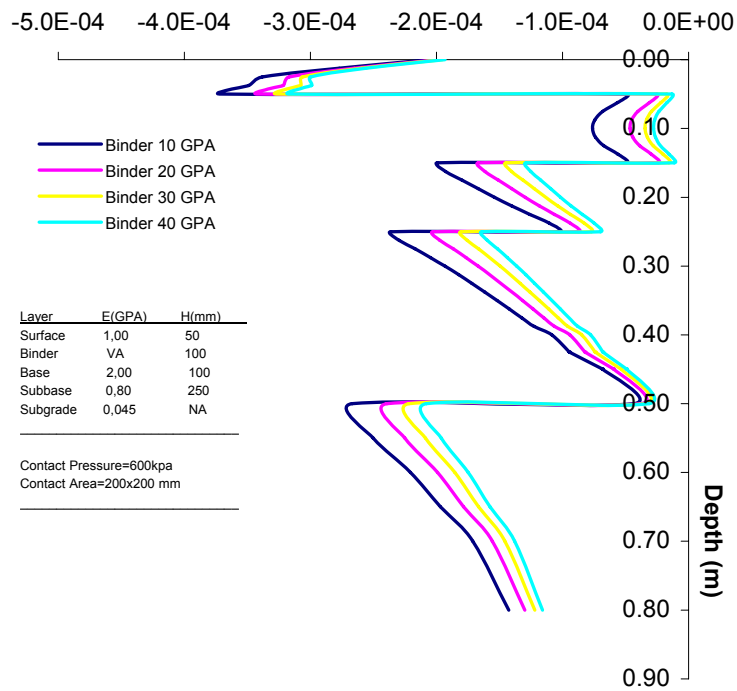

Appendix C - 6 Stress strain states with different layer moduli 

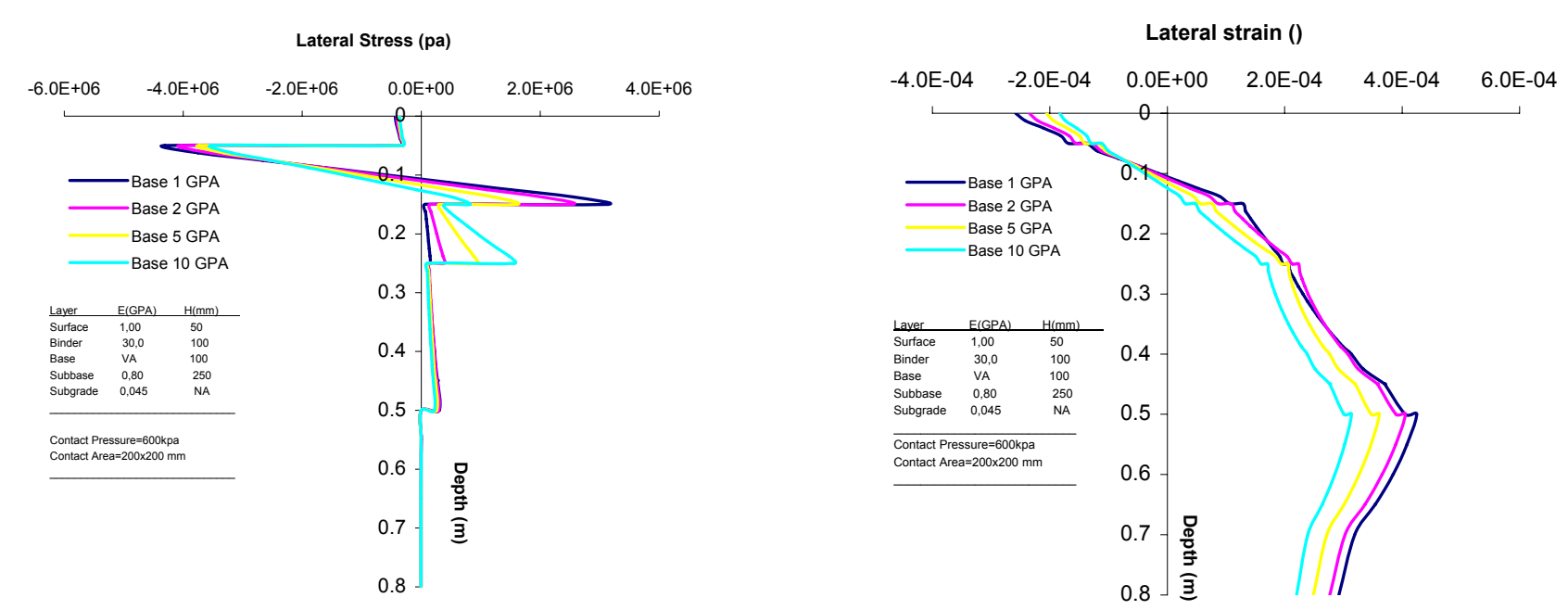

Normal Vertical Stress (pa)

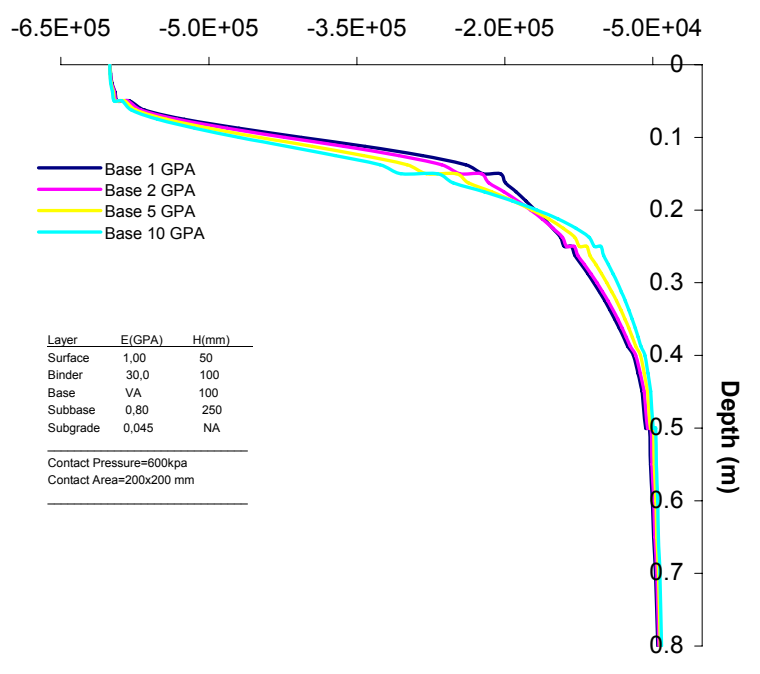

Normal Vertical Strain ()

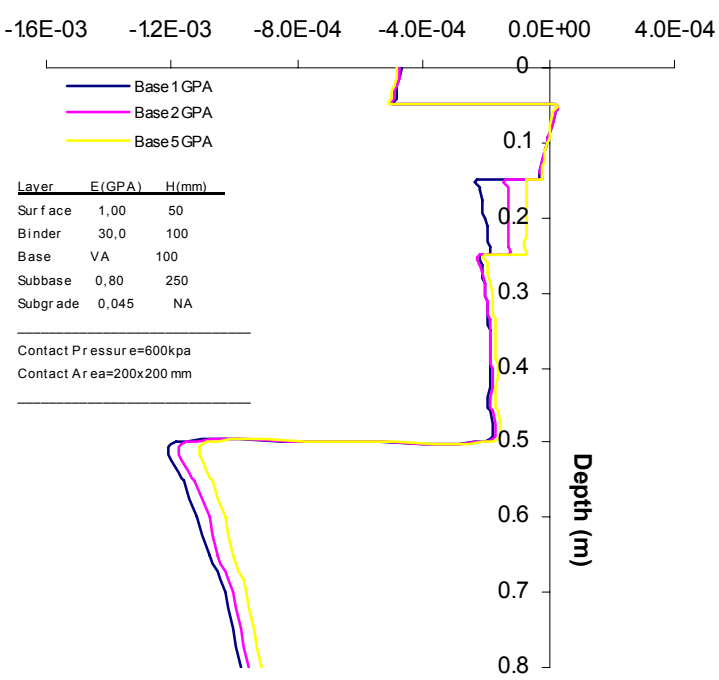

Shear Stress (pa)

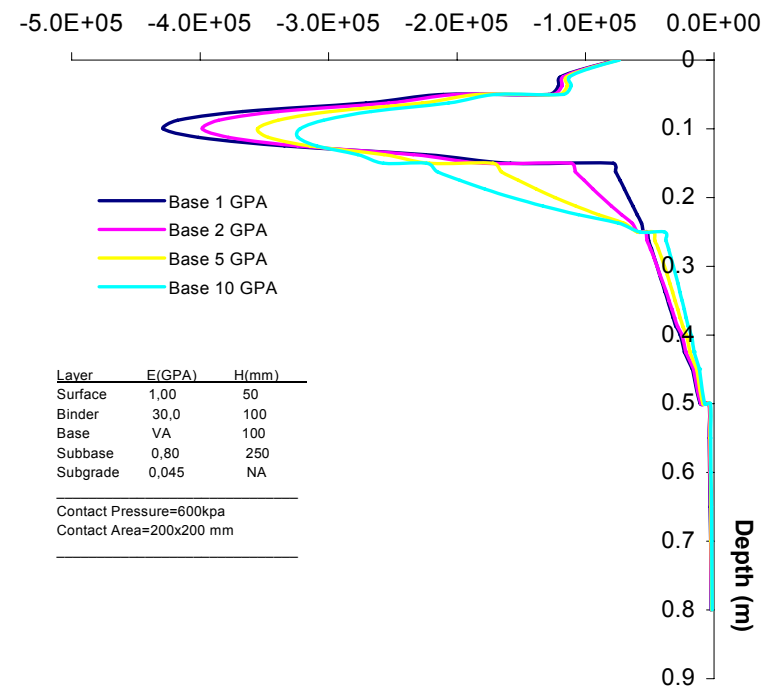

Shear Strain ()

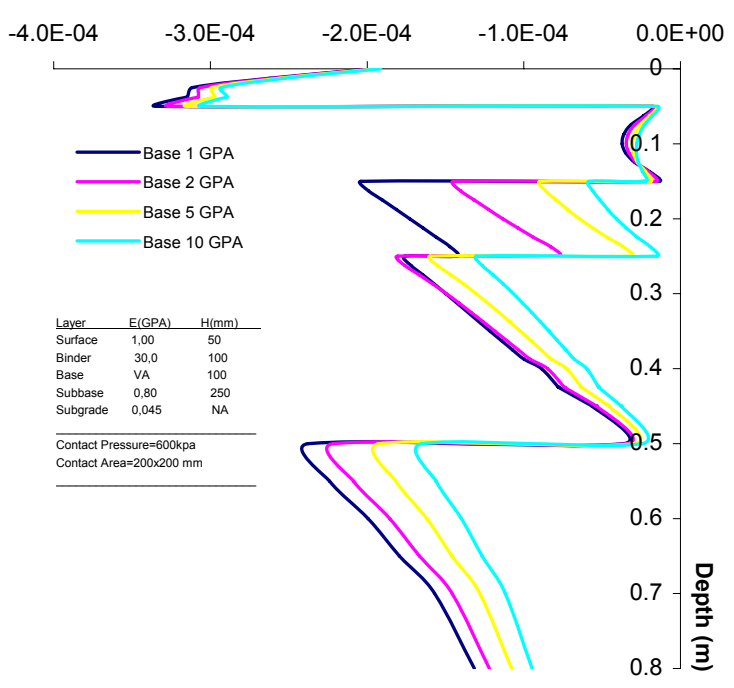

Appendix C - 7 Stress strain states with different layer moduli (continued) 


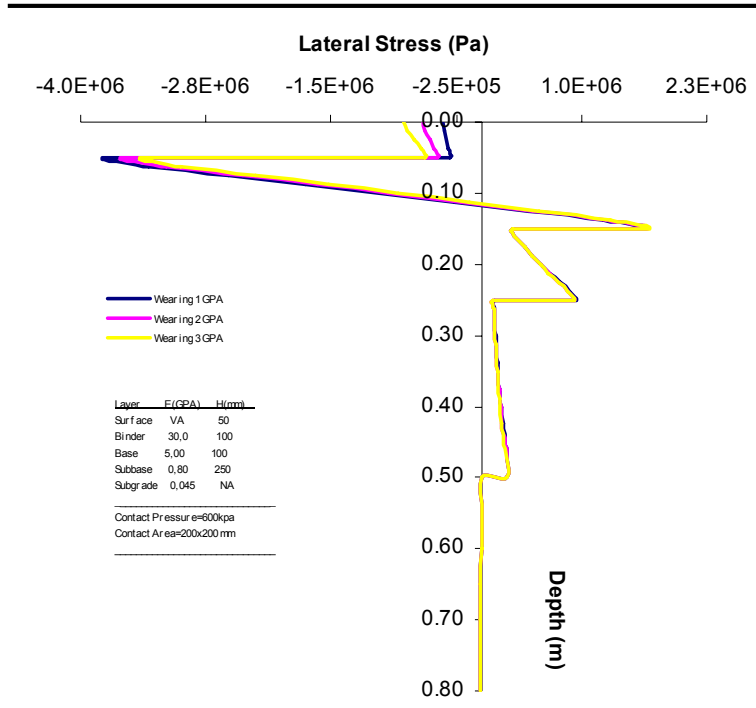

Lateral Strain ()
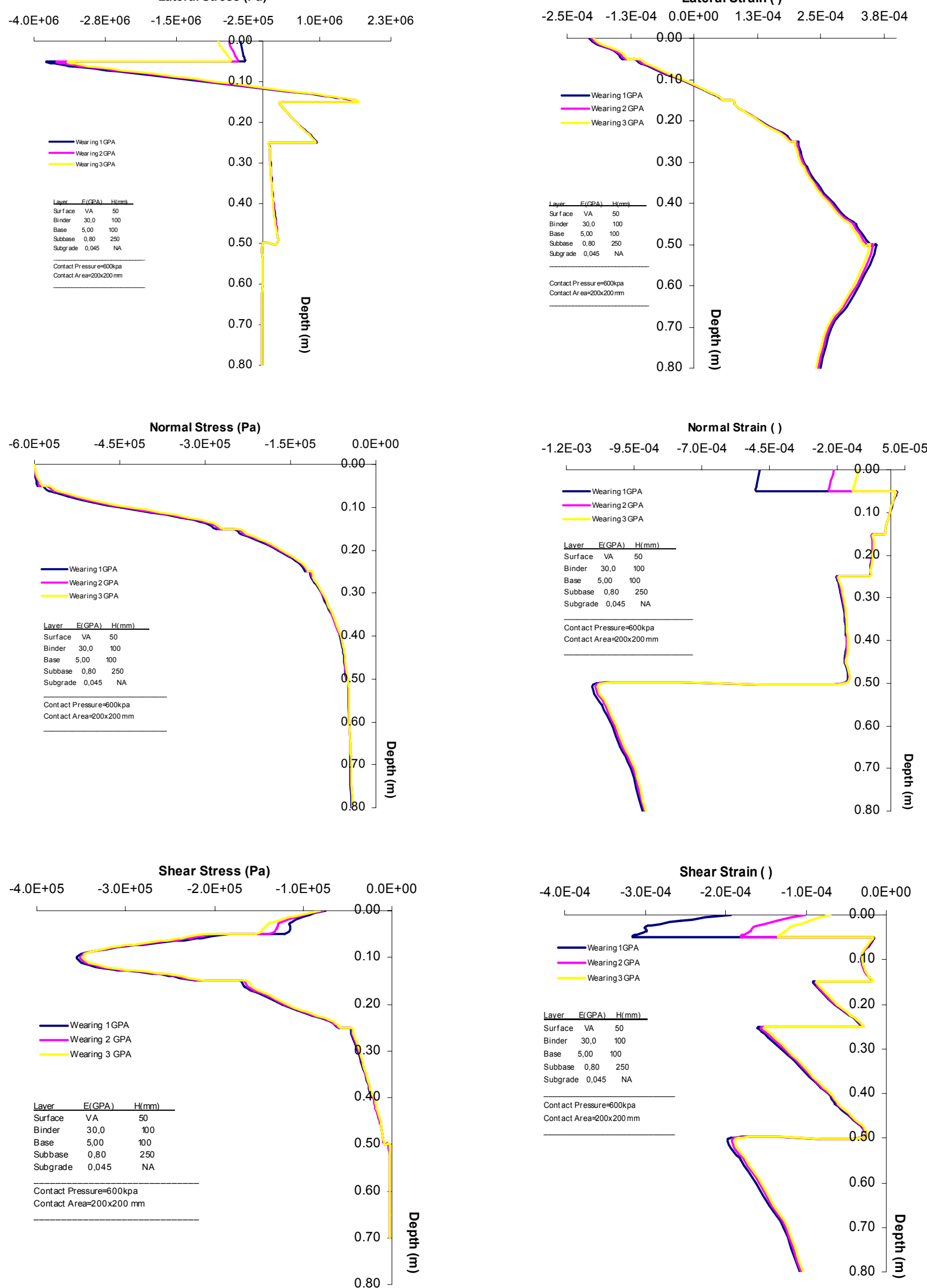

Appendix C - 8 Stress strain states with different layer moduli (continued) 

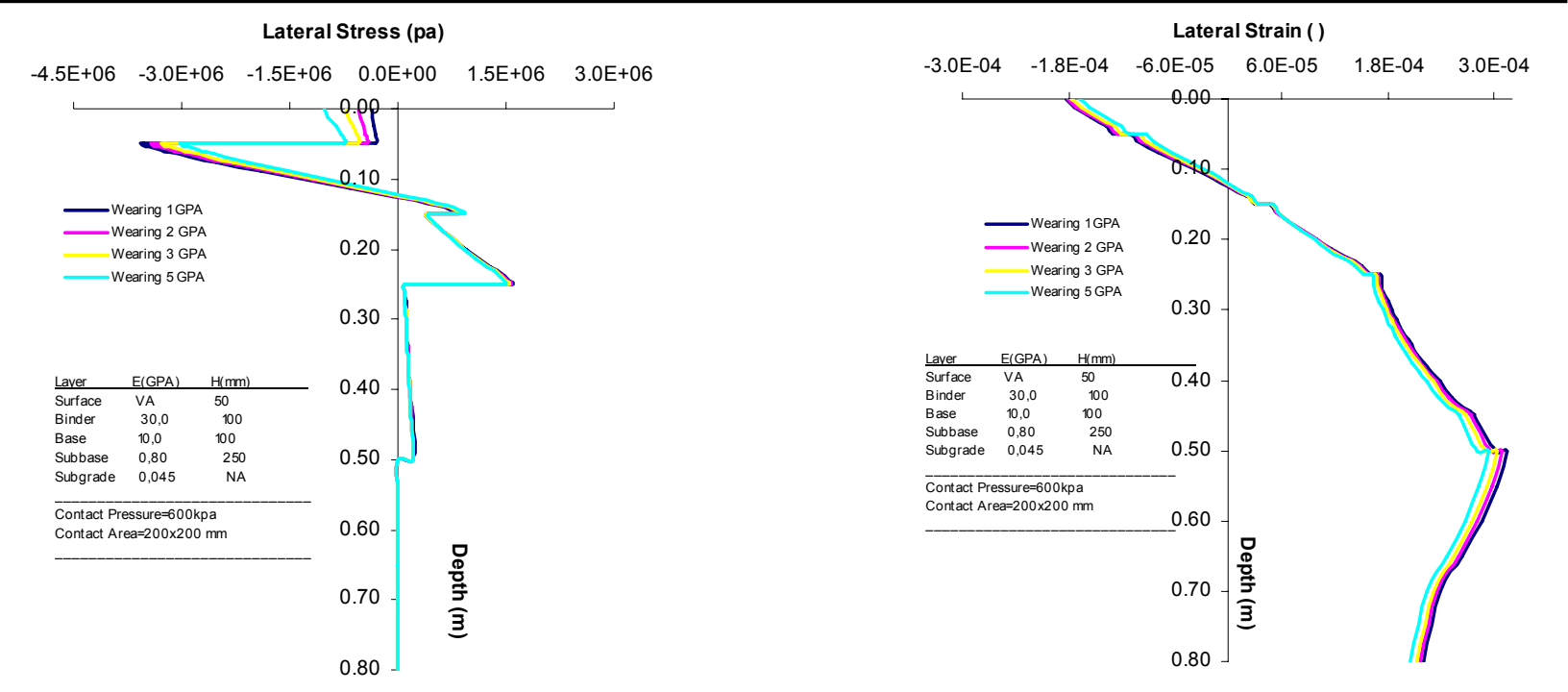

Normal Stress (pa)

Normal Strain ()

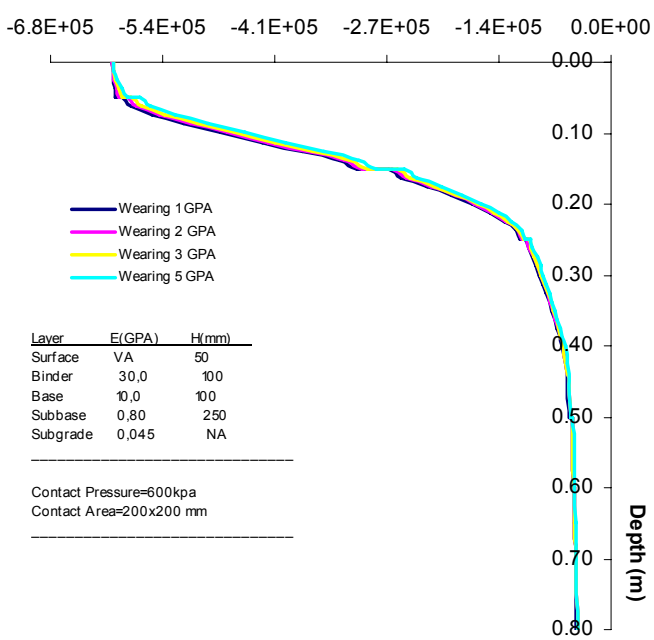

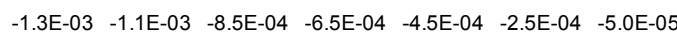

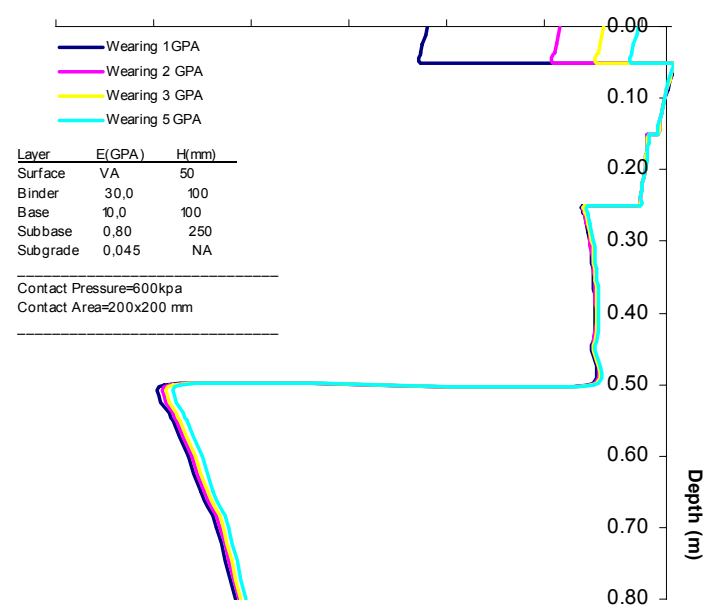

Shear Stress (pa)
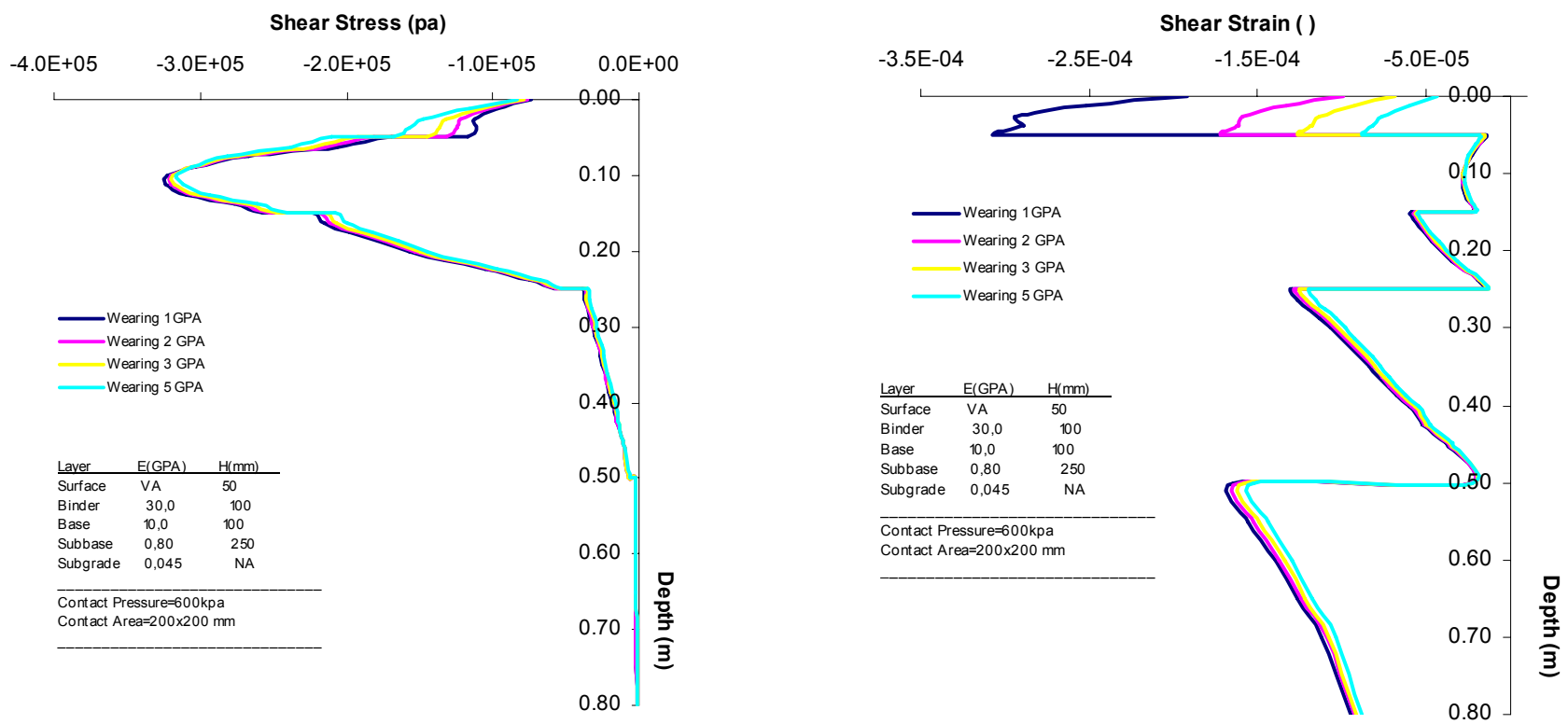

Appendix C - 9 Stress strain states with different layer moduli (continued) 


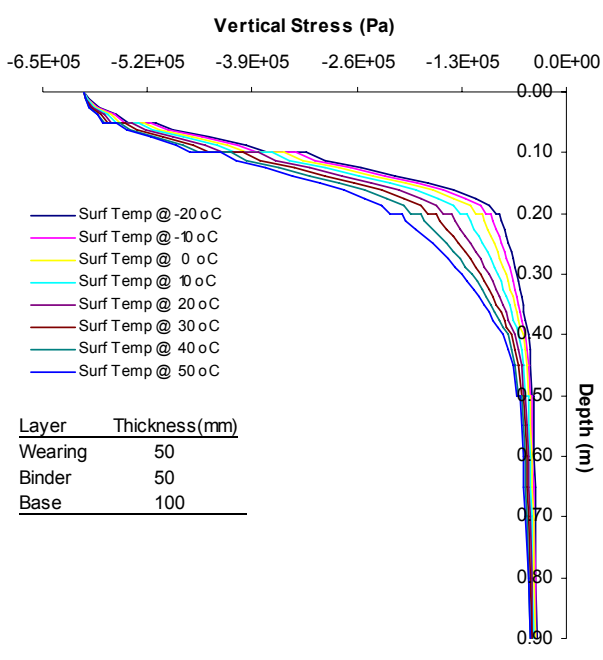

T5-5-10-Mt10

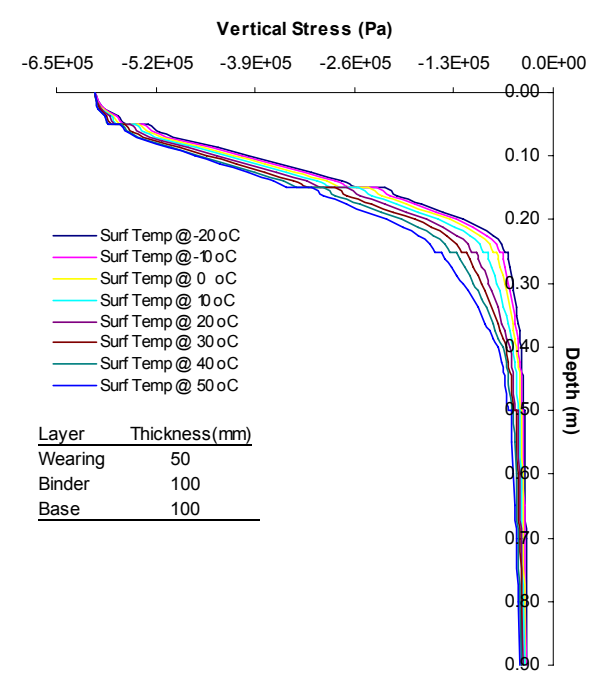

T5-10-10- Mt10

Vertical Stress $(\mathrm{Pa})$

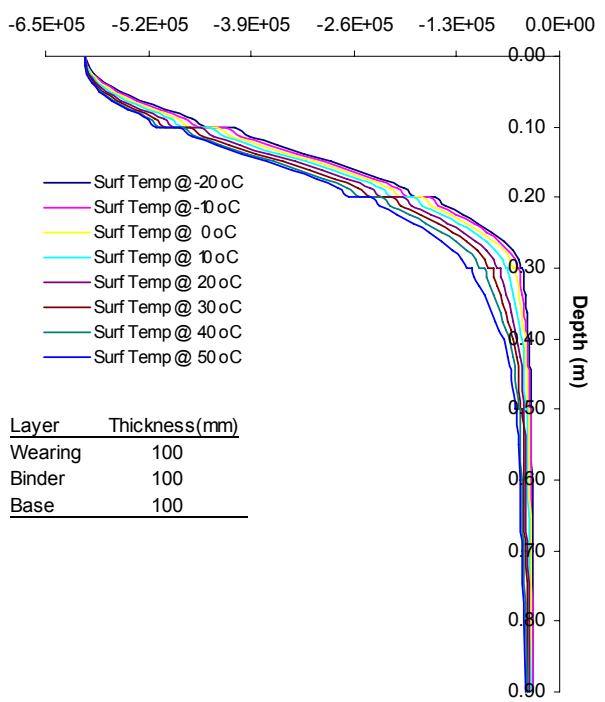

T10-10-10- Mt10

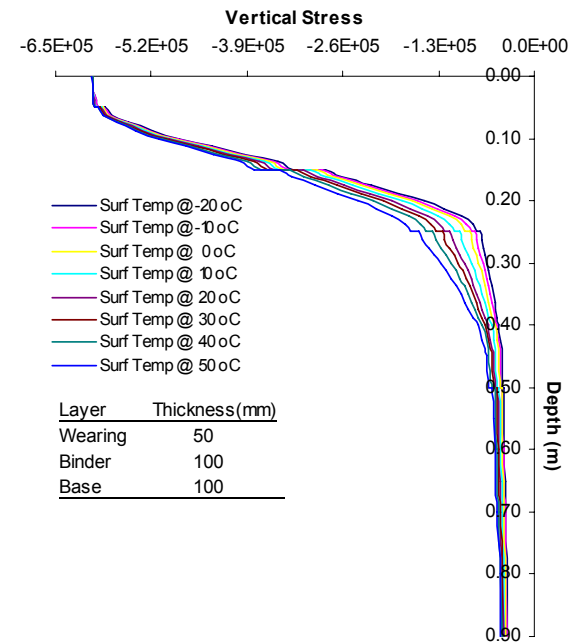

T5-10-10- Mtl1

Vertical Stress $(\mathrm{Pa})$

$-6.4 \mathrm{E}+05 \quad-4.8 \mathrm{E}+05 \quad-3.2 \mathrm{E}+05 \quad-1.6 \mathrm{E}+05 \quad 0.0 \mathrm{E}+00$

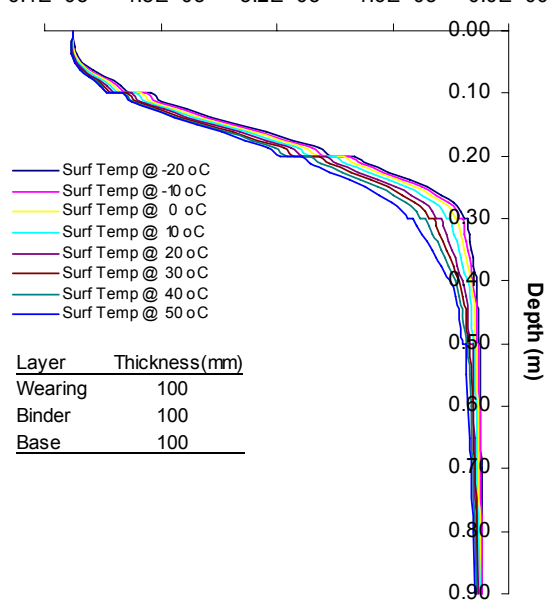

T10-10-10- MtlE2

Vertical Stress $(\mathrm{Pa})$

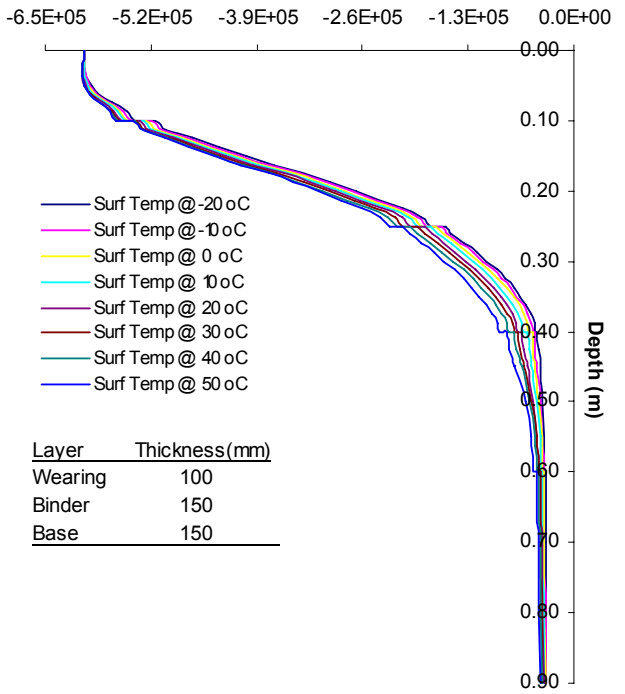

T10-15-15- MtlE2

Appendix C - 10 FE result used for model input 
Appendix D: Temperature Variables and Rutting Model Output
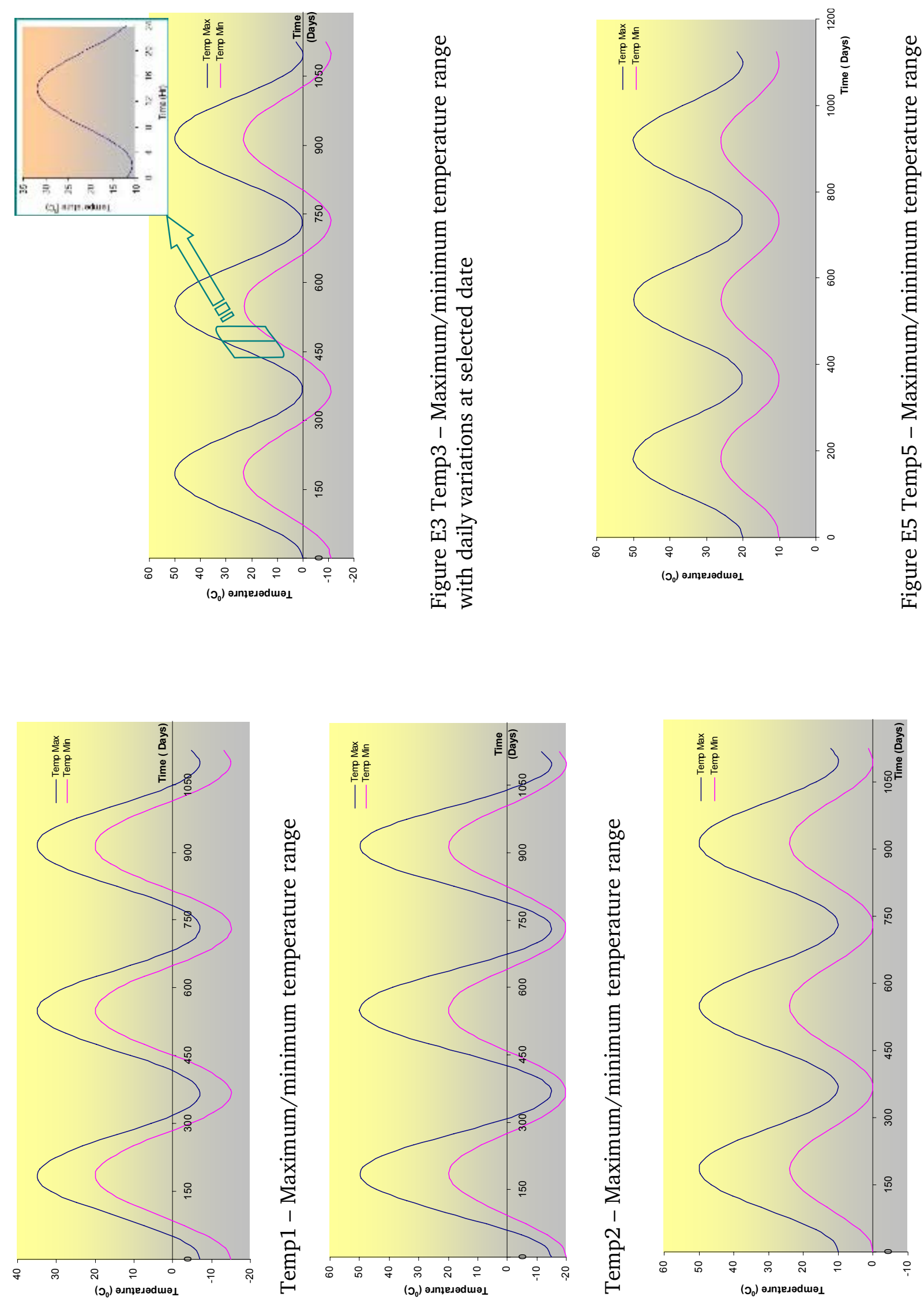

告 

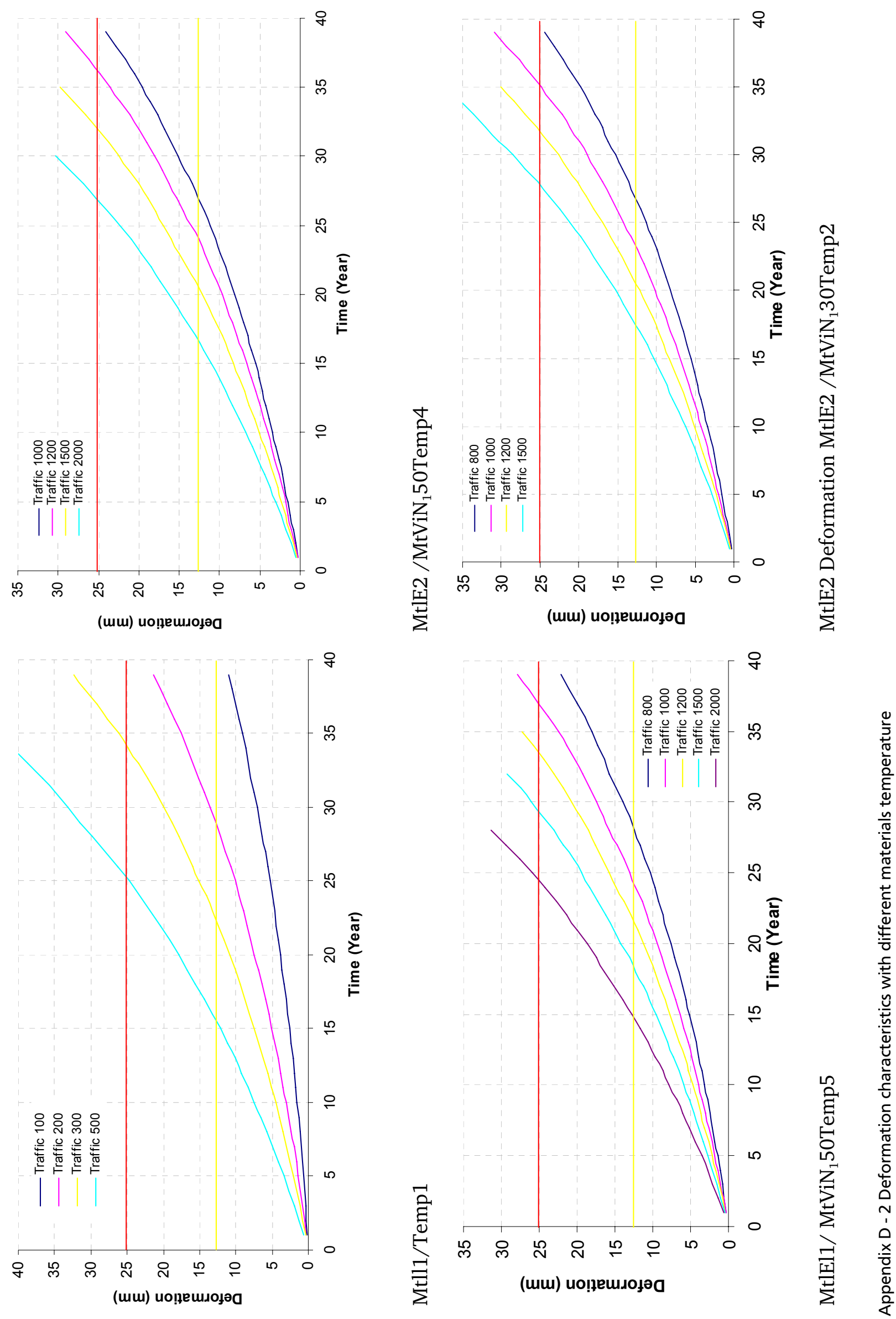

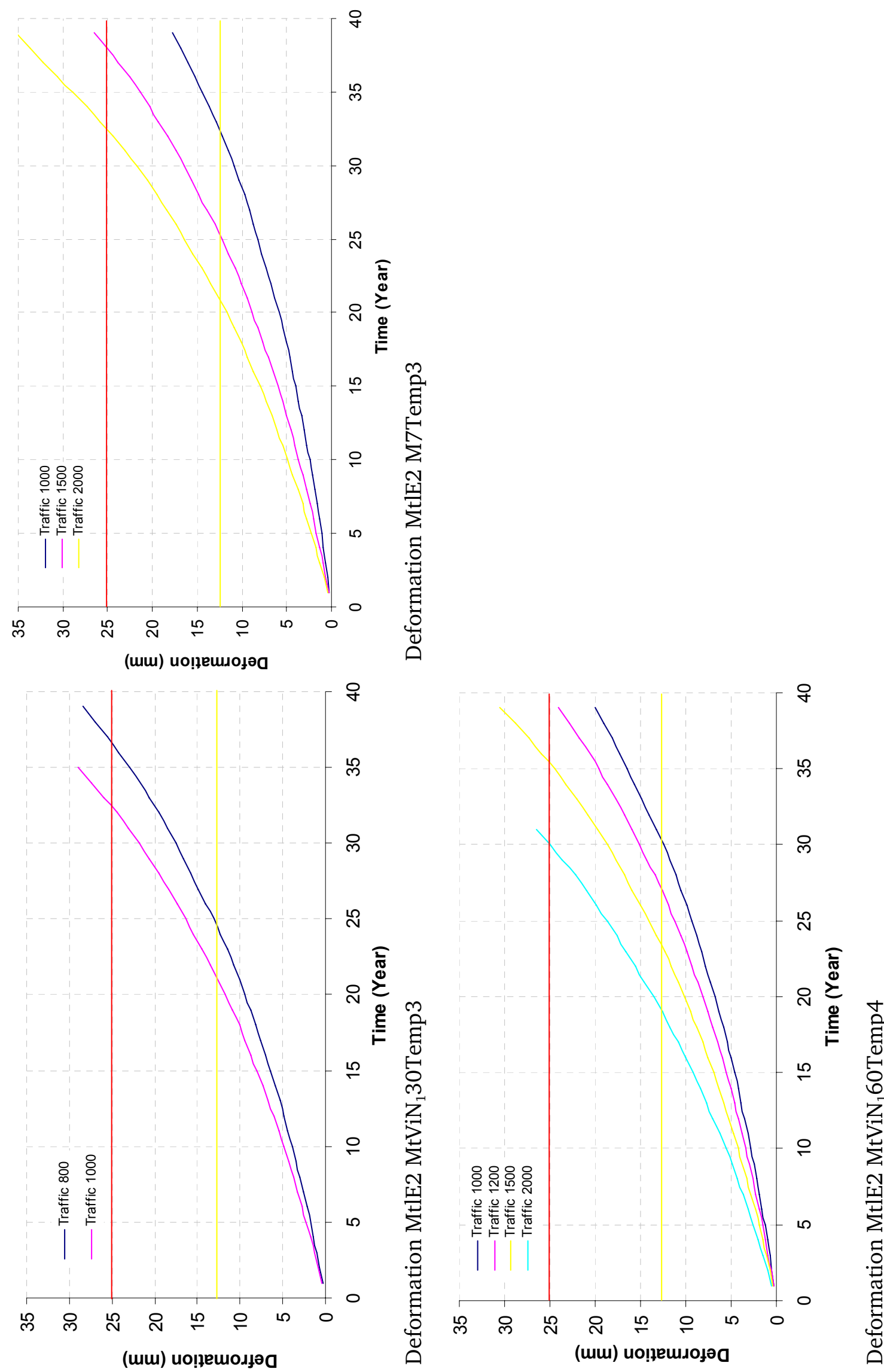

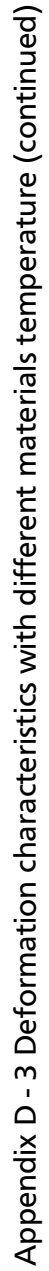


Appendix E: Existing Rut Prediction Models

\begin{tabular}{|c|c|c|c|c|c|c|}
\hline Author & $\begin{array}{c}\text { Pavement } \\
\text { Analysis }\end{array}$ & $\begin{array}{c}\text { Permanent } \\
\text { Deformation } \\
\text { Equation }\end{array}$ & & Variables & $\begin{array}{l}\text { Laboratory } \\
\text { Test }\end{array}$ & Observations \\
\hline $\begin{array}{l}\text { Kirwan } \\
\text { Snaith } \\
\text { Glynn } \\
(1977)\end{array}$ & $\begin{array}{l}\text { DEFPAV } \\
\text { Nonlinear } \\
\text { finite } \\
\text { element } \\
\text { Layer strain } \\
\text { method }\end{array}$ & $e_{n}=A \Delta^{b}$ & $\begin{array}{l}e_{\mathrm{B}} \\
\mathrm{A} \\
\mathrm{b} \\
\mathrm{A}\end{array}$ & $\begin{array}{l}=\quad \begin{array}{l}\text { induced axial } \\
\text { permanent strain } \\
\text { after an elapsed } \\
\text { time } \mathrm{N}\end{array} \\
\text { function of elapsed } \\
\text { time and material } \\
=\quad \begin{array}{l}\text { constant for the } \\
\text { material }\end{array} \\
=\quad \begin{array}{l}\text { applied axial } \\
\text { compressive stress }\end{array}\end{array}$ & $\begin{array}{l}\text { Uniaxial } \\
\text { compression } \\
\text { dynamic } \\
\text { loading creep } \\
\text { test }\end{array}$ & $\begin{array}{l}\text { Calculated } \\
\text { values of rut } \\
\text { depth higher } \\
\text { than obtained } \\
\text { in Nottingham } \\
\text { Test Track }\end{array}$ \\
\hline $\begin{array}{l}\text { Monismith } \\
\text { Inkabi } \\
\text { Freeme } \\
\text { McLean } \\
\text { (1977) }\end{array}$ & \begin{tabular}{|l} 
ELSYM \\
Layered \\
elastic theory \\
Layer strain \\
method
\end{tabular} & $\begin{aligned} e_{z} & \left.=\left[\delta(T) N^{\alpha} \bar{\sigma}^{n-1}\right]\right] \\
& \cdot\left[\sigma_{x}-\frac{1}{2}\left(\sigma_{x}+\sigma\right)\right]\end{aligned}$ & $\begin{array}{l}e_{i}^{p} \\
\delta(T) \\
\alpha \\
N \\
\bar{\sigma} \\
\end{array}$ & $\begin{array}{l}=\begin{array}{l}\text { vertical permanent } \\
\text { deformation } \\
\text { function of } \\
\text { temperature } \\
\text { coefficient } \\
\text { determined } \\
\text { experimentally } \\
\text { number of stress } \\
\text { repetitions }\end{array} \\
=\begin{array}{l}\text { equivalent stress } \\
\text { defined as a } \\
\text { function of } \sigma_{1}, \sigma_{2}\end{array} \\
=\begin{array}{l}\text { and } \sigma_{3} \\
\text { loading time }\end{array} \\
=\quad \begin{array}{l}\text { long } \\
=\end{array}\end{array}$ & $\begin{array}{l}\text { Repeated load } \\
\text { triaxial } \\
\text { compression } \\
\text { test }\end{array}$ & $\begin{array}{l}\text { Consideration } \\
\text { of traffic axde } \\
\text { load and } \\
\text { lateral } \\
\text { distribution }\end{array}$ \\
\hline \begin{tabular}{|l|} 
Brown \\
Bell \\
$(1979)$
\end{tabular} & $\begin{array}{l}\text { Computer } \\
\text { program } \\
\text { DEFPAV } \\
\text { Nonlinear } \\
\text { finite element } \\
\text { Layer strain } \\
\text { method } \\
\end{array}$ & $e_{p}=\left(\frac{q}{a}\right)^{b}(M)$ & $\begin{array}{l}e \\
q \\
a, b \\
N\end{array}$ & 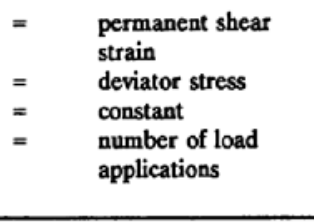 & $\begin{array}{l}\text { Axial repeated } \\
\text { load tests }\end{array}$ & $\begin{array}{l}\text { Relatively } \\
\text { good } \\
\text { agreement in } \\
\text { test track }\end{array}$ \\
\hline $\begin{array}{l}\text { Meyer } \\
\text { Haas } \\
(1977)\end{array}$ & $\begin{array}{l}\text { Computer } \\
\text { program } \\
\text { FEPAVE II } \\
\text { Finite element } \\
\text { Layer strain } \\
\text { method }\end{array}$ & $e_{p}=F\left(\sigma_{1}, \sigma_{y}, T, A V_{3}, N\right) \pm E$ & $\begin{array}{l}\text { ep } \\
\sigma_{1} \\
\sigma_{3} \\
\text { T } \\
\text { AV } \\
\text { N } \\
\text { E }\end{array}$ & $\begin{array}{ll}= & \text { axial permanent } \\
& \text { deformation } \\
= & \text { vertical stress } \\
= & \text { lateral stress } \\
= & \text { temperature } \\
= & \text { air voids } \\
= & \text { number of load } \\
& \text { applications } \\
= & \text { error of estimate }\end{array}$ & $\begin{array}{l}\text { Repeated load } \\
\text { triaxial test }\end{array}$ & $\begin{array}{l}\text { Measured } \\
\text { values for rut } \\
\text { depth on the } \\
\text { Brampton } \\
\text { Test Road } \\
\text { sections. } \\
\text { Good } \\
\text { agreement } \\
\text { between } \\
\text { measured and } \\
\text { predicted. }\end{array}$ \\
\hline $\begin{array}{l}\text { van de Loo } \\
(1976)\end{array}$ & $\begin{array}{l}\text { BISAR } \\
\text { Elastic layer } \\
\text { theory }\end{array}$ & $e_{p}=c \sigma N^{a}$ & $\begin{array}{l}\text { e } \\
\text { c } \\
\text { a } \\
\text { N } \\
\text { a }\end{array}$ & $\begin{array}{l}=\quad \begin{array}{l}\text { axial permanent } \\
\text { deformation }\end{array} \\
=\quad \begin{array}{l}\text { constant } \\
\text { axial stress level (15 }\end{array} \\
=\quad \begin{array}{l}\text { PSI) } \\
\text { number of load }\end{array} \\
=\begin{array}{l}\text { applications } \\
\text { constant }\end{array}\end{array}$ & $\begin{array}{l}\text { Axial creep } \\
\text { test }\end{array}$ & $\begin{array}{l}\text { Basis of } \\
\text { SHELL } \\
\text { method. } \\
\text { Generally } \\
\text { overestimates } \\
\text { rut depth. }\end{array}$ \\
\hline $\begin{array}{l}\text { Kenis } \\
(1977)\end{array}$ & $\begin{array}{l}\text { VESYS } \\
\text { Probabilistic } \\
\text { linear } \\
\text { viscoelastic } \\
\text { solution }\end{array}$ & $\epsilon_{,}(N)=e \mu N^{-\alpha}$ & $\begin{array}{l}e_{p}(\mathrm{~N}) \\
\alpha \\
s \\
e \\
\mu \\
\mathrm{I} \\
\end{array}$ & 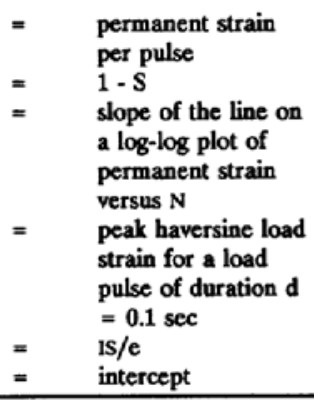 & $\begin{array}{l}\text { Uniaxial } \\
\text { repeated load } \\
\text { tests }\end{array}$ & $\begin{array}{l}\text { Basis of the } \\
\text { VESYS } \\
\text { approach }\end{array}$ \\
\hline
\end{tabular}

Appendix E - 1 Permanent deformation models developed by several Authors [31] 


\begin{tabular}{|c|c|c|c|c|c|c|c|}
\hline $\begin{array}{l}\text { Francken } \\
\text { (1977) }\end{array}$ & & $\begin{array}{l}e_{p}(t)=A t^{B}+C(\exp D t-1) \\
(\text { high stresses) } \\
e_{p}(t)=A t^{B} \\
(\text { low stresses })\end{array}$ & $\begin{array}{l}\mathbf{e}_{\mathrm{p}}(\mathrm{t}) \\
\mathrm{A}, \mathrm{B}, \mathrm{C}, \mathrm{D} \\
\mathrm{A} \\
\mathrm{B} \\
\sigma_{\mathrm{VM}} \\
\sigma_{\mathrm{VL}}\end{array}$ & $\begin{array}{l}= \\
= \\
= \\
= \\
= \\
=\end{array}$ & $\begin{array}{l}\text { permanent strain } \\
\text { parameters } \\
115\left(\sigma_{1}-\sigma_{3}\right) /\left|\mathrm{E}^{*}\right| \\
.182+.294\left(\sigma_{\mathrm{VM}}-\right. \\
\left.\sigma_{\mathrm{VL}}\right) \\
\text { maximum stress } \\
\text { plastic failure } \\
\text { threshold }\end{array}$ & $\begin{array}{l}\text { Triaxial } \\
\text { dynamic tests }\end{array}$ & $\begin{array}{l}\text { Method used } \\
\text { to determine } \\
\text { rutting } \\
\text { propensity in } \\
\text { mixes }\end{array}$ \\
\hline $\begin{array}{l}\text { Verstraeten } \\
\text { Romain } \\
\text { Veverka } \\
(1982)\end{array}$ & $\begin{array}{l}\text { Computer } \\
\text { program } \\
\text { ORN093 } \\
\text { Elastic layer } \\
\text { theory } \\
\text { Layer strain } \\
\text { theory }\end{array}$ & $e_{p}(t)=A\left(\frac{t}{10^{3}}\right)^{B}=\frac{C\left(\sigma_{1}-\sigma_{3}\right)}{\left|E^{*}\right| *\left(\frac{t}{10^{3}}\right)^{*}}$ & $\begin{array}{l}e_{p}(t) \\
A \\
\\
\\
B \\
\text { B } \\
\left|E^{*}\right| \\
\sigma_{1} \\
\sigma_{3}\end{array}$ & $\begin{array}{l}= \\
= \\
= \\
= \\
= \\
= \\
=\end{array}$ & $\begin{array}{l}\text { permanent strain at } \\
\text { time } t \text { (in sec) } \\
\text { is a coefficient } \\
\text { depending on the } \\
\text { mix composition } \\
\text { and on the } \\
\text { experimental } \\
\text { conditions (stresses, } \\
\text { frequency, } \\
\text { temperature); it } \\
\text { characterizes the } \\
\text { susceptibility of the } \\
\text { mix to rutting } \\
\text { is a coefficient } \\
\text { varying between } .14 \\
\text { and } .37 \\
\left.\text { f } \mathrm{v}_{\mathrm{b}} /\left(\mathrm{v}_{\mathrm{b}}+\mathrm{v}_{\mathrm{v}}\right)\right] \\
\text { modulus of the mix } \\
\text { amplitude of } \\
\text { vertical stress } \\
\text { lateral stress }\end{array}$ & $\begin{array}{l}\text { Triaxial } \\
\text { dynamic tests }\end{array}$ & $\begin{array}{l}\text { Acceptable } \\
\text { correlation } \\
\text { with rut depth } \\
\text { measure in } 16 \\
\text { in-service } \\
\text { roads }\end{array}$ \\
\hline $\begin{array}{l}\text { Huschek } \\
\text { (1977) }\end{array}$ & $\begin{array}{l}\text { BISAR } \\
\text { Elastic layer } \\
\text { theory } \\
\text { Layer strain } \\
\text { theory }\end{array}$ & $\begin{array}{c}e_{t r r}=c \cdot \sigma t^{A} \\
e_{l r}\left(T, \Delta t_{1}, t\right)=\frac{\sigma \Delta t_{1}}{[\eta(T, t)]} \\
\eta(T, t)=\frac{t^{1-A}}{(c \cdot A)}\end{array}$ & $\begin{array}{l}\mathrm{e}_{\mathrm{ir}} \\
\mathrm{c} \\
\mathrm{A} \\
\mathbf{\sigma} \\
\eta \\
\mathrm{T} \\
\Delta_{\mathrm{t}_{1}}\end{array}$ & $\begin{array}{l}= \\
= \\
= \\
= \\
= \\
= \\
=\end{array}$ & $\begin{array}{l}\text { permanent } \\
\text { deformation } \\
\text { constant } \\
\text { consolidation } \\
\text { characteristic } \\
\text { stress level } \\
\text { viscosity } \\
\text { temperature } \\
\text { time of loading }\end{array}$ & $\begin{array}{l}\text { Uniaxial creep } \\
\text { tests } \\
\text { Cyclic load } \\
\text { creep test }\end{array}$ & $\begin{array}{l}\text { Asphalt mix is } \\
\text { represented by } \\
\text { a Maxwell } \\
\text { element: } \\
\text { spring and } \\
\text { dashpot in } \\
\text { series }\end{array}$ \\
\hline $\begin{array}{l}\text { Thrower } \\
\text { (1977) }\end{array}$ & $\begin{array}{l}\text { Viscoelastic } \\
\text { theory } \\
\text { Separative } \\
\text { method }\end{array}$ & $\begin{array}{c}\dot{c}_{i j}=\frac{\sigma_{i j}}{2 \eta} i \neq j \\
\dot{e}_{i j}=\frac{\sigma_{m}}{3 x}+\frac{\left(9 \sigma_{y}-\sigma_{m}\right)}{18 \eta}\end{array}$ & $\begin{array}{l}\dot{\mathrm{e}}_{\mathrm{ij}} \\
\sigma_{\mathrm{ij}} \\
\sigma_{\mathrm{m}} \\
x \\
\eta\end{array}$ & $\begin{array}{l}= \\
= \\
= \\
= \\
=\end{array}$ & $\begin{array}{l}\text { rate of deformation } \\
\text { state of stress } \\
\text { isotropic mean } \\
\text { stress } \\
\text { coefficient of } \\
\text { "volume viscosity" } \\
\text { coefficient of shear } \\
\text { velocity }\end{array}$ & & \\
\hline $\begin{array}{l}\text { Battiato et } \\
\text { al. } \\
\text { (1977) }\end{array}$ & $\begin{array}{l}\text { Computer } \\
\text { program } \\
\text { MOREL } \\
\text { Viscoelastic } \\
\text { theory } \\
\text { Two layer } \\
\text { viscoelastic } \\
\text { incompressible } \\
\text { system }\end{array}$ & $\begin{array}{c}J(t)=J_{1} t^{*} \\
u_{i k}^{\text {perm }}=\frac{1}{\eta_{z}} \delta_{i k}(y, z)\end{array}$ & $\begin{array}{l}J(t) \\
t \\
J_{1} \\
\alpha \\
u_{i \mathbf{k}}^{\text {perm }} \\
\eta_{*} \\
g_{i k}(y, z) \\
\end{array}$ & $\begin{array}{l}= \\
= \\
= \\
=\end{array}$ & $\begin{array}{l}\text { creep compliance } \\
\text { function } \\
\text { time } \\
\text { shear creep } \\
\text { parameters } \\
\text { slope of line on a } \\
\text { log-log between } \mathrm{J}(\mathrm{t}) \\
\text { and time } \\
\text { permanent } \\
\text { deformation } \\
\text { shear viscosity of } \\
\text { the Maxwell } \\
\text { element in series } \\
\text { tensor function } \\
\end{array}$ & $\begin{array}{l}\text { Uniaxial creep } \\
\text { tests }\end{array}$ & $\begin{array}{l}\text { Asphalt mix is } \\
\text { represented by } \\
\text { a Maxwell } \\
\text { model }\end{array}$ \\
\hline
\end{tabular}

Appendix E - 2 Permanent deformation models developed by several Authors [31] (continued) 
Appendix F: Rut Modelling Program Flow Chart

Main Program Flow Chart

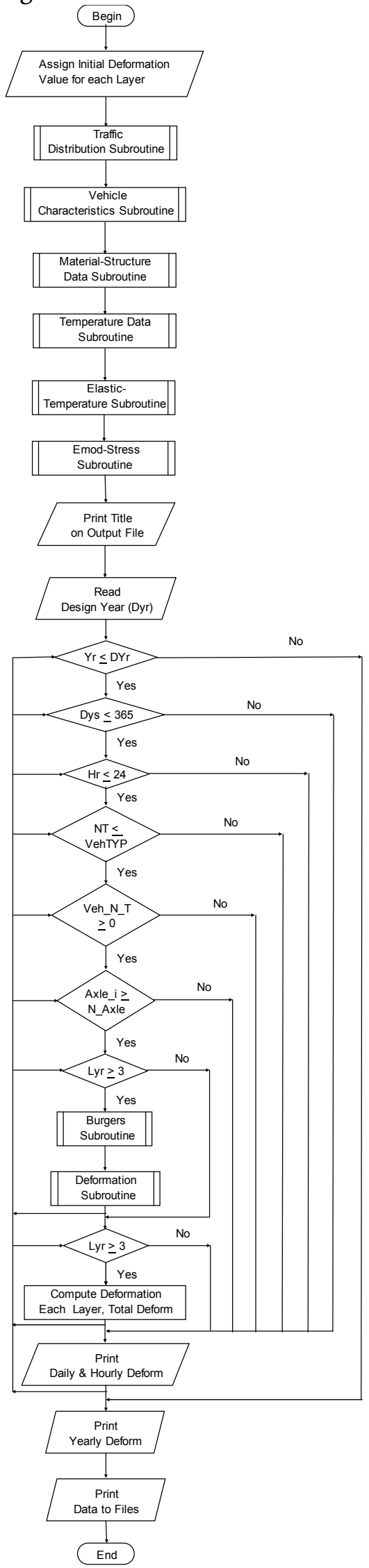

Traffic Distribution Flow Chart

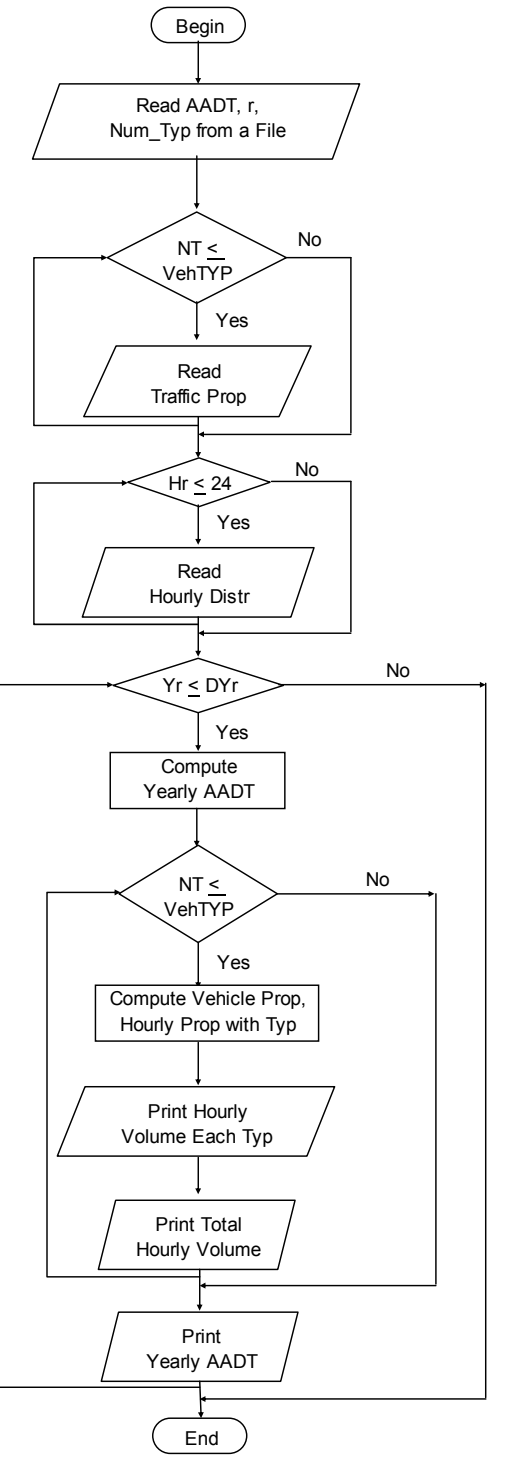

Appendix F - 1 Flow diagram 
Vehicle Characteristics Flow Chart

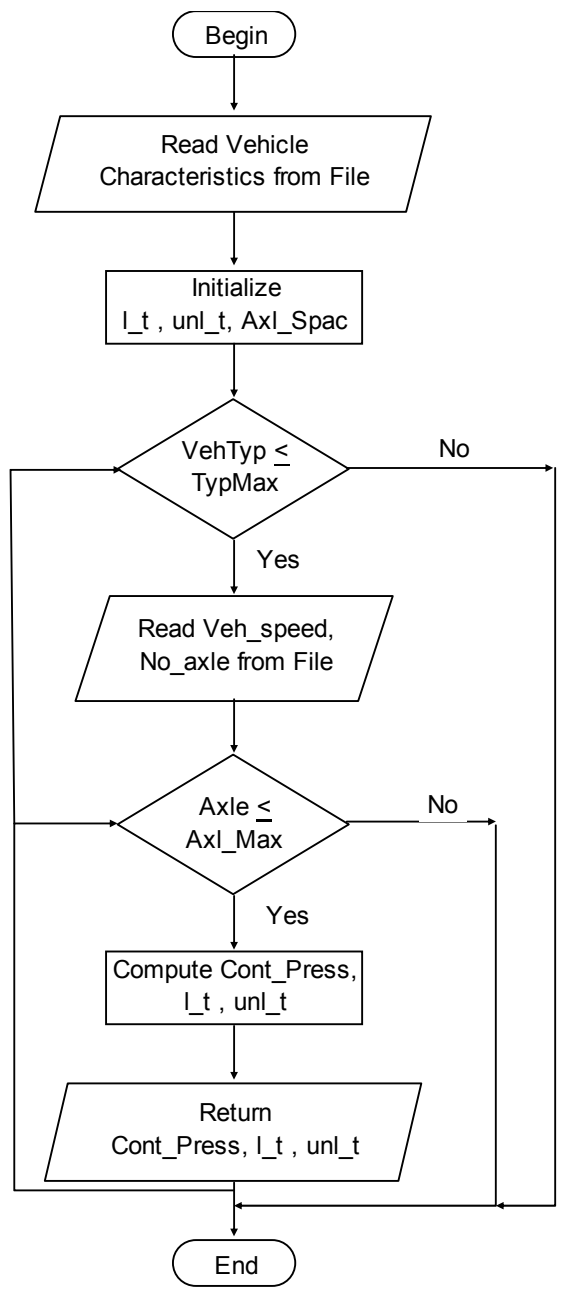

Temperature Flow Chart

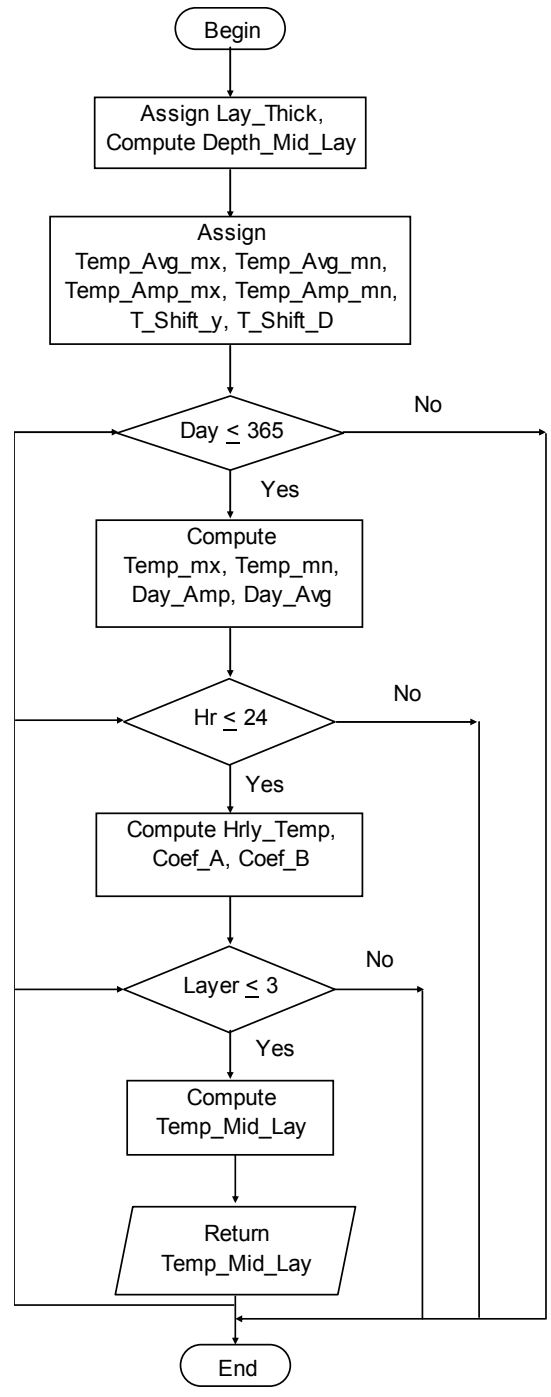

Mat_Struct Flow Chart

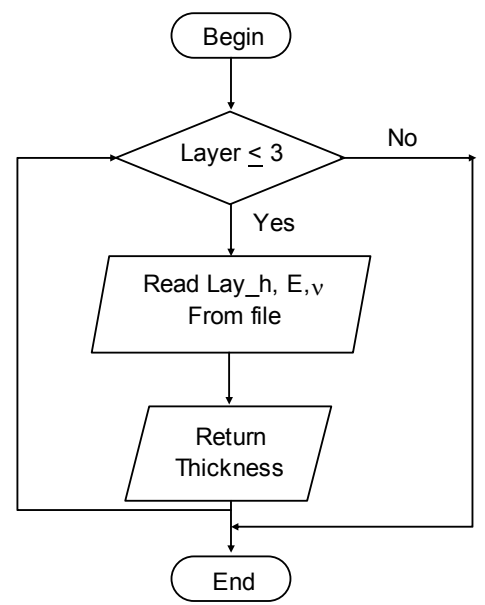

Appendix F - 2 Flow diagram (continiued) 


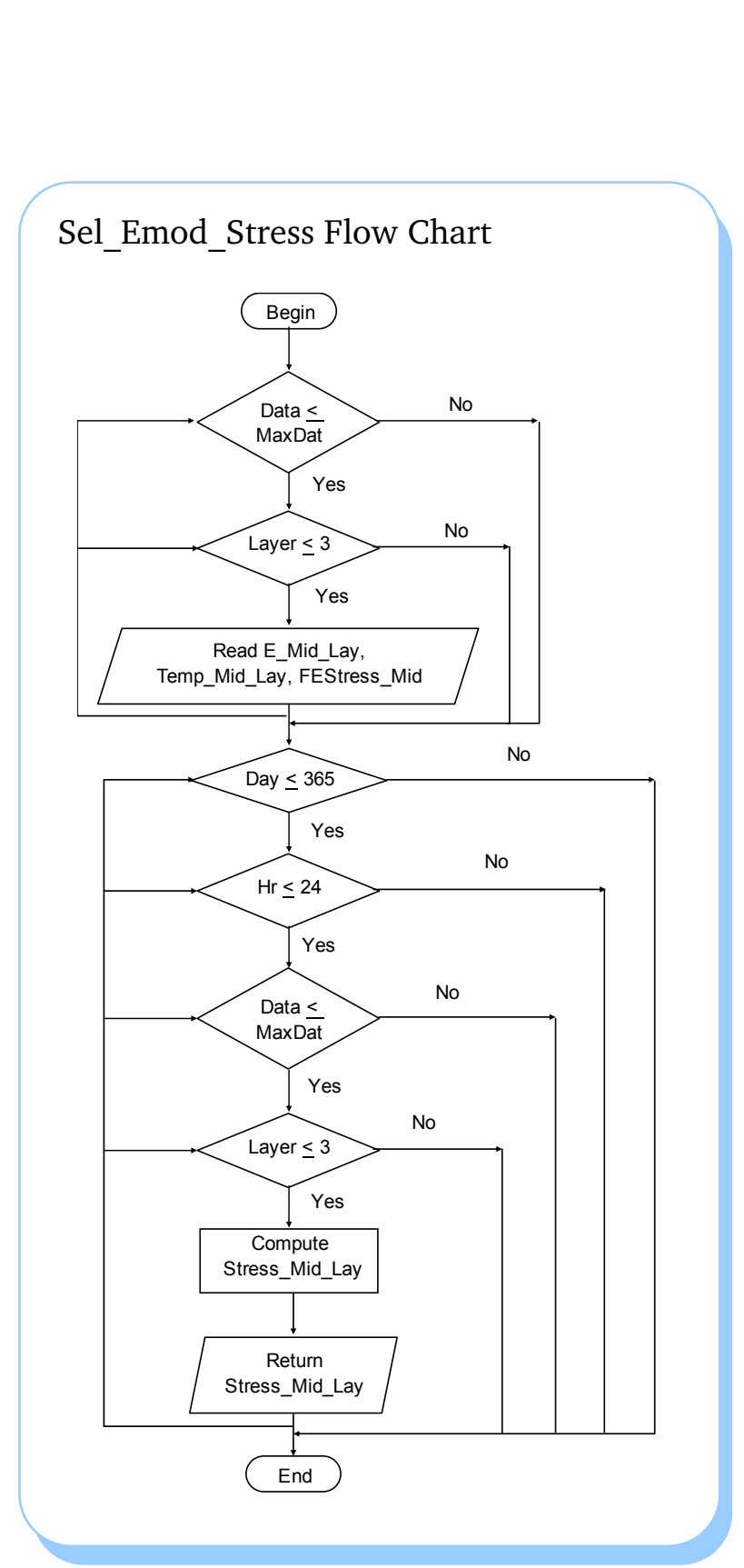

\section{Burgers Subroutine}

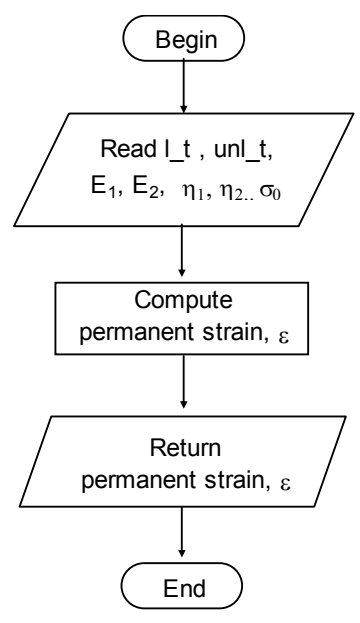

Deformation Flow Chart

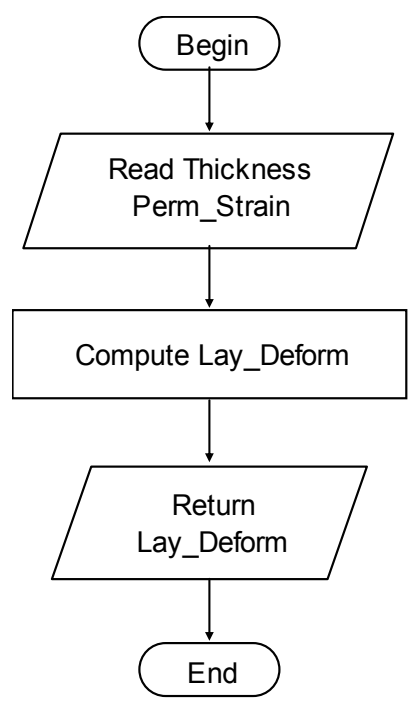

Appendix F - 3 Flow diagram (continiued) 